\title{
Quantifying the uncertainty caused by sampling, modeling, and field measurements in the estimation of AGB with information of the national forest inventory in Durango, Mexico
}

\author{
Dissertation \\ to attain the doctoral degree Doctor of philosophy (Ph. D.) \\ of the Faculty of Forest Sciences and Forest Ecology \\ Georg-August-Universität Göttingen
}

\author{
Submitted by \\ Ramón Trucíos Caciano \\ born on the $21^{\text {st }}$ March 1976 in Puebla, Mexico
}

Göttingen, May 2020 
First referee: Prof. Dr. Christoph Kleinn

Second referee: Prof. Dr. José Javier Corral Rivas

Date of oral examination: April 20th, 2020 


\section{Acknowledgement}

It is a great pleasure and honor for me to recognize my supervisor, Prof. Dr. Christoph Kleinn, for giving me the opportunity to do the $\mathrm{PhD}$. Thanks to his successful guide, and support through the feedback of my doubts and questions around my thesis work. I also appreciate his always good readiness to talk and his firm commitment to maintain an atmosphere of joy towards the thesis work. In the same way, I want to thank Dr. Lutz Fehrmann, Dr. Hans Fuchs and Dr. Cesar Perez Cruzado, who in their areas of expertise provided great ideas for the discussion of my research topic.

Financial support was very important during this period of study. I am grateful, therefore, with two important institutions, which contribute in the preparation of quality scientists in Mexico. The National Council of Science and Technology in Mexico (CONACYT in Spanish) assigned me the scholarship for maintenance and tuition fees, and the Secretary of Public Education in Mexico (SEP in Spanish) granted me a supplementary maintenance scholarship; as well as the German Academic Exchange Service (DAAD in German) for the support with the finishing grant.

My acknowledgment goes also to the National Institute of Research in Forestry, Agriculture and Livestock (INIFAP in Spanish), Mexico. Especially to the direction and administration of the National Center of Disciplinary Research in Relationship Water-Soil-Plant-Atmosphere (CENIDRASPA in Spanish), Dr. Juan Estrada Ávalos and Dr. José Antonio Cueto Wong, for their support through the scholarship and for give me the opportunity to make this great and important achievement in my professional and personal life.

I would like to acknowledge the support received from Prof. Dr. J. Javier Corral Rivas and Prof. Dr. Carlos Antonio López Sánchez from Juarez University of Durango State (UJED in Spanish) for accepting my collaboration and short stay to make field measurements in El Salto, Durango with the project "Installation of a trial of thinnings in forests of the UMAFOR 1008". In addition, I thank the collaboration in the fieldwork made by Roberto Flores and Daniel Molina Medrano. Likewise, I appreciate the cooperation and support of Prashant Paudel for having worked together for field measurements in Göttingen as part of the data analyzed in this thesis.

It was a pleasure to share this time with my colleagues at the Chair of Forest Inventory and Remote Sensing: Collins Kukunda, Dr. Damayanti Sarodja, Dr. Henning Aberle, Dr. Dengkui Mo, Dr. Dian Nuraini Melati, Dr. Xiaolu Tang, Dr. Almut Niebuhr, Kira Urban, Wanda Graf, Edwine Setia Purnama, Zihui Zhu, Adrian Straker, Sabine Schreiner, Dr. Philip Beckschäfer, Dr. Nils Nölke, Dr. Paul Magdon, Dr. Haijun Yang, Mrs. Silvia Wagner, Mr. Hendrik Heydecke, and Mr. Reinhard Schlote.

Great thanks to my family: Mariela "my love, support and inspiration", Juan Ramón e Ikal "my pride"; my parents Elvia Concepción and Juan Ramón†; my brothers Elvia and Ricardo and their families; the relatives of my wife; the entire relatives of Mexico; in Germany: my aunt Pita and her family, Ari "cuñada" and her family, Vale, Ernesto and his family, and of course the NERDS. Thanks to all of them for giving me their support, understanding and patience. 



\section{Summary}

Aboveground biomass (AGB) is related to the carbon content of the forest and forest carbon is a core variable for contemporary forest management and policy decisions. A credible and precise estimate of AGB is a prerequisite for the scientifically proper implementation of commitments made regarding the reduction of forest carbon emissions. With datasets of the Mexican National Forest Inventory (MNFI), this thesis estimates AGB in the temperate forests of Durango, a federal state of Mexico, evaluating the uncertainty of the estimate of total AGB and how different error sources contribute to the overall error. In addition to AGB, also the basal area was analyzed which is closely correlated to AGB but does not have the error source of coming from the application of allometric models.

The thesis covers empirical studies for various sources of errors, with a focus on measurement errors. Re-measurement experiments were conducted to quantify such measurement errors, applying mathematical-statistical techniques to model these errors. For the predictions of AGB, allometric models were selected according to the goodness-of-fit and the proximity of the study area of the fitted model. The plot design provided another source of uncertainty (uPlot) that was included in the analysis. The errors from the different steps in the AGB estimation process were propagated towards the total error by two techniques: (1) following the Guide to the Expression of Uncertainty in Measurement (GUM) and (2) by Monte Carlo simulation Method (MCM). In an additional study, AGB was regionalized for the study area using Landsat imagery as carrier data. To establish the corresponding remote sensing-based models, a set of vegetation indices and textures metrics were extracted from Landsat imagery and processed in a stepwise multiple regression analysis. Using the estimates of MNFI as response variables and Landsat imagery information as predictor variables, optimal models to estimate AGB were fitted. The regression models using texture metrics from Landsat imagery improve the AGB and the basal area estimates in the temperate forest of Durango, compared with regression models without texture metrics.

With respect to the tree measurement errors, they ranged from $0.133 \mathrm{~cm}$ to $1.197 \mathrm{~cm}$ for DBH (uDBH) and $0.348 \mathrm{~m}$ to $1.505 \mathrm{~m}$ for total height $\mathrm{TH}(\mathrm{uTH})$. uTH contributed most to the overall measurement error (uMes), accounting for $37 \%$ to $62 \%$ of the uMes. The uncertainty (error) induced by the allometric biomass model (UAM) was estimated by the mean square error (MSE) as the result of the model fit. The MSE of the uAM was combined with the uMes to estimate the treelevel uncertainty (uTree). In this step of error propagation, we estimated the two opposite results in two study cases. In a pine forest, uAM contributed $97.8 \%$ to the uTree; and in a broad-leaved forest, uMes contributed $98.9 \%$ to the uTree. Combining the uncertainties from tree level (uTree) to stand level, the non-sampling uncertainty or uNS $(\mathrm{uAM}+\mathrm{uDBH}+\mathrm{uTH})$ was estimated. The non-sampling uncertainty was combined with the standard error, estimated at sampling, and when propagating all sources of errors to the total uncertainty in AGB estimation, the analysis showed that the standard error contributed by far most to the total error with over $98 \%$ in the study cases of this thesis.

The ranking of the contributions of the different error sources to the propagated error of total AGB in Durango, Mexico was $\mathrm{SE}>\mathrm{uAM}>\mathrm{uTH} \geq \mathrm{uPlot} \geq \mathrm{uDBH}$ with values of $99.41 \%>0.53 \%>0.03 \% \geq 0.02 \% \geq 0.02 \%$, illustrating very clearly that in large-area forest monitoring, the sampling induced error is by far the most relevant, while measurement and model errors can 
almost be ignored - always. However, assuming that measurement and model errors are random errors and biases are absent.

Applying re-measurements was an efficient way to estimate and describe the measurement errors in $\mathrm{DBH}$ and TH. Through the application of the GUM Method, error propagation is decomposed into sources and processes, and it is better understood how uncertainties are combined. The Monte-Carlo simulation Method (MCM) also proves to be an effective, practical and reliable way to approximate the total AGB uncertainty estimate with acceptable ranges of probable error at scales of the MNFI. The results in the error propagation by the GUM Method and by the MCM are equivalent.

We found that TH measurement errors have a greater contribution than DBH measurement errors at the tree-level. Furthermore, when the main contribution to the uncertainty at tree-level comes from uAM instead of uMes, then the total estimate of uNS at stand-level is proportional to the number of the trees. However, a ratio of uMes>uAM produces a total uNS estimate at stand level that is proportional to the contribution according to the size of the tree. Therefore, in this last relation, a greater contribution to the total NS estimate is made by the trees with the largest AGB estimated. 


\section{Zusammenfassung}

Die oberirdische Biomasse (Above Ground Biomass = AGB) is eine wesentliche Variable für die Schätzung von Kohlenstoffvorräten im Wald. Die Quantifizierung des Kohlenstoffvorrats ist in Zeiten des Klimawandels eine zentrale Größe für die Waldbewirtschaftung und für entsprechende politische Entscheidungen. Eine wissenschaftlich präzise und damit glaubwürdige Schätzung der oberirdischen Biomasse ist eine der wesentlichen Voraussetzungen im Zusammenhang mit der korrekten Umsetzung der Verpflichtungen, die die Vertrasgparteien zur Klimakonvenion UN-FCCC eingegangen sind, um die Kohlenstoffemissionen aus Wald zu reduzieren.

Die vorliegende Dissertationsschrift wertet Datensätze der mexikanischen nationalen Waldinventur (MNWI) aus dem mexikanischen Bundesstaat Durango aus. Sie analysiert die verschiedenen Fehlerquellen (Residualvariabilität) bei der Schätzung der oberirdischen Waldbiomasse und bewertet, wie sich diese Fehlerquellen im Rahmen der Fehlerfortpflanzung auf den Gesamtfehler auswirken. Hintergrund dieser Analysen ist, dass eine effiziente Verfahrensverbesserung insbesondere an den Teilschritten des Schätzprozesses stattfindet, an denen ein gegebener Ressourceneinsatz die höchstmögliche Reduktion des Gesamtfehlers bewirkt. Ergänzend zur oberirdischen Biomasse erfolgten entsprechende Analysen auch für die Bestandesgrundfläche, die eng mit der oberirdischen Biomasse korreliert ist, bei der aber die Modellfehler aus allometrischen Biomassenmodellen nicht zum Tragen kommen.

Die Dissertationsschrift umfasst vier empirische Teilstudien zu den verschiedenen Fehlerquellen, wobei ein Schwerpunkt auf Messfehlern liegt. Zur Quantifizierung solcher Messfehler erfolgten Wiederholungsmessungen, wobei mathematisch-statistische Techniken zur Modellierung dieser Fehler zum Einsatz kamen. Die Vorhersagen der oberirdischen Biomasse erfolgten mittels allometrischer Modelle, die nach der Anpassungsgüte und der Nähe zum Untersuchungsgebiet ausgewählt wurden. Das Plot-Design stellte eine weitere Unsicherheitsquelle (uPlot) dar und wurde als solche in die Analyse einbezogen. Die Fehler, die sich aus den verschiedenen Schritten des oberirdischen Biomasse-Schätzprozesses ergaben, wurden mit zwei Techniken der Fehlerfortpflanzung auf den Gesamtfehler hochgerechnet: (1) gemäß des ISO-Leitfadens des Zuverlässigkeitsmanagements (GUM auf Englisch) und (2) durch die Monte-CarloSimulationsmethode (MCM). Eine weitere empirische Studie hatte die Regionalisierung der oberirdischen Biomasse für das Untersuchungsgebiet zum Gegenstand, wobei Landsat-Bilder als Trägerdaten verwendet wurden. Um die entsprechenden fernerkundungsbasierten Modelle zu erstellen, wurde eine Set von Vegetationsindizes und Texturmetriken aus den Landsat-Bildern abgeleitet und in einer schrittweisen multiplen Regressionsanalyse verarbeitet. Unter Verwendung der Plot-Daten aus der nationalen Waldinventur von Mexiko als Response-Variablen und LandsatBildinformationen als Prädiktor-Variablen, wurden Modelle zur Schätzung der oberirdischen Biomasse optimiert. Texturmetriken als Prädiktorvariablen verbessern die AGB- und die Grundflächenschätzungen in der Studie in Durango im Vergleich zu Regressionsmodellen ohne Texturmetriken.

Die Messfehler der Bäume reichten von 0,13 cm bis 1,207 cm für den BHD (uBHD) und von 0,348 $\mathrm{m}$ bis 1,505 $\mathrm{m}$ für die Gesamthöhe $\mathrm{GH}(\mathrm{uGH})$. uGH trug am meisten zum Gesamtmessfehler (uMes) bei und erreichte $37 \%$ bis $62 \%$ der messfehlerbedingten Ungenauigkeit uMes. Die durch das allometrische Biomasse-Modell (uAM) induzierte Unsicherheit (Fehler) wurde durch den mittleren 
quadratischen Fehler (MSE) als Ergebnis der Modellanpassung abgeschätzt. Der Modellfehler wurde mit dem Messfehler uMes kombiniert, um die Unsicherheit pro Baum (uBaum) zu bestimmen. Die Beiträge der beiden Fehlerquellen uAM (Modellfehler) und uMes (Messfehler) zum Gesamtfehler der baumweisen Biomasseschätzung (uBaum) wurden in Durango in einem Kiefernwald und einem Laubwald exemplarisch bestimmt und waren sehr unterschiedlich: im Kiefernwald trug uAM 97,8\% zu uBaum bei; und in einem Laubwald trug uMes 98,9\% zu uBaum bei. Im nächsten Schritt erfolgte eine Hochrechnung der Fehler pro Baum auf das Gesamtgebiet. Die Hochrechnung der Biomasse erfolgt aufgrund von Stichprobenverfahren, so dass hier der Standardfehler als weitere Fehlerquelle zu Modell- und Messfehler hinzukommt. Bei der Fortpflanzung aller Fehlerquellen auf die Gesamt-Unsicherheit der oberirdischen BiomasseSchätzung zeigte die Analyse, dass der Standardfehler (SE) mit über $98 \%$ bei weitem am meisten zum Gesamtfehler beitrug; diese überragende Bedeutung des Stichprobenfehlers bei der Biomasseschätzung aus Waldinventuren ist konform mit anderen Studien, insbesondere mit großräumigen Waldinventuren mit großen Stichprobenumfängen.

Die Rangfolge der Beiträge der verschiedenen Fehlerquellen zm Gesamtfehler der Schätzung der oberirdischen Biomasse war SE $>\mathrm{uAM}>\mathrm{uGH} \geq \mathrm{uPlot} \geq \mathrm{uDBH}$ mit Werten von $99,41 \%>0,53 \%>$ $0,03 \% \geq 0,02 \% \geq 0,02 \%$. Dieser Befund illustriert sehr deutlich, dass im großflächigen Waldmonitoring der Stichprobenfehler mit Abstand am relevantesten ist, während Mess- und Modellfehler fast immer vernachlässigbar klein sind; diese Aussage gilt unter der Annahme, dass Mess- und Modellfehler zufällige und keine systematischen (Bias) Fehler sind.

Die hier durchgeführten Wiederholungsmessungen stellen einen effizienten Weg dar, die Messfehler in BHD und GH zu quantifizieren.

Beide Ansätze zur Fehlerfortpflanzung führten zu vergleichbaren Ergebnissen. Die Anwendung der analytischen Methode der Fehlerfortpflanzung (GUM-Methode) erfordert eine Zerlegung in Quellen und Prozesse und erzeugt somit ein besseres Verständnis für die Kombination der Fehlerquellen. Die Monte-Carlo-Simulation (MCM) erweist sich auch als effektive, praktische und zuverlässige Methode, um die gesamte Unsicherheitsschätzung der oberirdischen Biomasse mit akzeptablen Bereichen wahrscheinlicher Fehler auf Skalen der MNWI zu approximieren.

Es zeigte sich, dass auf Ebene der Biomasseschätzung für einzelne Bäume die Messfehler in GH einen größeren Beitrag leisten als die Messfehler in BHD. Wenn der Hauptbeitrag zur Unsicherheit der Schätzung für einen einzelnen Baum von uAM statt von uMes stammt, dann ist die gesamte uNS-Schätzung auf Bestandesebene proportional zur Anzahl der Bäume. Eine Beziehung uMes>uAM erzeugt jedoch eine gesamte uNS-Schätzung auf Bestandesebene proportional zur Baumgröße. Daher wird in dieser letzten Beziehung ein größerer Beitrag zur gesamten NSSchätzung von den Bäumen mit der größten geschätzten oberirdischen Biomasse geleistet. 
Table of Content

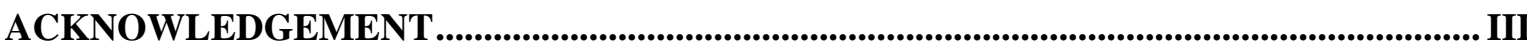

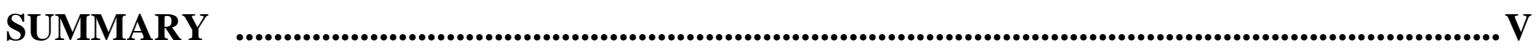

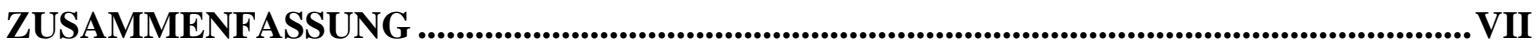

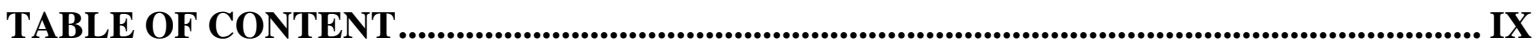

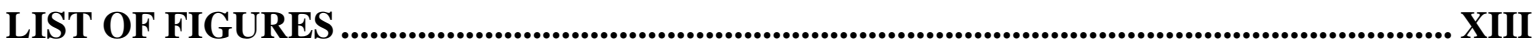

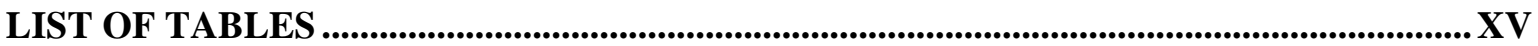

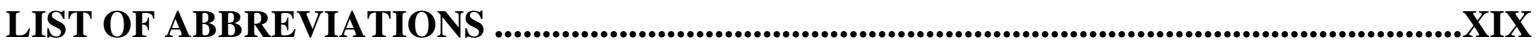

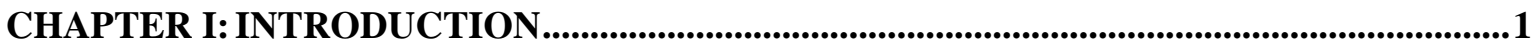

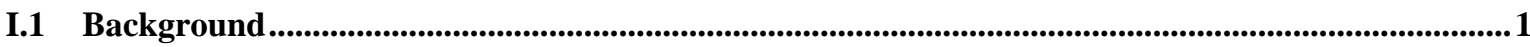

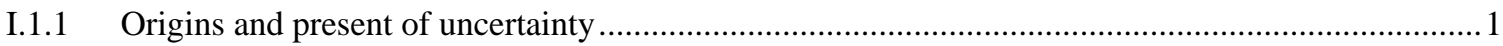

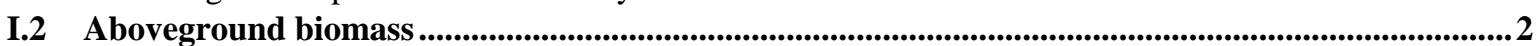

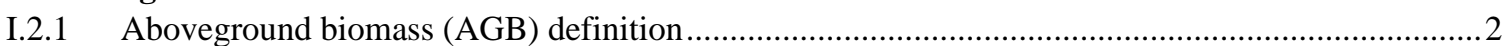

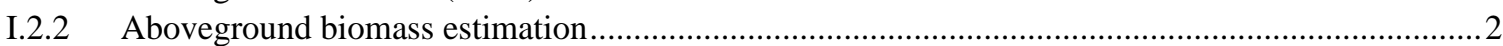

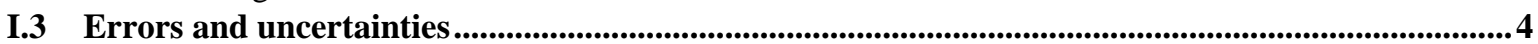

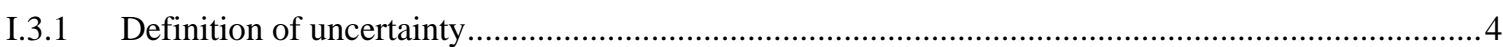

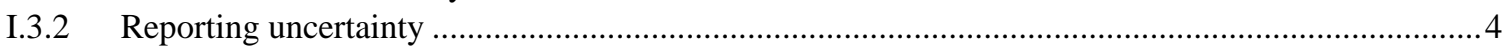

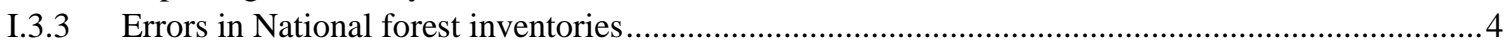

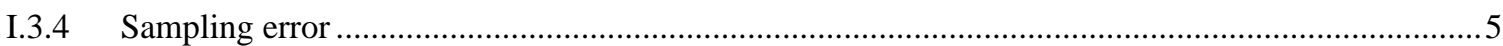

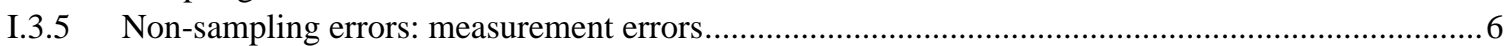

I.3.6 Non-sampling errors: prediction errors due to allometric models .................................................... 6

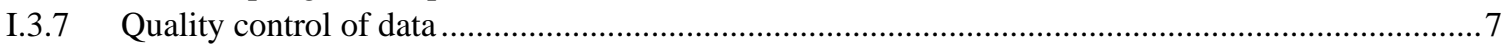

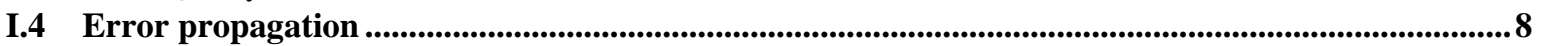

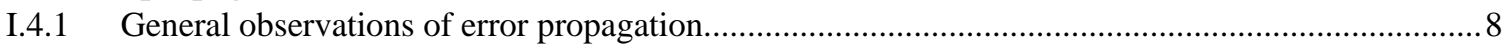

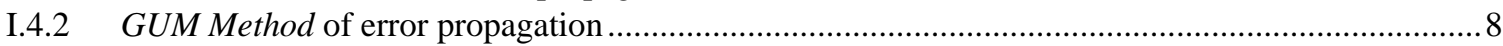

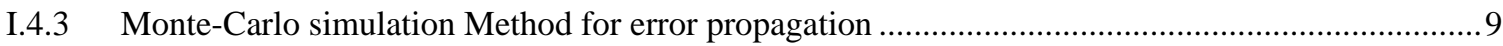

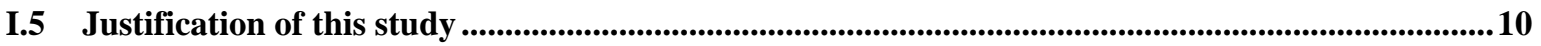

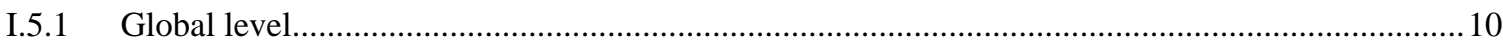

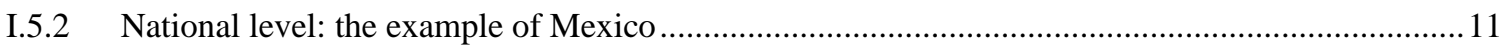

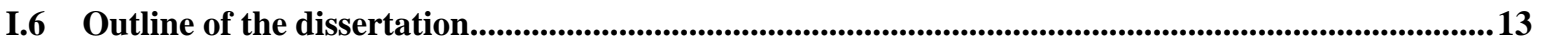

I.7 Objective and research questions....................................................................................................................14

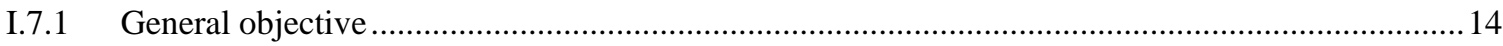

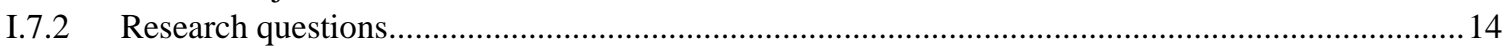

CHAPTER II: MATERIALS ....................................................................................................... 17

II.1 Study Area

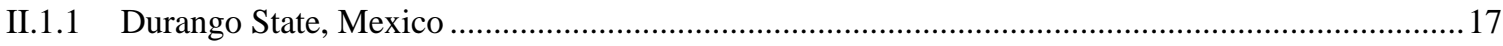

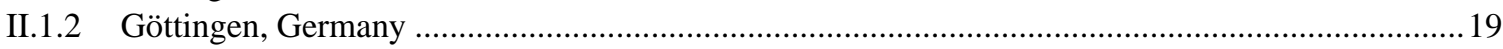

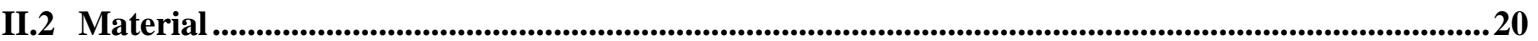

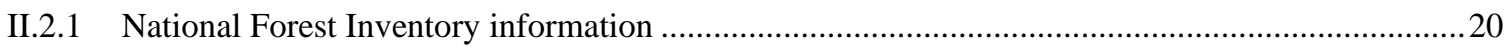

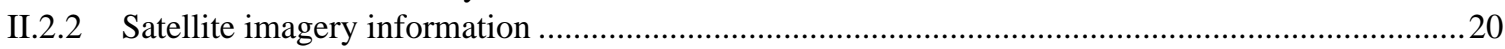

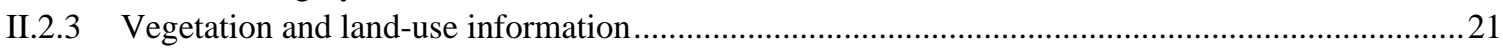

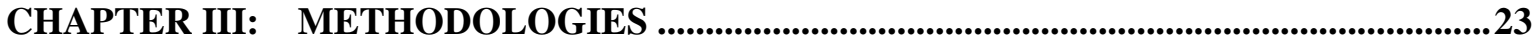


III.1 Empirical study 1: Double measurement of dasometric variables to estimate the measurement uncertainty and error propagation in aboveground biomass estimation in pine forests........................... 23

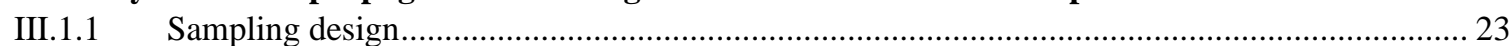

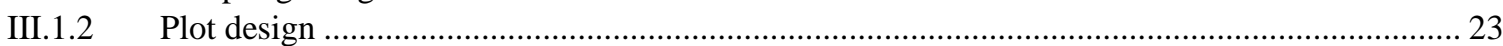

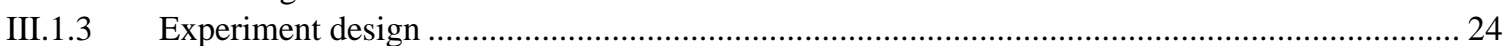

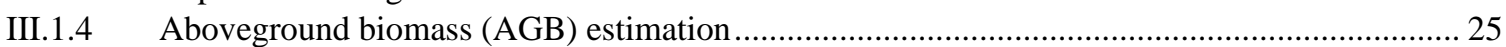

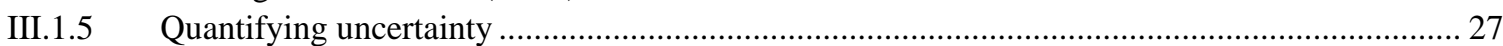

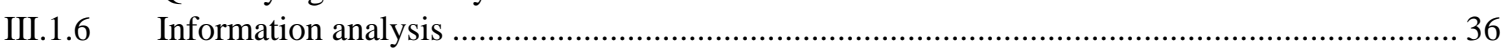

III.2 Empirical study 2: Comparison of aboveground biomass estimates from two types of dasometric measuring equipment for the variables $\mathrm{DBH}$ and $\mathrm{TH}$, a case study developed in beech forest near

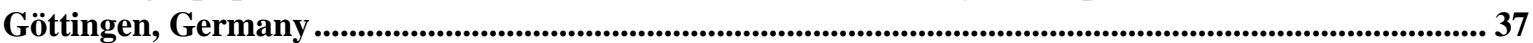

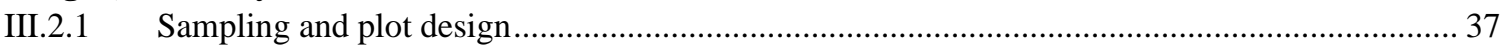

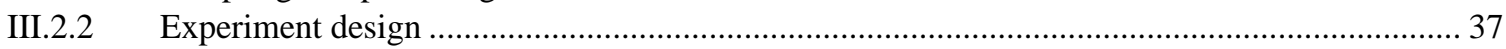

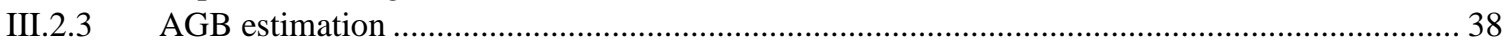

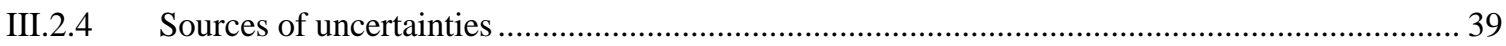

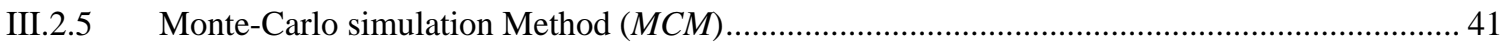

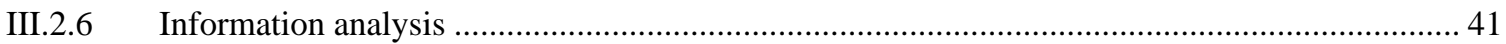

III.3 Empirical study 3. Propagation of errors in the AGB estimation for the state of Durango, Mexico,

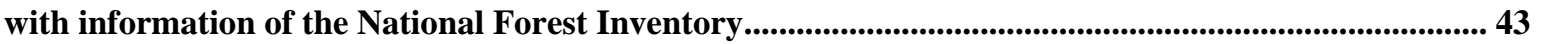

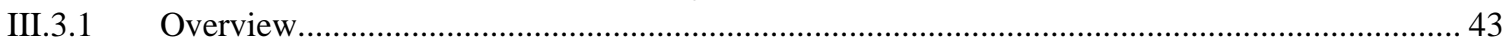

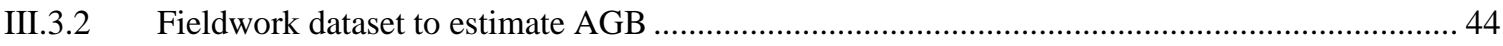

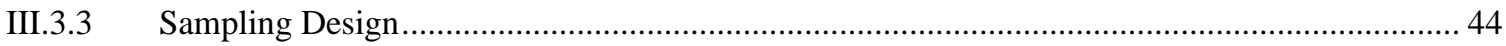

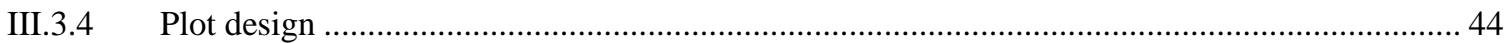

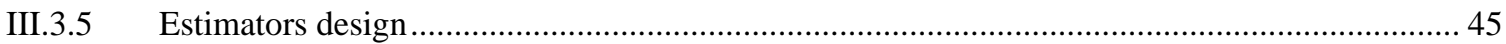

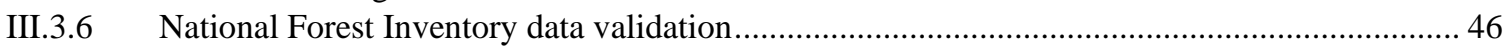

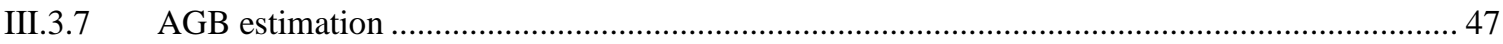

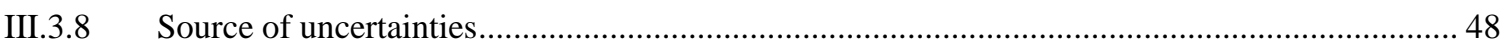

III.3.9 Error propagation with Monte-Carlo simulation Method $(M C M)$.............................................. 49

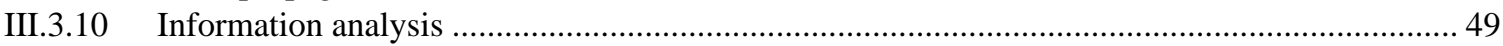

III.4 Empirical study 4: Modelling and mapping AGB for the state of Durango ........................................ 51

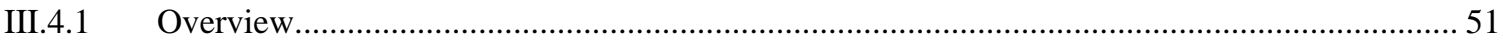

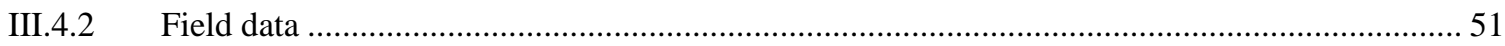

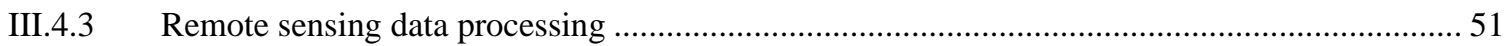

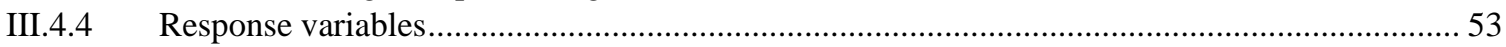

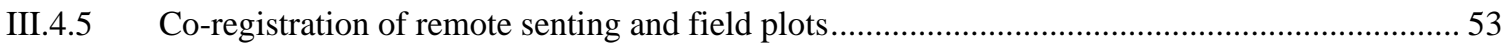

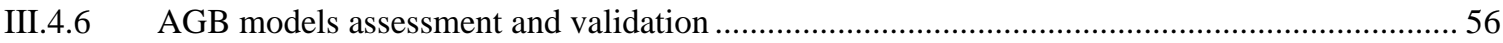

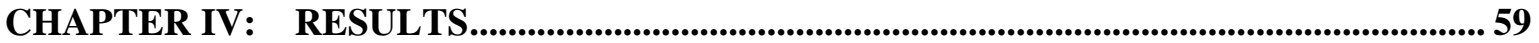

IV.1 Empirical study 1: Double measurement of dasometric variables to estimate the measurement uncertainty and error propagation of aboveground biomass estimation in pine forests...........................59

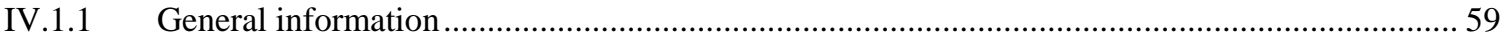

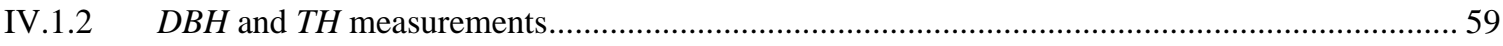

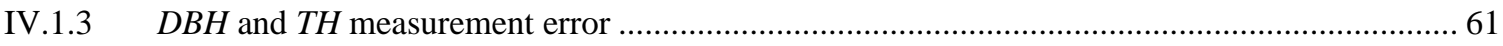

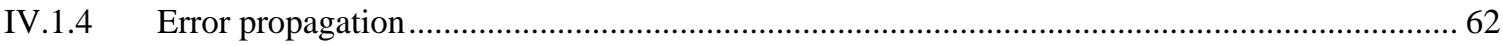

IV.2 Empirical study 2: Comparison of aboveground biomass estimates from two types of dasometric measuring equipment for the variables $\mathrm{DBH}$ and $\mathrm{TH}$, a case study developed in beech forest near

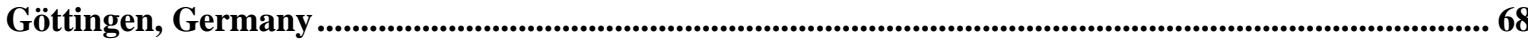

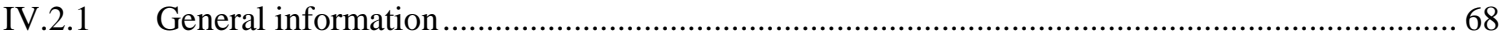

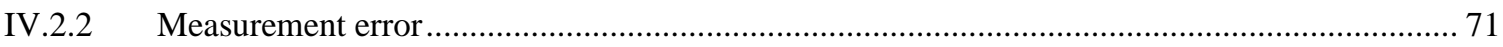

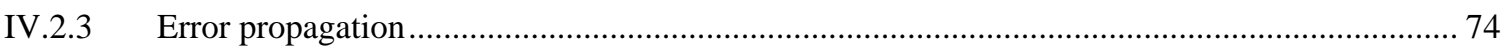

IV.3 Empirical study 3: Propagation of errors in the AGB estimation for the state of Durango, Mexico, with information from the National Forest Inventory......................................................................................... 83

IV.3.1 General information on the Mexican National Forest Inventory dataset .................................. 83 


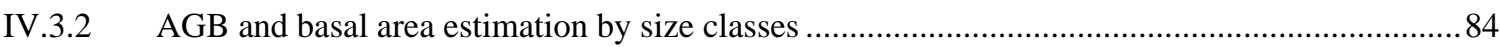

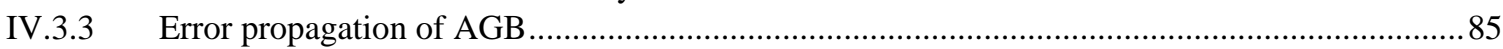

IV.4 Empirical study 4: Modelling and mapping AGB for the state of Durango.....................................92

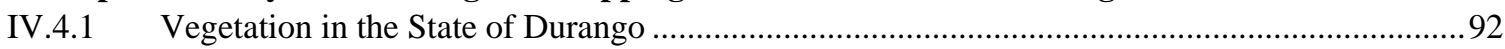

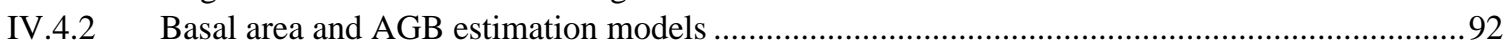

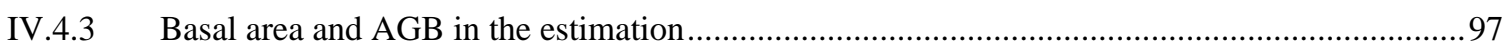

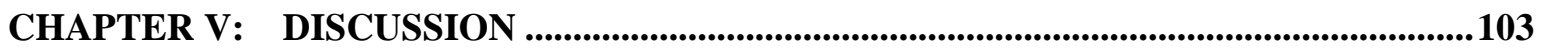

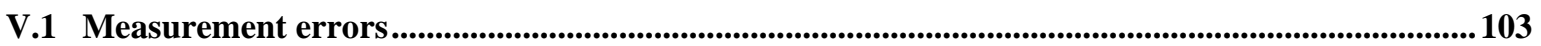

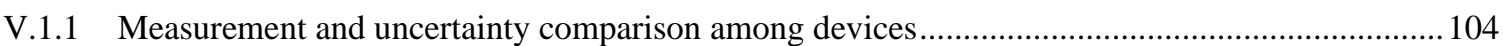

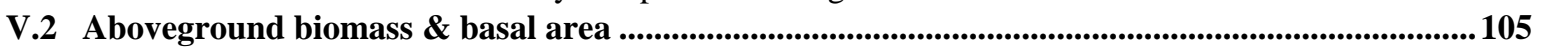

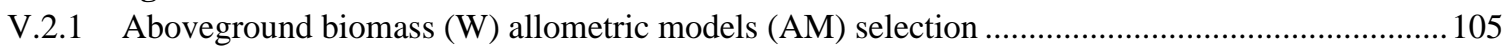

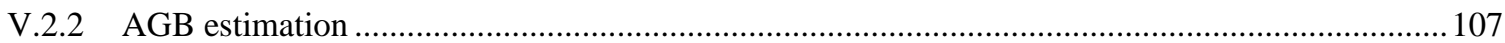

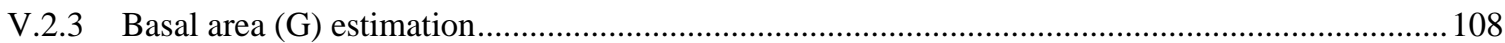

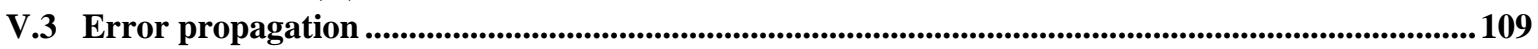

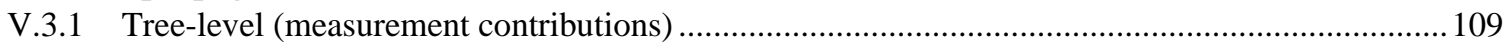

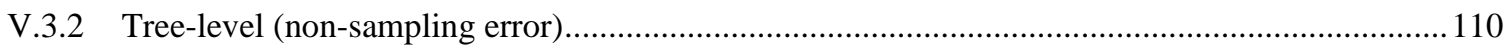

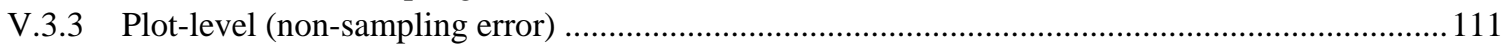

V.3.4 Stand-level GUM Method (non-sampling \& sampling errors)....................................................111

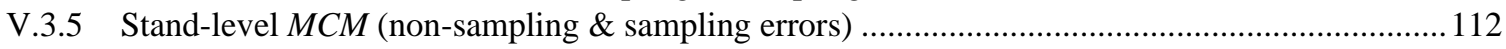

V.4 AGB and basal area estimation with Landsat imagery information ..............................................115

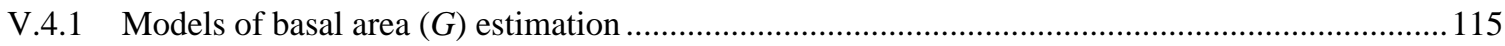

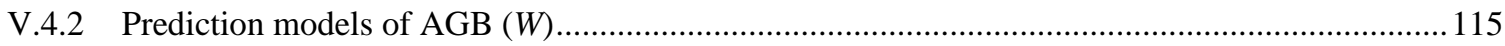

V.4.3 Basal area $(G)$ and AGB $(W)$ estimation in strata of temperate forest .......................................... 116

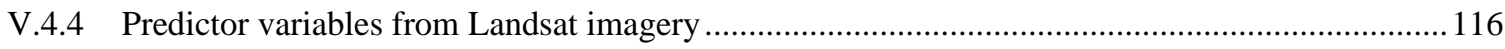

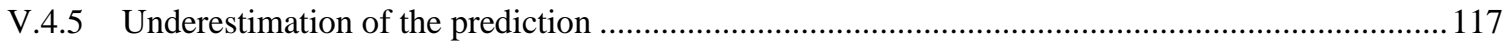

V.4.6 Model-based comparison with sampling-based estimations ..........................................................118

CHAPTER VI: CONCLUSIONS .................................................................................................. 121

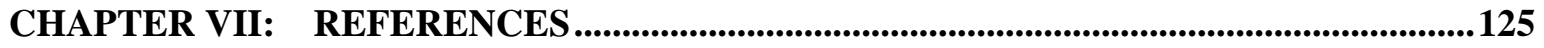

CHAPTER VIII: APPENDIX..................................................................................................137

VIII.1 Appendix I .................................................................................................................................................137

VIII.2 Appendix II .......................................................................................................................................140

VIII.3 Appendix III ........................................................................................................................................141

VIII.4 Appendix IV ........................................................................................................................................143

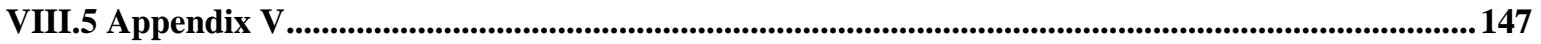

VIII.6 Appendix VI ............................................................................................................................................................. 156

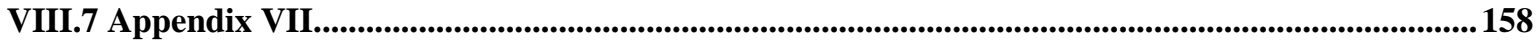

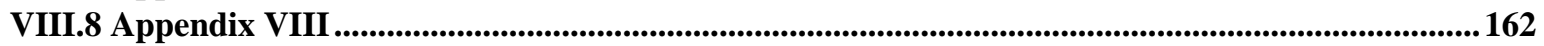





\section{List of Figures}

Figure I-1. Levels of uncertainty (source: Marchau et al., 2019) ............................................................. 1

Figure I-2. Direct and indirect methods of AGB estimation............................................................... 3

Figure I-3. Total error in the measurement as a product of systematic errors and random errors. Modified

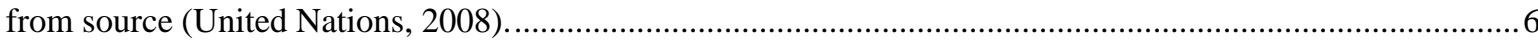

Figure I-4. Sources of uncertainty evaluated in the error propagation of AGB using NFI fieldwork data........ 8 Figure I-5. Scenarios of carbon emission from land-use change in the tropical forests. Source: Houghton, 2005 modified by Quegan et al., 2012.

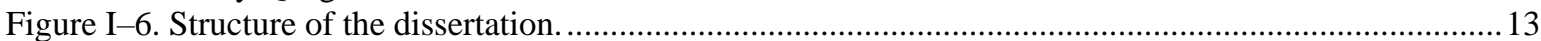

Figure II-1. Durango State in the context of Mexico.................................................................. 17

Figure II-2. Location map of plot sites and UMAFOR 1008 in the State of Durango. ............................... 18

Figure II-3. Location map of the study area in the Göttingen Forest, Germany ........................................19

Figure II-4. Left: Grid of Primary sampling units (PSU) of the MNFI for the temperate forest in Durango.

Right: Landsat 5 mosaic imagery false-color composite with RGB: 432 combination to enhance vegetation in red color, covering all forested lands in Durango. ........................................................................20 Figure II-5. Vegetation and land-use limits of Durango for the LUVS of 2007-2008 (Serie IV) and 2012-2013 (Serie V).

Figure II-6. Vegetation strata limits of temperate forest in Durango according to vegetation Series IV and V.

Figure III-1. Pictures of the pine forest in El Salto, Durango, where the sampling sites were located

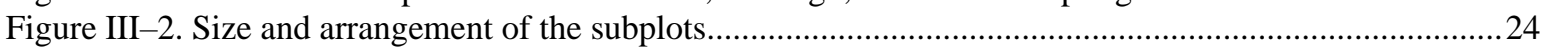

Figure III-3. Flow chart to select the allometric model for AGB estimation, adapted from CONAFOR

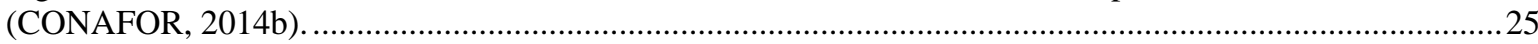

Figure III-4. Discrepancies dispersion across the size of the tree and distribution of the discrepancies for the

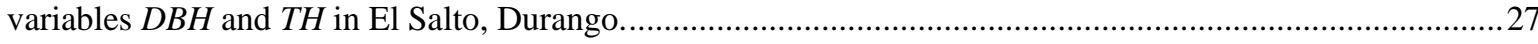

Figure III-5. Linear regression relating discrepancies in measurement with the size of variable measured

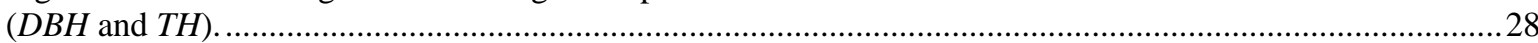
Figure III-6. Probability Density Function of uncertainties for $D B H$ and $T H$ measurements in the study area.

Figure III-7. Flowchart to estimate AGB error propagation adapted from two publications, the Evaluation of measurement by JCGM (2010) and the IPCC Guidelines for National GHG Inventories (2006).................33 Figure III-8. Iterations made to select the optimal number of iterations for $M C M$ in the uncertainty estimation of AGB with information of El Salto, Durango. Figure III-9. Flowchart for error propagation of measurement and model uncertainties in El Salto, Durango,

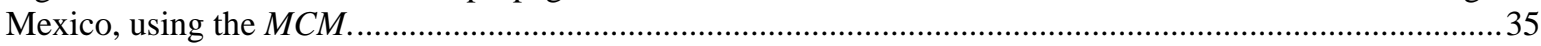
Figure III-10. The pictures give an idea of the study area and the systematic sampling design is given. ........ 37 Figure III-11. Essay of iterations to select the optimal number of iterations for $M C M$ in the uncertainty estimation of AGB with information of Göttingen.

Figure III-12. Durango coverage grid for the tree main vegetation types. ................................................45 Figure III-13. Form and distribution of PSU and SSU in the MNFI in Mexico. Source (CONAFOR, 2012c).45 Figure III-14. Flow diagram for database debugging implemented to select the information to analyze from the database of MNFI in Durango.

Figure III-15. Tree distribution in the relationship $D B H(\mathrm{~cm}) \& T H(\mathrm{~m})$ for El Salto $2015(\mathrm{n}=4262)$, MNFI

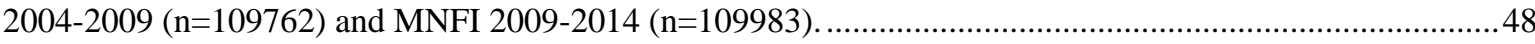
Figure III-16. Flowchart for error propagation of measurement, model and plot design uncertainties for MNFI

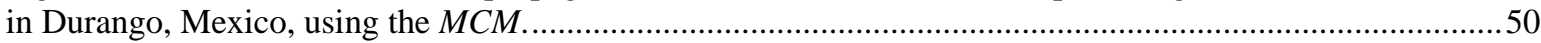
Figure III-17. AGB estimation and mappaing flowchart.....................................................................51 Figure III-18. Texture metrics construction with the image (spectral band or vegetation index), window size (in pixels) and the Haralik texture estimate.

Figure IV-1. Basal area and AGB estimation of the study area. Above, estimate by $D B H$ class every $5 \mathrm{~cm}$; below, estimate by $T H$ class every $2.5 \mathrm{~m}$. Figure IV-2. Left: $D B H$ data boxplot, histograms of Measurement (MI) and Re-measurement (M2). Right: a similar case for $T H$. Bottom, the trend line of $M 2$ versus $M 1$ for $D B H$ and $T H$. Figure IV-3. Probability Density Functions (PDF) fitted with $D B H$ measurement errors according to $D B H$ class. 
Figure IV-5. Basal area and AGB estimation. Above, estimate by $D B H$ class every $20 \mathrm{~cm}$; below, estimate by

$T H$ class every $10 \mathrm{~m}$. The bars represent the frequency of threes per size class.

Figure IV-6. Relationship between $\mathrm{DBH}$ and $\mathrm{TH}$ for mean values of the 250 trees in re-measurement plots made with Caliper and Blume-Leiss $(C B)$ and with Tape and Vertex (TV).

Figure IV-7. Left-top: $D B H$ boxplot and overlapped histograms of Caliper $(C)$ and Tape $(T)$ measurements.

Right-top, graphs of $T H$ made with Blume-Leiss $(B L)$ and Vertex $(V)$. Bottom: comparison of measurements made with two devices

Figure IV-8. Probability Density Function (PDF) fitted with $D B H$ measurement error according to $D B H$ class, for Caliper and Tape measurement. a) and b) are PDF fitted for the whole dataset of Caliper and Tape, respectively.

Figure IV-9. Probability Density Function fitted with $T H$ measurement error according to $T H$ class, for Blume-Leiss and Vertex measurements. a) and b) are PDF fitted for the whole dataset of Blume-Leiss and Vertex $I V$, respectively.

Figure IV-10. Contribution of uncertainties from $\mathrm{DBH}$ and $\mathrm{TH}$ measurements to the total non-sampling uncertainty of AGB estimate.

Figure IV-11. Comparison of uncertainty calculated per plot and measurement device, for three scenarios of measurement error in AGB estimation.

Figure IV-12. Basal area $(G)$ and AGB $(W)$ estimation for 2004-2009 MNFI in Durango, Mexico. Above, estimations made by $\mathrm{DBH}$ class; below, estimations made by $\mathrm{TH}$ class.

Figure IV-13. Basal area in the temperate forest of Durango, Mexico, with information from the MNFI and Landsat imagery.

Figure IV-14. AGB in the temperate forest of Durango, Mexico, with information from the MNFI and Landsat imagery.

Figure IV-15. AGB storage and uncertainty in AGB storage using information from two methods of estimation in the temperate forests of Durango, Mexico.

Figure VIII-1. Model fitted to estimate the measurement uncertainty, according to the tree size for Caliper $(D B H)$, Tape $(D B H)$, Blume-Leiss $(T H)$ and Vertex $I V(T H)$, with information of 250 trees in the study area of Göttingen.

Figure VIII-2. Comparison of uncertainty estimation (paired plot) per plot and per measuring device for each scenario used to estimate measurement error parameters. ............................................................. 155 Figure VIII-3. Basal area $(G)$ and AGB $(W)$ estimation for MNFI 2009-2014 in Durango, Mexico. Above, estimations made by $\mathrm{DBH}$ class; below, estimations made by $\mathrm{TH}$ class.

Figure VIII-4. Predicted vs. observed in AGB estimate $(G)$ when applying Landsat-adjusted models and MNFI information in Durango, Mexico.

Figure VIII-5. Residual vs. predicted graphs in basal area estimation $(G)$ applying linear regression models with Landsat and MNFI data.

Figure VIII-6. Predicted vs. observed in AGB estimate $(W)$ when applying Landsat-adjusted models and MNFI information in Durango, Mexico.

Figure VIII-7. Residual vs. predicted graphs in AGB estimation $(W)$ applying linear regression models with Landsat and MNFI data. 


\section{List of Tables}

Table I-1. Instruments and policies to implement actions for adaptation and mitigation of climate change in

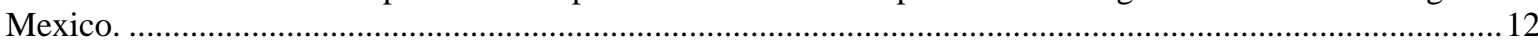

Table II-1. General information in sampling units of MNFI in Durango, Mexico....................................20

Table II-2. Satellite imagery scenes of Landsat used in this study to estimate AGB in Durango, Mexico.

$R M S E$ was expressed in meters and characterizes the result of the geometric correction with ground control

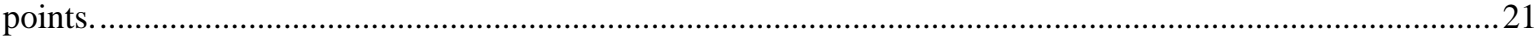

Table III-1. Allometric models used in the pine forest of El Salto, Durango to estimate AGB based in $D B H$

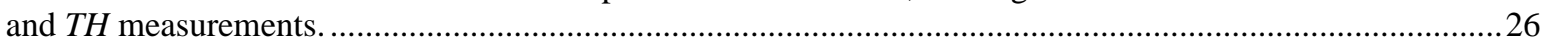

Table III-2. Methods and scenarios applied in the error propagation of AGB estimation in El Salto, Durango,

Mexico. .....

....36

Table III-3. Allometric AGB models used in the study area of Göttingen, Germany (Source: Fehrmann, 2006).

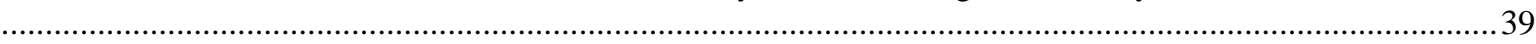

Table III-4. Goodness-of-fit for the models used in the study area of Göttingen using $D B H$ and $T H$

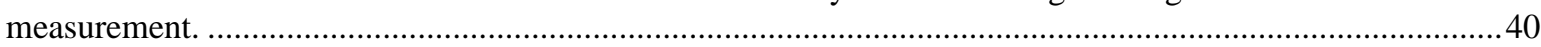

Table III-5. Reference datasets in AGB estimation for re-mesurement plots. ...........................................42

Table III-6. Scenarios of error propagation with GUM Method ...............................................................43

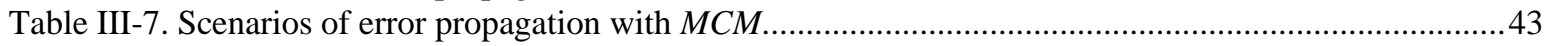

Table III-8. Sources of error contributing to error propagation scenario. ...............................................43

Table III-9. Aggregation levels used to classify vegetation with the number of clusters recorded in the MNFI

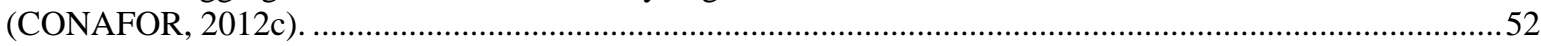

Table III-10. Vegetation indexes evaluated in this study based on spectral bands information of Landsat

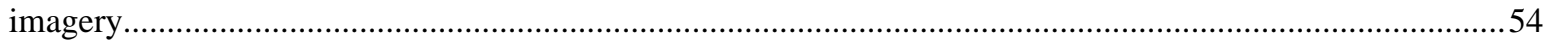

Table III-11. Texture variables used. $P(i, j)$ is the normalized co-occurrence matrix such that $\operatorname{sum}(i, j=0, N$ -

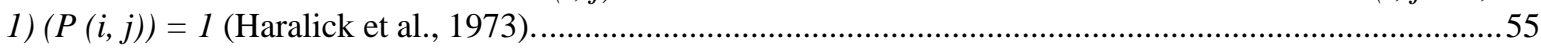

Table III-12. Vegetation indexes with the highest Pearson correlation coefficient, selected to apply on them

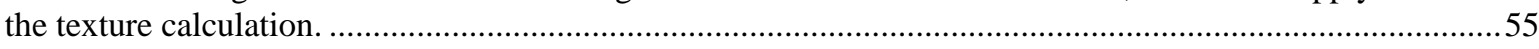

Table III-13. Landsat image predictor variables $(P V)$ used to evaluate models for estimating AGB and basal

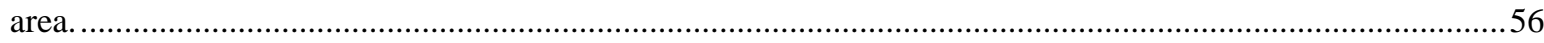

Table IV-1. Range and mean values of the measured and estimated variables in the pine forests of El Salto,

Durango.

Table IV-2. Uncertainty of the AGB calculation using three scenarios estimating measurement uncertainty

NDn, NDnC and RMSD.

Table IV-3. The total uncertainty of the AGB calculation from non-sampling uncertainty estimation sources.

Table IV-4. Uncertainty of the AGB calculation with $M C M$ using four uncertainty estimation scenarios along

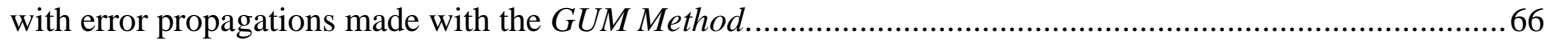

Table IV-5. Uncertainty of the AGB calculation with $M C M$ for non-sampling uncertainty sources and their

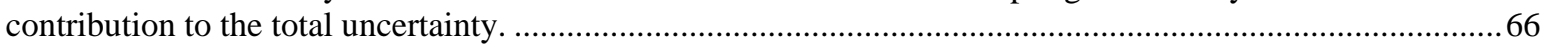

Table IV-6. Range of values for measurements and estimated variables in the study area............................68

Table IV-7. Statistics of measurements and estimated variables in the study area. ......................................68

Table IV-8. Uncertainty parameters for scenarios to estimate measurement error of $D B H$ and $T H \ldots \ldots \ldots \ldots \ldots .72$ Table IV-9. Uncertainty of the AGB calculation using four scenarios to estimate the measurement uncertainty

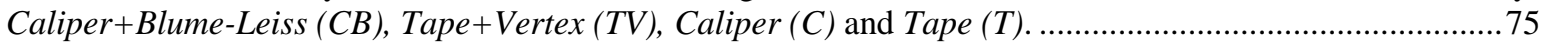

Table IV-10. Sources of uncertainty contribution in the AGB calculation for different scenarios of

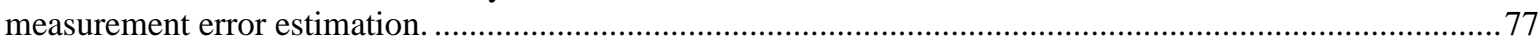

Table IV-11. Error propagation in AGB estimation with $M C M$ different scenarios to calculate measurement errors.

Table IV-12. Estimates of basal area $(G)$ and AGB $(W)$ calculated from the MNFI in the temperate forest of

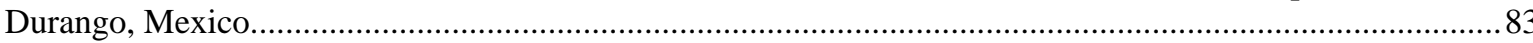

Table IV-13. Error propagation in AGB estimation using three scenarios of non-sampling uncertainty in

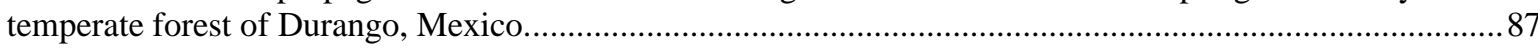

Table IV-14. Error propagation in AGB estimation, using three scenarios of non-sampling uncertainty in

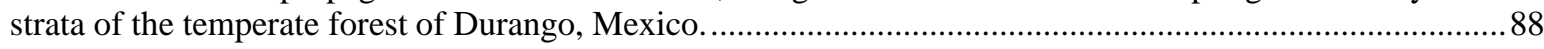
Table IV-15. Reference values at the substratum level with the sampling error as the source of uncertainty in AGB estimation of the temperate forests in Durango, Mexico. 
Table IV-16. Error propagation in AGB estimation, using three scenarios ( $B D, B D C$, Exp) of non-sampling uncertainty estimation in the study area.

Table IV-17. Error propagation by uncertainty source in AGB estimation, using three scenarios of nonsampling uncertainty in the temperate forests of Durango, Mexico. ................................................ 90 Table IV-18. Surface area by vegetation type and land-use for two time-series in the state of Durango,

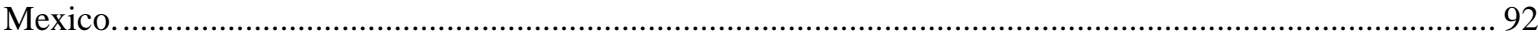

Table IV-19. Predictor variables $(P V)$ selected to estimate the response variables basal area $(G)$ and AGB $(W)$

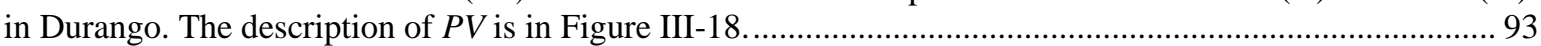
Table IV-20. Adjusted models for the basal area $(G)$ and AGB $(W)$, estimated from the 2004-2009 MNFI... 94 Table IV-21. Adjusted models for the basal area $(G)$ and AGB $(W)$, estimated with the 2009-2014 MNFI data.

Table IV-22. Bias estimate and goodness-of-fit of the adjusted models for the basal area $(G)$ and AGB $(W)$

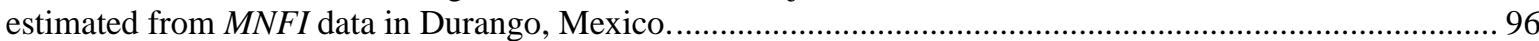

Table IV-23. Basal area $(G)$ and AGB $(W)$, estimate for temperate forest in Durango, Mexico.................... 97 Table IV-24. Estimates of basal area $(G)$ and AGB $(W)$ using methods based on forest inventory sampling and regression models, applied in the temperate forests of Durango, Mexico. 100

Table VIII-1. AGB allometric models $(A M)$ used in $M N F I$ in the state of Durango with the number of trees registered. $A M$ data: $\mathrm{n}=$ number of sampled trees to fit the $A M ; R^{2}=$ coefficient of determination; $R M S E=$ rootmean-square error; $W=\mathrm{AGB}$ in kg per tree; Variables used to fit the $A M: D B H, T H$, basal area $(G)$, crown volume $(\mathrm{CVol})$; $\mathrm{ND}$ (= no data) is given when data were not reported; $\mathrm{BR}=\mathrm{Brazil}, \mathrm{EC}=\mathrm{Ecuador}, \mathrm{MX}=$ Mexico, US = United States.

Table VIII-2. Parameters and goodness-of-fit for the PDF fitted (normal distribution and best-fitted distribution) for $D B H$ measurement by $D B H$ class in El Salto, Durango, Mexico.

Table VIII-3. Parameters and goodness-of-fit for the PDF fitted (normal distribution and best-fitted distribution) for $T H$ measurement by $T H$ class in El Salto, Durango, Mexico.

Table VIII-4. Parameters and goodness-of-fit for the PDF fitted (normal distribution and best-fitted distribution) for $D B H$ measurement made with Caliper by $D B H$ class in Göttingen, Germany.

Table VIII-5. Parameters and goodness-of-fit for the PDF fitted (normal distribution and best-fitted distribution) for $D B H$ measurement made with Tape by $D B H$ class in Göttingen, Germany.

Table VIII-6. Parameters and goodness-of-fit for the PDF fitted (normal distribution and best-fitted distribution) for $T H$ measurement made with clinometer by $T H$ class in Göttingen, Germany....

Table VIII-7. Parameters and goodness-of-fit for the PDF fitted (normal distribution and best-fitted distribution) for $T H$ measurement made with Vertex by $T H$ class in Göttingen, Germany.

Table VIII-8. AGB and uncertainty of the AGB calculation by plot using three scenarios for the measurement uncertainty estimation ( $N D n, N D n C, R M S D)$ in El Salto, Durango, Mexico.

Table VIII-9. Repeated-measures ANOVA, with correction for sphericity, applied to three scenarios (NDn,

$N D n C, R M S D$ ) of measurement uncertainty estimation in AGB calculation in El Salto, Durango, Mexico.. 143 Table VIII-10. Pairwise comparison using paired T- Test, with p-value Holm's correction, for three scenarios $(N D n, N D n C, R M S D)$ of AGB uncertainty estimation in El Salto, Durango, Mexico.

Table VIII-11. Uncertainty of the AGB calculation by plot using the estimation with all sources of measurement error $(N D n C)$ and by source $(N D n C a, N D n C b, N D n C a b, N D n C c)$ in El Salto, Durango, Mexico.

Table VIII-12. Repeated-measures ANOVA, with correction for sphericity, applied to five scenarios (NDnC, $\mathrm{NDnCa}, \mathrm{NDnCb}, \mathrm{NDnCab}, \mathrm{NDnCc}$ ) of measurement uncertainty estimation in AGB calculation in El Salto, Durango, Mexico.

Table VIII-13. Pairwise comparison using paired T-Test, with p-value Holm's correction, for five scenarios $(\mathrm{NDnC}, \mathrm{NDnCa}, \mathrm{NDnCb}, \mathrm{NDnCab}, \mathrm{NDnCc})$ of AGB uncertainty estimation in El Salto, Durango, Mexico.

Table VIII-14. Uncertainty of the AGB calculation by plot using seven scenarios for the measurement uncertainty estimation ( $M C N D n, M C N D n C, M C B D, M C B D C, N D n, N D n C, R M S D$ ) in El Salto, Durango, Mexico.

Table VIII-15. Repeated-measures ANOVA with correction for sphericity applied to seven scenarios ( $M C N D n, M C N D n C, M C B D, M C B D C, N D n, N D n C, R M S D)$ of measurement uncertainty estimation in AGB calculation in El Salto, Durango.

Table VIII-16. Pairwise comparison using paired T-Test, with p-value Holm's correction, for seven scenarios (MCNDn, $M C N D n C, M C B D, M C B D C, N D n, N D n C, R M S D)$ of AGB uncertainty estimation in El Salto, 
Table VIII-17. AGB uncertainty estimation by plot with Monte-Carlo simulation Method using non-sampling uncertainty estimation sources ( $M C C, M C C a, M C C b, M C C c)$ in El Salto, Durango, Mexico....................... 146 Table VIII-18. Repeated-measures ANOVA with correction for sphericity applied to four scenarios $(M C C$, $M C C a, M C C b, M C C c)$ of AGB uncertainty estimation in El Salto, Durango........................................ 146 Table VIII-19. Pairwise comparison using paired T-Test, with p-value Holm's correction, for four scenarios $(M C C, M C C a, M C C b, M C C c)$ of AGB uncertainty estimation in El Salto, Durango Mexico.....................146 Table VIII-20. AGB calculation by Plot using three scenarios for the measurement uncertainty estimation

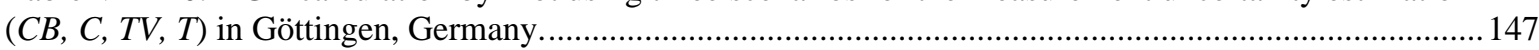
Table VIII-21. Repeated-measures ANOVA with correction for sphericity applied to four scenarios $(C B, C$, $T V, T)$ of AGB estimation in Göttingen, Germany.

Table VIII-22. Pairwise comparison using paired T-Test, with p-value Holm's correction, for three scenarios

$(C B, C, T V, T)$ of AGB estimation in Göttingen, Germany.....

Table VIII-23. Uncertainty of the AGB calculation by plot using four scenarios for the measurement

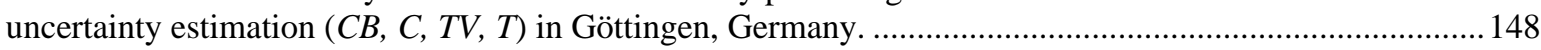
Table VIII-24. Repeated-measures ANOVA with correction for sphericity applied to four scenarios $(C B, C$, $T V, T)$ of AGB uncertainty estimation in Göttingen, Germany. 148 Table VIII-25. Pairwise comparison using paired T-Test, with p-value Holm's correction, for four scenarios $(C B, C, T V, T)$ AGB uncertainty estimation in Göttingen, Germany. 148 Table VIII-26. AGB and uncertainty of the AGB calculation by plot using five scenarios for measurement uncertainty estimation ( $C B, C B m e a n, C B N D n, C B N D n C, C B m o d)$ in Göttingen, Germany. 149 Table VIII-27. Repeated-measures ANOVA with correction for sphericity applied to five scenarios $(C B$, CBmean, $C B N D n, C B N D n C, C B m o d)$ of AGB uncertainty estimation in Göttingen, Germany. 149 Table VIII-28. Pairwise comparison using paired T-Test, with p-value Holm's correction, for five scenarios $(C B, C B m e a n, C B N D n, C B N D n C, C B m o d)$ of AGB uncertainty estimation in El Salto, Durango Mexico... 149 Table VIII-29. Uncertainty of the AGB calculation by plot using six scenarios for the measurement uncertainty estimation (TV, TVmean, TVNDn, TVNDnC, TVmod) in Göttingen, Germany. 150 Table VIII-30. Repeated-measures ANOVA with correction for sphericity applied to five scenarios (TV, TVmean, TVNDn, TVNDnC, TVmod) of AGB uncertainty estimation in Göttingen, Germany. 150 Table VIII-31. Pairwise comparison using paired T-Test, with p-value Holm's correction, for seven scenarios (TV, TVmean, TVNDn, TVNDnC, TVmod) of AGB uncertainty estimation in Göttingen, Germany............. 150 Table VIII-32. Uncertainty of the AGB calculation by plot using three scenarios for the measurement uncertainty estimation (TV, TVExp, TVNexp) in Göttingen, Germany

Table VIII-33. Repeated-measures ANOVA with correction for sphericity applied to three scenarios $(T V$, TVExp, TVNexp) of AGB uncertainty estimation in Göttingen, Germany.

Table VIII-34. Pairwise comparison using paired T-Test, with p-value Holm's correction, for three scenarios (TV, TVExp, TVNexp) of AGB uncertainty estimation in Göttingen, Germany.................................... 151 Table VIII-35. Repeated-measures ANOVA with correction for sphericity applied to three scenarios $(C B B D$, $C B B D C, C B m o d, C B N D n)$ of AGB uncertainty estimation in Göttingen, Germany.

Table VIII-36. Pairwise comparison using paired T-Test, with p-value Holm's correction, for four scenarios ( $C B B D, C B B D C, C B m o d, C B N D n)$ of AGB uncertainty estimation in Göttingen, Germany.

Table VIII-37. Repeated-measures ANOVA with correction for sphericity applied to four scenarios (TVBPDF, TVBDC, TVmod, TVNDn) of AGB uncertainty estimation in Göttingen, Germany.................152 Table VIII-38. Pairwise comparison using paired T-Test, with p-value Holm's correction, for four scenarios (TVBPDF, TVBDC, TVmod, TVNDn) of AGB uncertainty estimation in Göttingen, Germany ...................152 Table VIII-39. Repeated-measures ANOVA with correction for sphericity applied to six scenarios (Exp, Nexp, CBmod, CBNDn, TVmod, TVNDn) of AGB uncertainty estimation in Göttingen, Germany. .......................153 Table VIII-40. Pairwise comparison using paired T-Test, with p-value Holm's correction, for six scenarios (Exp, Nexp, CBmod, CBNDn, TVmod, TVNDn) of AGB uncertainty estimation in Göttingen, Germany...... 153 Table VIII-41. Result of Monte-Carlo simulation Method on the contribution by uncertainty source to the total uncertainty in AGB estimation in Göttingen, Germany. 154 Table VIII-42. Basal area $(G)$ and AGB $(W)$ estimation made by classes of $D B H$ and $T H$, in four datasets of Durango temperate forest. Estimation made with data of two periods of MNFI 2004-2009 and 2009-2014. 156 Table VIII-43. Temperate forest surface by basal area $(G)$ class in Durango, Mexico. . 162 Table VIII-44. Temperate forest surface by AGB $(W)$ class and amount of $W$ stored by class in Durango, Mexico. 



\section{List of abbreviations}

\begin{tabular}{|c|c|}
\hline AGB & Aboveground biomass \\
\hline $\mathrm{AM}$ & Allometric model to predict AGB \\
\hline $\mathrm{BD}$ & Best distribution fitted to measurement errors \\
\hline $\mathrm{BDC}$ & Best distribution fitted to measurement errors by classes of $\mathrm{DBH}$ and $\mathrm{TH}$ \\
\hline $\mathrm{CB}$ & Tree measurements made with Caliper $(\mathrm{DBH})$ and Blume-Leiss $(\mathrm{TH})$ \\
\hline $\mathrm{CO}_{2}$ & Carbon Dioxide \\
\hline CONAFOR & Comisión Nacional Forestal=National Forest Commission in Mexico \\
\hline $\mathrm{CV}$ & Coefficient of variation \\
\hline $\mathrm{DBH}$ & Diameter at breast height $(130 \mathrm{~cm})$ \\
\hline FAO & Food and Agriculture Organization of the United Nations \\
\hline FRA & Global Forest Resources Assessment \\
\hline GHG & Greenhouse gases \\
\hline GOF & Goodness-of-fit \\
\hline GUM & Guide to the expression of uncertainty in measurement \\
\hline GUM Method & Error propagation method using the GUM \\
\hline INEGI & $\begin{array}{l}\text { Instituto Nacional de Estadistica y Geografía = National Institute of Statistics } \\
\text { and Geography in Mexico }\end{array}$ \\
\hline IPCC & Intergovernmental Panel on Climate Change \\
\hline LEP & Law of Error Propagation \\
\hline MCM & Monte-Carlo simulation Method \\
\hline MNFI & Mexican National Forest Inventory \\
\hline mod & Linear regression model fitted to measurement errors \\
\hline MSE & Mean square error \\
\hline MRV & Measurement, Reporting, Verification \\
\hline NDn & Normal distribution fitted to measurement errors \\
\hline $\mathrm{NDnC}$ & Normal distribution fitted to measurement errors by classes of $\mathrm{DBH}$ and $\mathrm{TH}$ \\
\hline NFI & National Forest Inventory \\
\hline PDF & Probability Density Function \\
\hline PV & Predictor variables \\
\hline RMSD & Root mean square deviation \\
\hline RSS & Residual sum of squares \\
\hline SCF & Stratum of conifer forest into the temperate forest \\
\hline SE & Sampling error \\
\hline SMF & Stratum of mixed forest (conifer and oak) into the temperate forest \\
\hline $\mathrm{SMO}$ & Sierra Madre Occidental $=$ Western Mother Mountains \\
\hline SOF & Stratum of oak forest into the temperate forest \\
\hline SRS & Simple random sampling \\
\hline $\mathrm{TF}$ & Temperate forest \\
\hline $\mathrm{TH}$ & Total height \\
\hline TV & Tree measurements made with Tape (DBH) and Vertex IV (TH) \\
\hline UNFCCC & United Nations Framework Convention on Climate Change \\
\hline uAM & Uncertainty of AGB prediction with allometric model \\
\hline $\mathrm{uDBH}$ & Uncertainty of DBH measurement \\
\hline uMes & Uncertainty of measurements \\
\hline uNSplot & Uncertainty of non-sampling at plot-level \\
\hline uStand & Uncertainty at stand-level \\
\hline
\end{tabular}


uTH

uTree

VI
Uncertainty of TH measurement

Uncertainty of non-sampling at tree-level

Vegetation Index 


\section{Chapter I: Introduction}

\section{I.1 Background}

\section{I.1.1 Origins and present of uncertainty}

The term uncertainty is better known in the social sciences (Wakeham, 2015), especially when it relates to making a decision (Marchau et al., 2019), when referring to the knowledge or lack of knowledge (Pérez-Hernández, 2012) or incomplete information (Ascough et al., 2008).

Over the course of history, analogies of uncertainty have been developed, and uncertainty has even been considered as "what can not be explained". Frank Knight in 1921, defined risk as part of a process "calculable", while uncertainty he defined as "the unknown" (Marchau et al., 2019).

Between the 1970s and early 1990s uncertainty was synonymous with ambiguity, while in the late 1990s and until 2011 it was related to "ignorance or lack of knowledge"(Wakeham, 2015).

Recently, Marchau et al. (2019), arguing that “...uncertainty is a broader concept than risk”, defined five levels of uncertainty between total precision (determinism), and total ignorance (Figure I-1). Marchau et al. (2019) defined these levels based on the four aspects proposed by Walker et al. (2003): i) context or definition of the system, ii) the variables and relationships involved in the system, iii) the outcomes from the system, iv) the weights of the outcomes set by stakeholders.

\begin{tabular}{|c|c|c|c|c|c|}
\hline & Level 1 & Level 2 & Level 3 & Level 4 & Level 5 \\
\hline \multirow[t]{2}{*}{ Context } & & & & & \\
\hline & $\begin{array}{l}\text { A clear enough } \\
\text { future }\end{array}$ & Alternative futures & $\begin{array}{l}\text { A few plausive } \\
\text { futures }\end{array}$ & $\begin{array}{l}\text { Many plausive } \\
\text { features }\end{array}$ & $\begin{array}{l}\text { Unknow } \\
\text { future }\end{array}$ \\
\hline System model & $\begin{array}{l}\text { A single } \\
\text { (deterministic) } \\
\text { system model }\end{array}$ & $\begin{array}{l}\text { A single } \\
\text { (stochastic) system } \\
\text { model }\end{array}$ & $\begin{array}{l}\text { A few alternative } \\
\text { system models }\end{array}$ & $\begin{array}{l}\text { Many alternative } \\
\text { system model }\end{array}$ & Unknown \\
\hline System outcomes & $\begin{array}{l}\text { A point estimate } \\
\text { for each outcome }\end{array}$ & $\begin{array}{l}\text { A confidence } \\
\text { interval for each } \\
\text { outcome }\end{array}$ & $\begin{array}{l}\text { A limited range } \\
\text { of outcomes }\end{array}$ & $\begin{array}{l}\text { A wide range of } \\
\text { outcomes }\end{array}$ & Unknown \\
\hline Weights & $\begin{array}{l}\text { A single set of } \\
\text { weights }\end{array}$ & $\begin{array}{l}\text { Several sets of } \\
\text { weights, with a } \\
\text { probability attached } \\
\text { to ecah set }\end{array}$ & $\begin{array}{l}\text { A limited range } \\
\text { weights }\end{array}$ & $\begin{array}{l}\text { A wide range of } \\
\text { weights }\end{array}$ & Unknown \\
\hline
\end{tabular}

Figure I-1. Levels of uncertainty (source: Marchau et al., 2019)

The uncertainty in aboveground biomass (AGB) estimation is set at Levels 1 and 2 of Figure I-1. According to Marchau et al. (2019), these two levels encompass scientific work in the natural sciences where an emphasis on reducing uncertainty in the result is placed. This can be achieved through acquiring more information about the process or through stochastic variation by statistical analysis. 


\section{I.2 Aboveground biomass}

\section{I.2.1 Aboveground biomass (AGB) definition}

Biomass is the matter of living or dead organisms in a specific place expressed in weight by unit area or by unit volume (GTOS \& FAO, 2009). Plant biomass relates to the individuals or parts of individuals found on the soil, such as trees, crops, shrubs, herbs; and parts found in the soil or below the surface, i.e., roots (FAO, 2006). AGB in trees, is all living matter over the soil comprising trunk, stump, branches, bark, seed, and leaves (FAO, 2006; IPCC, 2006).

Biomass in plants is produced through photosynthesis, where plants generate organic substances from the absorption of atmospheric carbon dioxide $\left(\mathrm{CO}_{2}\right)$ and water in the presence of light, storing it as dry matter (BUN-CA, 2002). When plant biomass is burned, $\mathrm{CO}_{2}$ (BUN-CA, 2002; Gibbs et al., 2007) and other trace gases and aerosols are emitted into the atmosphere (GTOS \& FAO, 2009). $\mathrm{CO}_{2}$ is one of the main greenhouse gases (GHG) and has increased by more than $30 \%$ in concentration in the atmosphere over the last century (CONAFOR, 2012a; IPCC, 2013). Reports from international panel experts conclude that there is a probability close to certainty that humans influence climate change through energy, industry, forestry, transport and agriculture sectors (IPCC, 2013; PASCC, 2010).

Forest ecosystems have the capacity to absorb $\mathrm{CO}_{2}$ and store it as biomass for long periods of time depending on the lifetime of the corresponding organisms. Forests are therefore referred to as carbon sinks or reserves (IPCC, 2003), and cover about 30.6\% of the global land area (FAO, 2015). Since forests on their own cannot increase $\mathrm{CO}_{2}$ capture (Ma et al., 2019), forest plantations (Brancalion et al., 2019), forest management for wood products extraction (Profloresta, 2008; SEMARNAT, 2014), promoting the use of wood in construction (FAO, 2015), among other activities, increase the natural capacity of the forest to capture $\mathrm{CO}_{2}$. Also, future land-use change (LUC) scenarios combined with disturbance (wildfire, weather, insects, disease) have been evaluated, finding more AGB storage in the scenarios than in the undisturbed forest (Ma et al., 2019). With these dynamics in the forest, it is important to evaluate their AGB stocks and the changes associated with these AGB stocks: deforestation, reforestation, increase in agricultural areas, increase in urban areas, among others (IPCC, 2003, 2015).

\section{I.2.2 Aboveground biomass estimation}

\section{I.2.2.1 Direct method of measurement or tree-level observations}

The direct method (Figure I-2) involves measuring a standing tree and after felling, drying and weighing to get the dry matter or AGB (Picard et al., 2012; Shi \& Liu, 2017). If the AGB of more than one tree is measured with this method, a prior analysis is performed to stratify the area of interest and calculate the sample size of trees that will be subject to the process (Picard et al., 2012).

This method is a costly and elaborated process that requires a protocol (Magnussen \& Reed, 2015) that clearly sets out a sequence of actions to follow in field data collection and chain of custody to avoid systematic errors. The "Manual for building tree volume and biomass allometric equations" represents an effort by CIRAD and FAO to set best practices for direct method estimations (Picard et al., 2012). 


\section{I.2.2.2 Indirect methods of estimation}

\section{I.2.2.2.1 Observations at plot-level}

The ground based measurement of biomass via the direct method can not be achieved over large areas, due to cost and because it is impractical since the purpose is to know the amount of AGB stored (Magnussen \& Reed, 2015). In this case, an indirect estimation method is applied (Figure I2 ), which uses data from standing trees as predictive variables to calculate regression equations based on allometric relationships (Magnussen \& Reed, 2015; Picard et al., 2012; Shi \& Liu, 2017). These equations are allometric models and describe the relationship of AGB with standing tree variables (GTOS \& FAO, 2009; Picard et al., 2012). If AGB allometric models were not available, then conversion factors can be used to transform the tree volume into AGB (GTOS \& FAO, 2009). Once calculating the AGB by tree, this is aggregated or grouped by characteristics of interest such as diameter class, height class, species, genus, among others (Magnussen \& Reed, 2015). Once the AGB has been added at the plot-level, the estimators for the study area are calculated, considering the sampling design applied to the forest inventory (McRoberts et al., 2015).

Direct measurement

\section{AGB tree-level (Measuring,} felling, drying, weighing)

Fitting allometric models $A G B=f(D B H, T H$, crown diameter, wood density)

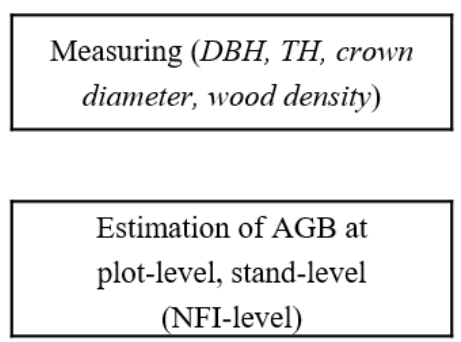

(NFI-level)
Indirect estimation

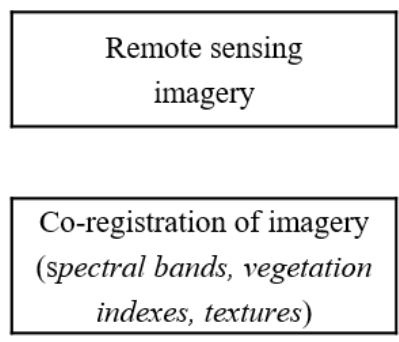

AGB maps

Figure I-2. Direct and indirect methods of AGB estimation.

\section{I.2.2.2.2 Extrapolation of the AGB estimation}

Under plot-level AGB estimation, there are "gaps" (the area not selected in the sampling) and remote areas without field data, where remote sensing techniques can be employed to estimate the AGB (Hyde et al., 2006; Sarker \& Nichol, 2011). As the advantages of AGB estimation with remote sensing, are included the strong correlation between spectral data and vegetation parameters, the repeatability of data collection and the availability of global image coverage (Dengsheng Lu, 2006).

AGB indirect estimation with National forest inventory (NFI) data is reported every 5 to 10 years, corresponding to the time used for field data collection and data analysis (Sousa et al., 2017). The monitoring of AGB (Figure I-2), from satellite imagery, is an indirect method that can increase the estimation frequency of AGB and can be complementary to NFI data (GTOS \& FAO, 2009).

There are studies which use estimates of the AGB obtained with field-measured dasometric information (as a response variable) to calibrate images from sensors such as Landsat (Gizachew et al., 2016; Vargas-Larreta et al., 2017), Ikonos 2 (Phua et al., 2012), Radar (Sinha et al., 2015), ALS 
(Peuhkurinen et al., 2008), Sentinel (Alboabidallah et al., 2017) or a combination of sensors for estimating AGB in larger areas and in shorter periods of time than the NFI (GFOI, 2016).

Since optical sensors have a saturation effect on AGB estimation (GTOS \& FAO, 2009), the texture has been a property of the images that has been included to increase the range of AGB (Fuchs et al., 2009; Lu \& Batistella, 2005; Sarker \& Nichol, 2011) estimation from the discrimination of the levels of tones related to regions or objects (Haralick et al., 1973).

\section{I.3 Errors and uncertainties}

\section{I.3.1 Definition of uncertainty}

In the scientific domain, according to the Joint Committee for Guides in Metrology (JCGM, 2010), the uncertainty is a parameter related to the result of a measurement that describes the spread of values that can be reasonably assigned to the measurement. Other authors complement this definition as the range in which results are expected, including the probability with which this range was obtained (Kallner, 2001; Taylor, 1997).

The method to analyze the uncertainties associated with estimation is the propagation of uncertainties (JCGM, 2010; Taylor, 1997). This method divides the problem into stages, quantifies the uncertainties separately, and then combines them to get the total uncertainty (Kallner, 2001). The division into stages is due to the identification of the sources of uncertainty that, presumably or with information from previous studies, contribute most to the total uncertainty (Schmid \& Lazos Martínez, 2000). The quantification of uncertainty usually embraces the assignment of value plus its distribution (Schmid \& Lazos Martínez, 2000).

\section{I.3.2 Reporting uncertainty}

Ascough et al. (2008) point out the importance of reporting uncertainty in all types of empirical studies in the environmental and ecological context, emphasizing the relationship between understanding the uncertainty with the quality of decision-making. In the forestry context, Kauffman et al. (2013) mentioned that by including uncertainty analysis in aboveground biomass (AGB) estimation, reference was made to the precision of the reported information. According to the Global Terrestrial Observing System and Food and Agriculture Organization (GTOS \& FAO, 2009), this refers then also to the reliability of the information.

Research on forest biomass, including uncertainty analysis has increased, given the development of government policies and international negotiations about forest response and climate change (Shi \& Liu, 2017). As a national strategy, the Mexican government has promoted the development of public policy instruments that consider strategies in the economic/climate sectors (SEMARNAT \& SHCP, 2009), and their relationship with the technical parameters in forest emissions (CONAFOR, 2017b). These policy instruments contain clear methodologies of uncertainty analysis and include uncertainty estimates in the results to be obtained (CONAFOR, 2014b).

\section{I.3.3 Errors in National forest inventories}

Different measurement methods are used in NFIs to get observations to record the current state of the forest (CONAFOR, 2017a; Kleinn et al., 2015). The best methods improve the acquisition 
accuracy of the measurements while reducing the acquisition time (Diéguez Aranda et al., 2005; Kershaw Jr., Ducey, Beers, \& Husch, 2017). Therefore, data collected from NFIs is objectively prone to error. Across this thesis work, the word "error" will not be used as a synonym for "mistake" or "carelessness" (Gil \& Rodríguez, 2001) rather as the uncertainty of measurement/estimation (Taylor, 1997). As before defined Section I.3.1, an error will be considered as the residual variability associated with the measurement or estimation, thus describing the dispersion values logically attributed to AGB measurement (JCGM, 2010).

The total error of estimation in NFIs involves different components, including sampling and nonsampling error (FAO, 1981; Kleinn et al., 2015; United Nations, 2008). The final report of the NFI in Mexico, for example, included sampling error as the source of all observed variations in the variables considered (number of trees, basal area, volume, biomass, etc.) without reporting nonsampling errors (CONAFOR, 2012b, 2017a).

\section{I.3.4 Sampling error}

NFI plots sample the landscape to measure variables of interest, from which the parameters of the target population are estimated (Köhl et al., 2006). However, the estimators are subject to error due to the sampling design applied (Kershaw Jr. et al., 2017), meaning that the error would not be present if the entire population was included in the observations (Gormanson et al., 2017; McRoberts et al., 2015). This error is referred to as sampling error. The sampling error of probability samples is reported as the standard error of the mean $(S E)$, coefficient of variation $(C V)$ or the confidence interval (Köhl et al., 2006; United Nations, 2008), of a given variable e.g. volume, AGB, etc. (FAO, 1981; Köhl et al., 2006; McRoberts, Næsset, et al., 2015). Considering that SE measures the precision of the estimate, sampling error is related to the sample size and is therefore intrinsically associated with the time spent doing fieldwork and budgets allocated to the inventory (Kershaw Jr. et al., 2017; United Nations, 2008).

McRoberts et al. (2015) show the use of the $C V$ to be effective when comparing across sampling designs; whereby the differences between sampling designs were given by sample size and interplot distances. Similarly, an optimal sampling error is defined by the smallest $S E$ per sampling design given the costs assigned to the inventory (United Nations, 2008). Tomppo et al. (2010) compared 31 European countries, 3 Asian (China, Japan, and Republic of Korea), 3 on the American continent (Brazil, Canada, and USA) and New Zealand from 1992 to 2009 and reported NFI sampling errors for wood volume by $S E$ ranging from $0.46 \%$ (USA) to $7.14 \%$ (Ireland). However, in this comparison, there were inconsistencies in the definition of wood volume as the height of $D B H(1.3-1.5 \mathrm{~m})$, minimum $D B H(0-12.5 \mathrm{~cm})$, elements sampled other than standing trees (stumps, branches and/or dead wood), among others. More recent results to those reported by Tomppo et al. (2010) can be accessed online in most countries, and show an improvement in the estimation precision of wood volume with $S E$ of $0.31 \%$ in the USA and $2.17 \%$ Ireland (https://www.fia.fs.fed.us/; https://www.agriculture.gov.ie/nfi/). In Mexico, the first repeat survey of the NFI (2009-2014), reported for volume a SE of 3.2 - $4 \%$ (CONAFOR, 2017a). These results are consistent with those obtained in the first NFI (2004-2009) where the volume was estimated with 3.2 - $4 \%$ of $S E$ (CONAFOR, 2012c). The AGB was reported only for the temperate forest in NFI (2009-2014) with $2.6-3.3 \%$ of $S E$. 


\section{I.3.5 Non-sampling errors: measurement errors}

The goals established in an NFI, such as timber supply, biodiversity, REDD+, etc., determine the variables measured during fieldwork (Kleinn, 2017; Kleinn et al., 2015). Trees are the object, where the measurements are made and the values of the variables of interest registered. Tree measurements are made assuming geometric forms like the tree cross-section (circle, oval), tree form (cone, frustum cone), or tree crown (circle, oval) (Kershaw Jr. et al., 2017; Matérn, 1956) and thus inherently carry an error in their magnitudes. Thus, it is important to estimate measurement uncertainty, so as to determine the quality of the measurement. Such a result can be the source of information for another project or for a decision-making process (Pérez-Hernández, 2012).

There are two important components of measurement error, systematic and random errors (Taylor, 1997; United Nations, 2008). Both systematic and random measurement errors are independent of each other and hence should be quantified independently. Figure I-3 shows that the total error can be quantified as the hypotenuse, of the Pythagoras' theorem, joining both error axes. Considering that it is not possible to avoid random error in any measurement (Taylor, 1997), one can posit that smaller total error can be achieved, when systematic error reduces and as systematic error tends to zero, the total error equals to the random error.

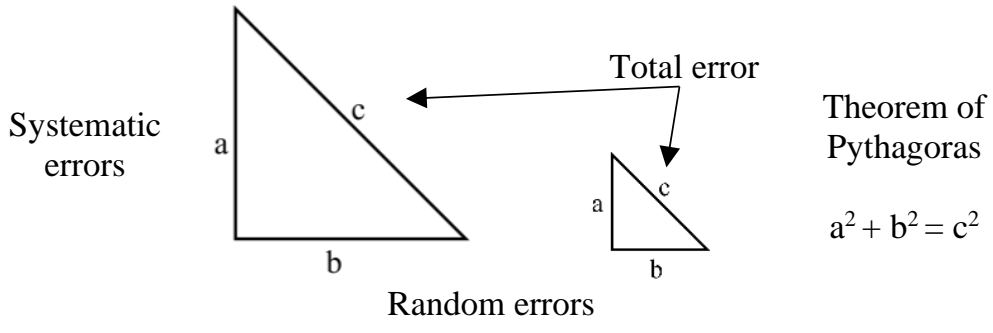

Figure I-3. Total error in the measurement as a product of systematic errors and random errors. Modified from source (United Nations, 2008).

Practically, measurement errors arise from the faulty or incorrect use of measurement devices e.g. from device calibration (Diéguez Aranda et al., 2005) or dependent on the accuracy of the measurement devices (Gil \& Rodríguez, 2001). Incorrect use of measurement devices by staff is often related to either measurement criteria or staff capacity use the availed forest inventory devices (Canavan \& Hann, 2014; Diéguez Aranda et al., 2005).

\section{I.3.6 Non-sampling errors: prediction errors due to allometric models}

The allometric model to estimate the AGB is obtained from regression analysis (Picard et al., 2012). The AGB is the result of statistical relationship with tree variables such as $D B H$ (Avendaño Hernandez et al., 2009; Návar, 2009), TH (Foroughbakhch et al., 2006; Vargas-Larreta et al., 2017), crown diameter (Návar et al., 2004), wood density (Martinez-Yrizar et al., 1992; Wiemann \& Williamson, 2013). This model can be applied to other standing trees located in the same site, where the model was obtained, or in areas with similar site-characteristics (GTOS \& FAO, 2009); or applying a scientific approach as a suitability check of the model validating the prediction uncertainty precision to select a AGB model (Pérez-Cruzado et al., 2015). Then, site-characteristics is a potential source of bias if not considered in the selection of model (Pérez-Cruzado et al., 2015), 
because it is related to climatic conditions or soil types and these are associated with tree growth and with accumulation of AGB (GTOS \& FAO, 2009; Picard et al., 2012; Shi \& Liu, 2017).

As a result of the regression model, statistics that represent the adjustment of the equation for the AGB and the uncertainty of the model, based on the tree measurement variable(s) are obtained (GTOS \& FAO, 2009). The most commonly used indicator is the coefficient of determination $\left(R^{2}\right)$, representing the quality of the model to be replicated and the proportion of variation of the results that can be explained by the model (Ayala Gallego, 2015; Mehtätalo, 2013). Mean square error $(M S E)$ is a measure of the difference between the estimator and what is estimated. The square root of the MSE (RMSE), is the parameter of precision associated with the model assuming a constant variance of the error (Cochran, 1977). Two more indicators are obtained from the regression analysis, the error in prediction of the mean used to estimate the confidence interval of the regression, and the error in prediction of an individual used to estimate the prediction interval of the regression (Draper \& Smith, 1998). Only 40 of 478 studies in AGB allometric equations for Mexican trees forest, reported the parameters related to the uncertainty: RMSE or SE (Rojas-García et al., 2015a).

The allometric models of AGB are generated under different criteria related to the stand characteristics, geographic area and the delimitation of classes according to the size range of the trees to be characterized. Based on stand characteristics, the allometric model could be made for species specific (Vargas-Larreta et al., 2017), genus (Méndez González et al., 2012) or group of species (Búrquez et al., 2010). The geographic area criterion is related to allometric models generated with information of one stand (Shi \& Liu, 2017), a group of stands (Méndez González et al., 2012), a location (Návar et al., 2004) or a region (Shi \& Liu, 2017; Vargas-Larreta et al., 2017); considering those areas are referred to the physiographic conditions of the area (Shi \& Liu, 2017). The models have a range of validity whose extreme values are the minimum and maximum values of the variables used when calculating the model; if we use the model to predict AGB outside this range of values, estimation biases may occur (Picard et al., 2012).

\section{I.3.7 Quality control of data}

The quality control of data is due to the handling of data records in the field (United Nations, 2008), transfer of field forms observations to electronic media (Kershaw Jr. et al., 2017), and the statistical process of data in computer programs (Canavan \& Hann, 2014). Therefore, it is important to implement verification mechanisms at each step involving data transfer, to have a reliable database for information analysis (United Nations, 2008). With an emphasis on the proper training of the work teams in the process of collection and storage of field information (FAO, 1981; United Nations, 2008).

To ensure data quality, NFI's have been implementing protocols to assess the quality of information recorded (Tomppo et al., 2010). The protocols include data electronic storage, double review in fieldwork, plausibility checking (included in the storage equipment), automatic verification on the central server (logical check) and verification of 5-10\% of the sampled plots. 


\section{I.4 Error propagation}

\section{I.4.1 General observations of error propagation}

When a predictor variable (or variables) is used to estimate AGB with a model, and the measurement error was calculated for the predictor variable, the AGB error per tree will be estimated through error propagation or uncertainty propagation based on the contribution of the predictor variable into the model (Hughes \& Hase, 2010). Uncertainty propagation is also used in the aggregation process of AGB from tree-level to plot-level and continuing until stand-level, for the variable underestimation (Chave et al., 2004).

As before indicated, for the estimation of AGB with NFI information in a ground-based approach (Figure I-4), the first source of error is the measurement $s_{m}$. The second source of error incorporates the uncertainty with the error of the allometric model $s_{A M}$. The third source of uncertainty is due to the design of sampling through sampling error $s_{S E}$. At the end of this process is estimated the total uncertainty $s_{A G B}$ as a result of the error propagation (Shi \& Liu, 2017).

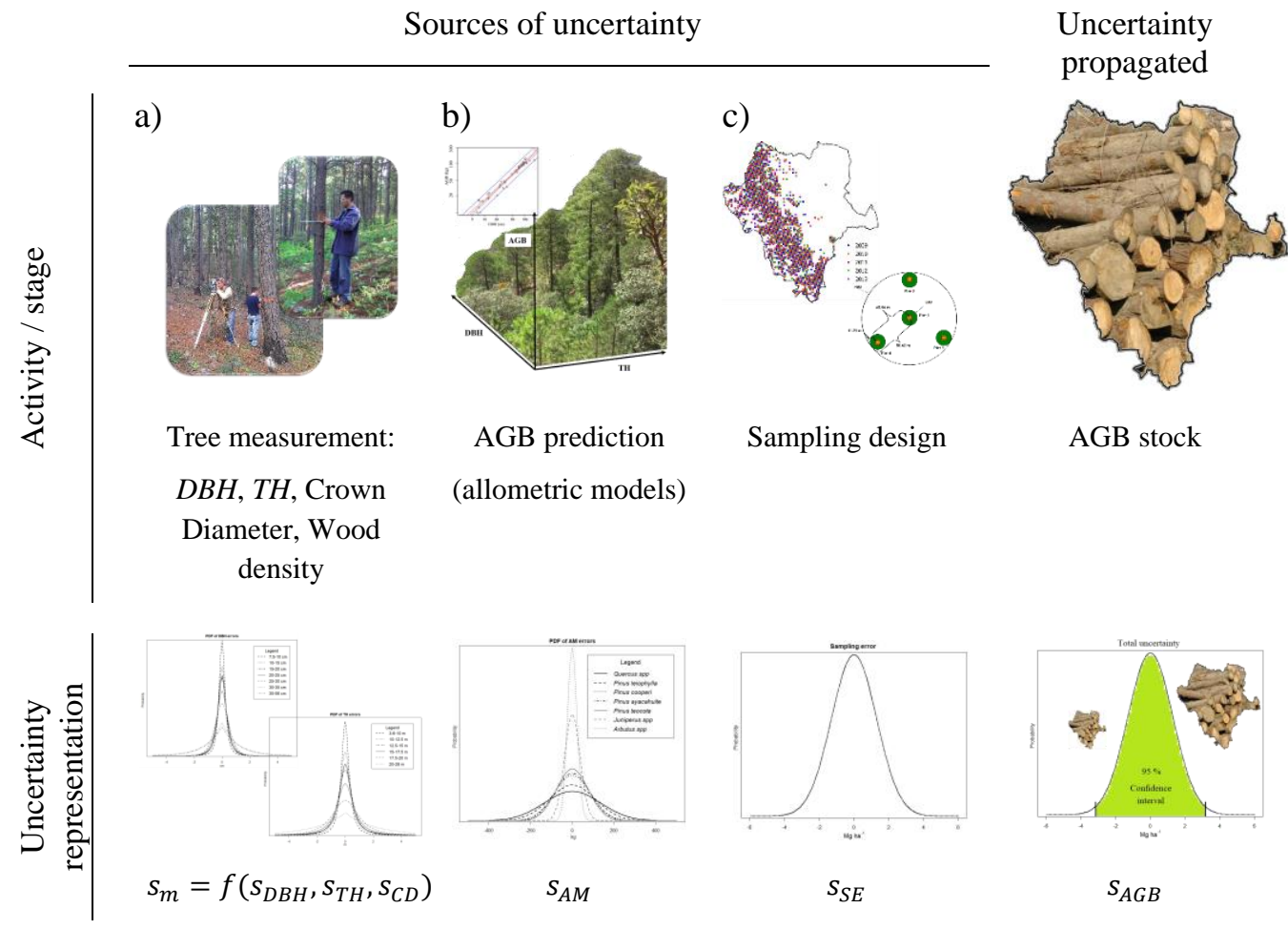

Figure I-4. Sources of uncertainty evaluated in the error propagation of AGB using NFI fieldwork data.

\section{I.4.2 GUM Method of error propagation}

Error propagation calculated by the application of the Law of Error Propagation (IPCC, 2003) is described in the Guide to the expression of Uncertainty in Measurement (GUM), first published in 1993 by the Joint Committee for Guides in Metrology (JCGM, 2010; Pérez-Hernández, 2012) and is reported as a GUM Method. To apply the GUM Method in this thesis, the following conditions must be present: the process is applied to a single response variable of the mathematical allometric model; the allometric model must be explicit; mathematical expectations and uncertainties can be 
calculated from the explanatory variables. To propagate uncertainties, the GUM Method establishes the procedure summarized in the following steps and applied to the variable AGB (Farrance \& Frenkel, 2014; JCGM, 2010; Schmid et al., 2000):

i. Specify the measurand to calculate AGB. The physical model or set of assumptions about the measurement is defined, allometric models approximate AGB in the forest and through this calculation, AGB is associated with predictor variable(s).

ii. Identify the sources of uncertainty. Measurement error, model error to estimate AGB and sampling error.

iii. Quantify uncertainty components or standard uncertainties. There are two types of evaluations. In type A evaluation, uncertainty is described by analyzing the probability distribution function of measurement error, and therefore, the distribution parameters associated with each error source. Type B evaluation, error behavior is obtained through calibration certificates, literature, regulations or previous studies; the allometric models to estimate AGB in NFI.

iv. Calculate the value of the AGB.

v. Calculate the combined uncertainty. The Law of Error Propagation is applied to combine standard uncertainty from each error source.

vi. $\quad$ Calculate the expanded uncertainty. This is the confidence interval for the AGB estimate and is calculated using the combined uncertainties multiplied by a coverage factor $(\mathrm{k})$.

The explanation of the steps for error propagation by the GUM Method will be discussed in more detail in Chapter III of this thesis.

\section{I.4.3 Monte-Carlo simulation Method for error propagation}

Monte-Carlo simulation Methods ( $M C M$ ) are defined as numerical (Hughes \& Hase, 2010), computer-based (Castro Quilantán et al., 2010) or experimental probabilistic technics (Basil et al., 2001), used to estimate probabilities, mean values, confidence intervals, using a sequence of random or pseudorandom numbers (Hughes \& Hase, 2010). Due to its relation to randomness, the method has taken its name from the principality of Monte-Carlo is known for casinos where the basic principle of its games is to get the result with low probability commonly stated as "by chance" (Martínez, 2003).

$M C M$ has been applied to different fields of science to solve many of the problems associated. Error limits in accidentology (Martínez, 2003), risk estimation (Azofeifa, 2005), uncertainty in flow measurement (Basil et al., 2001), evaluation of measurement uncertainty of pharmaceutical certified reference material (Rocha \& Nogueira, 2012), estimate the uncertainty of airflow measurement (Sediva et al., 2015). In natural resources, the uncertainty assessment has been applied in ecosystem budget calculations (Yanai et al., 2010), individual tree volume estimation (McRoberts, Tomppo, et al., 2015; McRoberts \& Westfall, 2014, 2016), plot-based estimates of carbon stock (Holdaway et al., 2014), among others.

The $M C M$ for error propagation is conceptually simple. The AGB estimate is evaluated repeatedly by including the uncertainty of the explanatory variables $(E V)$ in the estimate. This uncertainty is randomly selected from the $E V$ probability distribution error, calculating values higher or lower than a mean value of the $E V$ (Ogilvie, 1984; Rees, 1984; Yanai et al., 2010). If the AGB estimate has more than two $E V s$, the variance-covariance matrix structure in the joint probability distributions should be considered, which measures the joint variability of EVs (Ogilvie, 1984; Press 
et al., 2007). After repeating the process for numerous times, the result defines the probability distribution of the propagated error (Yanai et al., 2010). The $M C M$ procedure provides simulationbased approximations to the propagated uncertainty without the need of differential equations associated with the GUM Method (Farrance \& Frenkel, 2014).

\section{I.5 Justification of this study}

\section{I.5.1 Global level}

\section{I.5.1.1 Uncertainty in the estimation of $A G B$}

In 1988, IPCC activities began with the aim of carrying out comprehensive assessments associated with climate change (IPCC, 2003). To contribute to its main commitment in 1998 United Nations Framework Convention on Climate Change (UNFCCC) requested the IPCC to standardize the evaluation of GHG inventories, developing the Guidelines for national GHG inventories, which has been reinforced by the contributions of the National Greenhouse Gas Inventories Program (IPCC, 2006). This guide was developed for the Kyoto Protocol compliance (IPCC, 2003, 2006). The guide includes the standardized method to generate detailed information for each sector that contributes to climate change and the assessment of the uncertainty associated with each sector (IPCC, 2003). Houghton (2005) contributed to evaluating the uncertainty of forest biomass under different landuse change carbon flux scenarios (Figure I-5); three of the scenarios used for forest biomass estimation from the FAO Forest Resources Assessment reports (1980, 1990 and 2000). The difference between the evaluated scenarios of $0.95 \mathrm{PgC} \mathrm{yr}^{-1}$ was attributed to the uncertainty of the forest biomass estimate. Moreover, the result presented in the 2014 IPCC report indicated that the main cause of climate change was the emission of carbon dioxide $\left(\mathrm{CO}_{2}\right) \cdot \mathrm{CO}_{2}$ contributions associated with agriculture, forestry and other land-use (AFOLU) are the second in importance with a $24 \%$ contribution to the emission of GHG, only behind the energy sector with $34 \%$ (IPCC, 2014).

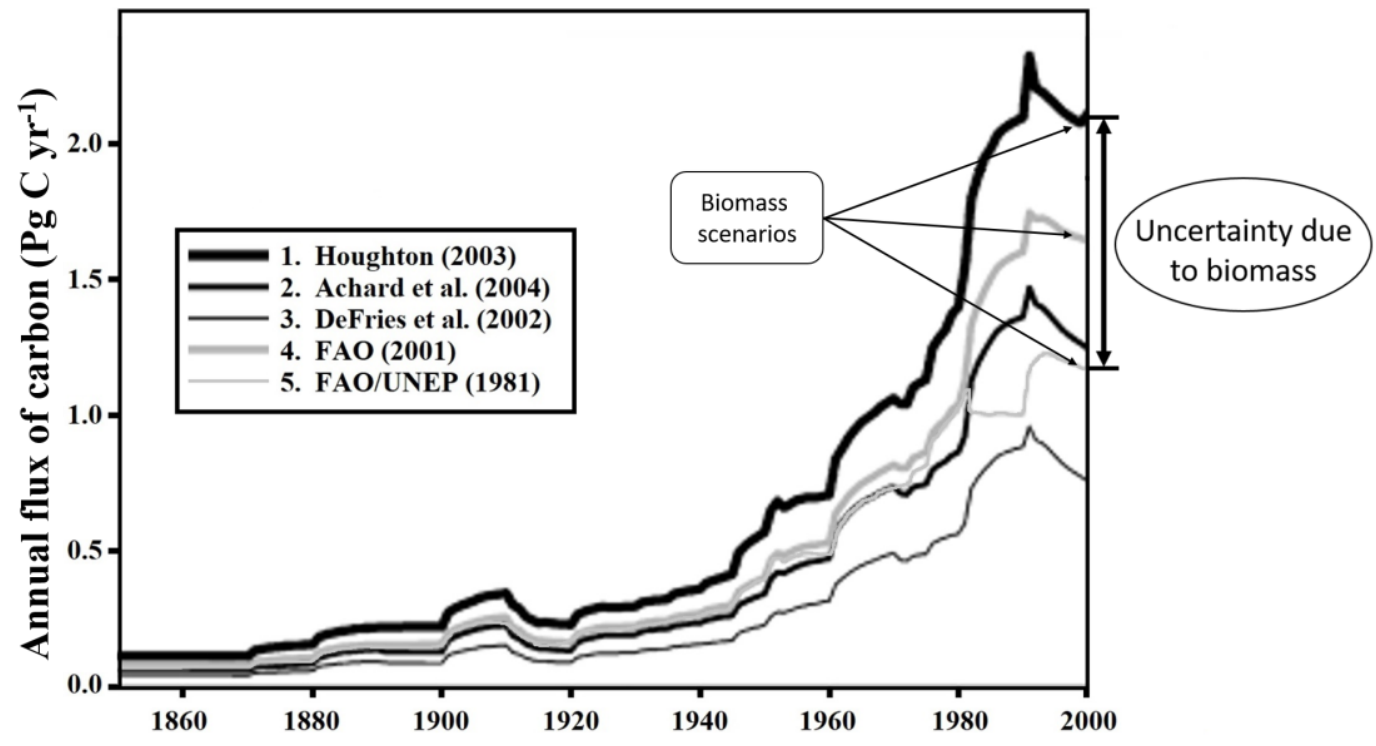

Figure I-5. Scenarios of carbon emission from land-use change in the tropical forests. Source: Houghton, 2005 modified by Quegan et al., 2012. 


\section{I.5.1.2 Monitoring of forest resources}

Monitoring and evaluation of a country's forest resources are carried out through National Forest Inventories (NFI), starting in the Nordic European countries in 1910 and continued with other European countries in the 1960s (Democratic Republic of Germany, France, Austria, and Spain) (Lund, 2009; Tomppo et al., 2010). The goals and objectives of NFI have changed according to a demand for information that users have made over time, such as timber production, forest biodiversity, the sustainability of forest resources, biomass storage, carbon capture, among others (Kleinn, 2017; Lund, 2009). This has led to an evolution of the NFI, initially, monitoring carried out by forest experts to assess the state of forest resources, and now monitoring carried out by multidisciplinary teams to meet commitments of global interest, resulting in an instrument for decision-making not only around forest resources (Kleinn, 2017). The NFIs have also evolved in other aspects such as the standardization of inventories carried out in the same country to be able to compare the results, and harmonization, which refers to the standardization between NFI of different countries (Alberdi et al., 2016; Tomppo et al., 2010). As an example of harmonization and since the NFI data are used to estimate the AGB and Carbon stored in the forest, IPCC guidelines have been incorporated to know the estimation uncertainty (IPCC, 2006) and MRV processes have also been incorporated to comply with the transparency in the estimation required by REDD+ ${ }^{1}$ mechanisms (Kleinn, 2017).

Since 1946, the Food and Agriculture Organization of the United Nations (FAO) has coordinated the generation of global forest data by compiling available national data. These reports had been published all 10 years and because of the rapid developments and increasing demand in global forest data the interval had been set for 5 years from 2005 onward. The world forest inventories, as they were called in the beginning, are now the FAO Forest Resources Assessments FAO-FRA (Garzuglia, 2018). These statistics since 2005 included AGB and carbon estimates under the IPCC specifications (FAO, 2006). In GFRA 2015, an improvement was implemented in the methodology applied to the evaluation called Long-term Strategy, which includes the improvement of the reports, the quality and the reliability of the data provided by the participating countries (Garzuglia, 2018).

IPCC and GFRA reports provide information on forest area and $\mathrm{CO}_{2}$ emissions due to forestry activities, as well as the dynamics of vegetation cover in cooperating countries. Both reports do emphasize the need to define and describe methodologies clearly and transparently and demand that the uncertainties associated with the results be explicitly reported for all target variables.

\section{I.5.2 National level: the example of Mexico}

\section{I.5.2.1 Policies and commitments}

The Government of Mexico has recognized the problems are caused by loss and degradation of forests and their relationship to climate change at national and international levels. Mexico signed its commitment to the UNFCCC in 1992, the Kyoto Protocol was signed at the COP in 1997, and in 2016 added to the Paris agreement (Chamber of Deputies, 2019; Morfín Ríos et al., 2015). Table I-1 shows the instruments and policies developed in Mexico to tackle the climate change effects and

\footnotetext{
${ }^{1}$ Reducing Emissions from Deforestation and forest Degradation and the role of conservation, sustainable management of forests and enhancement of forest carbon stocks in developing countries (REDD+)
} 
thus implement the commitments acquired at the international level. In addition, the economy of climate change effects has also been studied by the Mexican Government (SEMARNAT \& SHCP, 2009) and, together with the National Climate Change Strategy, the Government decided to conduct a sustainable, low-carbon economy based on the assessment of GHG emissions at the national level and by economic sectors (CICC, 2013; Mexican Government, 2019).

The National Forestry Commission in Mexico (CONAFOR), with the project "Reinforcing REDD+ and South-South Cooperation" (Mexico-Norway), developed the guidelines for the methodology to be used in Mexico for the GHG inventory (CONAFOR, 2015), emphasizing the challenge in AGB estimation of forest ecosystems. These guidelines were based on the IPCC proposal, that clearly establishes the use of AGB and carbon data from the Mexican National Forest Inventory (MNFI) for the estimation of emission factors in the sector LULUCF (Land-Use, Land-Use Change, and Forestry) (IPCC, 2006). This AGB and the carbon estimate should include its uncertainty estimation, as established by the REDD+ strategy in Mexico (CONAFOR, 2017b).

\begin{tabular}{|c|c|c|c|}
\hline $\begin{array}{l}\text { Instrument and / } \\
\text { or policies }\end{array}$ & Year* & Status & $\begin{array}{l}\text { Objective / chapter / clause related to climate } \\
\text { change }\end{array}$ \\
\hline $\begin{array}{l}\text { General Law of } \\
\text { Sustainable Forestry } \\
\text { Development }\end{array}$ & 2003 & $\begin{array}{l}\text { Updated in } 2018 \\
\text { (before } 2003, \\
\text { Forestry Law) }\end{array}$ & $\begin{array}{l}\text { It states that the MNFI is an instrument of national } \\
\text { policy and its estimates are used to develop programs } \\
\text { and strategies for adaptation and mitigation of } \\
\text { climate change (Chamber of Deputies, 2018a). }\end{array}$ \\
\hline $\begin{array}{l}\text { General Law on } \\
\text { Climate Change }\end{array}$ & 2012 & Updated in 2018 & $\begin{array}{l}\text { The chapters II and III regulate the implementation of } \\
\text { mechanisms for climate change adaptation and } \\
\text { mitigation (MRV systems) (Chamber of Deputies, } \\
\text { 2018b). }\end{array}$ \\
\hline $\begin{array}{l}\text { National Climate } \\
\text { Change Strategy }\end{array}$ & 2013 & Updated in 2019 & $\begin{array}{l}\text { National planning instrument. Proposal of actions to } \\
\text { be implemented in the medium and long term to face } \\
\text { the effects of climate change (Chamber of Deputies, } \\
\text { 2019). }\end{array}$ \\
\hline $\begin{array}{l}\text { National } \\
\text { Development Plan } \\
\text { of Mexico }\end{array}$ & 2013 & $\begin{array}{l}\text { Updated for the } \\
\text { period 2019- } \\
2024\end{array}$ & $\begin{array}{l}\text { Encourage economic development that will promote } \\
\text { the reduction of emissions of GHGs and adaptation to } \\
\text { climate change to improve the quality of life for the } \\
\text { population (Mexican Government, 2019). }\end{array}$ \\
\hline
\end{tabular}

*Year of publication or year when climate change regulations were included.

Table I-1. Instruments and policies to implement actions for adaptation and mitigation of climate change in Mexico.

\section{I.5.2.2 Further developing the National Forest Inventory}

The forest inventory in Mexico required a major improvement because the methodologies applied in the four national inventories, implemented from 1960 to 2001, were incompatible with each other (with respect to the set of variables, the sampling design, and the reference dates) and were therefore not immediately comparable (CONAFOR, 2012c). In 2002, a new inventory attempted to tackle the problem of inconsistency of protocols by harmonizing with US and Canadian NFI procedures, while promoting international cooperation with NAFC ${ }^{2}$ (SEMARNAT, 2004). The

\footnotetext{
${ }^{2}$ North American Forest Commission (NAFC) for Food and Agriculture Organization of the United Nations (FAO)
} 
primary objective of the MNFI was to support national sustainable forest development (SEMARNAT, 2004), by improving the estimation of biomass and carbon stocks and conserve the ecosystem quality (CONAFOR, 2012c). The MNFI in a more structured way started in the period of 2004-2009 and the first re-measurement 2009-2014. (CONAFOR, 2017a). The results of this inventory have been used to report forest biomass and carbon stocks to FRA since 2010 (CONAFOR, 2012c). Recently, MRV procedures have been incorporated to standardize the national carbon estimation process through the National REDD+ Strategy in Mexico (CONAFOR, 2017b; SEMARNAT \& INECC, 2017).

For the Mexican government, the importance of the forest has become evident through the policies generated over the last two decades around natural resources. These policies provide a framework for the development of this thesis topic that describes the methodologies for the calculation and reporting of uncertainties in the AGB estimation. The research in this study complements the information reported by CONAFOR (CONAFOR, 2014a), since non-sampling errors are not reported. The information in this thesis is the first study reporting measurement and prediction errors along with sampling errors for the MNFI in Durango.

\section{I.6 Outline of the dissertation}

The thesis work includes the following topics as illustrated in Figure I-6:

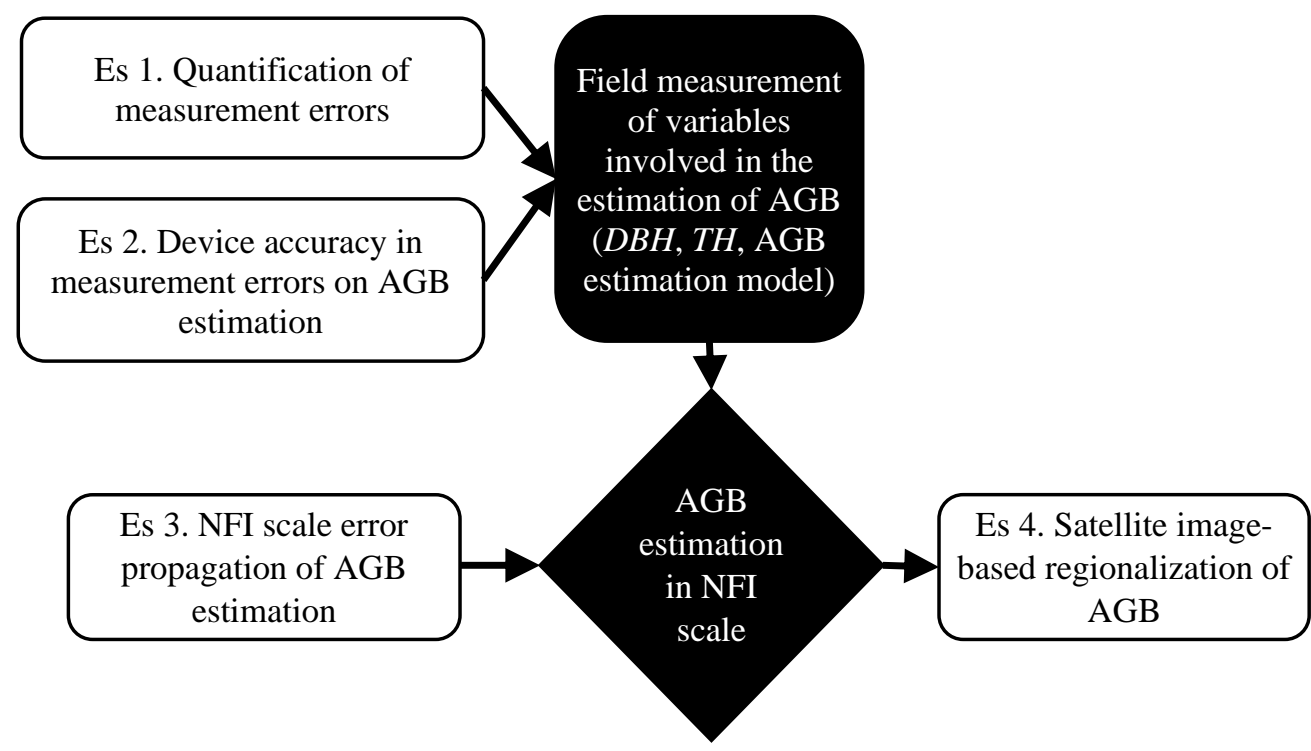

Figure I-6. Structure of the dissertation.

Empirical study 1. Quantification of measurement errors: There is no evidence of published works where the measurement error was estimated in dasometric variables for temperate forests of Durango and in general in the context of the Mexican NFI. Studies in other countries have shown that applying double measurement allows knowing the discrepancy of measurements and can describe the measurement error. For this reason, double measurements were carried out for $D B H$ and $T H$ in pine forest in El Salto, Durango, Mexico to quantify the measurement errors occurring during fieldwork for $\mathrm{DBH}$ and $\mathrm{TH}$. 
Empirical study 2. Device accuracy in measurement errors on AGB estimation: To determine the uncertainty of the best estimate, in $D B H$ and $T H$ measurements, a case study was developed to compare two devices of different precision for each variable and the effect that this difference generates in the accuracy of the AGB estimation and error propagation.

Empirical study 3. NFI scale error propagation of AGB estimation: The AGB of the temperate forest was estimated for the whole state of Durango, Mexico, using the data of the MNFI and allometric models to calculate de AGB. The uncertainty propagation in AGB was estimated with the $M C M$, incorporating the distributions of the errors from the sources of uncertainty: fieldwork measurements (Topic 1), allometric models and sampling design.

Empirical study 4. Satellite image-based regionalization of AGB: AGB was modeled with remote sensing using Landsat images and MNFI fieldwork as the reference data of AGB for the state of Durango, Mexico.

\section{I.7 Objective and research questions}

\section{I.7.1 General objective}

The overall objective is to contribute to a better understanding of the role of errors and error propagation in AGB estimates for the state of Durango, from data of the Mexican NFI, which in turn will support carbon reporting at the national scale and helps make the NFI results more meaningful and better interpretable.

From this overall objective, the following technical research questions are being derived all oriented towards contributing to achieving the overall objective:

\section{I.7.2 Research questions}

Empirical study 1

i. Which Probability Density Function (PDF) describes best the measurement errors in $D B H$ and $T H$ for pine forest?

ii. How much are the contributions of the measurement errors and allometric errors in comparison with the sampling error, in AGB estimation?

iii. Which differences exist in the error propagation results from GUM Method in comparison with Monte-Carlo simulation Method?

Empirical study 2

i. How does the PDF of measurement error changes with different measuring equipment for $D B H$ and $T H$ ?

ii. How does the measurement error in $\mathrm{DBH}$ and $\mathrm{TH}$ contribute to the total uncertainty in AGB estimation, when the measurement error comes from devices of different precision?

iii. How is the relationship between non-sampling errors and sampling errors, when the sample size increases?

Empirical study 3 
i. Are measurement, prediction, and sampling, the only sources of uncertainty associated with the error propagation in a scale of NFI?

ii. What are the contributions of the different sources of error in the estimation of AGB to the temperate forest in Durango State, using MCM?

iii. How does the sample size affect the total uncertainty, when the analysis is made into the strata of the temperate forest?

Empirical study 4

i. Is the MNFI data suitable as a source of fieldwork information to predict AGB with satellite information from the Landsat sensor?

ii. Which are the response variables from the Landsat sensor, that best model the AGB for Durango temperate forest and substrata in the temperate forest?

iii. Are goodness-of-fit parameters improved in the estimation of AGB with Landsat when carrying out stratification with vegetation types? 



\section{Chapter II: Materials}

This research has been implemented through four studies developed with information on the Mexican National Forest Inventory in the state of Durango. However, it was necessary to perform two experiments in El Salto, Durango, Mexico and Göttingen, Germany, to improve understanding and analysis of the topic under study.

\section{II.1 Study Area}

\section{II.1.1 Durango State, Mexico}

The main study area in this thesis work was the state of Durango in Mexico (third and fourth empirical studies). This state lies between the coordinates (X, Y): corner North West (277055, 2968491) and corner South East (755631, 2472422) according to the UTM projection, datum WGS84 and 13N zone (Figure II-1). Durango has an area of $123450 \mathrm{~km}^{2}$. It is the fourth largest state and represents $6.3 \%$ of the national area in Mexico. It is divided into 39 municipalities and had a population as of 2015 of almost 1.8 million inhabitants (INEGI, 2016).

The topography of Durango is rugged, the altitude difference ranges from 440 meters above sea level (masl) in the Piaxtla river bed, southeast of the state, up to 3328 masl in the Gordo hill, in the south of the state. The physiography of the state has four provinces: the mountain chain Sierra Madre Occidental (SMO) from the southwest to the center (71\% of Durango), Northern mountains and plains in the north-central area (15\%), to the east lies Part of the Mesa del Centro (9\%) and to the northeast the mountain chain Sierra Madre Oriental (5\%) (CONABIO, 2017).

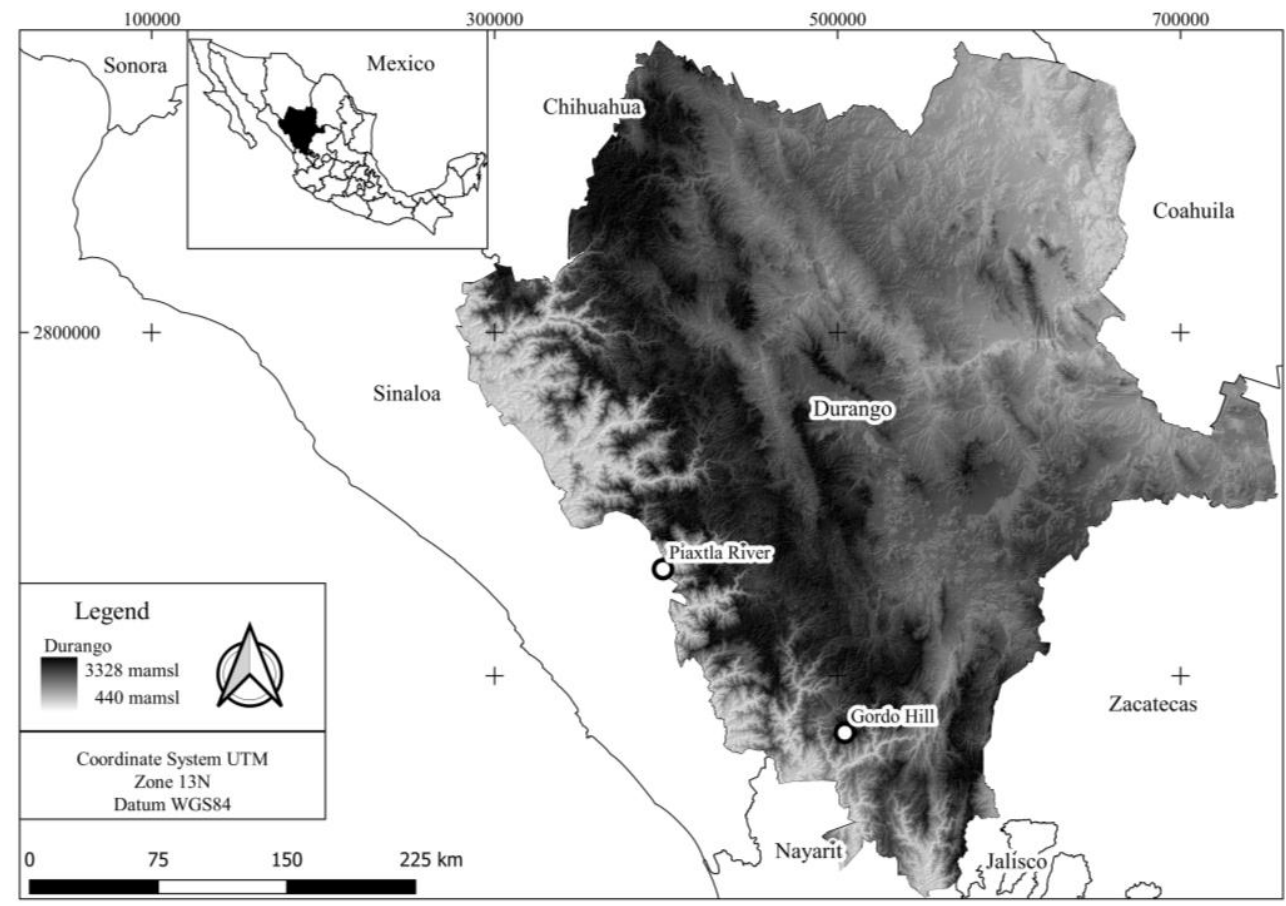

Figure II-1. Durango State in the context of Mexico. 
The main geological formation is an extrusive igneous with $58 \%$ of the state, followed by sedimentary material (23\%), soil formations $(16 \%)$, and the rest is a metamorphic rock and intrusive igneous rock (3\%). Soil types distribution is uneven in the state, the Leptosol type is present in $32 \%$ of the territory, Phaeozem in $15 \%$ and Luvisol in $13 \%$, these are the main soils with more than half of the surface of the state. The predominant climate in $54 \%$ of the area ranges from very dry to semi-dry, in second place of importance is the temperate climate covering 34\% of Durango, placed principally on the area of the mountain chain SMO (INEGI, 2016; CONABIO, 2017).

Anthropogenic activities, in Durango, cover 30\% of the surface (3.7 million ha) and vegetation cover the remaining $70 \%$ (8.6 Mha). Timber forest, which includes temperate forest and tropical forest, is $47 \%$ (5.8 Mha) of the state. Conifer forest is the largest area of vegetation with about 19\% (2.3 Mha). The non-timber forest, including shrubs and desert vegetation, is $22.8 \%$ of the state of Durango with 2.8 Mha. (INEGI, 2017).

\section{II.1.1.1 El Salto, Durango}

The study area, that was used to do the first empirical study of measurement errors in $D B H$ and $T H$, is in the temperate forest of southwest Durango, Mexico. Including areas of high-density pine plantations of 20-40 years old without a management plan. The country is subdivided into Forest Management Units (UMAFOR in Spanish), according to the National Forest Commission (CONAFOR), the institution leading the management of forest resources in Mexico. The fieldwork was made in the UMAFOR 1008 or "El Salto" characterized by mixed and uneven-aged temperate forest stands (Figure II-2). The UMAFOR 1008 has 558,270 ha of surface, the 52\% of the area is occupied with temperate forest, and this is equal to $7 \%$ of the area of temperate forest in the state of Durango.

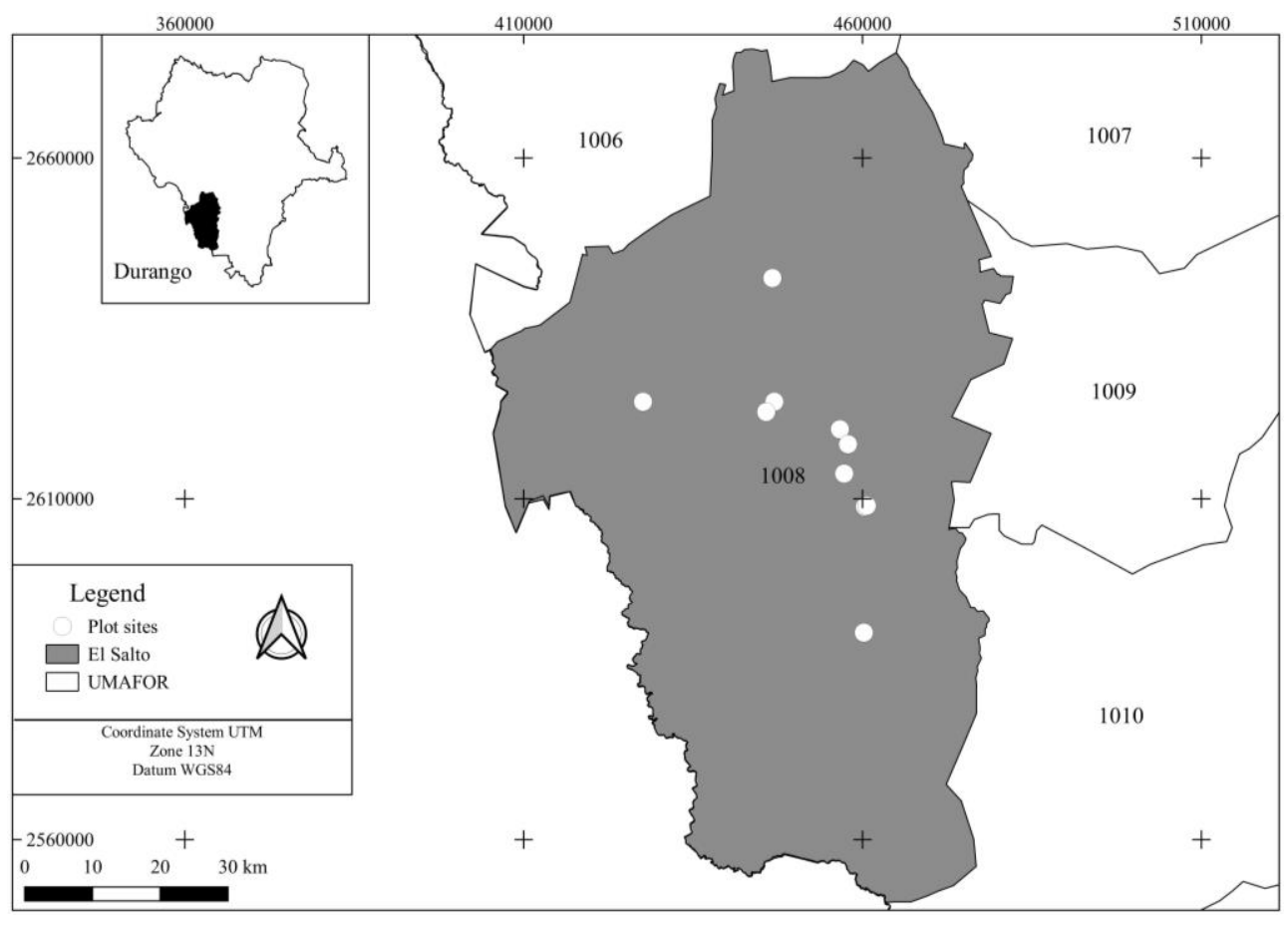

Figure II-2. Location map of plot sites and UMAFOR 1008 in the State of Durango. 
With an altitude of 400 - 3262 masl; the predominant climate is temperate, sub-humid with rain during summer (SEMARNAT, 2014). The average annual temperature of $10.7^{\circ} \mathrm{C}$ and an average annual rainfall of 760 - $1490 \mathrm{~mm}$ during the months of June to September. This management unit is located in the mountain chain SMO. The forest vegetation is principally a mixture of the genus Pinus (P. engelmannii, $P$. durangensis, $P$. chihuahuana, P. cooperi, $P$. leiophylla, $P$. teocote, $P$. arizonica, $P$. lumholtzii), genus Quercus ( $Q$. sideroxyla, $Q$. rugose, $Q$. fulva, $Q$. mcvaughii and $Q$. scytophylla), and shrub species of the genus Juniperus sp., Arbutus sp. and Alnus sp. among others (González-Elizondo et al. , 2012).

\section{II.1.2 Göttingen, Germany}

The second empirical study, comparing the measurement uncertainty committed in fieldwork using different devices took place in the State forest in Northern of Göttingen, Lower Saxony, Germany (Figure II-3). This forest is located at 54 34' North latitude $-9^{\circ} 57^{\prime} 40^{\prime \prime}$ 'East longitude and a maximum altitude of 427.5 masl (Nagel \& Wunderlich, 1976). The annual rainfall in Göttingen, $628 \mathrm{~mm}$, is uniformly distributed over the year and temperature ranges from 1 to $17.4{ }^{\circ} \mathrm{C}$, obtaining with the average information of 1971-2000 (GAUG, 2017).

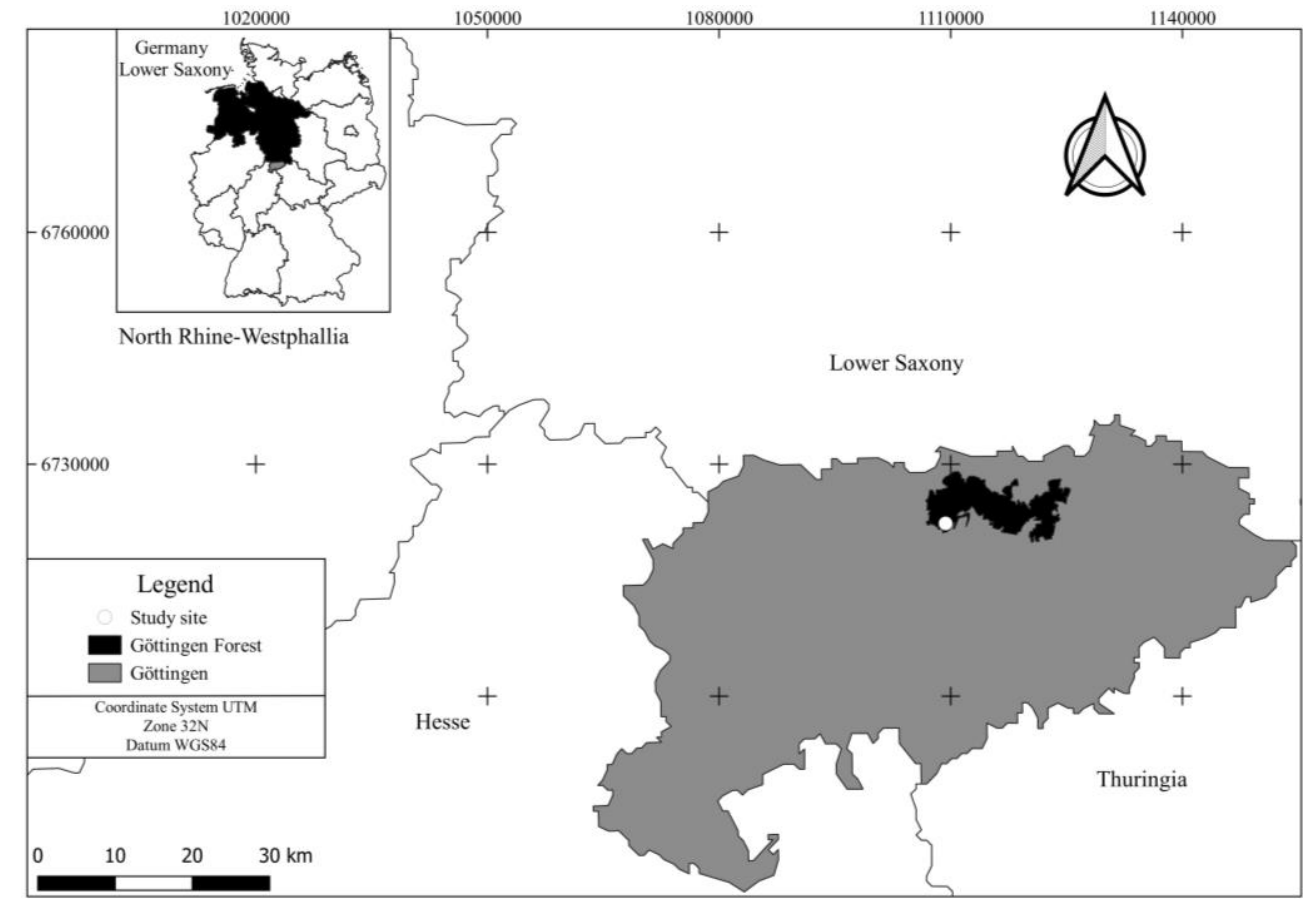

Figure II-3. Location map of the study area in the Göttingen Forest, Germany.

The studied forest tract belongs to the Highland of southern Lower Saxony and specifically to the "Forest of Göttingen" (Brumme \& Khanna, 2009), 4 km north-east of Göttingen city center. The principal forest species in the study site are beech (Fagus sylvatica) as a dominant species, mixed with European ash (Fraxinus excelsior), field maple (Acer campestre), Norway spruce (Picea abies), wild cherry (Prunus avium), and few other species. 


\section{II.2 Material}

\section{II.2.1 National Forest Inventory information}

For the third empirical study, ground measurements were provided by CONAFOR (Table II-1). The database available contains the field data of the first MNFI in the state of Durango (Figure II-4) in the period 2004-2009 (CONAFOR, 2012c), as well as the data of the first re-measurement of the MNFI 2009-2014 (CONAFOR, 2017a). The data acquisition in the fieldwork was based on the "Manual and procedures for field sampling" made by CONAFOR (CONAFOR, 2009b). The geographic location associated with the field information was the center of the Cluster or Primary Sampling Unit (PSU) and the center of each subplot or Secondary Sampling Units ( $S S U$ ), the location of the tree was not recorded (CONAFOR, 2012c). The geographic information was recorded with a conventional GPS receiver in projection UTM13N, CRS ITRF92, Datum ITRF92, ellipsoid GRS1980 and units in meters (CONAFOR, 2009b). Geographic information was recorded with an accuracy of less than or equal to 15 meters (CONAFOR, 2009b).

\begin{tabular}{ccccc}
\hline MNFI dataset & Tree No. & Cluster No. & Subplots No. & Species \\
\hline $2004-2009$ & 118647 & 1826 & 6170 & 220 \\
$2009-2014$ & 118641 & 1822 & 6090 & 277 \\
\hline
\end{tabular}

Table II-1. General information in sampling units of MNFI in Durango, Mexico.
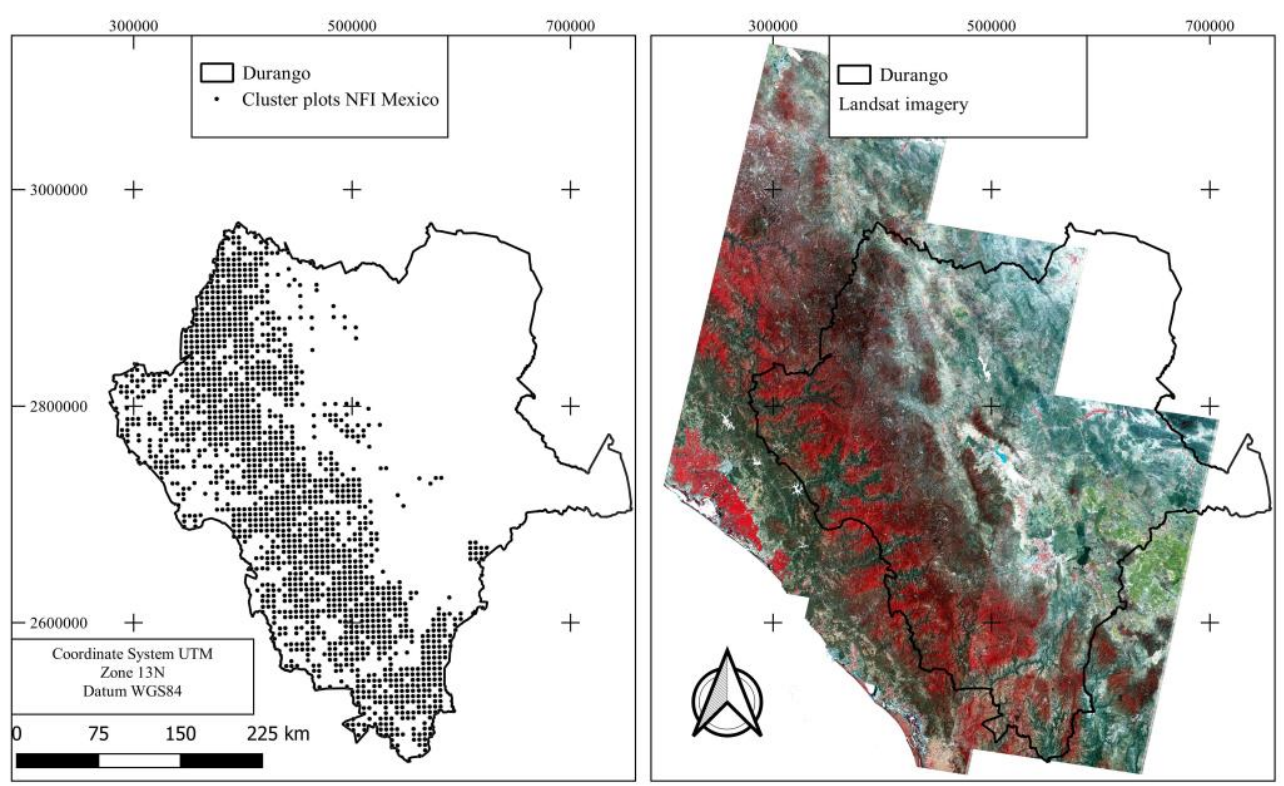

Figure II-4. Left: Grid of Primary sampling units (PSU) of the MNFI for the temperate forest in Durango. Right: Landsat 5 mosaic imagery false-color composite with RGB: 432 combination to enhance vegetation in red color, covering all forested lands in Durango.

\section{II.2.2 Satellite imagery information}

To estimate the AGB in the third empirical study from remote sensing data, Landsat imagery was used (Landsat 5 Thematic Mapper TM and Landsat 8 Operational Land Imager OLI) with a spatial resolution of $30 \mathrm{~m}$ (USGS, 2015). The images were downloaded from 
https://earthexplorer.usgs.gov/ . The images required were the product with high-level surface reflectance (Tier 1) with $R M S E \leq 12 \mathrm{~m}$ of geometric verification (Young et al., 2017).

For the third empirical study, two field data sets were used: MNFI 2004-2009 and MNFI remeasurement 2009-2014. For these two time periods, in the fourth empirical study satellite imagery was required. The selected scenes are listed in Table II-2 and they came from the last year of each field campaign in MNFI in 2007 (Landsat 5 TM) and in 2013 (Landsat 8 OLI). With these data was developed the model-based assessment.

\begin{tabular}{ccccc}
\hline \multirow{2}{*}{ Path/row } & \multicolumn{2}{c}{2007} & \multicolumn{2}{c}{$\mathbf{2 0 1 3}$} \\
\cline { 2 - 5 } & $\begin{array}{l}\text { Landsat 5 TM } \\
\text { (day.month) }\end{array}$ & $\boldsymbol{R M S E}$ (m) & $\begin{array}{l}\text { Landsat 8 OLI } \\
\text { (day.month) }\end{array}$ & $\boldsymbol{R M S E}(\mathbf{m})$ \\
\hline $32 / 41$ & 28.04 & 4.62 & 28.04 & 6.98 \\
$32 / 42$ & 28.04 & 4.46 & 28.04 & 5.90 \\
$32 / 43$ & 28.04 & 5.27 & 28.04 & 7.64 \\
$31 / 42$ & 21.04 & 4.23 & 23.05 & 7.81 \\
$31 / 43$ & 21.04 & 4.89 & 23.05 & 6.49 \\
$31 / 44$ & 21.04 & 4.77 & 23.05 & 8.01 \\
$30 / 43$ & 30.04 & 4.38 & 30.04 & 7.26 \\
$30 / 44$ & 30.04 & 4.48 & 30.04 & 8.09 \\
\hline
\end{tabular}

Table II-2. Satellite imagery scenes of Landsat used in this study to estimate AGB in Durango, Mexico. RMSE was expressed in meters and characterizes the result of the geometric correction with ground control points.

\section{II.2.3 Vegetation and land-use information}

Vegetation and land-use series (LUVS) is a national product developed through the interpretation of satellite imagery: Landsat and SPOT (CONAFOR, 2014b). This product started in 1993 and has been incorporating new tools to improve the quality of the information obtained over time and has been used for the analysis of the land-use and vegetation change nationwide in Mexico (INEGI, 2017). The LUVS, Series IV and Series V, were used to demarcate the area of the vegetation types found in the MNFI (CONAFOR, 2012c, 2017a).

The Figure II-5 shows the main vegetation types, the areas without vegetation and the anthropogenic uses, for the Series IV and V of INEGI in the state of Durango. The INEGI, includes in the anthropogenic uses the urban areas, agriculture, grassland, among others (INEGI, 2014). The vegetation strata, inside of the temperate forest in Durango, are shown in Figure II-6, also for the two LUVS. 


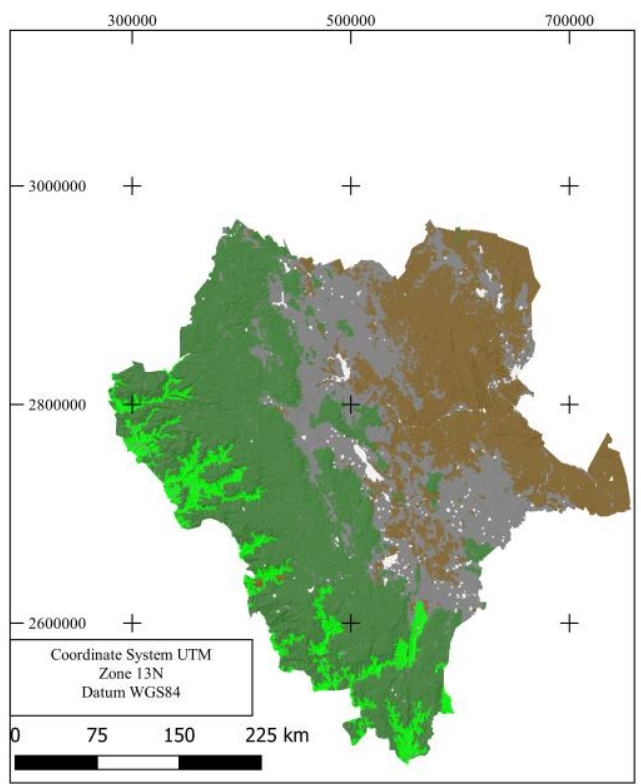

Series IV (2007-2008)

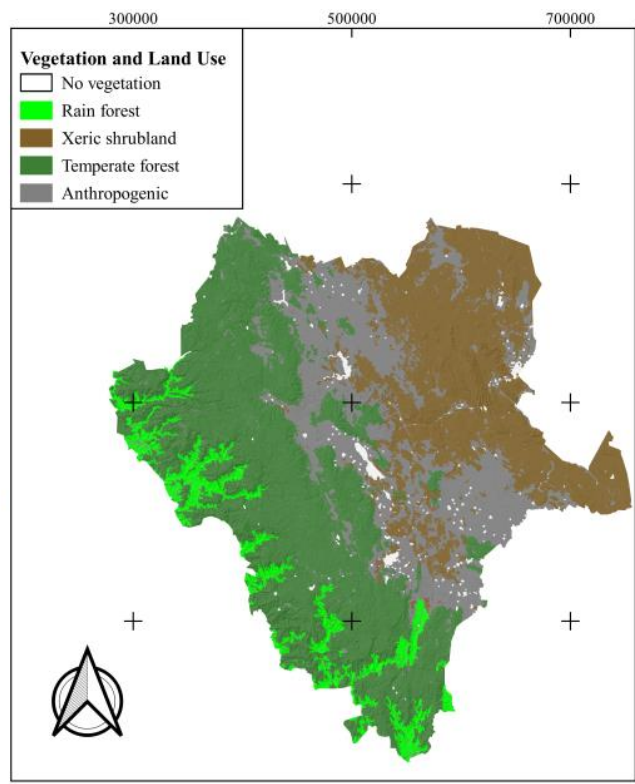

Series V (2012-2013)

Figure II-5. Vegetation and land-use limits of Durango for the LUVS of 2007-2008 (Serie IV) and 2012-2013 (Serie V).

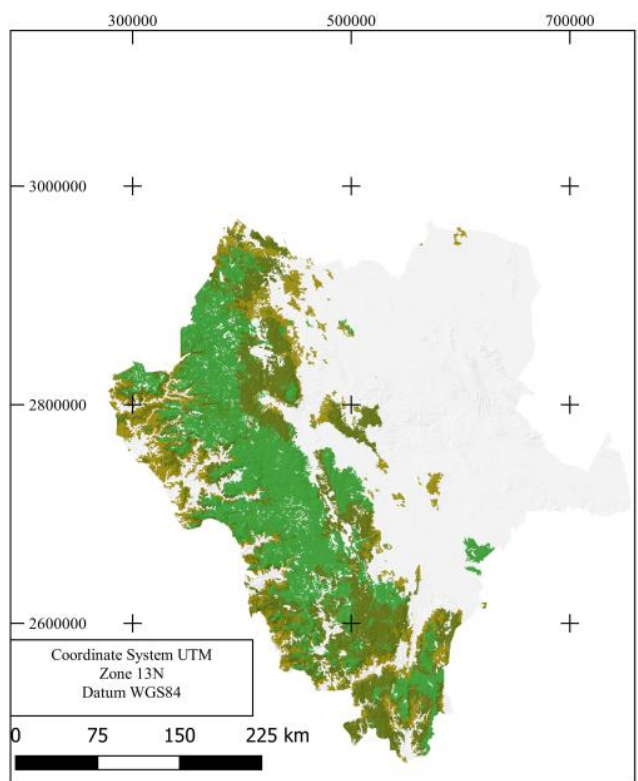

Series IV (2007-2008)

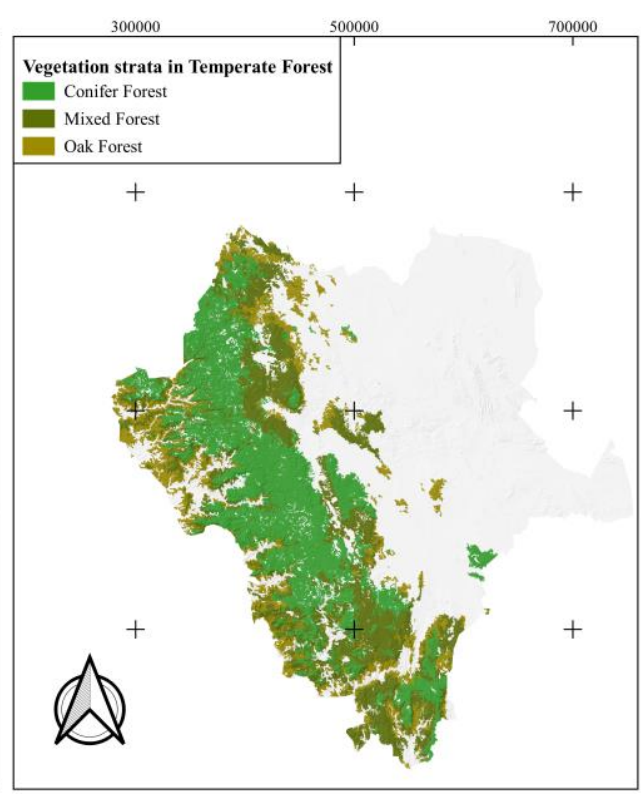

Series V (2012-2013)

Figure II-6. Vegetation strata limits of temperate forest in Durango according to vegetation Series IV and V.

In the third empirical study, using the MNFI data, AGB estimation for the temperate forest and the strata inside the temperate forest (conifer, mixed, and oak) were provided. Using the estimates per vegetation type and demarcated area in Figure II-6, in the fourth empirical study, the AGB stored in the state of Durango was estimated with a sampling-based assessment. 


\section{Chapter III: Methodologies}

\section{III.1 Empirical study 1: Double measurement of dasometric variables to estimate the} measurement uncertainty and error propagation in aboveground biomass estimation in pine forests

\section{III.1.1 Sampling design}

Plot locations were selected by a broader project ${ }^{3}$ based on stand competition conditions (tree density and age-class). The selection of the plot had two limiting reasons; therefore, it was necessary the prior knowledge provided by the forestry technicians of the region. The first reason was safety issues due to social problems; the second reason was the owner's permission to work in the sampling sites. After applying the above criteria on the sampling sites, 10 locations met the factors to be considered sampling sites (Figures II-2, III-1): La Victoria, La Campana, Borbollones, La Campana 2, Pueblo Nuevo, Pueblo Nuevo 2, Pueblo Nuevo 3, La Campana 3, El Brillante 2 y El Brillante 3.

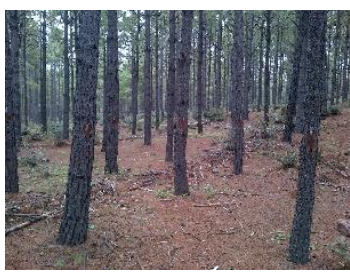

La Victoria

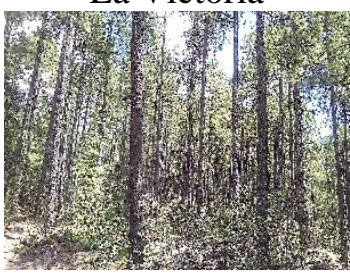

Pueblo Nuevo

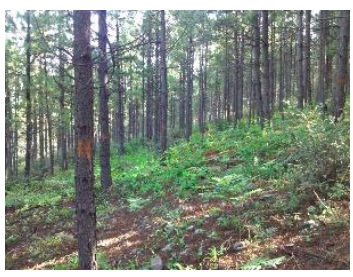

La Campana

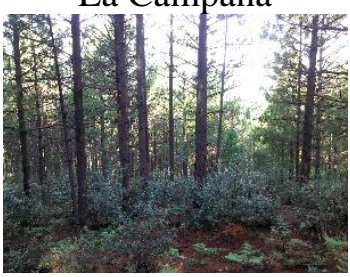

Pueblo Nuevo 2

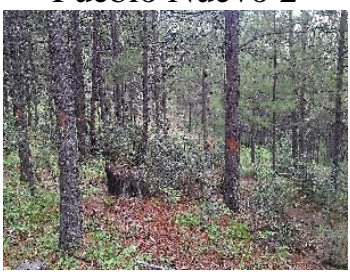

El Brillante 2

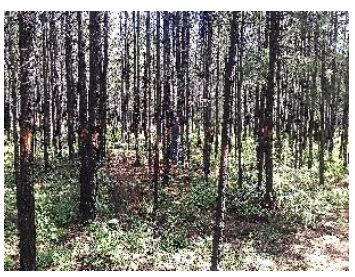

Borbollones

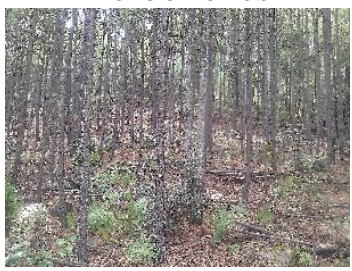

Pueblo Nuevo 3

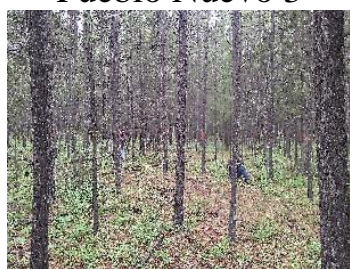

El Brillante 3

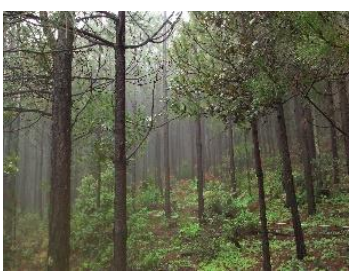

La Campana 2

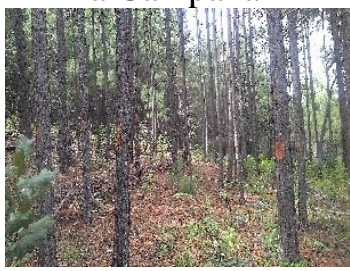

La Campana 3

Figure III-1. Pictures of the pine forest in El Salto, Durango, where the sampling sites were located.

\section{III.1.2 Plot design}

The aforementioned project determined the plot design and provided the field crew. My role was to coordinate the fieldwork and I was part of the observers of the dasometric variables. The plots were established in August and September of 2014. The plot size was 95 X 60 meters; subdivided into six

\footnotetext{
3 "Installation of a thinning experiment in forests of UMAFOR 1008" (Corral-Rivas et al, 2013)
} 
subplots of 25 X 25 meters with a corridor of ten meters between the plots (Figure III-2). The division into subplots was made for later thinning treatments by the project above mentioned; however, for this study, the data of the six subplots together was considered as a single plot data. The boundaries of the subplots were oriented North-South and East-West and cords were used to make the plot limits visible. The plots remained marked throughout the period of the measurements.

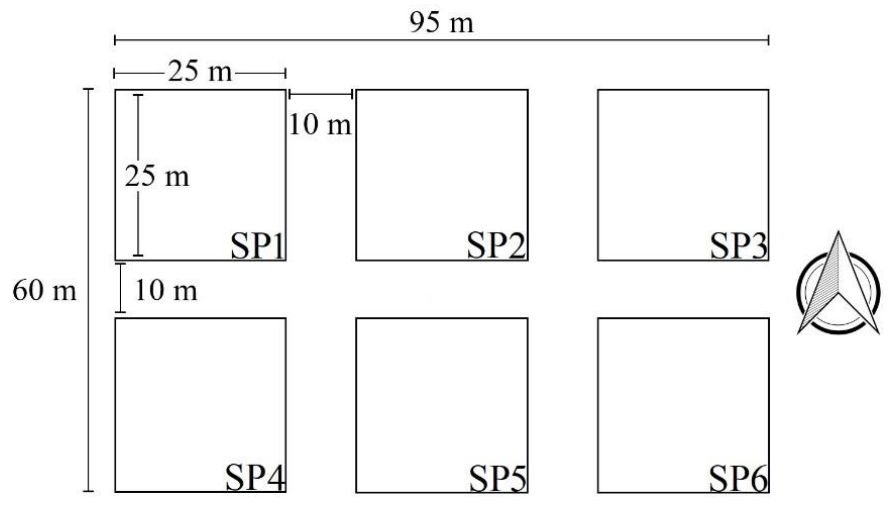

Figure III-2. Size and arrangement of the subplots.

The trees in the plots were marked and numbered with a metal plate and their position recorded with $\mathrm{x}-\mathrm{y}$ local coordinates for each subplot. These tree marks facilitated the identification of the sample trees, during the measurement. The metal plates were oriented to the center of the subplot for easier reference.

\section{III.1.3 Experiment design}

Field data collection followed the procedures defined in the "Manual and procedures for field sampling", a protocol developed by CONAFOR for the MNFI 2004-2009 (CONAFOR, 2012c). This document describes exhaustively how to do a standardized data collection of dendrometric measurements (i.e. $D B H, T H$, etc.) in MNFI.

Devices used in the measurement were calibrated regularly to avoid miss-calibrations. The center of each plot and subplot was recorded by a Sokkia CX total station with two base stations, which was also used to measure azimuth and distances from the center of the plot to the trees stem surface at breast height. Tree position was then the distance measured plus half the diameter of the tree. Positions measured were post-processed and was obtained a precision of $2 \mathrm{~mm}$ using data from base stations and projected in the UTM WGS84 system of zone $13 \mathrm{~N}$. DBH was measured in two directions, North-South and East-West, similar to the marking of the sub-plots and a Caliper with $\mathrm{mm}$ accuracy was used. The $T H$ was measured with a Vertex $I V$, this device had $0.1 \mathrm{~m}$ as the smallest unit of measurement. Species identification was done by support staff who had experience in the identification of regional trees.

A procedure of two "blind" measurements was used as recommended by Condit (1998) as a measure of quality assurance. This method consisted of the following steps: i ) Measuring a variable to all the trees in the plot, this was the first measurement made by one person, ii ) Repeatedmeasurement of the same variable for all trees in the plot without access to previous measurement made, being a measurement independently made by a second observer. In the first cycle, $D B H, T H$, 
tree location and species were recorded. In the second cycle, $D B H$ and $T H$ were recorded; because of the relatively low species diversity in the sample plots, a re-assessment of tree species was not done, assuming that errors due to species-misidentifications be non-existent. The re-measurement of most plots was done on the same day or the day after the first measurement. At one sampling site, it could only be done about one week later. The recording of field data was conducted by two different observers, one for point 1 in time and the second one for the re-measurement.

\section{III.1.4 Aboveground biomass (AGB) estimation}

The AGB estimation was calculated indirectly using allometric models. Field variables used in these models were $D B H, T H$ and tree species. Four species of pine were identified (Pinus cooperi, P. leiophylla, P. strobiformis and P. teocote), trees from genus Arbutus spp, Juniperus spp and Quercus spp, and one tree of Abies durangensis. A selection criterion for the model was developed because a pool of 46 models was found for the species identified and is represented in the following flow chart (Figure III-3).

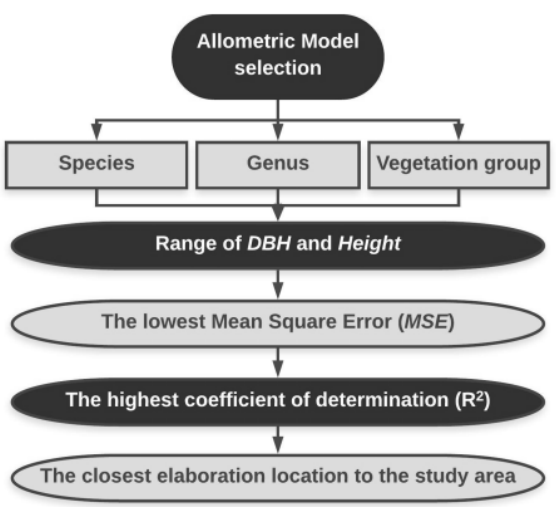

Figure III-3. Flow chart to select the allometric model for AGB estimation, adapted from CONAFOR (CONAFOR, 2014b).

The first step in the selection criteria was to identify the tree in the field at the sp/genus/vegetation group level. With the field identification, models that meet the first criterion were then compared with the valid range, which must not be exceeded by field data ( $D B H, T H$, crown diameter) to avoid a systematic error. The next two criteria were measures of the goodness of the model fit, selecting the models with the lowest $M S E$ and the highest determination coefficient $\left(\mathrm{R}^{2}\right)$. The last criterion was the selection of the model that has been adjusted with trees in the study area or the model that was adjusted close to the study area. Even though not included in the criteria, the selected model used more than 30 trees to meet the requirement of a biomass estimate in a homogeneous and species-specific stand, as referred to Picard et al. (2012).

As before mentioned, in "El Salto" five genera and in the genus Pinus spp four species were identified, therefore, models made at species/genus levels were used. Rojas-García et al. (2015a) made a compilation of 478 allometric models to estimate AGB in Mexico; this study reported in Durango 27 and three AGB models for the genus Pinus spp and Quercus spp, respectively. In 2017, Vargas-Larreta et al. published a study conducted in the Durango temperate forest where they obtained allometric models of AGB for species-specific and genus of the main commercial species. 
Allometric models, adjusted by Vargas-Larreta et al. (2017), satisfied the selection criteria and are shown in Table III-1. The genus Abies spp in the state of Durango is infrequent (Quiñones-Pérez et al., 2012) and no studies on AGB estimation have been conducted, for this reason, the allometric model proposed by Avendaño et al. (2009) adjusted for the Abies religiosa species was used.

\begin{tabular}{|c|c|c|c|c|c|c|c|c|c|c|c|}
\hline$s p$ & $\begin{array}{c}A G B \\
\text { component }\end{array}$ & $\begin{array}{l}\ln \\
\alpha\end{array}$ & $\begin{array}{l}\boldsymbol{\beta}_{1} \ln \\
D B H\end{array}$ & $\begin{array}{c}\boldsymbol{\beta}_{2} \ln \\
T H \\
\end{array}$ & $\begin{array}{c}\boldsymbol{\beta}_{3} \\
D B H^{2} T H \\
\end{array}$ & $R^{2}$ & $\begin{array}{c}\text { RMSE } \\
\mathrm{kg} \\
\end{array}$ & $n$ & $\begin{array}{l}\mathrm{DBH} \\
(\mathrm{cm}) \\
\end{array}$ & $\begin{array}{c}\mathrm{TH} \\
(\mathrm{cm}) \\
\end{array}$ & $\begin{array}{l}\text { Tree } \\
\text { No. }\end{array}$ \\
\hline $\begin{array}{l}\text { Abies } \\
\text { duranguensis }^{1}\end{array}$ & Total & 1.074 & 2.510 & & & 0.99 & & 26 & $6.5-79.0$ & $6.8-42.4$ & 1 \\
\hline \multirow{5}{*}{$\begin{array}{l}\text { Arbutus } \\
\text { spp }^{2}\end{array}$} & stem & 1.067 & 1.664 & 0.865 & & 0.93 & 15.11 & & & & \\
\hline & bark & 1.003 & 1.518 & 0.950 & & 0.87 & 0.85 & & & & \\
\hline & branches & 1.021 & 1.812 & 0.819 & & 0.95 & 6.99 & & & & \\
\hline & foliage & 1.007 & 1.503 & 0.848 & & 0.79 & 1.72 & & & & \\
\hline & & Total $(\Sigma$ & & & & 0.95 & 22.81 & 49 & 7.9-44.8 & $2.4-25.0$ & 4 \\
\hline \multirow{5}{*}{$\begin{array}{l}\text { Juniperus } \\
\text { spp }^{2}\end{array}$} & stem & & & & 0.013 & 0.91 & 28.98 & & & & \\
\hline & bark & & & & 0.001 & 0.57 & 3.15 & & & & \\
\hline & branches & & & & 0.002 & 0.81 & 5.87 & & & & \\
\hline & foliage & & & & 0.001 & 0.67 & 3.92 & & & & \\
\hline & & Total $(\Sigma$ & & & & 0.90 & 37.00 & 48 & $10.0-43.7$ & $4.5-21.5$ & 23 \\
\hline \multirow{5}{*}{$\begin{array}{l}\text { Pinus } \\
\text { cooperi }^{2}\end{array}$} & stem & 1.032 & 2.093 & 0.769 & & 0.97 & 41.22 & & & & \\
\hline & bark & 1.011 & 1.676 & 0.746 & & 0.81 & 10.43 & & & & \\
\hline & branches & 1.008 & 1.599 & 1.347 & & 0.89 & 27.06 & & & & \\
\hline & foliage & 1.051 & 1.223 & 0.600 & & 0.74 & 3.79 & & & & \\
\hline & & Total $(\Sigma$ & & & & 0.94 & 87.21 & 103 & $5.5-52.3$ & $4.2-28.0$ & 3956 \\
\hline \multirow{5}{*}{$\begin{array}{l}P . \\
\text { leiophylla }\end{array}$} & stem & & & & 0.016 & 0.94 & 68.35 & & & & \\
\hline & bark & & & & 0.001 & 0.89 & 6.92 & & & & \\
\hline & branches & & & & 0.007 & 0.63 & 67.38 & & & & \\
\hline & foliage & & & & 0.0003 & 0.81 & 2.53 & & & & \\
\hline & & Total $(\Sigma$ & & & & 0.92 & 110.21 & 84 & $8.4-55.3$ & $5.4-29.2$ & 79 \\
\hline \multirow{5}{*}{$\begin{array}{l}P . \\
\text { strobiformis }{ }^{2}\end{array}$} & stem & 1.007 & 2.022 & 1.309 & & 0.90 & 88.01 & & & & \\
\hline & bark & 1.031 & 1.100 & 1.099 & & 0.88 & 6.34 & & & & \\
\hline & branches & 1.016 & 1.906 & 0.701 & & 0.93 & 15.24 & & & & \\
\hline & foliage & 1.040 & 1.535 & 0.318 & & 0.72 & 5.55 & & & & \\
\hline & & Total $(\Sigma$ & & & & 0.93 & 93.02 & 98 & $5.0-49.0$ & $6.3-26.6$ & 32 \\
\hline \multirow{5}{*}{$\begin{array}{l}P . \\
\text { teocote }^{2}\end{array}$} & stem & 1.045 & 1.895 & 0.847 & & 0.92 & 58.50 & & & & \\
\hline & bark & 1.003 & 1.962 & 0.662 & & 0.93 & 9.34 & & & & \\
\hline & branches & 1.005 & 1.788 & 1.123 & & 0.93 & 3.02 & & & & \\
\hline & foliage & 1.031 & 1.784 & & & 0.73 & 16.67 & & & & \\
\hline & & Total $(\Sigma$ & & & & 0.95 & 76.11 & 81 & $10.0-45.0$ & $4.5-24.7$ & 124 \\
\hline \multirow{5}{*}{$\underset{s p p^{2}}{\text { Quercus }}$} & stem & 1.123 & 1.774 & 0.687 & & 0.78 & 94.25 & & & & \\
\hline & bark & 1.008 & 0.008 & & & 0.65 & 27.68 & & & & \\
\hline & branches & 1.051 & 0.050 & 0.342 & & 0.57 & 53.64 & & & & \\
\hline & foliage & 1.086 & 0.082 & & & 0.29 & 11.95 & & & & \\
\hline & & Total $(\Sigma$ & & & & 0.82 & 134.12 & 423 & $7.0-57.0$ & $3.3-24.8$ & 43 \\
\hline
\end{tabular}

AGB component $\left(\mathrm{kg} \mathrm{ha}^{-1}\right)=W_{i}=\alpha_{i} X_{j}^{\beta_{i j}}+\varepsilon_{i}$; AGB Total $\left(\mathrm{kg} \mathrm{ha}^{-1}\right)=W_{t}=\sum_{i=1}^{n} W_{i}+\varepsilon_{t}$; where $X_{j}=$ tree variables, $\alpha_{i}$ and $\beta_{i}=$ coefficients estimated, $\varepsilon_{i}$ and $\varepsilon_{t}=$ error terms; $R^{2}=$ coefficient of determination; $R M S E=$ root mean square error; $n=$ number of trees used to fit the model; $D B H(\mathrm{~cm})=D B H$ range of trees used to fit the model; $T H(\mathrm{~cm})=T H$ range of trees used to fit the model; Tree No.=number of trees registered in El Salto, Durango.

${ }^{1}$ (Avendaño Hernandez et al., 2009)

2 (Vargas-Larreta et al., 2017)

Table III-1. Allometric models used in the pine forest of El Salto, Durango to estimate AGB based in $\mathrm{DBH}$ and $\mathrm{TH}$ measurements. 


\section{III.1.5 Quantifying uncertainty}

\section{III.1.5.1 Source of uncertainties}

\section{III.1.5.1.1 Methods to estimate measurement error at tree-level}

$D B H$ and $T H$ were measured for 4262 trees. The measurement $(M 1)$ and the re-measurement $(M 2)$ of the variables were made independently and each was considered as a trial mensuration. Therefore, the difference of the M1-M2 was treated as a discrepancy (Taylor, 1997).

The relationship between discrepancies in $D B H$ measurement and the $D B H$ size is shown in Figure III-4. The dispersion chart shows a concentration of the point cloud close to zero, and in trees with $D B H$ less than $30 \mathrm{~cm}$. In the lower part of the figure, the dispersion chart of $T H$ discrepancies by $T H$ tree size is shown. Most of the values in $T H$ are also concentrated close to zero and in trees larger than 5 meters but no more than $20 \mathrm{~m}$ of $T H$. To verify the concentration of data close to zero was applied a Kurtosis Test, and the discrepancies distributions were classified as leptokurtic (Kurt ${ }_{D B H}=8.030$, Kurt $_{T H}=4.394$ ). Using the Lilliefors Test, normal distribution was rejected for both variables discrepancies $\left(\mathrm{p}_{D B H}<2.2 \mathrm{e}^{-16}, \mathrm{p}_{T H}<2.2 \mathrm{e}^{-16}\right)$.
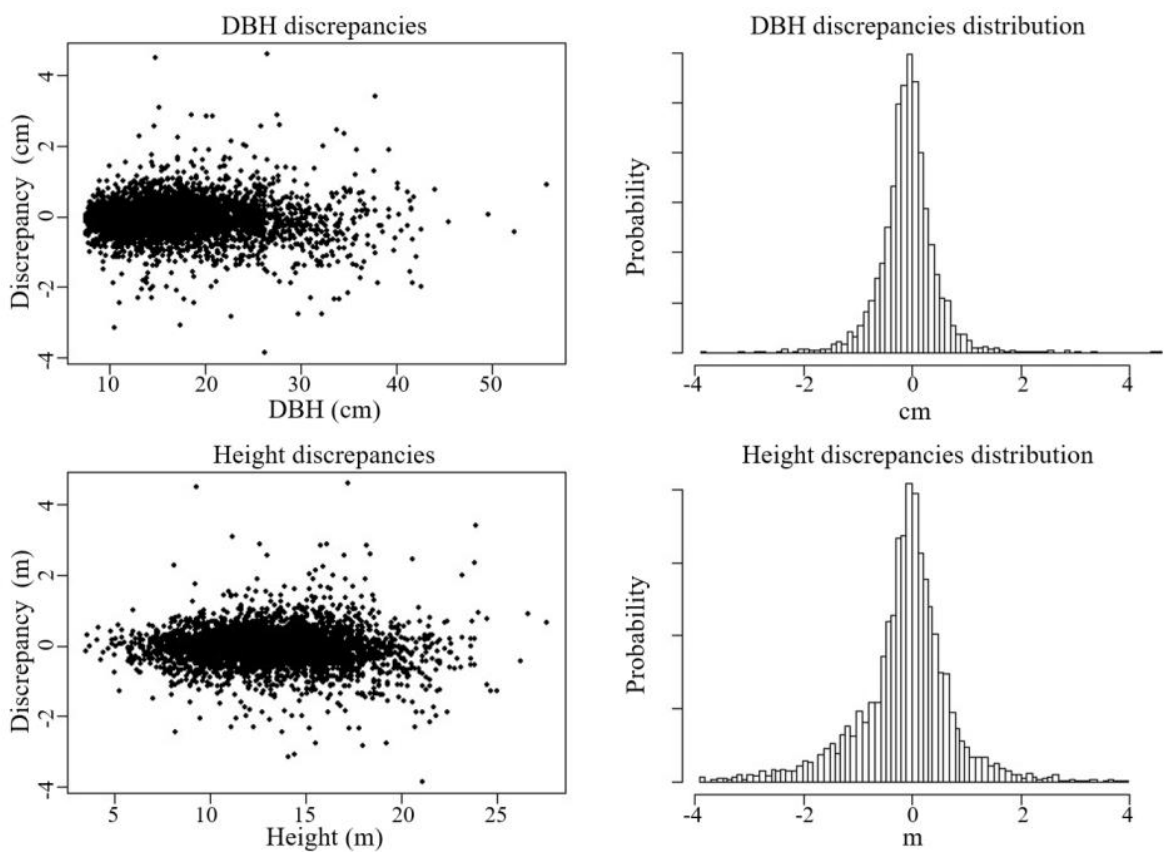

Figure III-4. Discrepancies dispersion across the size of the tree and distribution of the discrepancies for the variables $D B H$ and $T H$ in El Salto, Durango.

To analyze the heteroscedasticity, discrepancies were converted into an absolute value and was fitted a regression line over the values to evaluate the relationship of discrepancy relating to the size of the tree (Figure III-5). The trend line in this figure shows the increase of the discrepancy with the increase of the tree size ( $\mathrm{p}$-value $\mathrm{DBH}_{\mathrm{DB}}<2.2 \mathrm{e}^{-16}, \mathrm{p}$-value $\mathrm{THH}_{\mathrm{TH}}<2.2 \mathrm{e}^{-16}$ ). However, the equations shown here were not further used in the error propagation because the discrepancy is not an uncertainty 
according to the IPCC (2006). They have been provided to illustrate the basic properties of repeated $\mathrm{DBH}$ and $\mathrm{TH}$ measurements.
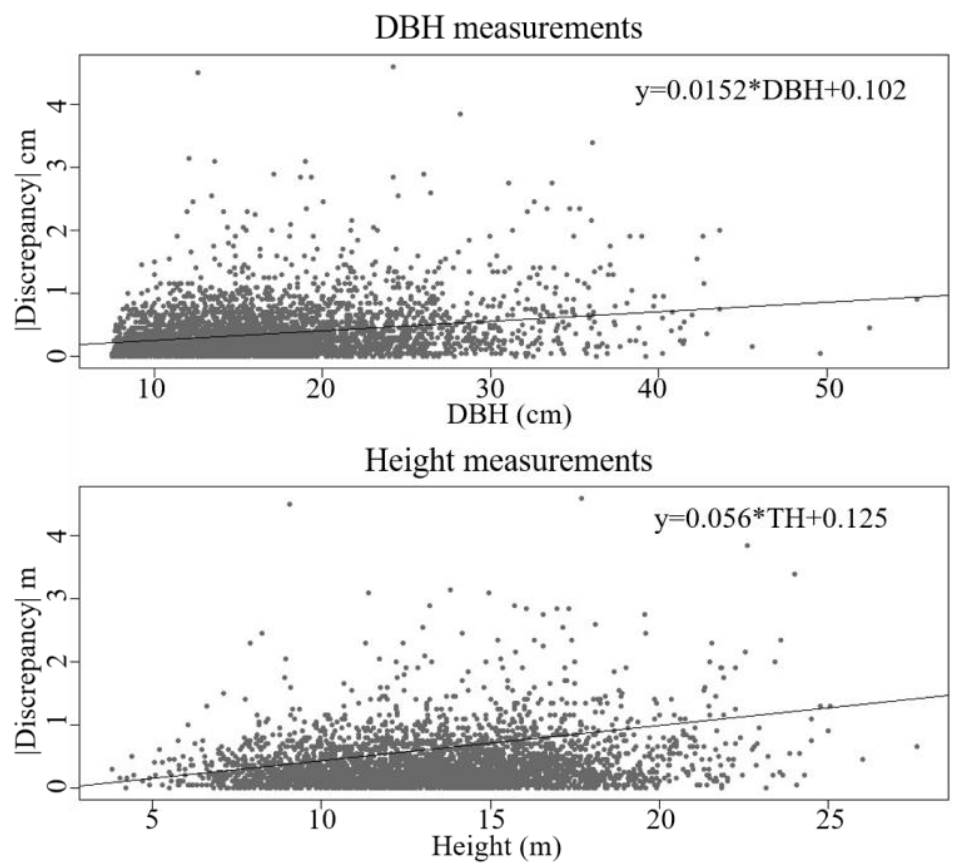

Figure III-5. Linear regression relating discrepancies in measurement with the size of variable measured $(D B H$ and $T H)$.

\section{III.1.5.1.1.1 Methods 1 and 2: Probability Density Function (PDF)}

Discrepancy data was used to construct the uncertainties' distribution. On the left side of Figure III6 , are the plots of the discrepancies, in absolute value. To each discrepancy was added the negative value (mirrored), resulting in the distribution on the right side in Firure III-6. To perform this procedure, we assumed symmetry in the measurement uncertainty. In Method 1, we adjusted a PDF to each data set $(\mathrm{DBH}$ and $\mathrm{TH})$ thus obtaining the parameters to estimate the measurement uncertainty per tree.

In Method 2, to include the uncertainty variability across the tree size, the PDF was adjusted by class (Chave et al., 2004), assuming that the uncertainties behave similarly inside each class of the measured variable. Classes every $5 \mathrm{~cm}$ and $2.5 \mathrm{~m}$ were defined for $\mathrm{DBH}$ and $\mathrm{TH}$ measurements. Every class had more than 100 data to calculate the PDF.

Measurement uncertainties were adjusted to a PDF (Hughes \& Hase, 2010; IPCC, 2003) with the fitDist tool in the "Propagate" package for R (R Core Team, 2018; Spiess, 2015). The adjustment procedure was based on the calculation of the weighted residual sum of squares (RSS) of the observations and is used as an adjustment criterion. Goodness-of-fit (GOF) obtained was the Bayesian information criterion (BIC), which has an increasing number of parameters in the adjustment of the distribution and compensates for the overfitting (Spiess, 2015). RSS and MSE were also part of the $G O F$ report of this package. 


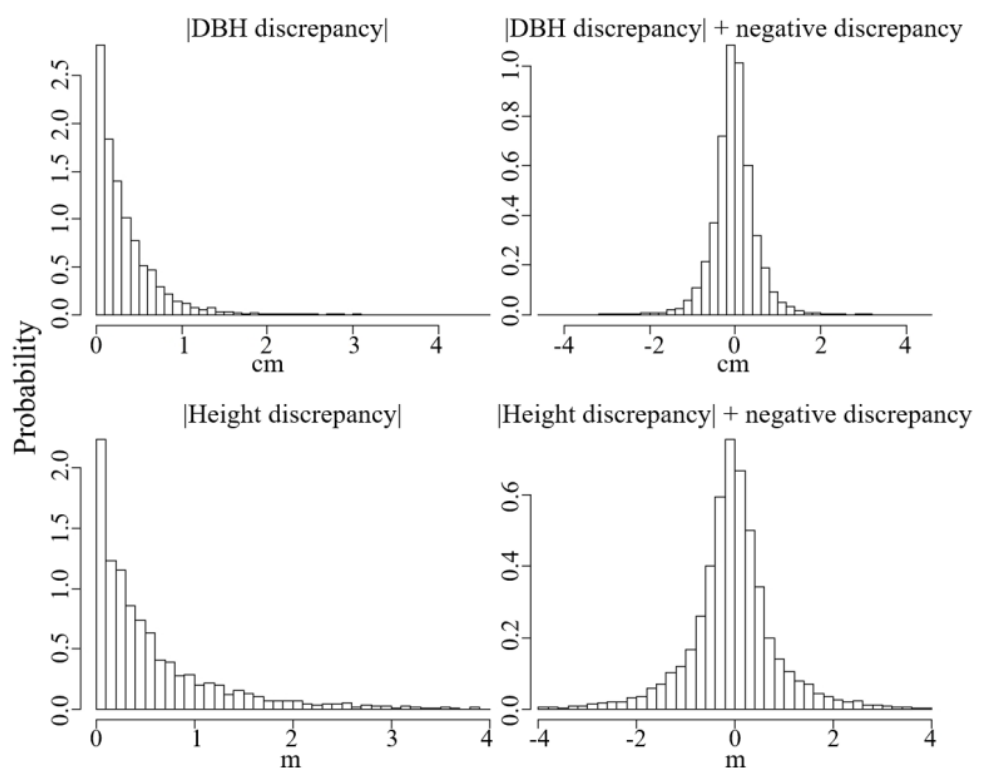

Figure III-6. Probability Density Function of uncertainties for $D B H$ and $T H$ measurements in the study area.

\section{III.1.5.1.1.2 Method 3: Root mean square deviation}

As already mentioned, the discrepancy is not a parameter of uncertainty. However, the discrepancy collected information on the difference between $M 1$ and $M 2$. The root mean square deviation $(R M S D)$ measured the difference between the two observed values per tree, averaging the squared deviations, giving a non-negative result. The Equation that describes the $R M S D$ is the following:

$R M S D=\sqrt{\frac{\sum_{i=1}^{n}\left(x_{M 1}-x_{M 2}\right)^{2}}{n-1}}$

Equation 01

Where: $x_{M 1}-x_{M 2}=$ discrepancy, $n=$ number of trees measured

The $R M S D$ estimated was used as the standard deviation of the uncertainty measurement, assuming a normal distribution and a constant variation (homoscedasticity) not depending on the size of the tree. In Section III.1.5.1.1, it was stated that discrepancies were not normal distributed, however, the normal distribution assumption was necessary in this case to apply the GUM Method of error propagation, using the standard deviation as the parameter of uncertainty applying the Law of Error Propagation.

\section{III.1.5.1.2 Tree-level (prediction error)}

The allometric models were shown in Table III-1. This table included two of the criteria used to select the allometric model to estimate AGB in this study: coefficient of determination $\left(R^{2}\right)$ and $R M S E$. The RMSE was used in the error propagation assuming a normal distribution characterizing the model uncertainty $(u A M)$.

\section{III.1.5.1.3 Sampling error}

The previous sections have described the calculations to obtain the two sources of non-sampling uncertainties used in this empirical study: measurement error and prediction error. In this section, 
we address the equations associated with sampling error, generally, the only source of error reported in forest inventories. Together, sampling error and non-sampling error were the total error in this empirical study.

Simple random sampling (SRS) estimators were used to estimate the parameters of AGB at the stand-level. The estimator for the mean $\hat{\mu}$ estimated the AGB for the study area. The Equation of this estimator is:

mean $(\hat{\mu})=\bar{y}=\frac{\sum_{i=1}^{n} y_{i}}{n}$ Equation 02

Where: $y_{i}=\mathrm{AGB}$ estimated in plot $i ; n=$ number of plots

The variance estimator $\left(\hat{\sigma}^{2}\right)$ describes the dispersion of the per plot AGB values. It is an unbiased estimator of the population variance and was calculated with the following Equation:

variance $\left(\hat{\sigma}^{2}\right)=\frac{\sum_{i=1}^{n}\left(y_{i}-\bar{y}\right)^{2}}{n-1}$

Equation 03

Where: $y_{i}=A G B$ estimated in plot $i ; \bar{y}=$ mean AGB estimate $n=$ number of plots

The standard error of the mean $(S E)$ is the standard deviation $\left(\hat{\sigma}=\sqrt{\hat{\sigma}^{2}}\right)$ of all the possible sample means and is reported in the forest inventories as the sampling error. The $S E$ is used to define the confidence interval in which the parametric mean of the AGB estimation is located with a certain probability of occurrence, commonly $95 \%$.

$S E\left(\hat{\sigma}_{\bar{y}}\right)=\sqrt{\frac{\hat{\sigma}^{2}}{n}}=\frac{\hat{\sigma}}{\sqrt{n}}$ Equation 04

Where: $\hat{\sigma}=$ variance of $\mathrm{AGB}$ estimation; $n=$ number of plots

\section{III.1.5.2 GUM Method of error propagation}

The GUM Method was used to propagate the error in AGB estimation, through the application of the rules for uncertainty assessment of the Joint Committee for Guides in Metrology (JCGM, 2010). Detailed steps of the GUM Method are described below, following the order indicated in Section I.4.2.

\section{i.Specify the measurand to calculate AGB}

The AGB was estimated by an indirect method using allometric models $(A M)$. The predictor variables $(D B H$ and $T H)$ were used in the $A M$ to estimate the AGB per tree. Applying the simple random sampling (SRS) estimators to the AGB values per tree resulted in the AGB in the study area.

\section{ii.Identify the sources of uncertainty}

The sources of uncertainty in AGB estimation were identified in Section III.1.5.1. These sources were grouped as non-sampling error and sampling error. The two sources of non-sampling error were the measurement error and the AGB prediction error.

iii.Quantify uncertainty components or standard uncertainties

Three methods were used to quantify measurement errors, described in Section III.1.5.1.1. With these methods, the measurement uncertainties of $D B H$ and $T H$ per tree were estimated. The AGB 
prediction uncertainty was the $R M S E$ of the allometric models in Table III-1. The $S E$, estimated with Equation 04, was the uncertainty parameter from sampling.

iv. Calculate the value of the AGB

In this step, the step i. was applied to get the best estimate of AGB. This estimate was made from the AGB at tree-level to the AGB at the stand-level, using the sampling error $(S E)$. This estimate of AGB was the baseline excluding non-sampling errors.

v. Calculate the combined uncertainty

The AGB estimate had levels of error propagation or levels of a combination of uncertainties. The first combined uncertainty was the measurement errors in AGB estimation at tree-level (uTree), combining the uncertainty in $D B H(u D B H)$ with the uncertainty in $T H(u T H)$. To combine these uncertainties, the partial contribution of each variable $(D B H$ and $T H)$ in the AGB estimate was calculated (Taylor, 1997). This contribution is the sensitivity coefficient $\left(S C_{D B H}, S C_{T H}\right)$ and was calculated by a partial derivation of the allometric model regarding $\mathrm{DBH}$ and $\mathrm{TH}$ (Equation 05).

$S C_{D B H}=\frac{\partial A G B}{\partial D B H}, S C_{T H}=\frac{\partial A G B}{\partial T H}$

Equation 05

Where: $\frac{\partial A G B}{\partial D B H}=$ partial derivative with respect to $D B H$ of the model used to estimate $A G B ; \quad \frac{\partial A G B}{\partial T H}=$ partial derivative with respect to $T H$ of the model used to estimate $A G B$

The Law of Error Propagation (LEP) is the process that propagates random uncertainties and was used to combine the measurement errors in uMes. This LEP was applied when more than one source of uncertainty was included in the AGB's estimation model. The formula applied considered whether the variables involved were independent (Equation 6a) or dependent (Equation 6b).

$$
\begin{array}{ll}
u \text { Mes }=\sqrt{\left(\left(\frac{\partial A G B}{\partial D B H} u D B H\right)^{2}\right)+\left(\left(\frac{\partial A G B}{\partial T H} u T H\right)^{2}\right)} & \text { Equation 06a } \\
\text { uMes }=\sqrt{\left(\left(\frac{\partial A G B}{\partial D B H} u D B H\right)^{2}\right)+\left(\left(\frac{\partial A G B}{\partial T H} u T H\right)^{2}\right)+\left(\frac{\partial A G B}{\partial D B H} * \frac{\partial A G B}{\partial T H} * u D B H^{*} u T H^{*} \rho_{(D B H, T H)}\right)} & \text { Equation06b }
\end{array}
$$

Where: $u D B H=$ uncertainty of $D B H$ measurements; $u T H=$ uncertainty of $T H$ measurements; $\rho_{(D B H, T H)}=$ correlation coefficient of DBH and TH measurements

The second combined uncertainty was the non-sampling errors in AGB estimation at tree-level (uTree). The uMes was combined with the AGB prediction uncertainty (RMSE in Table III.1). These two uncertainties, independent and random, were combined applying the LEP with Equation 07 .

$$
\text { uTree }=\sqrt{(u M e s)^{2}+(R M S E)^{2}}
$$

\section{Equation 07}

Where: $u M e s=$ measurement uncertainty by tree; $R M S E=$ root mean square error of the allometric model to estimate $A G B$

The third combined uncertainty was the non-sampling errors in AGB estimation at plot-level (uNSplot), by adding uTree in quadrature, as stated in the LEP (Equation 08).

$u$ NSplot $=\sqrt{\left(\text { uTree }_{1}\right)^{2}+\left(\text { uTree }_{2}\right)^{2}+\ldots+\left(\text { uTree }_{n}\right)^{2}}$

Equation 08

Where: uTree $=$ non-sampling uncertainty by tree; $n=$ number of trees 
The fourth combined uncertainty was the non-sampling errors in AGB estimation at the stand-level $(u N S)$. The $u N S p l o t$ were combined to estimate the uNS. Applying the uNSplot in Equation 04, in this combination, was included the sampling design in the estimation of $u N S$.

$u N S=\sqrt{\frac{u \text { Plots }^{2}}{n-1}}=\frac{u \text { Plots }}{\sqrt{n-1}}$

Equation 09

Where: $u$ Plots is $\sqrt{\frac{\sum(u N S p l o t i)^{2}}{n-1}} ;$ uNSplot $=$ uncertainty estimates in the $i$ plot; $n=$ number of plots

Note: To get the contribution to the $u N S$ by a source of uncertainty, only the parameter ( $\hat{\sigma}$ or $R M S E$ ) of the source of interest ( $D B H, T H$ or $A M$ ) was included in the propagation and the other sources were stated without uncertainty. When the contributions by a source of uncertainty were obtained separately, it was verified by the LEP, that the $u N S$ was the product of the independent uncertainties from each source.

$u N S=\sqrt{\left(u_{1}\right)^{2}+\left(u_{2}\right)^{2}+\ldots+\left(u_{i}\right)^{2}}$

Equation 10

Where: $u=$ source of uncertainty; $i=$ number of sources of uncertainty

The fifth and final combination was the total uncertainty in AGB estimation $(u W)$. In this step, the $u N S l$ and the $S E$ were combined (Equation 11). The $S E$ was estimated in the AGB estimation baseline following the step iv.

$u W=\sqrt{u N S^{2}+S E}$

Equation 11

The result of total uncertainty was reported using the $95 \%$ confidence interval. The confidence interval $(C I)$ was the range where the mean AGB of the population was estimated to lie with a $95 \%$ probability (Equation 12). This $C I$ was constructed with the expanded uncertainty ${ }^{4}$ of the AGB estimated $\left(u W_{E X P}\right)$, and this uncertainty was calculated with the $u W$ multiplied by a T-distribution value (Equation 13). Since normality was not assumed with 10 sampled plots, the probability was calculated with the T-distribution. The effective degrees of freedom for T-distribution were calculated with the Welch-Satterthwaite equation (JCGM, 2010; Pérez-Hernández, 2012) (Equation 14)

Confidence interval $=C I_{W 95 \%}=\left(W-u W_{E X P}\right) \leq W \leq\left(W+u W_{E X P}\right)$

Equation 12

Expanded uncertainty $=u W_{E X P}=u W *\left(\mathrm{t}_{0.95}\left(V_{e f f}\right)\right.$ Equation 13

Effective degrees of freedom $=V_{\text {eff }}=\frac{\left(u W^{4}\right.}{\frac{(u N S)^{4}}{d f_{N S}}+\frac{(S E)^{4}}{d f S E}}$

Equation 14

Where: $W=$ total AGB estimate; $u W_{E X P}=$ expanded uncertainty; $u W=$ total uncertainty in the stand; $V_{\text {eff }}$ $=$ effective degrees of freedom; uNS= uncertainty due non-sampling errors; SE=sampling error; $d f=$ degrees of freedom

Figure III-7 shows the flowchart of the steps implemented to propagate the error in the AGB estimation, using the GUM Method.

\footnotetext{
${ }^{4}$ Term used in JCGM (2010) for error propagation with the GUM Method.
} 
i. Specify the AGB estimation

(Explanation of the measurement and the elements in the model, aggregation, estimators)

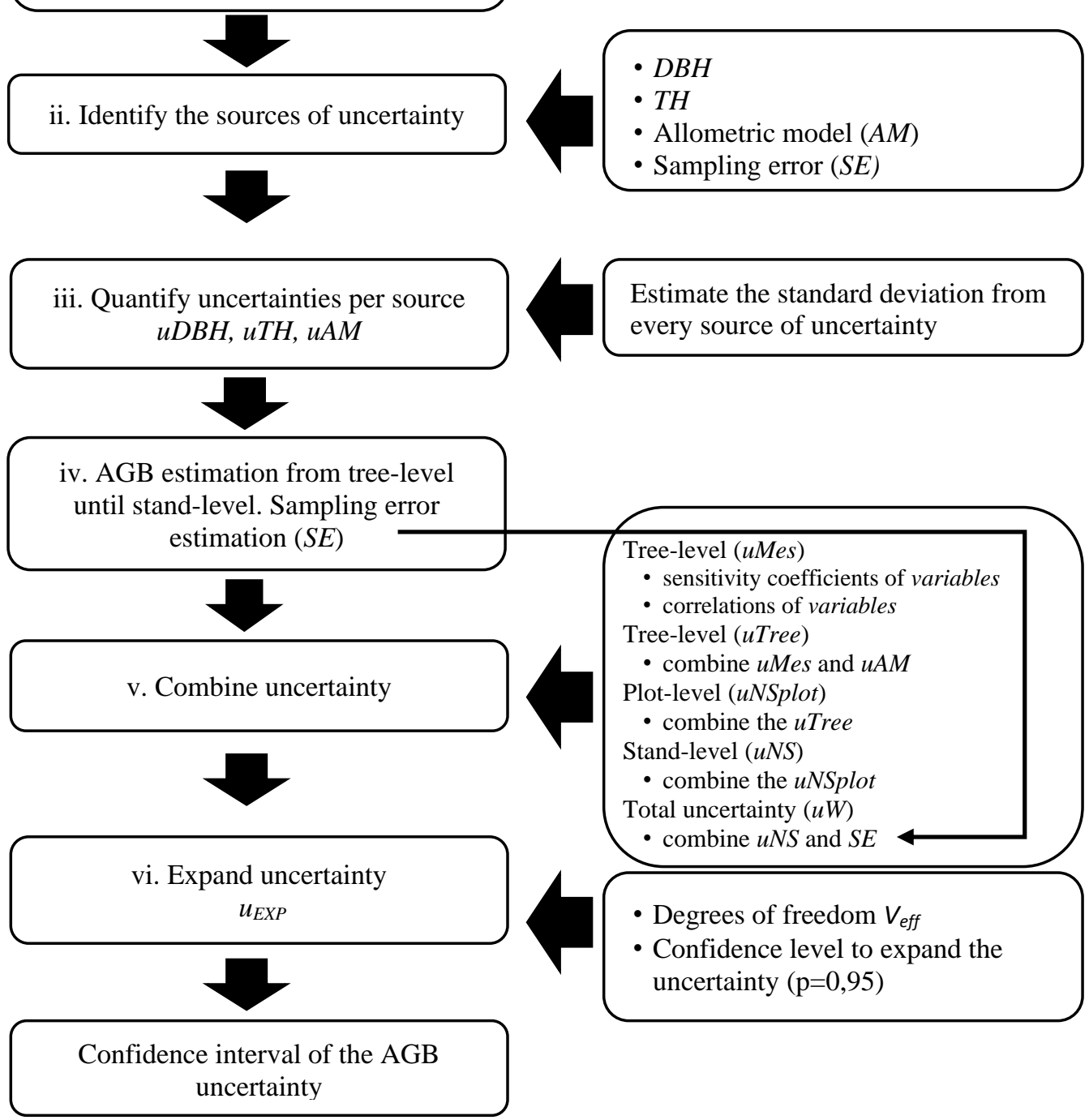

Figure III-7. Flowchart to estimate AGB error propagation adapted from two publications, the Evaluation of measurement by JCGM (2010) and the IPCC Guidelines for National GHG Inventories (2006).

\section{III.1.5.3 Monte-Carlo simulation Method (MCM)}

Determining the criteria for setting the number of iterations for error propagation in AGB estimation (repeated simulations of the estimate) was an important step for the $M C M$. For this study, iterations from 10 to 100000 were evaluated, and the AGB average $\left(\hat{\mu}_{A G B}\right)$, standard error $\left(\hat{\sigma}_{A G B}\right)$, and standard error confidence interval were estimated for each group of reiterations. Following the selection criteria established by the IPCC (2006), the number of iterations from which the value of the estimates reached a variation of less than $1 \%$ of the estimators calculated without uncertainty was selected (Figure III-8). The $M C M$ found stability in the estimation of the 
AGB mean $\left(\hat{\mu}_{A G B}\right)$ estimation and the $S E\left(\hat{\sigma}_{A G B}\right)$ of the AGB estimation from 10000 iterations so that this value was used in all simulations.

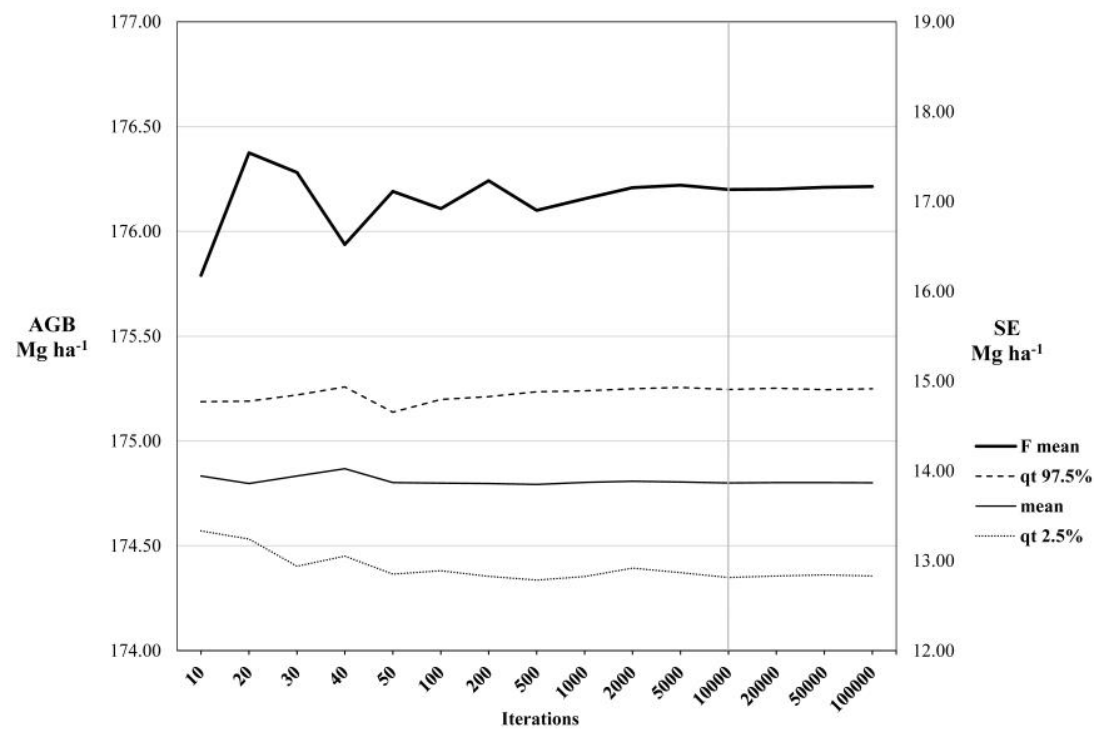

Figure III-8. Iterations made to select the optimal number of iterations for $M C M$ in the uncertainty estimation of AGB with information of El Salto, Durango.

The $M C M$ to estimate the error propagation in AGB estimation was implemented in the Package $\mathrm{R}$ (R Core Team, 2018). The use of the $M C M$ required the parameters of the Probability Density Function (PDF) of measurement uncertainty in $D B H$ and $T H$ (Section III.1.5.1.1.1). The PDF used in $M C M$ was the best evaluated by the Goodness-of-fit: Bayesian information criterion (BIC), residual sum of squares $(R S S)$ and MSE. Correlation of the measured variables $\left(\rho_{(D B H, T H)}=0.78\right)$ was included in $M C M$, because according to Ogilvie (1984) it should be used when it is greater than $|0.8|$, otherwise it is negligible. The AGB prediction error parameter was the RMSE in Table III.1. The flowchart of $M C M$ to propagate the error in AGB estimation is in Figure III.9. This flowchart was made based on the IPCC Guidelines for National GHG Inventories (2006), incorporating the AGB estimate for the uncertainty levels considering under this study. The flowchart represents the steps made by the level of uncertainty and the required information. Step 1 was the PDFs of the non-sampling uncertainty sources used in the AGB estimation (S1L1, S1L2). Step 2 was the random selection of PDF values for measurement uncertainties (S2L1) and allometric model (AM) uncertainty (S2L2). Step 3 was the calculation of the AGB with the $A M$ including the uncertainty values $(S 3 L 1, S 3 L 2)$. In step 4, the iteration process was performed, each AGB estimate was repeated 10,000 times, and the aggregation process from tree-level to plot-level took place. In step 5, using the estimators in Section III.1.5.1.3 were estimated the mean value and the $S E$ of AGB estimate to construct the confidence interval of the error propagation. On the right end of the flowchart is the path used to include all uncertainties in the simulation together. At the bottom are the four outcomes: error propagation with individual uncertainty sources $(u D B H, u T H, u A M)$ and error propagation with all uncertainties. The error propagation using one source of uncertainty at a time provided the contribution of that source to the total uncertainty. The error propagation Equation for the $M C M$ results in non-sampling uncertainties was: 
$u N S \approx \sqrt{(u D B H)^{2}+(u T H)^{2}+(u A M)^{2}}$

Equation 15

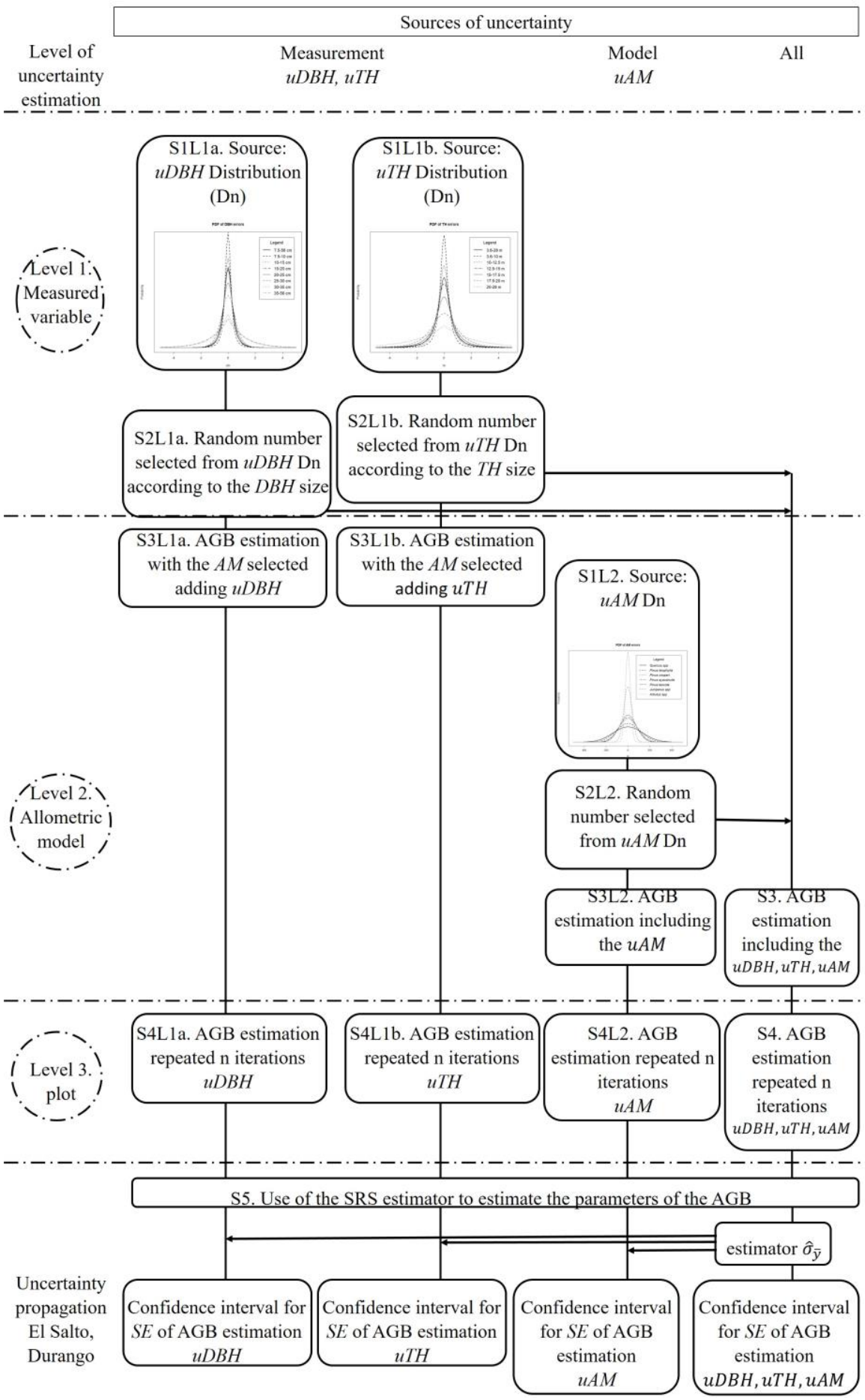

Figure III-9. Flowchart for error propagation of measurement and model uncertainties in El Salto, Durango, Mexico, using the $M C M$. 


\section{III.1.6 Information analysis}

\section{III.1.6.1 General estimations of the study area}

Stand statistics were calculated for AGB and basal area for the study area. Basal area was calculated since it is one of the variables most used in the stand characterization and related to the tree density and AGB (Kershaw Jr. et al., 2017; Lewis et al., 2013; Ni-Meister et al., 2010). Data from the DBH and $T H$, as measurement variables, were included in this analysis.

\section{III.1.6.2 Variables measured and measurement errors}

The relationship between the response variables ( $D B H$ and $T H$ measurements) was analyzed. These variables were used in the allometric model to estimate the AGB. In addition, measurement errors were described using three methods to estimate the measurement uncertainty parameters $(u D B H$ and $u T H)$.

\section{III.1.6.3 Baseline estimation}

AGB estimation, as a reference value, was calculated with the allometric model by species using the mean value $(\hat{\mu})$ of the two measurements for each tree $(D B H$ and $T H)$. The measurement uncertainties and allometric model uncertainties were not required for this estimation.

\section{III.1.6.4 Error propagation}

The three methods used to estimate $D B H$ and $T H$ measurement errors were used to propagate measurement uncertainties in AGB estimation with the GUM Method. After estimating the measurement errors, the prediction errors were aggregated, resulting in the AGB error propagation scenarios. For the identification of the methods used to estimate the measurement error in the propagation of the error, Table III-2 relates the method used with the acronym adopted by the scenario. A similar identification of scenarios was made to the methods used when applying the $M C M$ in error propagation.

\begin{tabular}{lll}
$\begin{array}{c}\text { Error } \\
\text { propagation }\end{array}$ & Methods to estimate measurement error & $\begin{array}{c}\text { Uncertainty } \\
\text { scenario }\end{array}$ \\
\hline \multirow{2}{*}{ GUM Method } & Method 1: Normal distribution & $N D n$ \\
& Method 2: Normal distribution per class & $N D n C$ \\
& Method 3: RMSD & $R M S D$ \\
\hline \multirow{2}{*}{$M C M$} & Method 1: Best PDF & $M C B D$ \\
& Method 2: Best PDF per class & $M C B D C$ \\
\hline
\end{tabular}

Table III-2. Methods and scenarios applied in the error propagation of AGB estimation in El Salto, Durango, Mexico.

The scenarios of error propagations were compared in the errors committed in AGB estimation for the study area. Based on the analysis of error propagation performed, the contribution of uncertainty sources ( $D B H, T H$, and allometric model) to the total error propagation was determined. 
III.2 Empirical study 2: Comparison of aboveground biomass estimates from two types of dasometric measuring equipment for the variables $D B H$ and $T H$, a case study developed in beech forest near Göttingen, Germany

\section{III.2.1 Sampling and plot design}

In this study area, 47 plots were established in the summer of 2015. The plots were laid out in a systematic grid of 75 by $75 \mathrm{~m}$ (Figure III-10). Plots were circular and had an area of $500 \mathrm{~m}^{2}$ (12.61 $m$ radius).
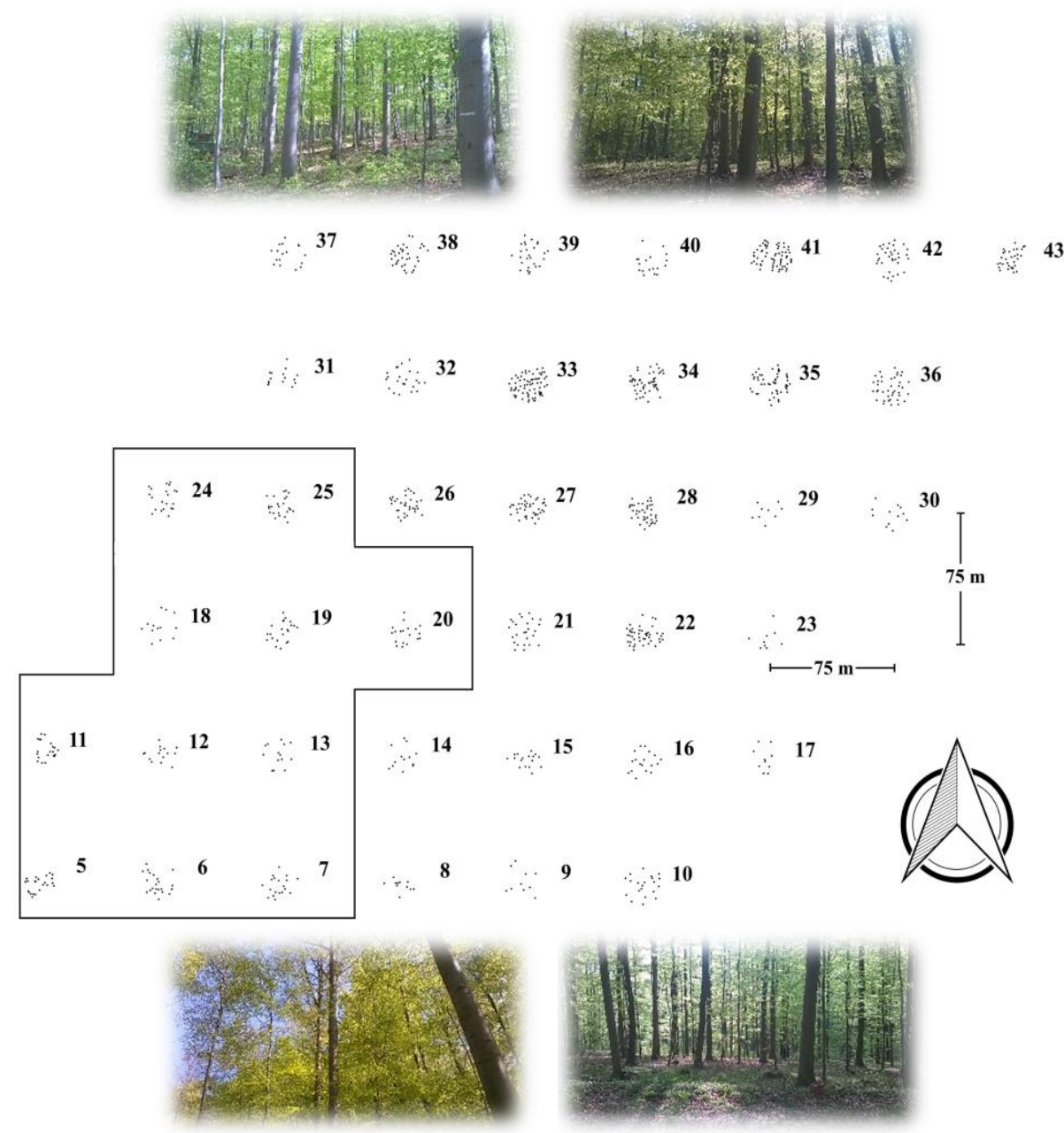

Figure III-10. The pictures give an idea of the study area and the systematic sampling design is given.

\section{III.2.2 Experiment design}

\section{III.2.2.1 Re-measurement fieldwork}

Eleven plots were selected to describe and analyze the measurement uncertainties. Two variables were considered in the fieldwork: $D B H$ and $T H$ as explanatory variables to estimate AGB. The experiment consisted of repeated measurements to estimate the best measurement, or control data, 
per device per variable and to characterize the uncertainty per device per variable. To obtain this control data, two devices were used to measure the $D B H$ and two devices to measure the $T H$.

A protocol of measurement was made for the fieldwork. This protocol established the measurement criteria for the correct usage of the devices including calibration. Applying this protocol, it was assumed that systematic errors were avoided. The measurement protocol was based on the protocol established by FAO (Saket et al., 2004). The devices used for the measurement of the explanatory variables were:

The $D B H$ measurements were made with a Caliper of a maximum of $65 \mathrm{~cm}$ (brand Haglöf) and with a metallic diameter Tape for a maximum diameter of $96 \mathrm{~cm}$. Measurement resolution for both devices was $0.1 \mathrm{~cm}$. A minimum $D B H$ of $7 \mathrm{~cm}$ was defined.

The $T H$ of the trees was measured with two hypsometers, Blume-Leiss (brand Carl Leiss Berlin $\mathrm{GmbH}$ ) and Vertex IV (brand Haglöf). The two devices measured with the trigonometric principle the height of the tree, the measurement unit was in meter $(\mathrm{m})$ and the resolution was 0.5 and $0.1 \mathrm{~m}$, respectively. The accuracy of the Vertex was $1 \%$ and was provided only for horizontal distance (Haglöf Sweden AB, 2007). The resolution was the minimum scale the devices result was read (Schmid \& Lazos Martínez, 2000) and the \% accuracy indicated how different is the device lecture to the true measurement value (Cochran, 1977).

"Blind measurements" were applied (Condit, 1998), as explained in Section III.1.3. Five repetitions were made per device per tree. The repeated measurements made with Tape and Vertex were the responsibility of one observer during the entire experiment, and the repeated measurements with Caliper and Blume-Leiss were made with a second responsible observer. The two responsible for measurements made field data collection in the 11 plots, while one observer conducted the measurements, the other recorded the observations. The devices used for this study were always the same during the data acquisition to avoid bias due to the device. The two observers, before fieldwork, trained to measure the same trees until they reach less than $1 \mathrm{~cm}$ and $1 \mathrm{~m}$ of difference in measurement of $D B H$ and $T H$, respectively.

\section{III.2.2.2 Stand-level fieldwork}

Students of the University of Göttingen registered the field data of the 47 inventory plots. In this data, a single measure per tree and per variable was recorded with Tape and Vertex. This data was used to estimate the AGB and the uncertainty propagation of the measurement errors.

The analysis of the measurement errors between repeated measurements and the fieldwork by students was reported in the master's thesis by Praudel (2015). Praudel compared the measurement of two groups of students (based on their experience in dasometric measurements) and the best estimate in $D B H$ and $T H$ using Tape and Vertex. The results of Praudel (2015) showed that experienced students had measurement uncertainties of $1.18 \mathrm{~cm}$ and $4.01 \mathrm{~m}$ for $D B H$ and $T H$. For non-experienced students, the uncertainties were $1.18 \mathrm{~cm}$ and $4.06 \mathrm{~m}$.

\section{III.2.3 AGB estimation}

The models used in this study, to estimate the AGB, were developed for broad-leaved trees and conifer trees (Fehrmann, 2006). To derive the models, Fehrmann (2006) used data from $n=528$ trees for the general model for broad-leaved trees and data from $n=963$ trees for the general model 
for conifers. The $D B H$ range of the sampled trees was from $0.8 \mathrm{~cm}$ to $77.1 \mathrm{~cm}$ and for tree $T H$ from $1.9 \mathrm{~m}$ to $29.1 \mathrm{~m}$. The corresponding models are in Table III-3, and the total AGB per tree $(W)$ is calculated in $\mathrm{kg}$.

\begin{tabular}{lc}
\hline Vegetation group & \multicolumn{1}{c}{ AGB allometric models } \\
\hline Broad-leaved & $W=0.044 * D B H^{2.048} * T H^{0.759}$ \\
Broad-leaved & $W=0.099 * D B H^{2.501}$ \\
Conifers & $W=0.082 * D B H^{2.170 * T H^{0.349}}$ \\
Conifers & $W=0.089 * D B H^{2.464}$ \\
\hline
\end{tabular}

Table III-3. Allometric AGB models used in the study area of Göttingen, Germany (Source: Fehrmann, 2006).

\section{III.2.4 Sources of uncertainties}

\section{III.2.4.1 Tree-level (measurement error)}

\section{III.2.4.1.1 Individual tree uncertainty.}

The SRS estimators were used to calculate the mean value $(\hat{\mu})$ and the standard deviation $(\hat{\sigma})$ of the five measurements per tree, in the 11 re-measurement plots. The mean $(\hat{\mu})$ was the best estimate value of the measurement and the standard deviation $(\hat{\sigma})$ was the uncertainty parameter of the measurement. Applying these calculations to the measured variables ( $D B H$ and $T H$ ), and in the four devices used, were estimated the measurement uncertainty parameter per variable, per device, and per tree. Although this uncertainty was an estimate, it was named "measured uncertainty" indicating that it comes from the repeated measurement per tree.

\section{III.2.4.1.2 Methods to estimate the "measured uncertainty"}

Repeated measurements were made on 11 out of 47 plots, i.e. the "measured uncertainty" could not be estimated for all trees. Therefore, four methods were used to estimate the measurement uncertainty parameters to include this uncertainty in all the trees at the stand-level. The source of information was the "measured uncertainty" (previous section) and the methods applied are described in the following sections.

\section{III.2.4.1.2.1 Method 1: mean value of "measured uncertainty"}

The mean value or average of the "measured uncertainty" from Section III.2.4.1.1 was calculated, and this mean uncertainty parameter $(\sigma)$ was applied to the measurements made in trees of the 47 plots. Applying this method, we assumed that the measurement errors had the same size $(\sigma)$ on all trees and these errors not depended on the size of the tree. This estimation was made per device.

\section{III.2.4.1.2.2 Methods 2 and 3: Probability Density Function (PDF)}

These methods were explained in Section III.1.5.1.1.1. In this case study, the uncertainty information to calculate the Probability Density Functions (PDF) was the standard deviation ( $\hat{\sigma})$ estimated per tree or "measured uncertainty". In the method 2, a PDF was fitted using all the dataset of measurement errors $(\hat{\sigma})$ and was applied per variable measured $(D B H$ and $T H)$ and per device. In 
method 3, the total number of measurement uncertainties $(\hat{\sigma})$ was divided into three classes. The classes used were 7-20, 20-40, 40-63 for $D B H$ (in centimeters) and 5 - 15, 15 - 25, 25 - 37 for TH (in meters). The criteria used for the selection of classes was taken from Pardé and Bouchon (1988), using classes with more than 50 observations to fit a PDF per class, considering every class as a homogeneous dataset.

\section{III.2.4.1.2.3 Method 4: Regression analysis}

The relationship between the $D B H$ and its measured uncertainty was adjusted to a linear model and the Shapiro-Wilkoxon and Breusch-Pagan Tests were applied to verify the normality and homoscedasticity of the regression residuals. The hypotheses of normality and heteroscedasticity $\left(\mathrm{p}_{\mathrm{SW}}=3.612 \mathrm{e}^{-12}\right.$ and $\left.\mathrm{p}_{\mathrm{BP}}=3.2 \mathrm{e}^{-06}\right)$ were rejected. Classical transformations $\left(\ln \mathrm{x}, \sqrt{\mathrm{x}}_{\mathrm{x}}, 1 / \mathrm{x}\right)$ applied to the data to meet the assumptions of normality were not successful, so that a transformation of the response variable was performed through the Box-Cox method (Box \& Cox, 1964; García-Pérez, 2014). Once the transformation was applied, the residuals had a normal distribution; however, the heteroscedasticity was significant. Due to heteroscedasticity, a weight factor was incorporated into each observation (inversely proportional to the variance) to perform a regression with the Weighted Least Squares estimator (wls). This estimator incorporated the variability of the errors into the model and estimated a homoscedastic error model (Fahrmeir et al., 2013; Hill et al., 2018). For model assessment, cross-validation was made (James et al., 2013). One sample of half the dataset was randomly selected and used to calculate the test error rate of the model. The selection process was repeated 1000 times to construct the $95 \%$ confidence interval of the MSE, verifying that the MSE of the fitted model was in the estimated confidence interval.

\section{III.2.4.2 Tree-level (prediction error)}

The Goodness-of-fit reported for the allometric models used in this study are shown in Table III-4. The RMSE described the contribution of the model uncertainty to the total uncertainty, this was the uncertainty due to the model ( $u A M)$.

\begin{tabular}{lccrc}
\hline \multicolumn{1}{c}{ Vegetation Group } & $\mathbf{n}$ & $\boldsymbol{R}^{\mathbf{2}}$ & $\boldsymbol{R M S E}(\mathbf{k g})$ & Source \\
\hline Broad-leaved $(D B H, T H)$ & 528 & 0.98 & 1.30 & \\
Broad-leaved $(D B H)$ & 528 & 0.98 & 1.35 & Fehrmann, 2006 \\
Conifers $(D B H, T H)$ & 963 & 0.97 & 1.31 & \\
Conifers $(D B H)$ & 963 & 0.97 & 1.32 & \\
n=number of sampled trees, $R^{2}=$ coefficient of determination and $R M S E=$ root mean square error.
\end{tabular}

Table III-4. Goodness-of-fit for the models used in the study area of Göttingen using $D B H$ and $T H$ measurement.

\section{III.2.4.3 Sampling error.}

In this study, was used a systematic sampling in the fieldwork to collect the data. The estimator of the mean $(\hat{\mu})$ was the estimator described for simple random sampling (SRS). On variance and $S E$ no unbiased estimators have been developed for systematic sampling (Mostafa \& Ahmad, 2018). However, the variance estimator $\left(\hat{\sigma}^{2}, \hat{\sigma}_{\bar{y}}\right)$ of the SRS in the data obtained with systematic sampling is generally used, even when the estimators of SRS overestimate the variance due to systematic design 
is more precise (Cochran, 1977). SRS estimators were used for this study and the equations have been addressed in the previous case study (Section III.1.5.1.3).

\section{III.2.5 Monte-Carlo simulation Method (MCM)}

The MCM was used to propagate the error in AGB estimation of the study area. The MCM was conducted for the two measurement datasets, Caliper and Blume-Leiss $(C B)$ and Tape and Vertex (TV) measurements. The flowchart used in the first topic (Figure III-9, Section III.1.5.3), was basically the same used to represent the $M C M$ in the case study of Göttingen. The difference was the use of two datasets of measurement error to estimate the AGB ( $C B$ and $T V$ ). The following Section III.2.6 lists the datasets and methods to estimate measurement errors used in $M C M$.

The MCM approximations, as a result of the applied iterations, had a difference of less than $1 \%$ in the estimators (mean $\left(\hat{\mu}_{A G B}\right)$ and SE $\left.\left(\hat{\sigma}_{A G B}\right)\right)$ to be valid, according to the guidelines of the IPCC (2006). Figure III-11 shows the result of the test carried out with the data of the study area in Göttingen finding 10000 as the number of iterations used in this study. This number of iterations was used to estimate the AGB in the scenarios evaluated in this case study.

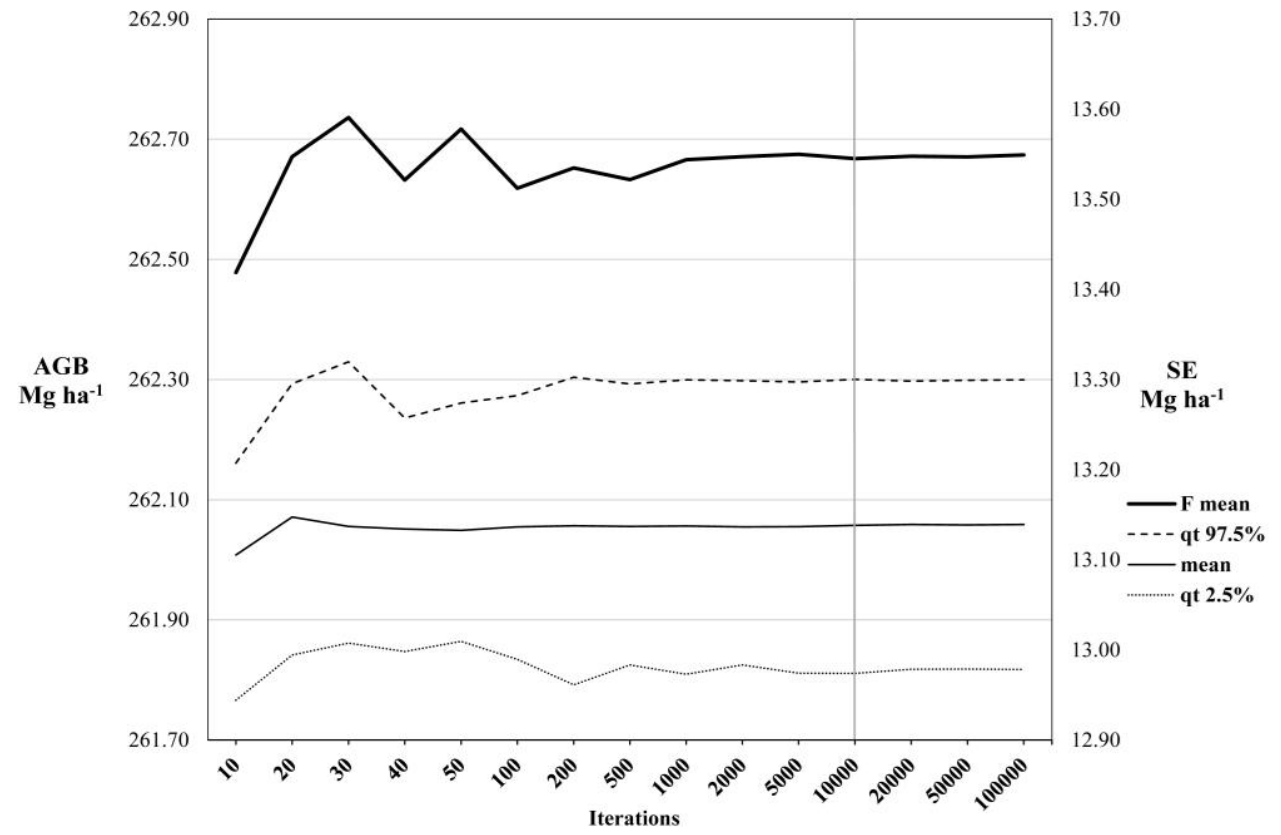

Figure III-11. Essay of iterations to select the optimal number of iterations for $M C M$ in the uncertainty estimation of AGB with information of Göttingen.

\section{III.2.6 Information analysis}

\section{III.2.6.1 General estimators of the study area}

AGB and basal area statistics were calculated for stand area (47 plots) and re-measurement area (11 plots). In the re-measurement plots were calculated the statistics for two datasets, Caliper+BlumeLeiss $(C B)$ and Tap+/Vertex (TV). This analysis included statistics of the measured variables $D B H$ and $T H$. 
The relationship between the variables used to estimate the AGB at the re-measurement plots was compared and analyzed for the two datasets $(C B$ and $T V)$.

The measurement errors of each measuring device were described and the parameters of the PDF describing the measurement errors were estimated. This was done for the whole dataset and for the $D B H$ and $T H$ classes, according to the measurement device.

\section{III.2.6.2 Baseline information}

In this empirical study, four-baseline datasets of AGB without uncertainty were estimated (Table III.5) in the 11 re-measurement plots. The datasets used were a combination of the measurement device(s) and the allometric model to estimate the AGB. The measurement per device used in the estimation were the mean values per tree (best estimate).

\begin{tabular}{lllc}
\hline $\begin{array}{c}\text { Measurement } \\
\text { variable }\end{array}$ & \multicolumn{1}{c}{ Device (s) } & Allometric model & Dataset \\
\hline$D B H, T H$ & Caliper, Blume-Leiss & $\mathrm{AGB}=f(D B H, T H)$ & $C B$ \\
$D B H, T H$ & Tape, Vertex & & $T V$ \\
$D B H$ & Caliper & $\mathrm{AGB}=f(D B H)$ & $C$ \\
$D B H$ & Tape & & $T$ \\
\hline
\end{tabular}

Table III-5. Reference datasets in AGB estimation for re-mesurement plots.

The baseline information used in the stand area, recorded by students (Section III.2.2.2) was used to estimate the AGB applying the allometric models for conifers and hardwoods to the measurement variables (best estimate).

\section{III.2.6.3 Error propagation}

The first analysis was performed on the 11 re-measurement plots using the baseline information (Section III.2.4.1.2). In this analysis, the "measured uncertainty" was included, and the GUM Method of error propagation was applied to estimate the total uncertainty in AGB estimation for the datasets in Table III-5.

The second analysis, also on the 11 re-measurement plots, compared the scenarios of error propagation with GUM Method proposed in this study. The scenarios included two sources of information, the dataset $(C B$ or $T V$ ) and the method used to estimate measurement uncertainty. Table III- 6 contains the acronyms to identify the scenarios to easily identify them in the results section, the measurement error of the students were also included as scenarios. To compare the methods, an Analysis of Variance for repeated measurements were used and paired T-Tests were applied to determine significance in paired comparisons.

The third analysis was conducted with information from the 47 plots. The scenarios that best represented the "measured uncertainty" in the previous analysis were selected. These scenarios, in addition to the scenarios that used the best PDF to describe the measurement error, were used to propagate the error using the $M C M$ and were compared. An Analysis of Variance for repeated measurements and paired T-Tests were used to compare the scenarios and find significance in the paired comparisons. Table III-7 lists the scenarios used in the $M C M$, including the students' measurement error as scenarios. 


\begin{tabular}{cll}
\hline Dataset & \multicolumn{1}{c}{$\begin{array}{c}\text { Measured uncertainty and } \\
\text { Methods to estimate measurement uncertainty }\end{array}$} & $\begin{array}{c}\text { Uncertainty } \\
\text { scenario }\end{array}$ \\
\hline \multirow{4}{*}{$C B$} & Measured uncertainty & $C B$ \\
& Method 1: mean & CBmean \\
& Method 2: Normal distribution & CBNDn \\
& Method 3: Normal distribution per size class & CBNDnC \\
& Method 4: Regression model & CBmod \\
\hline \multirow{6}{*}{$T V$} & Measured uncertainty & TV \\
& Method 1: mean & TVmean \\
& Method 2: Normal distribution & TVNDn \\
& Method 3: Normal distribution per size class & TVNDnC \\
& Method 4: Regression model & TVmod \\
& Students with prior experience & TVExp \\
& Students without prior experience & TVNexp \\
\hline
\end{tabular}

Table III-6. Scenarios of error propagation with GUM Method.

\begin{tabular}{cll}
\hline Dataset & Methods to estimate measurement uncertainty & $\begin{array}{c}\text { Uncertainty } \\
\text { scenario }\end{array}$ \\
\hline \multirow{2}{*}{$C B$} & Method 2: Best PDF & $C B B D$ \\
& Method 3: Best PDF per size class & $C B B D C$ \\
\hline \multirow{3}{*}{$T V$} & Method 2: Best PDF & $T V B D$ \\
& Method 3: Best PDF per size class & $T V B D C$ \\
& Students with prior experience & TVExp \\
& Students without prior experience & $T V N \exp$ \\
\hline
\end{tabular}

Table III-7. Scenarios of error propagation with $M C M$.

Error propagation results by each source of uncertainty were reported, estimating the contributions of uncertainty in measuring $D B H(u D B H)$, uncertainty in measuring $T H(u T H)$, AGB prediction uncertainties $(u A M)$ and sampling uncertainties $(S E)$. Table III-8 shows the identification of sources of uncertainty for the $C B B D$ scenario, also used in the other scenarios.

\begin{tabular}{clll}
\hline Dataset & $\begin{array}{c}\text { Methods to estimate } \\
\text { measurement uncertainty }\end{array}$ & Source of uncertainty & $\begin{array}{c}\text { Uncertainty } \\
\text { scenario }\end{array}$ \\
\hline \multirow{4}{*}{$C B$} & & $a l l$ & $C B B D$ \\
& Method 2: Best PDF & $u D B H$ & $C B B D a$ \\
& & $u T H$ & $C B B D b$ \\
& & $u A M$ & $C B B D c$ \\
& & $S E$ & $C B B D d$ \\
\hline
\end{tabular}

Table III-8. Sources of error contributing to error propagation scenario.

III.3 Empirical study 3. Propagation of errors in the AGB estimation for the state of Durango, Mexico, with information of the National Forest Inventory

\section{III.3.1 Overview}

The information registered in MNFI is assumed free of measurement uncertainties and only the sampling error is reported in the variables estimated. The information analyzed and the results obtained in El Salto, Durango (first empirical study), were important in describing the measurement 
uncertainty in $\mathrm{DBH}$ and $\mathrm{TH}$, because there have been no studies in Durango that provide this information. In this empirical study, it was assumed that the measurement uncertainties in MNFI were like the measurement uncertainties assessment described in the El Salto, Durango, to propagate the uncertainties in AGB estimation, using the Monte Carlo simulation Method.

\section{III.3.2 Fieldwork dataset to estimate AGB}

Two datasets were used for this study; the first measurement of the MNFI in the state of Durango for the period 2004-2009 and the first re-measurement from 2009-2014. The registers were made following the "Manual and procedures for field sampling" made by CONAFOR to be applied at the fieldwork stage (CONAFOR, 2009b).

\section{III.3.3 Sampling Design}

The sampling design used in the MNFI was a systematic stratified sampling using a cluster of four subplots in a fixed pattern. A grid composed of panels of $5 \times 5 \mathrm{~km}$ was superimposed on the territory of Mexico (Figure III-12). Each point in this grid represented the location of one cluster. According to the vegetation type (INEGI, 2017), and forest density the grid was applied with wider distances (10 X $10 \mathrm{~km}$ and $20 \mathrm{X} 20 \mathrm{~km})$. CONAFOR, in the field manual, named the cluster as a primary sampling unit ( $P S U$ ) and the subplot as a secondary sampling unit ( $S S U$ ) The $S S U$ was where the variables and characteristics of the site were measured/estimated/described. The description of the size and shape of the PSU and $S S U$ is detailed below (CONAFOR, 2012c)

\section{III.3.4 Plot design}

In 1998, the North American Science Symposium was held with the aim of standardizing methods of data collection in future monitoring programs and inventories of forest in North America, to make them comparable and with assured quality. In this symposium, the plot design of the United States Forest Inventory (Bechtold \& Zarnoch, 1999) was described, as well as proposals for plot designs to optimize forest sampling (Schreuder \& Geissler, 1999). With this precedent, and for the new MNFI, Velasco et al. (2002) evaluated seven plot designs for the variables: number of trees, basal area, and volume. According to the analysis of Velasco et al. (2002), the optimal plot design was applied in the MNFI with a relative error of less than 7\%. This design, consisted of a PSU of one hectare with a radius of $56.42 \mathrm{~m}$, inside this $P S U$ four $S S U$ of $400 \mathrm{~m}^{2}$ each with a fixed radius of $11.28 \mathrm{~m}$ were sampled. Figure III-13 is shown the aspect of the PSU, and the inverted "Y" design in where the four $S S U$ were arranged in fieldwork (CONAFOR, 2012c).

In the $S S U$, were measured trees with $D B H$ equal to or greater than $7.5 \mathrm{~cm}$. Inside the $S S U$, in a nested subplot of $12.56 \mathrm{~m}^{2}$ ( 2 m radius), were measured trees with $D B H$ less than $7.5 \mathrm{~cm}$ with a height greater than or equal to $25 \mathrm{~cm}$. In the center of the $S S U$ was located one-square-meter site micro-plot to sample herbs, ferns, mosses, and lichens. In total, 112 variables were measured/estimated/observed in the MNFI, 54 variables for quantitative purposes and 58 for qualitative (CONAFOR, 2009a, 2012c). 


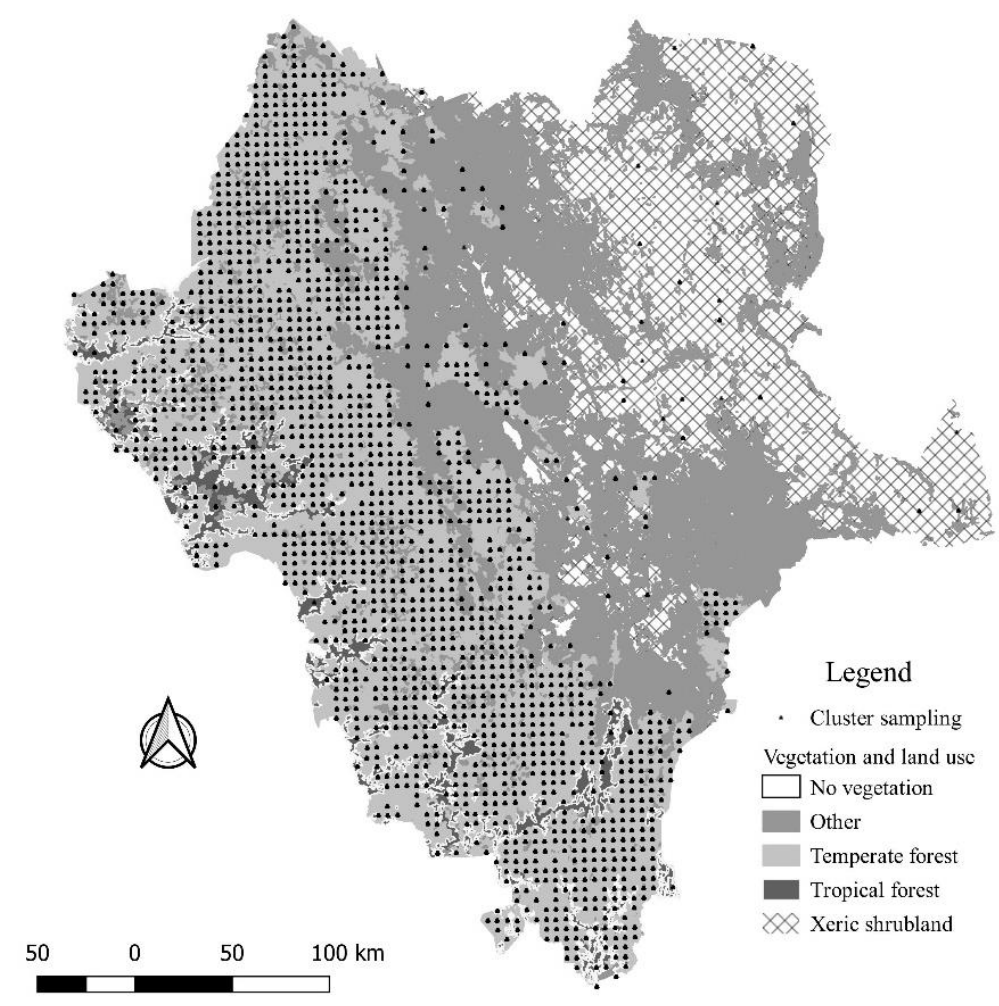

Figure III-12. Durango coverage grid for the tree main vegetation types.

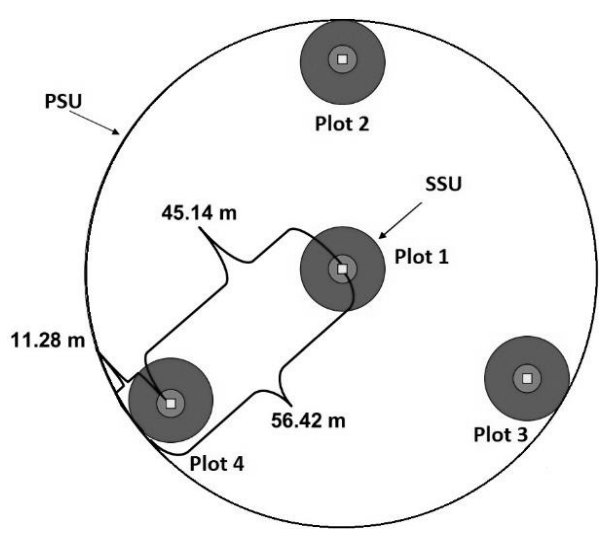

Figure III-13. Form and distribution of PSU and SSU in the MNFI in Mexico. Source (CONAFOR, 2012c).

\section{III.3.5 Estimators design}

The estimators used in the MNFI were designed by Velasco et al. (2003) using in the research the main variables of interest (basal area, tree density, volume, among others) of the MNFI (SEMARNAT, 2004) and adding the hierarchical level of estimation for vegetation types: ecosystem, stratum, and substratum (INEGI, 2014). The estimator was a ratio estimator using the area of the $S S U$ as an auxiliary variable to obtain the results of the parameters measured in hectares. The estimators used the following notation (CONAFOR, 2012b):

Ratio estimator 
$\widehat{R}_{h}=\frac{\sum_{i=1}^{n_{h}} Y_{h_{i}}}{\sum_{i=1}^{n_{h}} A_{h_{i}}}$

Equation 16

Where: $\widehat{R}_{h}=$ Estimated ratio to obtain information at stratum level or vegetation formation

$n_{h}=$ Number of $P S U$ evaluated in stratum $h$

$Y_{h_{i}}=$ Variable of interest (AGB or basal area) $=\sum_{j=1}^{m_{j}} Y_{h_{i j}}=\sum_{j=1}^{m_{j}} \sum_{k=1}^{t_{h i j}} Y_{h_{i j k}}$

$Y_{h_{i j}}=$ Value of the variable of interest in $S S U j$ (secondary sampling unit-SSU-) of PSU $i$ of stratum $h$

$Y_{h_{i j k}}=$ Value of the variable of interest of tree $k$ in $S S U j$ of $P S U i$ of stratum $h$

$t_{h i j}=$ Number of trees evaluated in $S S U j$ of $P S U i$ of stratum $h$

$m_{j}=$ Number of the $S S U$ evaluated in the $P S U ; j=1,2,3$ or 4

$A_{h_{i}}=$ plot area (ha) sampled from $P S U i$ of stratum $h$ (auxiliary variable) $=\sum_{j=1}^{m_{j}} A_{h i j}$

$A_{h i j}=$ Area (0.4 ha) of $S S U j$ of $P S U i$ of stratum $h$

The variance estimator measured how spread out was the variability from the ratio estimation in the variable of interest.

$\hat{\sigma}^{2}(\widehat{R})=\frac{\hat{\sigma}_{Y_{h_{i}}}^{2}+\widehat{R}^{2} \hat{\sigma}_{A_{h_{i}}}^{2}+2 \widehat{R} \hat{\sigma}_{Y_{h_{i}}{ }^{A} h_{i}}}{n \bar{A}_{h_{i}}^{2}}$

Equation 17

Where: $\hat{\sigma}_{Y_{h_{i}}}^{2}=$ Variance of the variable of interest (AGB or basal area)

$\hat{\sigma}_{A_{h_{i}}}^{2}=$ Variance of the auxiliary variable (plot area)

$\hat{\sigma}_{Y_{h_{i}} A_{h_{i}}}=$ Covariance of the variable of interest and the auxiliary variable (sampled area)

$\bar{A}_{h_{i}}=$ Mean value of forest area (ha) sampled

The standard error was the square root of Equation 17.

$S E(\widehat{R})=\sqrt{\hat{\sigma}^{2}(\widehat{R})}$

Equation 18

The relative standard error expresses the standard error in percent of the estimated mean:

$S E \%=R S E(\widehat{R})=\frac{\sqrt{\widehat{\sigma}^{2}(\widehat{R})}}{\widehat{R}} * 100$

Equation 19

\section{III.3.6 National Forest Inventory data validation}

A review process of the raw MNFI database was applied, as a validation system, to detect registry errors and select the information to be analyzed (Morales M., 2005). Since the original field sheets were not accessible, it was not possible to determine typing and writing errors. However, a debugging of the database and a selection of the records were made, following the scheme presented below (Figure III-14). The debugging and selection were made under the assumption that the errors occurred during data typing in the database program. 


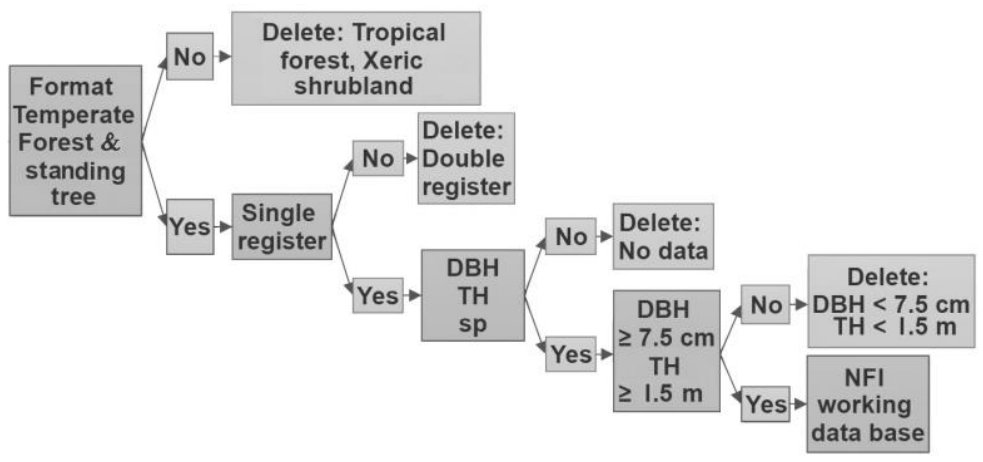

Figure III-14. Flow diagram for database debugging implemented to select the information to analyze from the database of MNFI in Durango.

The database debug was designed to select the forest type, standing trees for this case study, and delete the registers with mistakes. The debug had as a first criterion the selection of temperate forest clusters, the predominant vegetation in the study area. As the second criterion the standing alive trees were selected, due to the allometric models used to estimate the AGB were fitted with these criteria. The next step consisted of the elimination of duplicated registers and registers without information of $D B H$, height or without species identification. During this process were eliminated records with $D B H$ less than $7.5 \mathrm{~cm}$ due they did not comply with the regulations of the data collection protocol and were considered records mistake.

As part of the debugging of the database, graphical analyses were performed for each tree species using the relationship $D B H$ and height to compare with the information of the technical datasheets made by CONAFOR and in this way rule out errors of registration assignment ( $D B H, T H$, species). The graphic analysis was complemented by applying the non-parametric method used by $\mathrm{Bi}(2000)$, to locate outliers in the $D B H \sim T H$ relationship.

\section{III.3.7 AGB estimation}

The AGB estimate for the MNFI data in Durango was made using allometric models $(A M)$ for the 346 species registered. Mexico's dataset of models to estimate AGB does not have complete coverage for all tree species of Durango (Rojas-García et al., 2015). Therefore, using the model selection method proposed by CONAFOR (2014), the species were grouped by vegetation type or genus to estimate the AGB. The selection criteria and model assignment was explained in Section III.1.4.

The 36 models used to estimate the AGB are shown in Table VIII.1 of Appendix I. From the models used, six were produced in studies conducted in Durango (Arbutus sp, Juniperus spp, Pinus spp, Pseudotsuga spp, Prosopis spp, Quercus spp) and used to estimate the AGB for $96 \%$ of the trees sampled in the MNFI. The genera with the highest share were Quercus spp with $44 \%$ of the registered trees and Pinus spp (37 species) with 40\%. For Cedrela spp, Yucca spp, tropical dry forest and desert communities, $A M$ from neighboring states of Durango were used. Together, these four vegetation types represented $1.9 \%$ of the sampled trees; however, these included 144 species, mainly shrubs. The remaining $26 A M$ used were for genera and species with the lowest number of observations at the MNFI in Durango (2.6\%). For these genera and species, no studies are reported 
that have built $A M$ for AGB estimation, so adjusted $A M$ were used in other states of Mexico and even in other countries.

\section{III.3.8 Source of uncertainties}

\section{III.3.8.1 Tree-level (measurement error)}

The information assessed in El Salto, Durango (first empirical study), was used to estimate the measurement uncertainties of the trees in the MNFI in Durango State. The distribution of the trees measured in El Salto, MNFI 2004-2009 and MNFI 2009-2014 is shown in Figure III-15. In this figure, due to the scale and density of the points, the trees less than $20 \mathrm{~m}$ of $T H$ and less than $30 \mathrm{~cm}$ of $\mathrm{DBH}$ are overlayed.

The Figure III-15 shows two differences between the dataset in El Salto compared to the datasets of the MNFI in Durango State: the maximum limit of the measured THs and $D B H s$, and the clearly limited subset for the trees in El Salto. These differences were because sampling in El Salto was made in relatively homogeneous pine plantations without forest management, while in MNFI, the objective was to determine the variability of the species and the potential productivity of all the forests in Durango state and generally in Mexico (CONAFOR, 2017a).

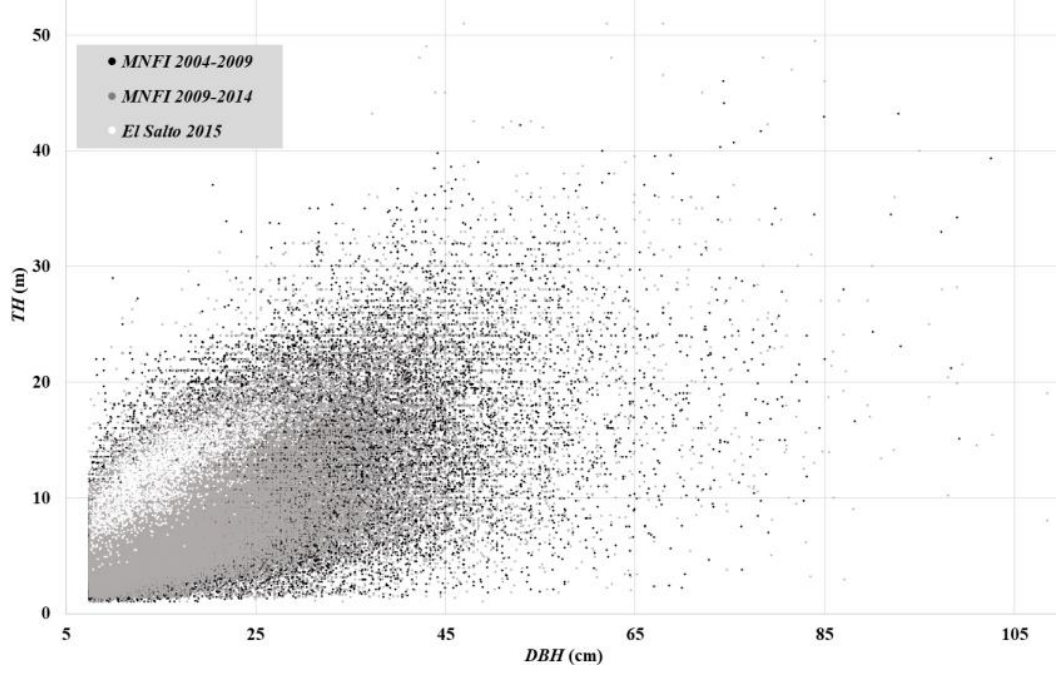

Figure III-15. Tree distribution in the relationship $D B H(\mathrm{~cm}) \& T H(\mathrm{~m})$ for El Salto 2015 $(\mathrm{n}=4262)$, MNFI 2004-2009 ( $\mathrm{n}=109762)$ and MNFI 2009-2014 $(\mathrm{n}=109983)$.

\section{III.3.8.2 Tree-level (prediction error)}

The allometric models used to estimate the AGB were selected according to previously established criteria. One of these criteria was the MSE, which expressed the variability of the data estimated by the model. Table VIII.1, with the MSE values by allometric model, is in Appendix I of this document. This table shows that 16, of 36 models used were reported with the MSE value. These 16 models covered about $98 \%$ of the MNFI sample trees. 


\section{III.3.8.3 Plot design error}

The plot design implemented in the MNFI had two major characteristics: 1) relative error of less than $7 \%$ as a previous-established criterion in the study area (Velasco et al., 2002) and 2) this design reduced implementation costs according to previous studies by Scott (1993). The design used in the MNFI was the optimal but also the one with the highest relative error and according to Velasco et al. (2002), there were designs with $2 \%$ less relative error. The $2 \%$ variation was used to propagate the uncertainty due to the plot design assuming that this uncertainty was normally distributed.

\section{III.3.9 Error propagation with Monte-Carlo simulation Method (MCM)}

Figure III-16 shows the flowchart designed for the propagation of MNFI uncertainties in the state of Durango. This flowchart is like that presented in Section III.1.5.3, except that for the Durango MNFI information is available about the variation in the estimation of the AGB at cluster level according to previous studies. The correlation coefficient between $D B H$ and $T H$ was not used in AGB estimation with $M C M$ because in both periods of the MNFI it was below $|0.08|\left(\rho_{\text {MNFI0409 }}=0.67\right.$ and $\left.\rho_{M N F 10914}=0.65\right)$. The number of iterations used in the $M C M$ was 10,000 times.

\section{III.3.10 Information analysis}

\section{III.3.10.1 General statistics of the study area}

The errors detected when debugging the database of the two MNFI periods analyzed (2004-2009 and 2009-2014), were described. AGB and basal area statistics were analyzed for the two periods, estimated by $D B H$ and $T H$ classes. The analysis included statistics for the two main genera in the study area, Pinus spp and Quercus spp.

\section{III.3.10.2 Reference information to comparison}

Two MNFI periods were analyzed in Durango, the first measurement of 2004-2009 and the first remeasurement 2009-2014. In both cases, field measurements of the variables required for the estimation of AGB ( $D B H, T H$, crown diameter, and wood density) were used. With this information and the allometric models, the AGB was estimated without considering the uncertainty in the calculation, having two reference datasets, 2004-2009 and 2009-2014 for the study area.

\section{III.3.10.3 Error propagation}

Using the $M C M$ and following the flowchart in Figure III-16, the uncertainties were estimated individually for each uncertainty source $(\mathrm{DBH}, \mathrm{TH}$, allometric model and sampling) in the AGB estimate for the MNFI in Durango. This procedure was applied to the two MNFI datasets of this study.

Second uncertainty propagation in AGB estimation was made to evaluate the experience for the field teams as one potential factor for measurement errors. Base data were taken from the measurement errors by the field teams with different experience in forest measurements as described in Section III.2.2.2). The uncertainties of allometric models and cluster design were also included to calculate the propagation of the uncertainty. 
The propagation of uncertainty in the AGB estimate was performed for three levels of vegetation aggregation: ecosystem (temperate forest), stratum (conifers, mixed and oak) and substratum, which divides each stratum into primary and secondary vegetation.

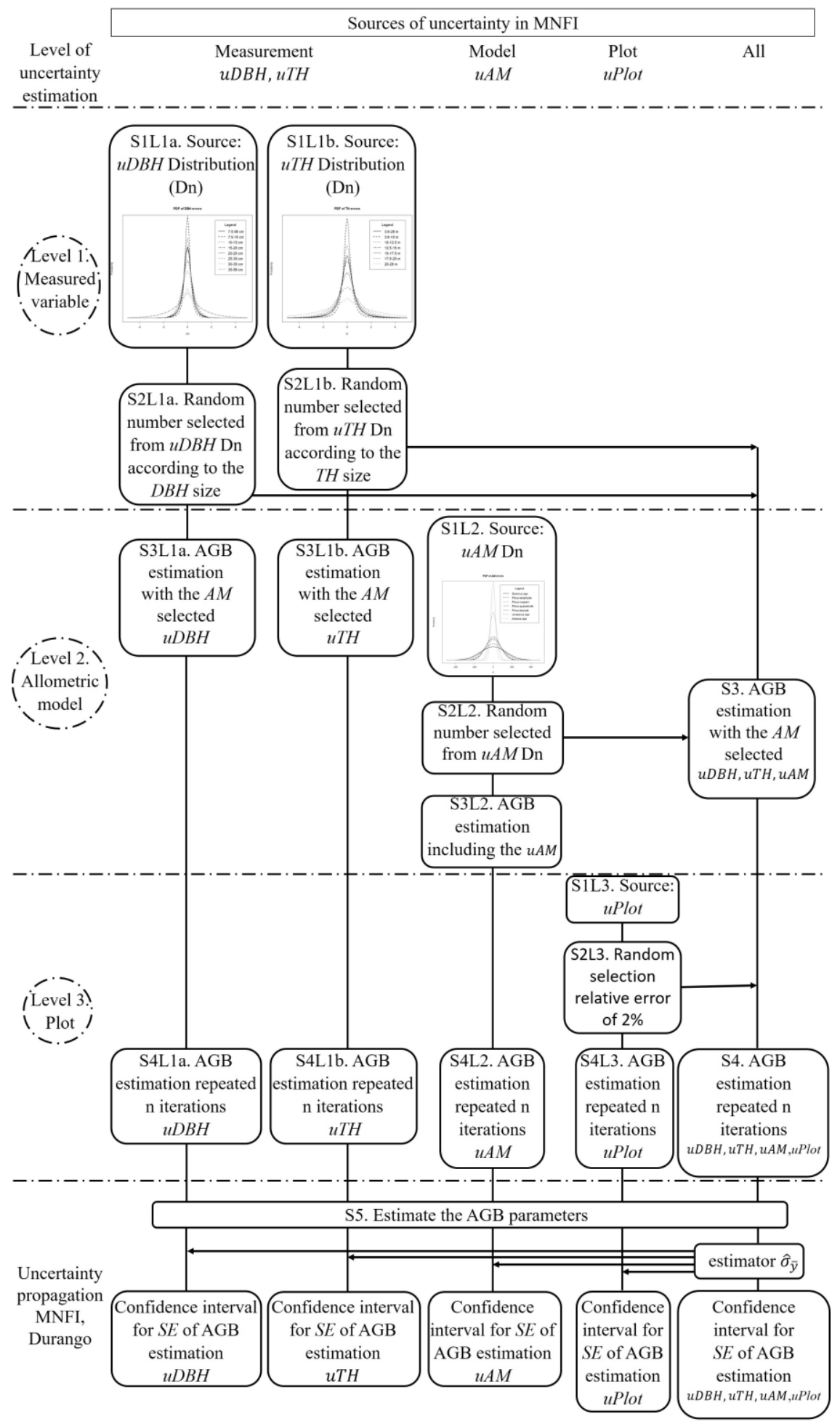

Figure III-16. Flowchart for error propagation of measurement, model and plot design uncertainties for MNFI in Durango, Mexico, using the MCM. 


\section{III.4 Empirical study 4: Modelling and mapping AGB for the state of Durango}

\section{III.4.1 Overview}

In this case study, the AGB was modeled with Landsat imagery, using AGB information calculated from MNFI field measurements. The flowchart in Figure III-17 describes the steps followed in this study, explained in the following lines.

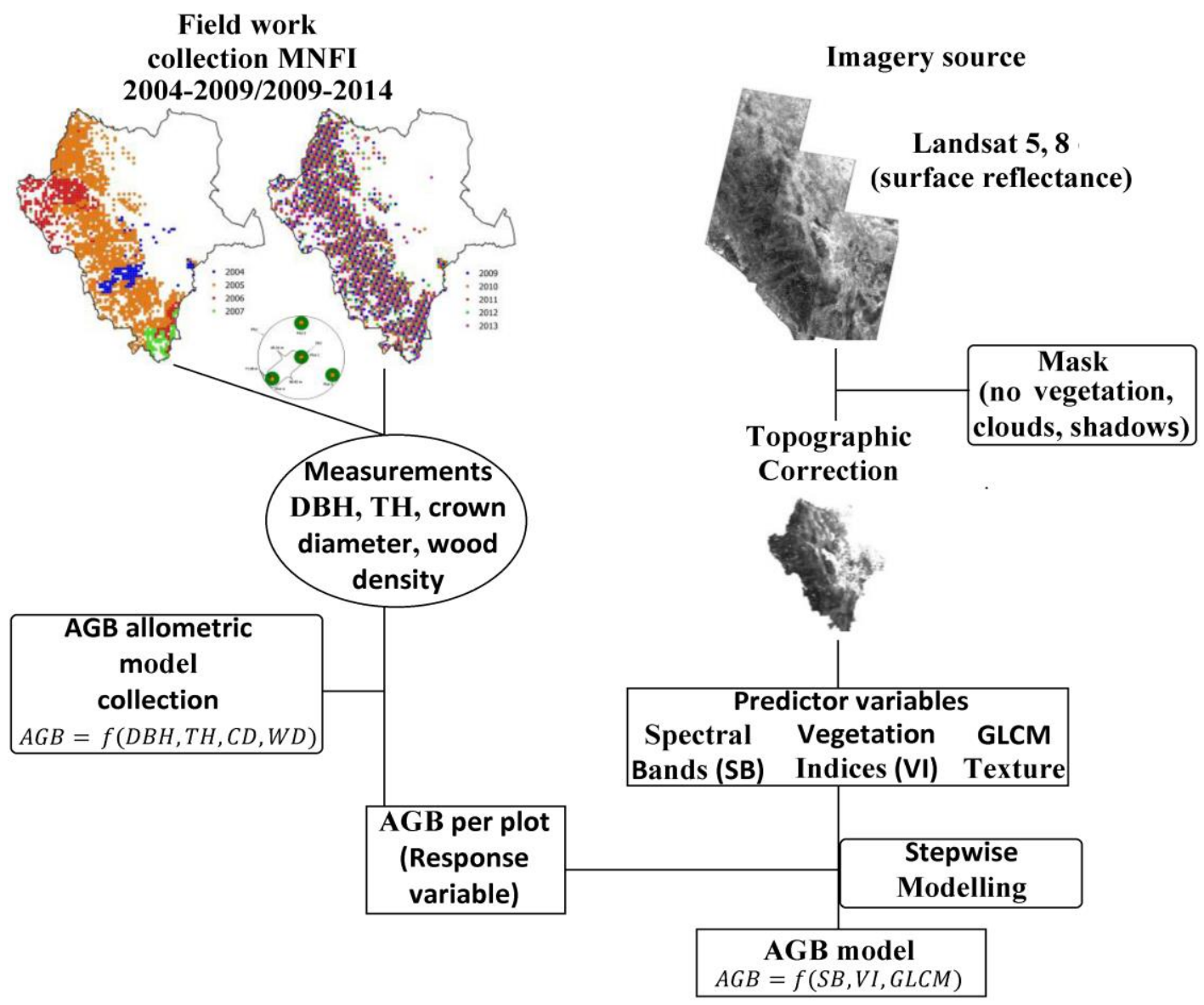

Figure III-17. AGB estimation and mappaing flowchart.

\section{III.4.2 Field data}

Field information from MNFI was registered into different levels of aggregation according to the vegetation type (Table III-9). The information was provided by fieldwork of MNFI and the vegetation series the Institut of statistic and geography in Mexico (INEGI) elaborated from Landsat satellite imagery interpretation (INEGI, 2017).

\section{III.4.3 Remote sensing data processing}

The satellite images used in this study to estimate field measurements were Landsat $5 \mathrm{TM}$ and Landsat 8 OLI, for the two periods analyzed in this study. Scenes LT1 (standard level-one terraincorrected) in surface reflectance values and less than $20 \%$ cloud coverage were requested from the website of the United States Geological Survey (https://earthexplorer.usgs.gov/). The temperate forest of Durango State is covered by eight images (Figure II-4, Section II.2.1) of Landsat 5 from 
7th to 30th April 2007 and Landsat 8 from 28th April to 23th May 2013. The surface reflectance product was orthorectified to WGS84 datum, geometrically calibrated (Wu et al., 2016) and consistently georegistered with $R M S E<6 \mathrm{~m}$ (metadata information) when the average $R M S E$ is $\leq 12$ $\mathrm{m}$ (Young et al., 2017). This product included the visible, near infrared (NIR) and short-wave infrared (SWIR) bands for all the scenes.

\begin{tabular}{ccl}
\hline \multirow{2}{*}{ MNFI } & \multicolumn{2}{c}{ Vegetation Aggregation Level } \\
\cline { 2 - 3 } 2004-2009 & Temperate forest, 1662 Clusters & \multicolumn{1}{c}{ Strata } \\
\hline \multirow{2}{*}{$2009-2014$} & $\begin{array}{l}\text { Conifer forest, 292 Clusters } \\
\text { Mixed forest, 1033 Clusters } \\
\text { Oak forest, 337 Clusters }\end{array}$ \\
\hline & Temperate forest, 1635 Clusters & $\begin{array}{l}\text { Conifer forest, 216 Clusters } \\
\text { Mixed forest, 1156 Clusters } \\
\text { Oak forest, 263 Clusters }\end{array}$ \\
\hline
\end{tabular}

Table III-9. Aggregation levels used to classify vegetation with the number of clusters recorded in the MNFI (CONAFOR, 2012c).

The area of interest in this study was the temperate forest; therefore, areas of non-forest or have pixels with altered values (clouds) were eliminated. Surface reflectance information included spatial data on cloud size and location. This information was used to eliminate the clouds and their shadows.

Non-forest information was obtained from INEGI vegetation and land-use series (LUVS) (INEGI, 2017), using data from anthropogenic use (INEGI, 2014). The fieldwork of LUV Series IV took place between 2007 and 2008, the anthropogenic layer information of this series was used with the Landsat 5 TM imagery (2007). For the information of Landsat 8 OLI (2013), Series V was used which had fieldwork during 2012-2013.

With the cloud's information and the anthropogenic layers of the LUVS, a mask was made to cut the non-forest information and pixels with altered data of Landsat imagery in both periods. Pimple et al. (2017) recommend this procedure prior to topographic correction.

To minimize the shadow effect on the calculation of the indexes to be used to estimate AGB, the C correction method proposed by Teillet et al. (1982) was performed on Landsat images in QGIS (QGIS Development Team, 2019) with the SAGA processing tool. This method has been used in previous studies (Pimple et al., 2017; Riaño et al., 2003; Vázquez-Jiménez et al., Novillo, 2017), recommended for not presenting over-correction (Wu et al., 2016) and reducing the topographic effect in mountain forest terrains (Pimple et al., 2017), such as those of the temperate forest of the state of Durango. The C-correction is defined as follows (Riaño et al., 2003):

$\rho_{H}=\rho_{T}\left(\frac{\cos \theta_{z}+c_{k}}{I L+c_{k}}\right)$ Equation 20

$I L=\cos \theta_{p} \cos \theta_{z}+\sin \theta_{p} \sin \theta_{z} \cos \left(\phi_{a}-\phi_{o}\right) \quad$ Equation 21

Where: $\rho_{H}$ is the reflectance of a horizontal surface, $\rho_{T}$ is the reflectance of an inclined surface, $\mathrm{c}_{\mathrm{k}}=\frac{\mathrm{b}_{\mathrm{k}}}{\mathrm{m}_{\mathrm{k}}}, b_{k}$ is the gradient of regression line for band $k, m_{k}$ is the slope of the regression line for band $\mathrm{k}, b_{k}$ is the intercept in regression line of $\rho_{T}$ vs IL for band k, $m_{k}$ is the slope in regression line of $\rho_{T}$ vs IL for 
band $\mathrm{k}, \theta_{p}$ is the slope angle; $\theta_{z}$ is the solar zenith angle; $\phi_{a}$ is the solar azimuth angle; and $\phi_{o}$ is the aspect angle.

The information source for $\theta_{p}$ and $\phi_{o}$ was the Digital Elevation Model (DEM) version 3.0 (MSE $\leq$ $4.9 \mathrm{~m}$ ) provided by INEGI with spatial resolution of $15 \mathrm{~m}$ scaled to $30 \mathrm{~m}$

(http://www.beta.inegi.org.mx/app/geo2/elevacionesmex/). The values for $\theta_{z}$ and $\phi_{a}$ were obtained from metadata information provided by USGS for every scene, and it was applied for the estimation of $\rho_{H}$ to each band.

With the images corrected, the eight scenes were merged into a mosaic for each spectral band. The scenes were placed in the same hierarchical order and in this way the following calculation of the vegetation indexes was calculated with the corresponding ordered information.

\section{III.4.4 Response variables}

The information of interest for this case study were calculated using the measured variables of MNFI. The response variables used were AGB and basal area $(G)$. Basal area with the $D B H$ measurement was directly obtained and reported in $\mathrm{m}^{2} \mathrm{ha}^{-1}$. AGB calculation was made by tree with 36 allometric models by genus and group of species (Table VIII-1, Appendix I) and reported in $\mathrm{Mg}$ $\mathrm{ha}^{-1}$. The ratio estimators used to calculate the response variables, referred directly to the plot area that was sampled (CONAFOR, 2012c) (Section III.3.5).

\section{III.4.5 Co-registration of remote senting and field plots}

The predictor variables for AGB used in this study were spectral bands, vegetation indexes $(V I)$ and Gray Level Co-occurrence Matrix (GLCM) based texture, calculated from the Landsat imagery for the two data sets used in 2007 and 2013. The spectral bands and VI have been used as predictors of Landsat images to estimate AGB in pine forest (Günlü, et al., 2014), AGB in state inventory for New England, USA (Zheng, Heath, \& Ducey, 2008), among other studies. Lu, 2006 and RodríguezVeiga et al. (2017) emphasized the importance of including other variables to avoid saturation in the AGB estimation when $V I$ are used. Using GLCMs as predictor variables, the AGB estimates have been above the saturation value that is estimated with the use of VI (Kelsey \& Neff, 2014; Wu et al., 2016; Zhao et al., 2016).

\section{III.4.5.1 Spectral Bands}

The spectral bands used in this study were the visible spectrum, NIR and SWIR. The MNFI field manual states that the location of the plots was recorded with an accuracy of up to $15 \mathrm{~m}$, and the coregistration of the Landsat images had an RMSE of less than $6 \mathrm{~m}$, which meant that the values of the estimated AGB could converge on different adjacent pixels of the Landsat image. Therefore, to solve the location issue, a window of $3 \times 3$ pixels was applied to calculate the mean value by pixel of the spectral bands to be used as predictor variables of AGB (A. Günlü et al., 2014; Wu et al., 2016).

\section{III.4.5.2 Vegetation indexes}

Spectral indexes are combinations of spectral reflectance of two or more wavelengths (spectral bands) indicating the relative abundance or accumulation in satellite imagery that can be associated 
with a feature of interest (Bramhe et al., 2018), such as the AGB in this study. Vegetation indexes $(V I)$ are the most popular type that detects the photosynthetic activity of vegetation and are sensitive to AGB estimation (Rodríguez-Veiga et al., 2017).

The NDVI is the most commonly used index for vegetation studies because it is sensitive to the photosynthetically active biomass (Bannari et al., 1995). EVI, WDRVI, and NDMI were calculated as they are used as an alternative to NDVI because they are more sensitive in areas with high AGB and AGB content in tree crowns (Glenn et al., 2008; Henebry et al., 2004; USGS, 2017). SR and $S R G$ were also calculated due they are sensitive to the amount of vegetation and reduce the effect of atmosphere and topography (Glenn et al., 2008). Other indexes such as SAVI, MSAVI, and SATVI have been calculated because they incorporate a correction factor for areas with spaces between vegetation or senescent vegetation, reducing the effect of the soil and dead wood on the collected vegetation information (Marsett et al., 2006; Qi et al., 1994). The calculated VIs are shown in Table III-10, they were calculated using the Grass module of the QGIS program.

\begin{tabular}{|c|c|c|c|}
\hline Index & Calculation & Range & Reference \\
\hline $\begin{array}{l}\text { Atmospherically } \\
\text { Resistant Vegetation }\end{array}$ & $A R V I(A R)=\frac{N I R-R e d-2(\text { Red }- \text { Blue })}{N I R+R e d-2(\text { Red }-B l u e)}$ & -1 to 1 & (Kaufman \& Tanré, 1992) \\
\hline Enhanced vegetation & $E V I(E)=2.5 \frac{N I R-R e d}{(\text { NIR }+6 * \text { Red }-7.5 * \text { Blue })+1}$ & -1 to 1 & (Glenn et al., 2008) \\
\hline $\begin{array}{l}\text { Modified Soil } \\
\text { Adjusted Vegetation }\end{array}$ & $M S A V I(M S A)=\frac{2 * N I R+1-\sqrt{(2 * N I R+1)^{2}-8 *(N I R-R e d)}}{2}$ & -1 to 1 & (Qi et al., 1994) \\
\hline $\begin{array}{l}\text { Normalized } \\
\text { Difference Moisture }\end{array}$ & $N D M I=\frac{N I R-S W I R 1}{N I R+S W I R 1}$ & -1 to 1 & (Wilson \& Sader, 2002) \\
\hline $\begin{array}{l}\text { Normalized } \\
\text { Difference Vegetation }\end{array}$ & $N D V I(N D)=\frac{N I R-R e d}{N I R+R e d}$ & -1 to 1 & (Qi et al., 1994) \\
\hline Simple Ratio & $S R=\frac{N I R}{R e d}$ & 0 to $>30$ & (Glenn et al., 2008; Le \\
\hline Simple Ratio Green & $S R G=\frac{N I R}{\text { Green }}$ & 0 to $>30$ & Maire et al., 2004) \\
\hline $\begin{array}{l}\text { Soil-adjusted Total } \\
\text { Vegetation }\end{array}$ & $S A T V I(S A T)=\frac{S W I R 1-R e d}{S W I R 1+R e d+0.5}(1-0.5)-\frac{S W I R 2}{2}$ & -1 to 1 & (Marsett et al., 2006) \\
\hline $\begin{array}{l}\text { Soil-adjusted } \\
\text { Vegetation }\end{array}$ & $S A V I(S A)=\frac{N I R-R e d}{N I R+R e d+0.5}(1+0.5)$ & -1 to 1 & (Jackson \& Huete, 1991) \\
\hline $\begin{array}{l}\text { Wide Dynamic } \\
\text { Range Vegetation }\end{array}$ & $W D R V I(W D R)=\frac{0.1 *(N I R-R e d)}{0.1 *(N I R+R e d)}$ & -1 to 1 & (Gitelson, 2004) \\
\hline
\end{tabular}

Table III-10. Vegetation indexes evaluated in this study based on spectral bands information of Landsat imagery.

\section{III.4.5.3 Textures}

The texture is a metric of pixel variability across neighboring pixels for a defined processing window (Kelsey \& Neff, 2014). GLCM-based texture measurements provides the basis for calculating multiple first or second order statistical quantities and were defined by Haralick et al. (1973), currently the common procedure for obtaining texture from images (Zhao et al., 2016). Based on AGB estimates in studies conducted in temperate and subtropical forests (Safari \& 
Sohrabi, 2016; Wu et al., 2016; Zhao et al., 2016) the textures to be extracted from Landsat images were selected (Table III-11).

Texture has been calculated for spectral bands (Fuchs et al., 2009; Kelsey \& Neff, 2014; Safari \& Sohrabi, 2016; Wu et al., 2016) and for vegetation indexes (Lopez-Serrano et al., 2015). In this study, a Pearson correlation was performed between the response variables (AGB and basal area) and the predictor variables (spectral bands and vegetation indexes). From the two periods of MNFI were selected the predictor variables with the highest correlation coefficient Table III-12. In these response variables were calculated the Haralick textures.

\begin{tabular}{|c|c|c|c|}
\hline Feature extracted & Calculation & Feature extracted & Calculation \\
\hline Mean $(M N)$ & $\sum_{i, j=0}^{N-1} i P_{i, j}$ & Dissimilarity $(D I)$ & $\sum_{i, j=0}^{N-1} i P_{i, j}|i-j|$ \\
\hline Variance $(V A)$ & $\sum_{i, j=0}^{N-1} i P_{i, j}(i-M E)^{2}$ & Entropy $(E N)$ & $\sum_{i, j=0}^{N-1} i P_{i, j}\left(-\ln P_{i, j}\right)$ \\
\hline $\begin{array}{l}\text { Haralicks } \\
\text { correlation }(H C)\end{array}$ & $\sum_{i, j=0}^{N-1} \frac{P_{i, j}-M E^{2}}{V A}$ & $\begin{array}{l}\text { Energy or Angular } \\
\text { Second Moment }(A S M)\end{array}$ & $\sum_{i, j=0}^{N-1} P_{i, j}^{2}$ \\
\hline Correlation $(C R)$ & $\sum_{i, j=0}^{N-1} \frac{P_{i, j}(i-\mu)(j-\mu)}{\sqrt{\left(\sigma_{i}^{2}\right)\left(\sigma_{j}^{2}\right)}}$ & $\begin{array}{l}\text { Inverse different moment } \\
\text { or homogeneity }(\mathrm{HO})\end{array}$ & $\sum_{i, j=0}^{N-1} i \frac{P_{i, j}}{1+(i-j)^{2}}$ \\
\hline Contrast $(C O)$ & $\sum_{i, j=0}^{N-1} i P_{i, j}(i-j)^{2}$ & & \\
\hline
\end{tabular}

Table III-11. Texture variables used. $P(i, j)$ is the normalized co-occurrence matrix such that sum $(i, j=0, N-1)(P(i, j))=1$ (Haralick et al., 1973).

\begin{tabular}{|c|c|c|c|c|c|}
\hline \multicolumn{3}{|c|}{ Landsat 5 (2007) } & \multicolumn{3}{|c|}{ Landsat 8 (2013) } \\
\hline & $G$ & $W$ & & $G$ & $W$ \\
\hline$A R V I$ & $0.77 * * *$ & $0.72 * * *$ & $A R V I$ & $0.75 * * *$ & 0.76 **** \\
\hline$N D V I$ & $0.79 * * *$ & $0.73 * * *$ & $N D V I$ & $0.75^{* * *}$ & $0.76 * * *$ \\
\hline$S R G$ & $0.79 * * *$ & $0.74 * * *$ & $S R$ & $0.72 * * *$ & $0.76 * * *$ \\
\hline WDRVI & $0.79 * * *$ & $0.74 * * *$ & WDRVI & $0.74 * * *$ & $0.77 * * *$ \\
\hline
\end{tabular}

Table III-12. Vegetation indexes with the highest Pearson correlation coefficient, selected to apply on them the texture calculation.

To estimate the texture it was necessary to define the size of the window to calculate the GLCM (Bramhe et al., 2018). The window size should be appropriate so that the variation will not be exaggerated neither there will be an excess of smoothing in the variation, small and large window size, respectively (Dengsheng Lu, 2006).

In subtropical forest, (Wu et al., 2016) using a window of 3 x 3 pixels for Landsat imagery, detected changes in AGB storage in a 10 years period study. Attarchi and Gloaguen (2014), found a higher correlation in AGB estimation with textures in window size of 11X11 pixel for temperate forest with Landsat images, in comparison of AGB estimation to vegetation indeces. (Kelsey \& Neff, 2014), implementing different window sizes ( $3 X 3,5 X 5,7 X 7$ and 9X9), found that $3 X 3$ is the optimal size for estimating AGB in temperate forest. Similarly, (Lopez-Serrano et al., 2015) tested 
three window sizes $(3 \mathrm{X} 3,5 \mathrm{X} 5$ and $7 \mathrm{X} 7)$ in temperate forest, finding that the combination of the texture variables and window size are important to optimize mixed models for estimating AGB in Landsat images, not concluding in an optimal window size using texture for AGB estimation. In this study, and according to previous estudies, the textures were calculated for three window sizes $3 \mathrm{X} 3$ (Kelsey \& Neff, 2014), 7 X7 (P. López-Serrano et al., 2015) and 11X11(Attarchi \& Gloaguen, 2014). The extraction of the textures from the satellite imagery was made using the Orfeo Tool Box (OTB) module implemented in QGis.

\section{III.4.6 AGB models assessment and validation}

AGB and basal area, as determined in the field plots, were modelled from the remote sensing data with predictor variables as of Table III-13. For the spectral bands and for the vegetation indexes, a $3 \mathrm{X} 3$ window was applied to calculate the mean value by pixel (see Section III.4.5.1).

\begin{tabular}{|c|c|}
\hline Landsat 5 (2007) & Landsat 8 (2013) \\
\hline Spectral bands (6) & Spectral bands (7) \\
\hline$R, G, B, N I R, S W I R 1, S W I R 2$ & Coastal, R, G, B, NIR, SWIR1, SWIR2 \\
\hline Vegetation Indexes (10) & Vegetation Indexes (10) \\
\hline $\begin{array}{l}A R V I, E V I, M S A V I, N D M I, N D V I, S R, S R G, S A T V I, S A V I \text {, } \\
\text { WDRVI }\end{array}$ & $\begin{array}{l}\text { ARVI, EVI, MSAVI, NDMI, NDVI, SR, SRG, SATVI, SAVI, } \\
\text { WDRVI }\end{array}$ \\
\hline Texture metrics (243) & Texture metrics (243) \\
\hline $\begin{array}{l}\text {-9 Image: Red, Green, Blue, NIR, ARVI, NDVI, SR, SRG, } \\
\text { WDRVI }\end{array}$ & $\begin{array}{l}\text {-9 Image: Red, Green, Blue, NIR, ARVI, NDVI, SR, SRG, } \\
\text { WDRVI }\end{array}$ \\
\hline -3 Kernel size: $3 X 3(3), 7 X 7(7), 11 X 11(11)$ & -3 Kernel size: $3 X 3(3), 7 X 7(7), 11 X 11(11)$ \\
\hline $\begin{array}{l}-9 \text { Haralick texture: } M N, V A, H C, C R, C O, D I, E N, A S M \text {, } \\
H O\end{array}$ & $\begin{array}{l}-9 \text { Haralick texture: } M N, V A, H C, C R, C O, D I, E N, A S M \text {, } \\
H O\end{array}$ \\
\hline 259 variables & 260 variables \\
\hline
\end{tabular}

Table III-13. Landsat image predictor variables $(P V)$ used to evaluate models for estimating AGB and basal area.

The texture metrics were constructed using the image with the spectral band information or VI, three window sizes and the Haralick texture estimation. Figure III-18 shows the construction of the texture metrics.

The Box-Cox transformation (Box \& Cox, 1964) was applied to the response variables using the package $\mathrm{R}$ ( $\mathrm{R}$ Core Team, 2018). This transformation corrects biases in the distribution of errors, also unequal variances and mainly non-linearity in the relation of predictor variable with the response variable (Box \& Cox, 1964). The exponent Lambda $(\lambda)$ was the central part of the transformation of Box-Cox. The transformation of y (response variable) has the following form (Box \& Cox, 1964): 
Image (Bands and $V I$ )

Red (Rd), Green (G), Blue (B), NIR (N), ARVI (AR),

NDVI (ND), SR, SRG, WDRVI (WDR)

Kernel size (window)

3X3 (3), 7X7 (7), 11X11 (11)

Metrics (Haralick texture)

Mean (MN), Variance (VA), Haralick correlation (HC), correlation (CR), Contrast (CO), Dissimilarity (DI), Entropy (EN), Angular Second Moment (ASM), Homogeneity (HO)
Texture Metric:

Haralicks correlation

estimated in window $11 X 11$

of the Red band image

Figure III-18. Texture metrics construction with the image (spectral band or vegetation index), window size (in pixels) and the Haralik texture estimate.

$y(\lambda)= \begin{cases}\frac{y^{\lambda}-1}{\lambda}, & \text { if } \lambda \neq 0 ; \\ \log y, & \text { if } \lambda=0 .\end{cases}$

Equation 22

The values of $\lambda$ were the optimal value to correct the data and its value varied from -5 to 5 . The optimal value of $\lambda$ gave the best approximation of a normal distribution curve (Box \& Cox, 1964;

García-Pérez, 2014).

The transformed response variables were modeled with the Landsat imagery data using a stepwise multiple regression analysis (Fuchs et al., 2009; A. Günlü et al., 2014; Ou et al., 2019; Safari \& Sohrabi, 2016; Wu et al., 2016). A stepwise regression analysis was done with the statistics software R using the MASS package (R Core Team, 2018). A standard model was used:

$y_{i}=\beta_{0}+\beta_{1} x_{1}+\beta_{2} x_{2}+\ldots .+\beta_{n} x_{n}+\varepsilon$

Equation 23

Where: $y_{i}$ were the response variables (AGB or basal area); $x_{n}$ are the predictor variables; $\beta_{n}$ are the parametric regression coefficients to be estimated; $\varepsilon$ is the error of the model.

This model was used to estimate the AGB based on four vegetation types according to the vegetation aggregation level (Table III.9). Models selected for each level of aggregation were those with the highest coefficient of determination $\left(R^{2}\right)$ and significance in of the predictor variables $\mathrm{p}$ value $<0.05$. To estimate multicollinearity of the predictor variables (A. Günlü et al., 2014), for each model the variance inflation factor (VIF) was calculated through the mctest package in the program R (R Core Team, 2018). Using the VIF criterion proposed by (Marquardt, 1970), the models without multicolliniarity were selected.

Repeated K-fold cross validation was the method used to evaluate the model (Langford, 2005), splitting the data into $\mathrm{K}$ sections where the model was evaluated and selecting the number of times the process was repeated; the precision of the model ( $R M S E$ ) was the average of the repetitions executed. The validation was performed with the caret package in program R (R Core Team, 2018). The evaluation of the models included the estimation of the absolute and relative bias in the data estimated by the model (with Landsat predictor variables) compared to the data measured in the MNFI (Fuchs et al., 2009). Bias estimators were obtained using the following equations:

Absolute Bias $=\frac{1}{\mathrm{n}} \sum_{\mathrm{n}=1}^{\mathrm{n}}\left(\widehat{\mathrm{x}}_{\mathrm{i}}-\mathrm{x}_{\mathrm{i}}\right)$

Equation 24 
Relative Bias $=\left(\operatorname{Bias}_{\mathrm{r}}\right)$ in $\%=\frac{\text { Bias }}{\overline{\mathrm{x}}} * 100 \quad$ Equation 25

Where: $\widehat{x}_{i}$ were the estimated values from the model adjusted (AGB, basal area); $x_{i}$ were the observed values from MNFI (AGB, basal area); $\bar{x}$ was observed mean value (AGB, basal area).

After validation, the models were used to produce maps for the response variables (AGB and basal area). 


\section{Chapter IV: Results}

\section{IV.1 Empirical study 1: Double measurement of dasometric variables to estimate the measurement uncertainty and error propagation of aboveground biomass estimation in pine forests}

\section{IV.1.1 General information}

In this study, 4292 trees in the 10 plots were measured. During the fieldwork, a control of the measurements was made and 34 writing errors and 35 inconsistency errors ( $D B H$ and $T H$ relationship) were corrected. In addition, the field records were verified, and it was found that 30 trees were measured only once; these were not used in the estimation of discrepancies. Thus, the number of trees with double measurement was 4262 .

The species Pinus cooperi dominated, spanning $92.8 \%$ of the trees sampled. Table IV-1 shows the mean and range of values from measured and estimated variables. Figure IV-1 shows the perhectare basal area and AGB estimates both based upon $D B H$ class and $T H$ for the trees sampled.

\begin{tabular}{lccccc}
\hline $\begin{array}{l}\text { Variable / } \\
\text { estimate }\end{array}$ & $\begin{array}{c}\text { Tree } \\
\text { No. ha }\end{array}$ & $\begin{array}{c}D B H \\
(\mathrm{~cm})\end{array}$ & $\begin{array}{c}\text { TH } \\
(\mathrm{m})\end{array}$ & $\begin{array}{c}G \\
\left(\mathrm{~m}^{2} \mathrm{ha}^{-1}\right)\end{array}$ & $\begin{array}{c}W \\
\left(\mathrm{Mg} \mathrm{ha}^{-1}\right)\end{array}$ \\
\hline $\begin{array}{l}\text { Mean value } \\
\text { Range }\end{array}$ & 1137 & 16.9 & 13.3 & 29.17 & 176.07 \\
\hline
\end{tabular}

Table IV-1. Range and mean values of the measured and estimated variables in the pine forests of El Salto, Durango.

Figure IV-1 shows that $95 \%$ of the trees had $D B H \leq 30 \mathrm{~cm}$ and that the $D B H$ class with the largest number of trees was (10-15] cm. The $D B H$ class with the greatest contribution in the basal area and AGB was $(15,20] \mathrm{cm}$, was also the class of the overall mean $D B H$ of $16.9 \mathrm{~cm}$. On the other hand, trees over $30 \mathrm{~cm} D B H$ represented $4.5 \%$ of the sample. However, they contributed to $16.5 \%$ of the basal area and $20.2 \%$ of the AGB.

The overall $\mathrm{TH}$ mean was $13.3 \mathrm{~m}$; from the dataset, $90 \%$ of the trees measured were less than 17.5 $\mathrm{m}$ and the class (12.5-15] m contained the most trees. The $T H$ class with the highest contribution to AGB and basal area was $(15,17.5] \mathrm{m}$. Trees taller than $17.5 \mathrm{~m}$ comprised $9.6 \%$ of the data, although the contribution of these trees was $24.8 \%$ and $30.5 \%$, for basal area and AGB, respectively.

\section{IV.1.2 $\quad D B H$ and $T H$ measurements}

\section{IV.1.2.1 Diameter at breast height}

Analysis of the boxplots from the two datasets (Figure IV-2, left) shows their similarity, with a median value of $15.75 \mathrm{~cm}$ for the first Measurement $(M 1)$ and $15.85 \mathrm{~cm}$ for the re-measurement (M2). The histograms of $M 1$ and $M 2$ were superimposed and found 98.4\% overlap. A high correlation of $\rho_{(M 1, M 2)}=0.9864$ between $M 1$ and $M 2$ was estimated. 


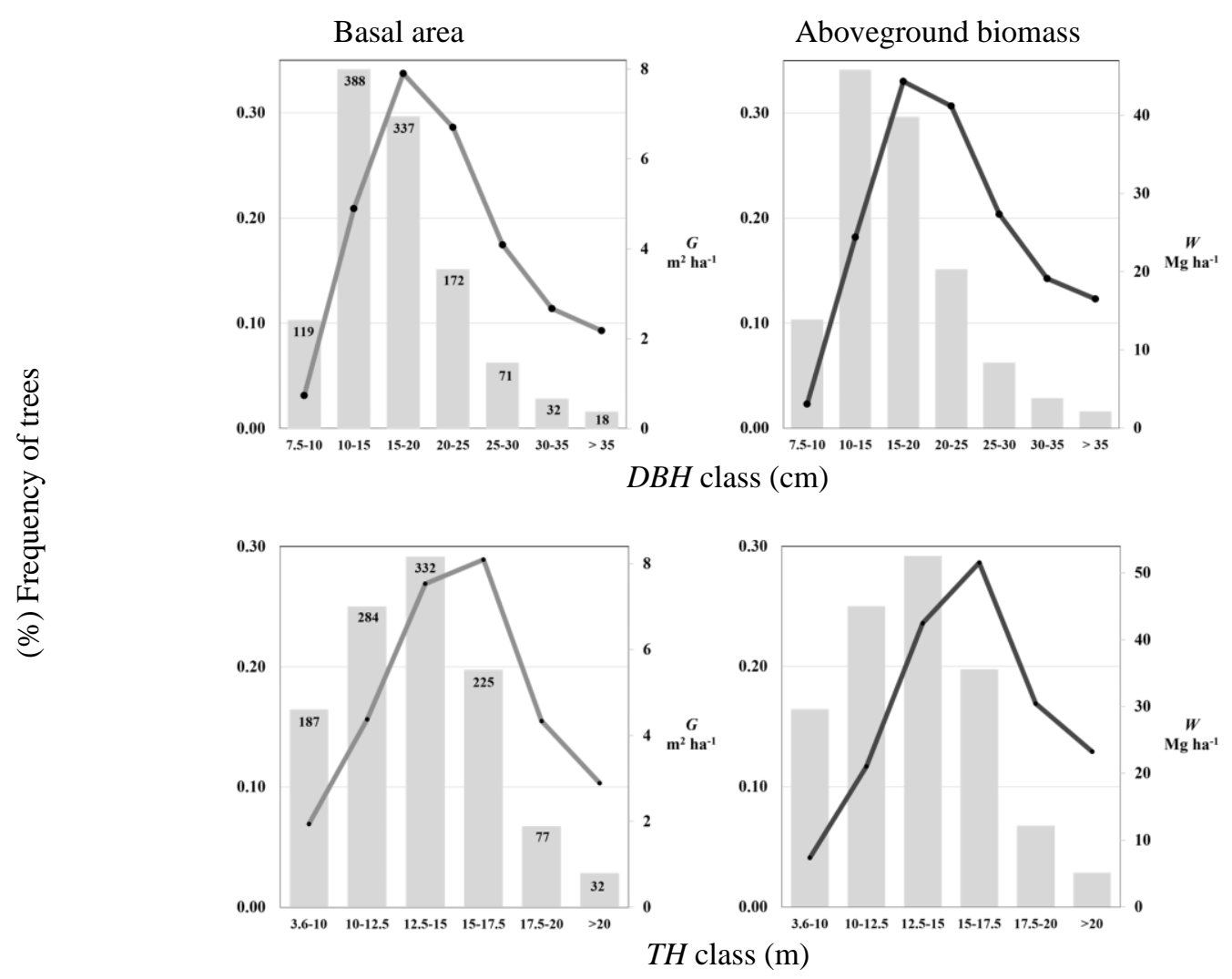

Figure IV-1. Basal area and AGB estimation of the study area. Above, estimate by $D B H$ class every $5 \mathrm{~cm}$; below, estimate by $T H$ class every $2.5 \mathrm{~m}$.
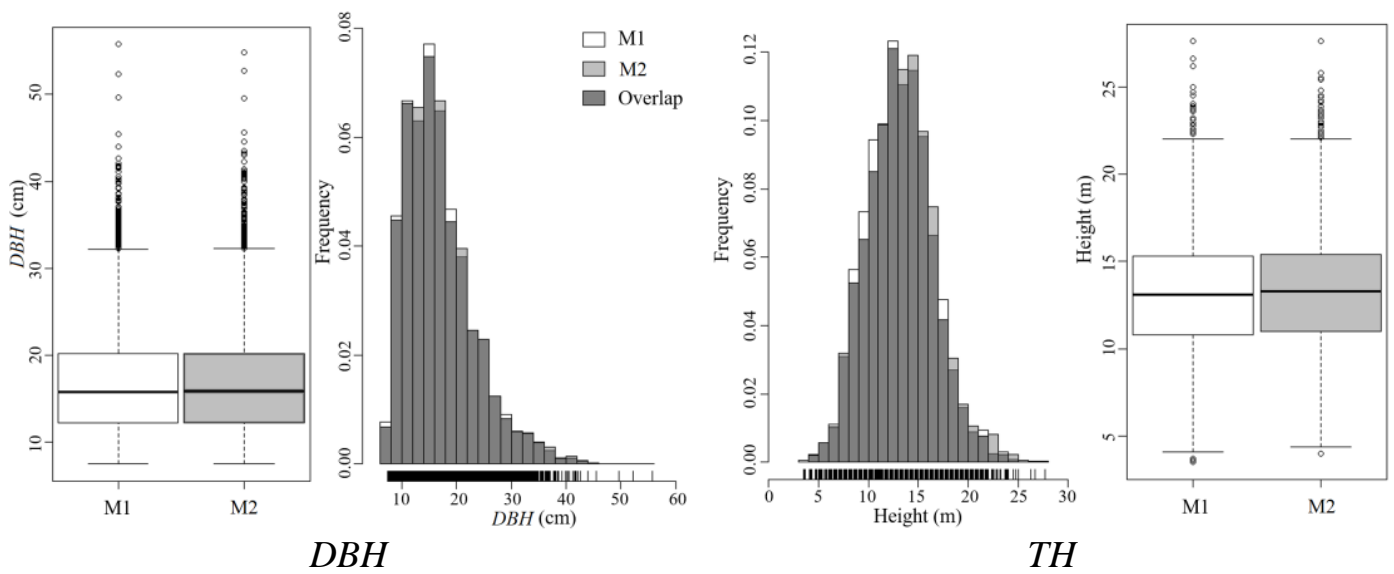

Figure IV-2. Left: $D B H$ data boxplot, histograms of Measurement $(M 1)$ and Re-measurement (M2). Right: a similar case for $T H$. Bottom, the trend line of $M 2$ versus $M 1$ for $D B H$ and $T H$.

The data pairs were compared with non-parametric methods due to the rejection of normality (pvalue $_{\mathrm{KS}}<2.2 \mathrm{e}^{-16}$ ) with the Kolmogorov-Smirnov Test (Lilliefors correction). The variances of the datasets were compared with Bartlett's Test $\left(p-\right.$ value $\left._{B}=0.6671\right)$ and Fligner-Killeen's Test (pvalue $\left._{\mathrm{FK}}=0.9354\right)$, and not significance for variance difference was found. With the Wilcoxon Signed-Rank Test, medians of the datasets were compared. This test showed that the difference 
between medians did not include, meaning that the populations were not identical (p-value $\mathrm{wrt}_{\mathrm{rt}}<2.2 \mathrm{e}^{-}$ $\left.{ }^{16}\right)$.

\section{IV.1.2.2 Total height}

A graphical evaluation was performed, as with the $D B H$ data, for the $T H$ measurements (Figure IV2 , right). The histograms show the areas where frequencies of the dataset overlap (95\%) and the areas where the frequency of one of the sets was most prevalent. The estimation of the median values was $13.17 \mathrm{~m}$ for $M 1$ and $13.35 \mathrm{~m}$ for $M 2$. Data dispersion was greater for $T H$ compared to the $D B H$ measurements, and the correlation coefficient was $\rho_{(M 1, M 2)}=0.9651$.

Normality in data distribution for $M 1$ and $M 2 T H$ measurements was rejected (p-value $\mathrm{KS}_{\mathrm{K}}=0.0001298$ and $\mathrm{p}$-value $\mathrm{KS}_{\mathrm{KS}}<2.088 \mathrm{e}^{-07}$, respectively). The Bartlett and Fligner-Killeen Tests did not reject the homogeneity of the variances $\left(p-\right.$ value $_{\mathrm{B}}=0.3947$ and $p$-value $\mathrm{FK}_{\mathrm{FK}}=0.7991$ ), and the Wilcoxon Signed Rank Test stated that databases do not belong to identical populations ( $\mathrm{p}$-value $\mathrm{W}_{\mathrm{rt}}<2.2 \mathrm{e}^{-16}$ ).

The applied tests showed differences between the data pairs ( $D B H$ and $T H)$. However, because the field manual was followed, it was assumed that the data collection was free of systematic errors. Therefore, the source of these measurement differences was attributed to random error.

\section{IV.1.3 DBH and $\mathrm{TH}$ measurement error}

\section{IV.1.3.1 DBH measurement error}

The $D B H$ measurement errors of the 4262 trees were organized by frequency distribution. The $0.92 \%$ of the measured trees had an error greater than $2 \mathrm{~cm}, 93.24 \%$ of the trees had an error between 0 and $2 \mathrm{~cm}$, and $5.94 \%$ did not show difference between measurements. The mean error was $0.4 \mathrm{~cm}$, and the maximum was $4.6 \mathrm{~cm}$.

As explained in Section III.1.5.1.1.1, the frequency distribution of $D B H$ measurement errors was fitted to a probability density function (PDF) to describe the errors through the parameters of the PDFs. The PDFs were fitted using the whole dataset of errors (Figure IV-3a) and the errors by DBH class (Figure IV-3b to h). See Table VIII-2 of Appendix II for the parameters and goodness-of-fit for the fitted PDF's. Since the GUM Method of error propagation use the variance as the parameter to propagate uncertainty, the dataset of measurement errors was adjusted to a normal distribution (Figure IV-3, gray line distribution). The Monte-Carlo simulation approach (MCM), to propagate the errors, can use any PDF describing those errors. Therefore, the best adjusted PDFs per measurement error class and for the whole data set were used for the MCM (Figure IV-3, dark line distribution).

For the measurement error of the first $D B H$ class, the best PDF was the Scaled-Shifted $t$ distribution. In the following four classes, Johnson's distribution had the best fit for measurement error. In the last two classes of the largest trees, the error followed a pattern of Laplace distribution. The best-fitted distributions differed slightly from the normal distribution for classes of $D B H$ less than $30 \mathrm{~cm}$, even in some classes it was not possible to distinguish the difference because the two distributions overlap. However, in the two classes with larger $D B H$, the difference between the normal distribution and the best-fitted distribution was evident. 

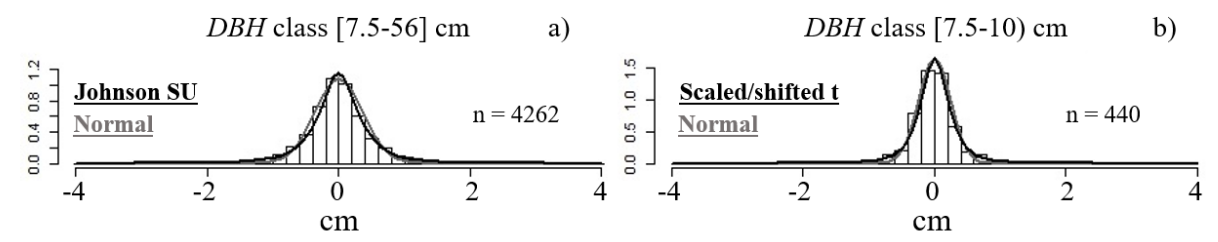

$D B H$ class $[10-15) \mathrm{cm}$

c)

$D B H$ class $[15-20) \mathrm{cm}$

d)

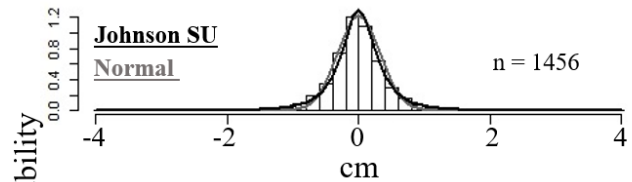

4 -4
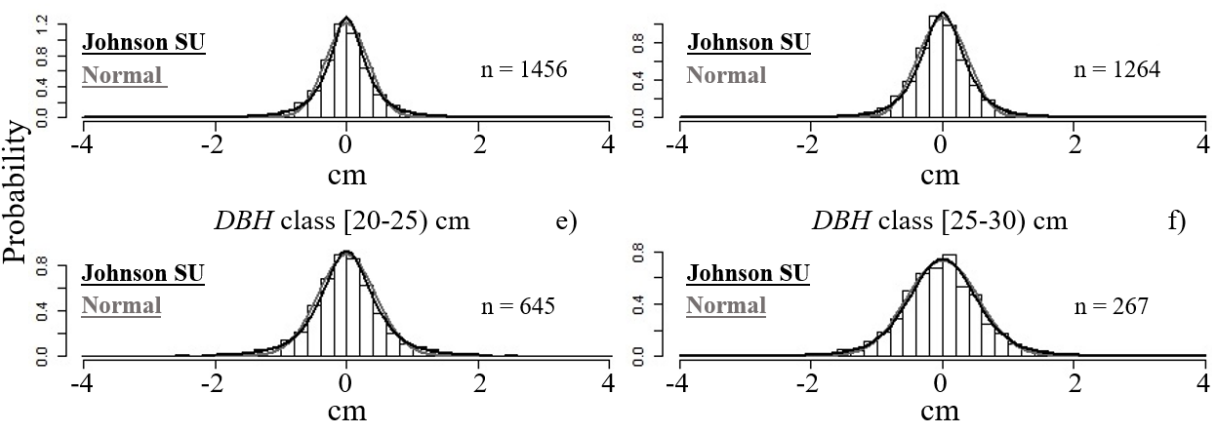

$D B H$ class $[30-35) \mathrm{cm}$

g)

$D B H$ class [35-56] $\mathrm{cm}$

h)

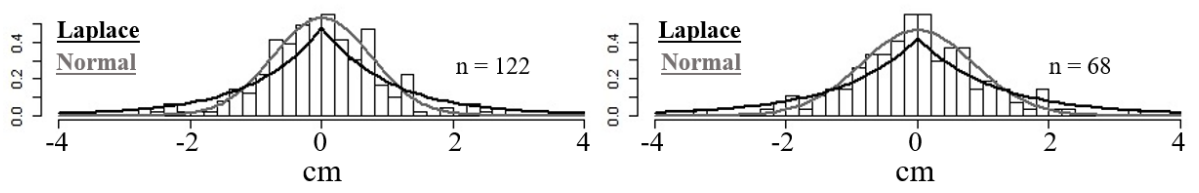

Figure IV-3. Probability Density Functions (PDF) fitted with $D B H$ measurement errors according to $D B H$ class.

\section{IV.1.3.2 TH measurement error}

In $7.88 \%$ of the 4262 trees measured, were not find difference between measurements. The $87.35 \%$ of the trees had errors greater than zero and less than $2 \mathrm{~m}$, and the remaining trees $(4.77 \%)$ had errors between 2 and $4 \mathrm{~m}$. The largest error was $4 \mathrm{~m}$, and the mean $T H$ measurement error was 0.61 $\mathrm{m}$. PDFs for the entire dataset (Figure IV-4a) and by $T H$ classes (Figure IV-4b to g) were adjusted. In each case, the PDF that best adjusted to the distribution of errors (Figure IV-4, dark line distribution), as well as the normal distribution (Figure IV-4, gray line distribution) were used. TH classes less than $17.5 \mathrm{~m}$ followed Johnson's distribution. The last two classes fitted to the ScaledShifted $t$ and Laplace distributions. The parameters, which describe each distribution and the goodness-of-fit, are in Appendix II (Table VIII-3).

\section{IV.1.4 Error propagation}

\section{IV.1.4.1 GUM Method for error propagation}

\section{IV.1.4.1.1 General statements}

The error propagation results using the GUM Method are shown in Table IV-2. This table has two sections (non-sampling and stand-level) summarizing the contributions from sources of uncertainty in the AGB estimation. In the upper part of the table, the contributions at the average tree (treelevel) and stand-level are in the same units of the estimated values, $\mathrm{kg}$ per tree and $\mathrm{Mg}$ per ha. In the lower part, the percentages by each source of uncertainty at tree-level and stand-level were provided. 

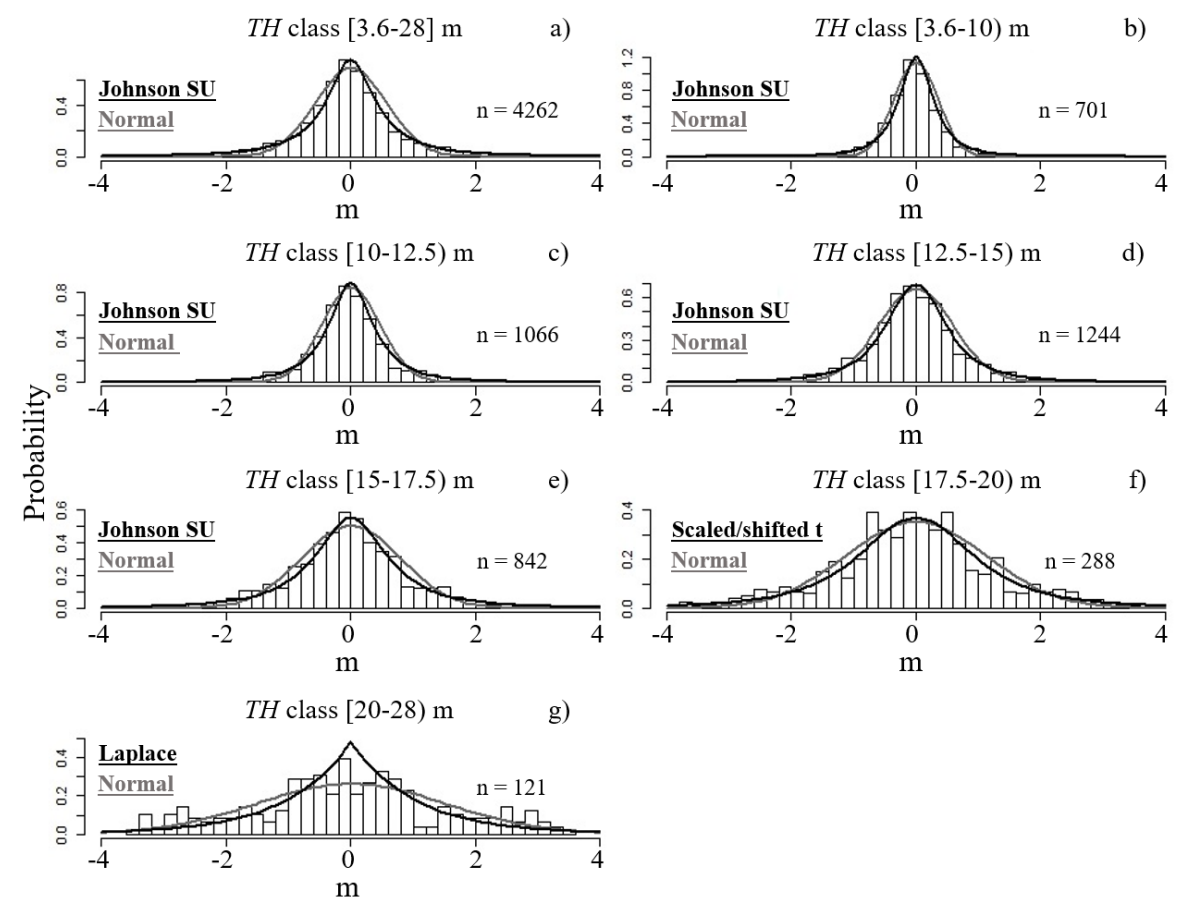

Figure IV-4. PDF fitted with $T H$ measurement error according to $T H$ class.

\section{IV.1.4.1.2 Tree-level uncertainty}

The mean uncertainty contributions to AGB estimation by tree of $D B H$ measurements $(u D B H), T H$ measurements $(u T H)$, and their correlation $\left(u \rho_{(D B H, T H)}\right)$ are shown in Table IV-2.

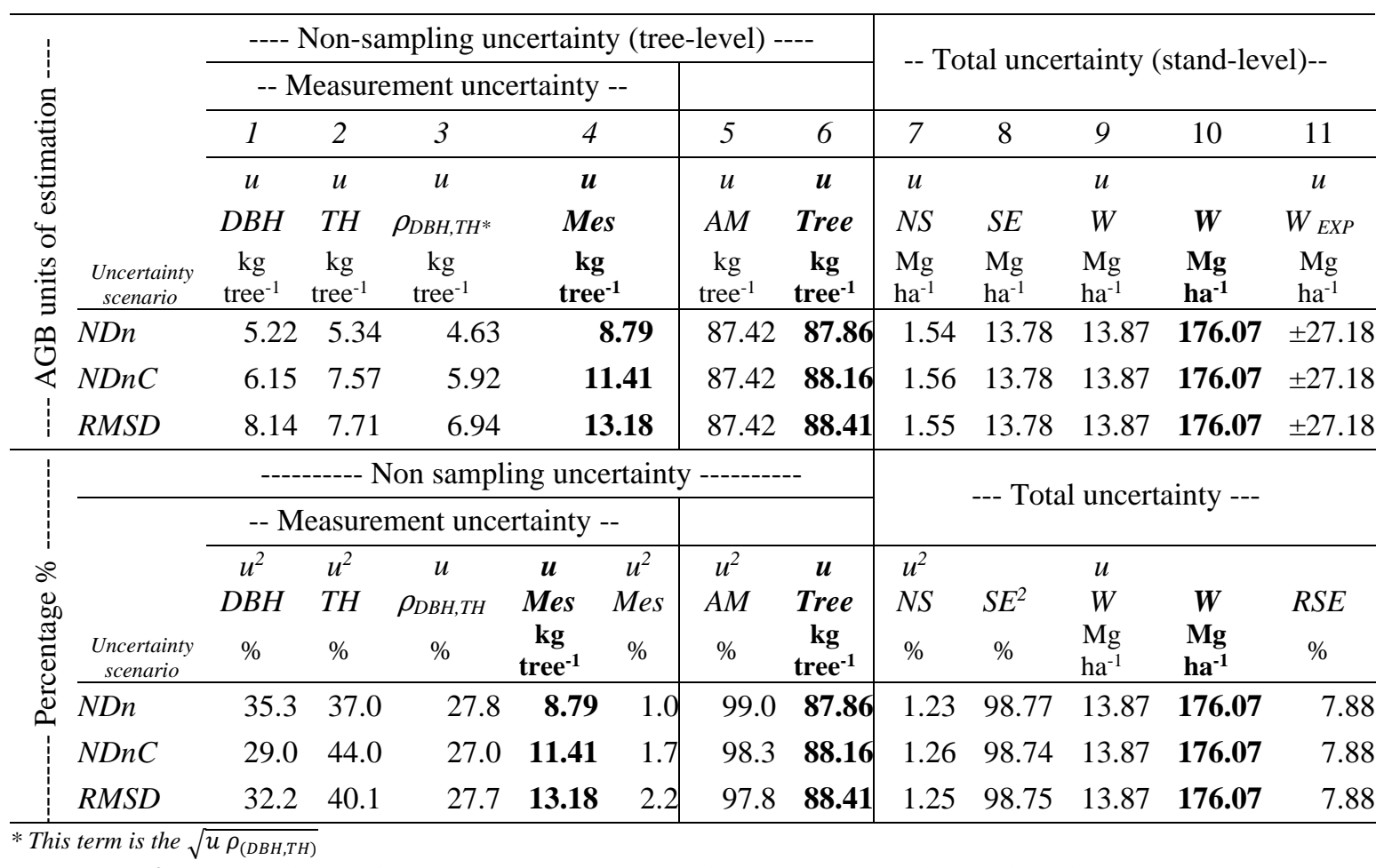

Table IV-2. Uncertainty of the AGB calculation using three scenarios estimating measurement uncertainty $N D n, N D n C$ and $R M S D$. 
The scenarios used to estimate the measurement uncertainty parameter ( $\hat{\sigma}=$ standard deviation) are represented by their acronyms (see Table III-2). The three scenarios were (i) measurement errors fitted to normal distribution ( $N D n$ ), (ii) measurement errors fitted to a normal distribution by $D B H$ class and by $T H$ class $(N D n C)$, and (iii) root mean square deviation of measurement (RMSD). The parameters of the scenarios NDn and NDnC are in Tables VIII-2 and VIII-3 of Appendix II. Parameters of the RMSD scenario were $\hat{\sigma}_{D B H}=0.53 \mathrm{~cm}$ for $D B H$ and $\hat{\sigma}_{T H}=0.89 \mathrm{~m}$ for $T H$.

In the first combined uncertainty (column 4), the scenario with the highest estimation was $R M S D$ with $13.18 \mathrm{~kg}$ per tree, followed by $N D n C$ with $11.41 \mathrm{~kg}$, and finally by the $N D n$ with $8.79 \mathrm{~kg}$. The sources of uncertainty ( $D B H, T H$, and correlation), are shown in the lower part of the table. The largest contribution was made by $u D B H$, followed by $u T H$, and finally $u \rho_{(D B H, T H)}$.

In the lower part of Table IV-2 are the contributions calculated from Equations 06b, 07, and 9. The $u T H$ was the highest contribution for all the scenarios applied with a range between 37 and $44 \%$. The $u D B H$ was the second most important contribution of measurements at tree-level and it ranged from 29 to $35.3 \%$. Finally, the smaller contribution in measurement was the correlation within a short range between $27-27.8 \%$.

Once $u$ Tree was estimated (column 4), the uncertainty of the allometric model ( $u A M$ in column 5) was added to obtain the second combined uncertainty or uncertainty of non-sampling (uTree in column 6), still at the tree-level. The contribution of $u A M$ to $u$ Tree ranged from 97.8 to $99.0 \%$.

\section{IV.1.4.1.3 Total AGB uncertainty}

After the aggregation process from tree-level to plot level, the total non-sampling uncertainty ( $u N S)$ was combined with the sampling error ( $S E$ in column 8 ) to estimate the total uncertainty ( $u W$ in column 9). In this step, the contribution of $u N S$ was similar for all three scenarios, ranging from $1.23-1.26 \%$ of $u W$, with more than $98.9 \%$ contribution due to the $S E$. The last two columns are the average estimate of AGB $(W)$ and the expanded uncertainty ( $u W_{E X P}$, Equation 13). Using Equation 12 with $u W_{E X P}$, the $95 \%$ confidence interval for $W$ was calculated and gave the same result for the three scenarios of 148.89 to $203.25 \mathrm{Mg} \mathrm{ha}^{-1}$. Moreover, the lower part of column 11 shows that the total relative standard error, calculated to be $7.88 \%$, was also the same for the three scenarios.

ANOVA for repeated measurements was applied to compare the mean uncertainty estimate at plotlevel (Tables VIII-8 and VIII-9, Appendix IV). From this analysis, a significant difference (p-value $=0.0422$ ) was found between the means of the different scenarios. Through applying a T-Test with the Holm correction (Table VIII-10, Appendix IV), a significant difference ( $\mathrm{p}$-value $\mathrm{t}_{\mathrm{tt}}=0.0037$ ) was found between the NDn and RMSD scenarios. In contrast, no significant difference was found between the $N D n C$ scenario and the other two scenarios ( $\mathrm{p}$-value ${ }_{\mathrm{tt}}>0.05$ ).

\section{IV.1.4.1.4 Total AGB contribution by source of non-sampling uncertainty}

To estimate the contribution from sources of $u N S$ to the $u W$, the error propagation with the NDnC scenario (normal distribution adjusted by class) was estimated. Table IV-3 shows the results of the error propagation using one uncertainty source at a time, and thus the individual contribution by uncertainty source at tree-level and stand-level was estimated.

Table IV-3 provides a summary of average uncertainty values by error source. The row in bold $(N D n C)$ is the reference error propagation explained in the previous section, in which all 
uncertainties were included. Rows $N D n C a, N D n C b$, and $N D n C a b$ are the individual error propagations by source of uncertainty $\left(a=u D B H, b=u T H\right.$ and $\left.a b=u \rho_{(D B H, T H)}\right)$. The row $N D n C c$ is the uncertainty of allometric model $(u A M)$.

\begin{tabular}{|c|c|c|c|c|c|c|c|c|c|c|c|c|}
\hline \multirow[b]{5}{*}{$\begin{array}{c}\text { uncertainty } \\
\text { source }\end{array}$} & \multicolumn{7}{|c|}{---- Non-sampling uncertainty (tree-level) ---- } & \multirow{2}{*}{\multicolumn{5}{|c|}{-- Total uncertainty (stand-level)-- }} \\
\hline & \multicolumn{5}{|c|}{---- Measurement uncertainty ---- } & \multirow{3}{*}{$\begin{array}{c}u^{2} \\
A M\end{array}$} & \multirow[b]{2}{*}{$u$} & & & & & \\
\hline & $u^{2}$ & $u^{2}$ & $u$ & $u$ & $u^{2}$ & & & $u^{2}$ & & $u^{2}$ & & \\
\hline & $D B H$ & $T H$ & $\rho_{D B H, T H}$ & Mes & Mes & & Tree & $N S$ & $S E^{2}$ & $W$ & $W$ & $R S E$ \\
\hline & $\%$ & $\%$ & $\%$ & $\begin{array}{c}\mathrm{kg} \\
\text { tree }^{-1}\end{array}$ & $\%$ & $\%$ & $\begin{array}{c}\mathrm{kg} \\
\text { tree }^{-1}\end{array}$ & $\%$ & $\%$ & $\begin{array}{l}M g \\
h a^{-1} \\
\end{array}$ & $\begin{array}{l}M g \\
h a^{-1} \\
\end{array}$ & $\%$ \\
\hline$N D n C$ & 29.0 & 44.0 & 27.0 & 11.41 & 1.7 & 98.3 & 88.16 & 1.26 & 98.74 & 13.87 & 176.07 & 7.88 \\
\hline $\mathrm{NDnCa}$ & 100 & 0 & 0 & 6.15 & 100 & 0 & 6.15 & 0.01 & 99.99 & 13.78 & 176.07 & 7.83 \\
\hline $\mathrm{NDnCb}$ & 0 & 100 & 0 & 7.57 & 100 & 0 & 7.57 & 0.02 & 99.98 & 13.78 & 176.07 & 7.83 \\
\hline$N D n C a b$ & 0 & 0 & 100 & 5.92 & 100 & 0 & 5.92 & 0.01 & 99.99 & 13.78 & 176.07 & 7.83 \\
\hline $\mathrm{NDnCc}$ & 0 & 0 & 0 & 0.00 & 0 & 100 & 87.42 & 1.21 & 98.79 & 13.86 & 176.07 & 7.87 \\
\hline
\end{tabular}

Table IV-3. The total uncertainty of the AGB calculation from non-sampling uncertainty estimation sources.

The total contribution of $u N S$ sources was $1.26 \%$, of which $0.01 \%$ came from $u D B H, 0.02 \%$ from $u T H, 0.01 \%$ from $u \rho_{(D B H, T H)}$, and $1.23 \%$ from $u A M$. The corresponding values in $\mathrm{Mg} \mathrm{ha}^{-1}$ were $u N S=1.56, u D B H=0.16, u T H=0.20, u \rho_{(D B H, T H)}=0.16$, and $u A M=1.53$. Applying the Equation 10 with the GUM Method, the total $u N S$ is

$$
1.56=\sqrt{(0.16)^{2}+(0.20)^{2}+(0.16)^{2}+(1.53)^{2}}
$$

The estimated mean uncertainties were compared by plot for each source of uncertainty through an ANOVA for repeated measurements (Tables VIII-11 and VIII-12, Appendix IV). In the analysis, significant differences between all sources were found, with a $\mathrm{p}$-value $=4.989 \mathrm{e}^{-07}$. In Table VIII-13 of Appendix IV, with a pairwise comparison, statistical difference was found ( $p$-value $<0.0205)$.

\section{IV.1.4.2 Monte-Carlo simulation Method (MCM)}

\section{IV.1.4.2.1 Total uncertainty analysis}

The scenarios applied in error propagation with $M C M$ used the uncertainty parameters (Tables VIII2 and VIII-3, Appendix II) of the PDF adjusted to the measurement errors in $D B H$ and $T H$. The normal distribution parameters were the same as in the GUM Method of error propagation. The scenarios were: normal distribution ( $M C N D n$ ), normal distribution adjusted by $D B H$ and $T H$ class $(M C N D n C)$, best adjusted distribution $(M C B D)$, and best adjusted distribution by $D B H$ and $T H$ class $(M C B D C)$.

Table IV-4 provides the error propagation using MCM. In this table, the results of the GUM Method were included. The percentage contribution of uncertainties due to non-sampling uncertainty $(u N S)$ ranged from 1.17 to $1.26 \%$ of the total uncertainty $(u W)$. The results show that more than $98.7 \%$ of the $u W$ was due to the sampling error $(S E)$. 
The highest $u N S$ contribution, $1.26 \%$, was with the GUM Method using normal distribution by classes $(N D n C)$. In contrast, the lowest contribution from $u N S$ was with the $M C M$ using normal distribution (MCNDn).

Because the $u W$ was nearly the same across all scenarios, the uncertainties at the plot level were compared through an Analysis of Variance for repeated measurements to determine if the applied scenarios were different.

\begin{tabular}{|c|c|c|c|c|c|c|}
\hline \multirow{2}{*}{$\begin{array}{l}\text { Uncertainty } \\
\text { propagation } \\
\text { method }\end{array}$} & \multirow{2}{*}{$\begin{array}{c}\text { parameters of } \\
\text { measurement } \\
\text { uncertainty }\end{array}$} & $\begin{array}{r}u^{2} \\
N S\end{array}$ & $S E$ & $u W$ & $\boldsymbol{W}$ & $R S E$ \\
\hline & & $\%$ & $\%$ & $M g h a^{-1}$ & $M g h a^{-1}$ & $\%$ \\
\hline \multirow{4}{*}{$M C M$} & $M C N D n$ & 1.17 & 98.83 & 13.86 & 176.11 & 7.87 \\
\hline & $M C N D n C$ & 1.19 & 99.81 & 13.86 & 176.14 & 7.87 \\
\hline & $M C B D$ & 1.23 & 98.77 & 13.86 & 176.18 & 7.87 \\
\hline & $M C B D C$ & 1.25 & 98.75 & 13.87 & 176.21 & 7.87 \\
\hline \multirow{3}{*}{$\begin{array}{l}\text { GUM } \\
\text { Method }\end{array}$} & $N D n$ & 1.23 & 98.77 & 13.87 & 176.07 & 7.88 \\
\hline & $N D n C$ & 1.26 & 98.74 & 13.87 & 176.07 & 7.88 \\
\hline & $R M S D$ & 1.25 & 98.75 & 13.87 & 176.07 & 7.88 \\
\hline
\end{tabular}

Table IV-4. Uncertainty of the AGB calculation with $M C M$ using four uncertainty estimation scenarios along with error propagations made with the GUM Method.

With this analysis, differences among the means of the evaluated scenarios were found ( $\mathrm{p}$ value $=0.0112$ ) and through the T-Test, the $\mathrm{p}$-value with Holm's corrections was calculated (Tables VIII-14 to 16, Appendix IV). Differences between the NDn and MCNDn scenarios were found. These scenarios used the same parameters of measurement uncertainty (p-value $=0.00021)$. Besides, differences between the $N D n C$ and $M C N D n C$ were observed, which also used the same uncertainty parameters in the measurement by class (p-value=0.00090). The $M C B D$ was also statistically different from the $N D n C$ and $R M S D$ (p-value<0.014), as well as $M C N D n$ (p-value<0.004).

\section{IV.1.4.2.2 Stand-level contribution by source of non-sampling uncertainty}

The contribution by source of $u N S$ for the scenario $M C B D C$ is in Table IV-5. The correlation between the $D B H$ and $T H$ variables was not included in the estimation, because it was less than 0.8 and was considered a negligible contribution. The sources of uncertainties are depicted in the following table as follows: $a=u D B H, b=u T H$ and $\mathrm{c}=u$ of allometric model $(u A M)$.

\begin{tabular}{|c|c|c|c|c|c|c|c|}
\hline \multirow{2}{*}{$\begin{array}{l}\text { uncertainty } \\
\text { source }\end{array}$} & $\begin{array}{r}u^{2} \\
N S \\
\end{array}$ & $S E$ & $u W$ & $\boldsymbol{W}$ & $\begin{array}{c}R S E \\
L L \\
\end{array}$ & $R S E$ & $\begin{array}{c}R S E \\
U L \\
\end{array}$ \\
\hline & $\%$ & $\%$ & $M g h a^{-1}$ & $M g h^{-1}$ & $\%$ & $\%$ & $\%$ \\
\hline$M C B D C$ & 1.25 & 98.75 & 13.87 & 176.21 & 7.41 & 7.87 & 8.32 \\
\hline$M C B D C a$ & 0.03 & 99.97 & 13.80 & 176.23 & 7.76 & 7.83 & 7.90 \\
\hline$M C B D C b$ & 0.03 & 99.97 & 13.78 & 176.05 & 7.76 & 7.83 & 7.89 \\
\hline$M C B D C c$ & 1.20 & 98.80 & 13.86 & 176.09 & 7.42 & 7.87 & 8.30 \\
\hline
\end{tabular}

Table IV-5. Uncertainty of the AGB calculation with $M C M$ for non-sampling uncertainty sources and their contribution to the total uncertainty. 
The contributions of $u D B H\left(0.233 \mathrm{Mg} \mathrm{ha}^{-1}\right)$ and $u T H\left(0.230 \mathrm{Mg} \mathrm{ha}^{-1}\right)$ were $0.03 \%$ each. The $u A M$ had the largest contribution for the $u N S$ with $1.20 \%\left(1.52 \mathrm{Mg} \mathrm{ha}^{-1}\right)$. Using the contribution per ha of every source in Equation 15, the result of $M C M$ per source of uncertainty was shown to be an approximation for the total $N S$ uncertainty.

$$
1.55 \approx 1.56=\sqrt{(0.233)^{2}+(0.230)^{2}+(1.52)^{2}}
$$

The uncertainty due to sampling contributed $98.75 \%$ to the total uncertainty. In the columns following the estimated AGB $(W)$ are the values of the relative standard error $(R S E)$ estimate. With the $M C M$, not only the average value of the estimate was reported, but also a $95 \%$ confidence interval. Therefore, the RSE LL column was the lower limit and the RSE $U L$ column was the upper limit of the relative uncertainty.

Comparing the mean uncertainties (Tables VIII-17 to 19, Appendix IV) in the AGB estimation by plot, significant differences were found among the contributions by the source of uncertainty and the $u W$ per plot (p-value $<2.722 \mathrm{e}^{-06}$ ). The mean uncertainties in AGB estimation by $D B H$ and $T H$ were not significantly different ( $\mathrm{p}$-value $=0.708)$. However, the other paired comparisons were significantly distinct ( $\mathrm{p}$-value $\leq 0.034)$. 
IV.2 Empirical study 2: Comparison of aboveground biomass estimates from two types of dasometric measuring equipment for the variables $D B H$ and $T H$, a case study developed in beech forest near Göttingen, Germany

\section{IV.2.1 General information}

In total, 1103 trees of 16 genera were registered in the 47 plots of the study area. The genus Fagus spp dominated, with $47 \%$ of the measured trees, which, together with the genera Acer spp, Fraxinus spp, and Prunus spp, accounted for $87 \%$ of the total number of registered trees.

Applying the simple random sampling estimators, the estimates per-hectare of the number of trees, basal area, and AGB were calculated for the re-measurement plots and the stand area. Table IV-6 shows the results range and Table IV-7 shows the mean value of measured and estimated variables per device for both the stand area (47 plots) and the re-measurement plots (11 plots).

\begin{tabular}{|c|c|c|c|c|}
\hline \multirow{2}{*}{\multicolumn{2}{|c|}{ Variable }} & \multicolumn{2}{|c|}{ Re-measurement (11 plots) } & \multirow{2}{*}{$\begin{array}{c}\text { Stand area } \\
\text { (47 plots) } \\
\text { Tape+Vertex }\end{array}$} \\
\hline & & Caliper+Blume-Leiss & Tape+Vertex & \\
\hline \multirow[b]{2}{*}{ Measured } & $D B H(\mathrm{~cm})$ & $6.9-62.4$ & $7.0-63.6$ & $7.8-89.8$ \\
\hline & $T H(\mathrm{~m})$ & $5.2-36.6$ & $5.0-34.7$ & $7.3-36.3$ \\
\hline \multirow{3}{*}{ Estimated } & No. of trees $\left(h^{-1}\right)$ & \multicolumn{2}{|c|}{$320-660$} & $160-1360$ \\
\hline & Basal area $\left(\mathrm{m}^{2} \mathrm{ha}^{-1}\right)$ & $21.1-39.1$ & $21.4-39.8$ & $15.9-48.2$ \\
\hline & $\operatorname{AGB}\left(\mathrm{Mg} \mathrm{ha}^{-1}\right)$ & $159.3-314.4$ & $154.8-325.1$ & $88.2-424.5$ \\
\hline
\end{tabular}

Table IV-6. Range of values for measurements and estimated variables in the study area.

\begin{tabular}{|c|c|c|c|c|}
\hline \multirow{2}{*}{\multicolumn{2}{|c|}{ Variable }} & \multicolumn{2}{|c|}{ Re-measurement (11 plots) } & \multirow{2}{*}{$\begin{array}{c}\text { Stand area } \\
(47 \text { plots }) \\
\text { Tape+Vertex }\end{array}$} \\
\hline & & Caliper + Blume-Leiss & Tape+Vertex & \\
\hline \multirow{2}{*}{ Measured } & $D B H(\mathrm{~cm})$ & 23.9 & 24.2 & 25.3 \\
\hline & $T H(\mathrm{~m})$ & 19.9 & 19.7 & 20.0 \\
\hline \multirow{3}{*}{ Estimated } & No. of trees $\left(\mathrm{ha}^{-1}\right)$ & \multicolumn{2}{|c|}{455} & 469 \\
\hline & Basal area $\left(\mathrm{m}^{2} \mathrm{ha}^{-1}\right)$ & 28.8 & 29.4 & 32.1 \\
\hline & AGB $\left(\mathrm{Mg} \mathrm{ha}^{-1}\right)$ & 236.3 & 238.6 & 257.6 \\
\hline
\end{tabular}

Table IV-7. Statistics of measurements and estimated variables in the study area.

The basal area and AGB per class estimates are plotted in Figure IV-5. The tree frequency bars were the same for basal area and AGB estimates, and tree frequency varied depending on DBH or TH classes. In the four graphs of this figure, the pattern observed in the estimation of basal area and AGB was similar by class and by measuring device. As shown in the upper graphs, the number of trees per $D B H$ class was similar between the stand and re-measurement plots. The smaller classes $(7-20 \mathrm{~cm}]$ had the lowest contribution to basal area and AGB, despite having registered more than $50 \%$ of the sampled trees. In contrast, with less than $22 \%$ of the trees sampled, large trees (40-63 $\mathrm{cm}$ ) contributed to more than $60 \%$ of the basal area and more than $65 \%$ of AGB.

The estimate of AGB and basal area follow similar patterns by $T H$ class. However, the proportion of the $T H$ classes had greater variation. For example, the $T H$ measurements between the remeasurement plots showed a smaller number of trees in the intermediate class (15-25 m). The 
largest trees in $\mathrm{TH}$ (from $25 \mathrm{~m}$ ) had the largest contribution to the estimated variables, more than $71 \%$ of the total basal area and more than $79 \%$ of the AGB.

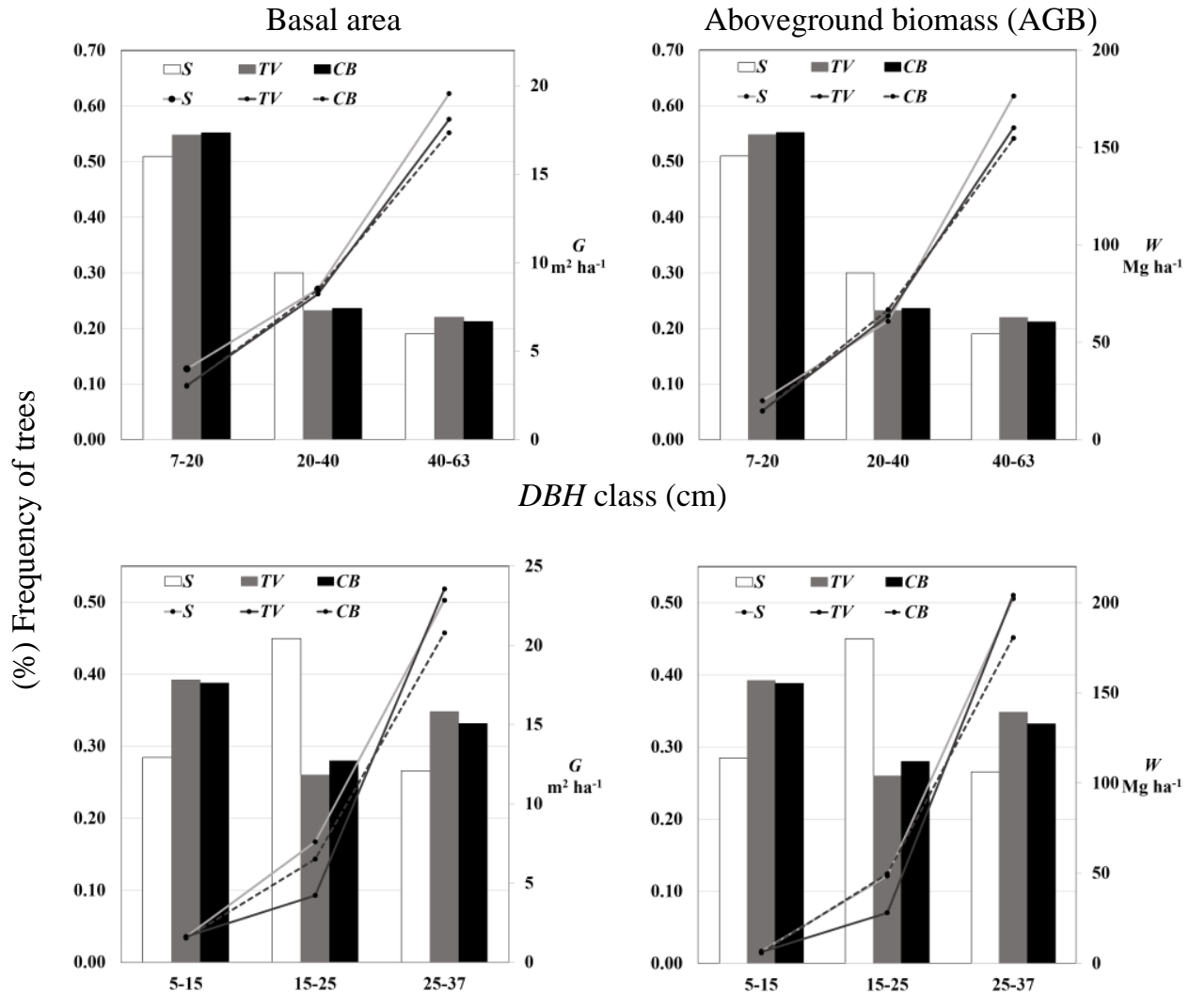

$T H$ class (m)

$S=$ Stand-level, $T V=$ re-measurement with Tape+Vertex and $C B=$ re-measurement with Caliper+Blume-Leiss.

Figure IV-5. Basal area and AGB estimation. Above, estimate by $D B H$ class every $20 \mathrm{~cm}$; below, estimate by $T H$ class every $10 \mathrm{~m}$. The bars represent the frequency of threes per size class.

\section{IV.2.1.1 DBH and $\mathrm{TH}$ relation}

Figure IV-6 shows the relationship between $D B H$ and $T H$. The figure depicts the mean values of the five re-measurements made with Caliper for $D B H$ and with the Blume-Leiss for $T H(C B)$. In the same way, the mean values measured by the Tape and Vertex (TV) were plotted in the same figure. Comparing the two datasets values less than $25 \mathrm{~cm} \mathrm{DBH}$ and less than $24 \mathrm{~m} \mathrm{TH}$, had a similar dispersion. Higher values of both variables showed greater dispersion.

Pearson's correlation coefficients $(\rho)$ are also included in the graph. In both cases, the coefficient was positive and greater than 0.92 , showing the systematic variation between the two variables, as well as the direction of the relationship. This coefficient was used in the error propagation as stated the Equation $06 b$.

\section{IV.2.1.2 Data sets comparison}

\section{IV.2.1.2.1 Diameter at breast height (DBH)}

The differences between variables and devices were analyzed through boxplots and frequency histograms in Figure IV-7. The left side shows the comparison of $D B H$ measured by Caliper $(C)$ 
and by Tape (T). The results in the boxplots for the two devices were similar, with no extreme values observed. The median, from the boxplots, for Caliper was $18.21 \mathrm{~cm}$ and for Tape was 18.32 $\mathrm{cm}$.

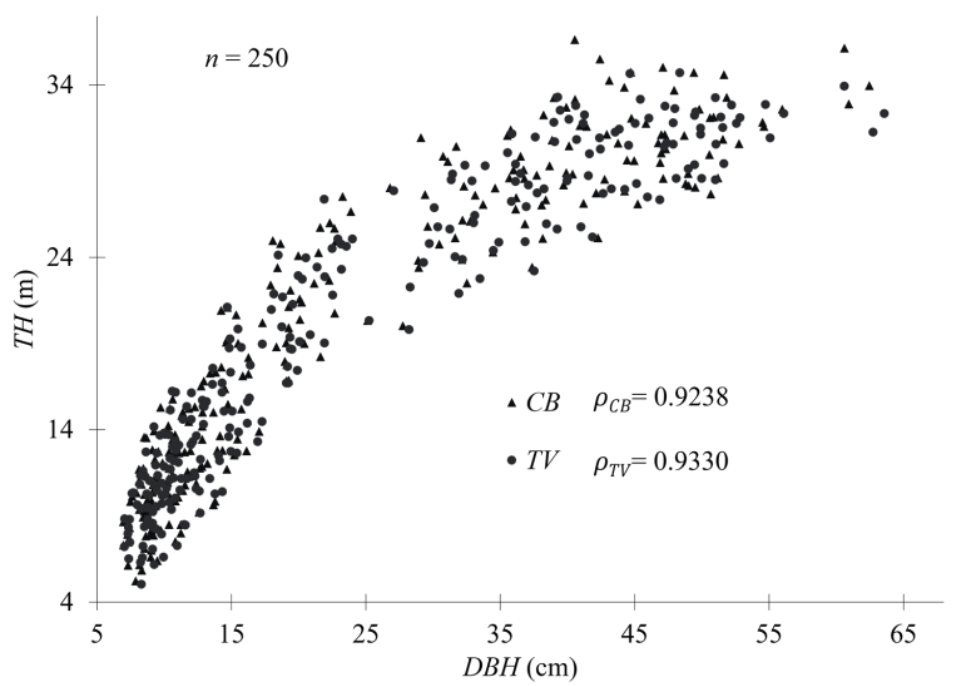

Figure IV-6. Relationship between $D B H$ and $T H$ for mean values of the 250 trees in remeasurement plots made with Caliper and Blume-Leiss $(C B)$ and with Tape and Vertex (TV).

The histogram in Figure IV-7 (top left) displays the measurement frequencies by class for each device and the areas of overlap $(0.98 \%)$ indicate that the measurements made by both devices had the same frequency. Two frequency bars (0-10 and 20-30) had a higher frequency for Caliper, and one bar (40-50) recorded more trees for Tape measurements. In the lower part, the scatter plot denotes a high relationship between Caliper and Tape measurements per tree, with a Pearson correlation coefficient $\rho_{\text {(caliper, } \text {, tape })}=0.99$.

The Wilcoxon Signed-Rank Test compared the data pairs and resulted in a p_value ${ }_{\mathrm{Wrt}}<2.2 \mathrm{e}^{-16}$, indicating non-identical populations of the two datasets $(C$ and $T$ ) of best estimates . Before the median analysis, Bartlett's Test was applied $\left(p\right.$-value $\left._{\mathrm{Bt}}=0.9263\right)$ and verified homogeneous variances.

\section{IV.2.1.2.2 Total height (TH)}

The histogram in Figure IV-7 (top right) shows that only in the class 20-25 both devices had the same frequency of measured trees. For the other six classes, each device had three classes in which it was the most frequent. In total, there was $95.6 \%$ overlap between the two devices. As shown in the boxplot, the Vertex provided fewer extreme values. This trend was also registered in the lower median value of $18.87 \mathrm{~m}$ for the Vertex and $19.05 \mathrm{~m}$ for the Blume-Leiss.

The datasets were not normally distributed, so the Wilcoxon Signed-Rank Test for paired data was used to compare them. A p-value $\mathrm{wr}_{\mathrm{rt}}=0.0539$ was calculated, indicating that the difference between the medians of the two datasets included zero and that there are no significant differences between the datasets. 

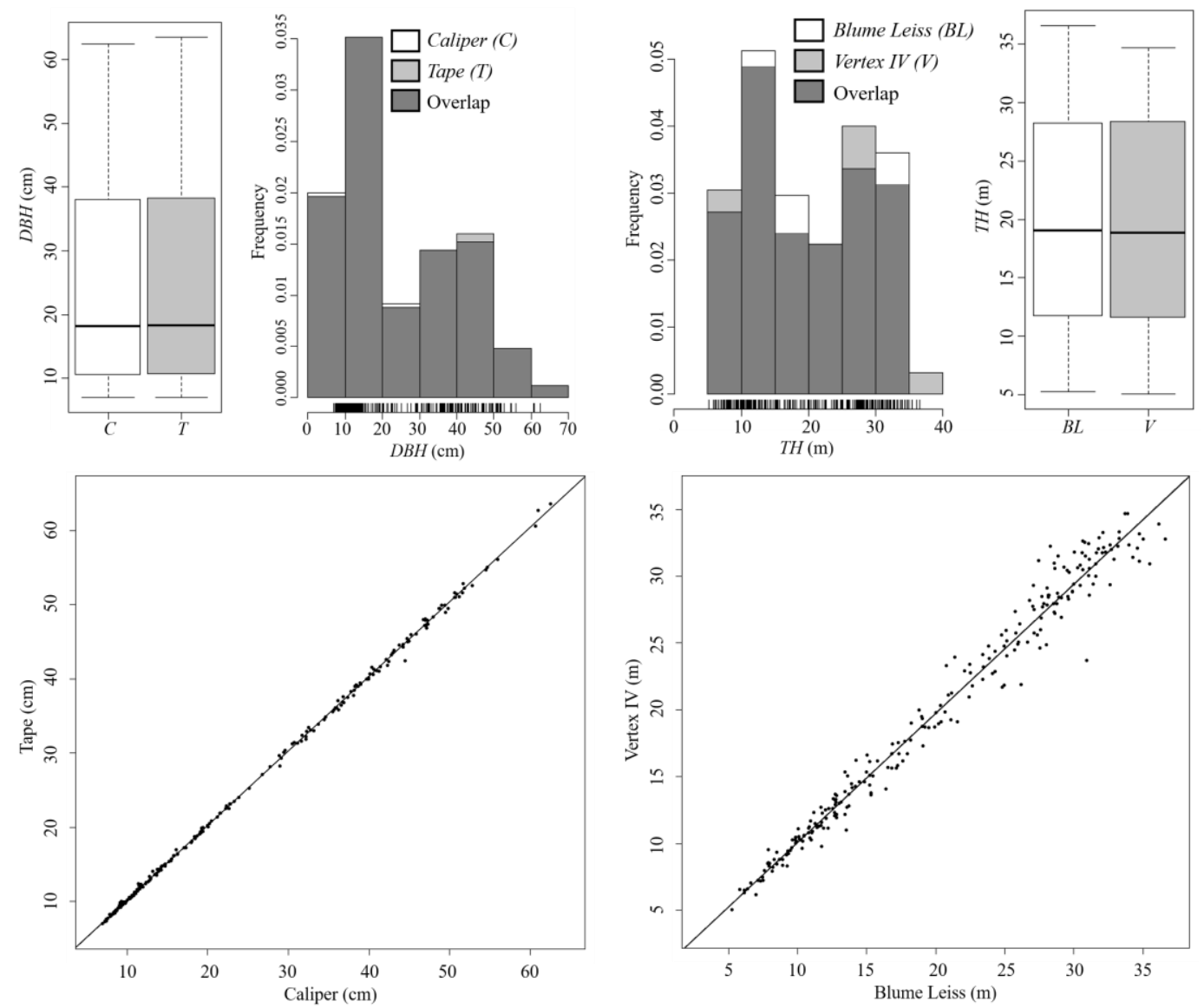

$D B H$

TH

Figure IV-7. Left-top: $D B H$ boxplot and overlapped histograms of Caliper $(C)$ and Tape $(T)$ measurements. Right-top, graphs of TH made with Blume-Leiss $(B L)$ and Vertex (V). Bottom: comparison of measurements made with two devices.

\section{IV.2.2 Measurement error}

\section{IV.2.2.1 Individual tree uncertainty}

One of the methods used to propagate the uncertainties was the GUM Method using the Law of Error Propagation. This method assumes a normal distribution of the error, so the variable "measurement error" for each tree with the SRS standard deviation estimator was estimated.

\section{IV.2.2.1.1 Diameter at breast height (DBH)}

The Caliper measurement errors ranged from 0 to $1.01 \mathrm{~cm}$, while the Tape measurement error ranged from 0.01 to $0.63 \mathrm{~cm}$. Considering the maximum value of measurement errors with Tape $(0.63 \mathrm{~cm}), 96 \%$ of the error with Caliper fell within the range of errors of Tape. The mean value of the error for each device was $0.19 \mathrm{~cm}$ and $0.16 \mathrm{~cm}$, for Caliper and Tape.

\section{IV.2.2.1.2 Total height (TH)}

The measurement errors estimated for $T H$ had limit values from 0.03 to $2.22 \mathrm{~m}$ for Blume-Leiss $(B L)$ and 0.01 to $1.85 \mathrm{~m}$ for Vertex. The mean value of measurement error was 0.88 for $B L$ and 0.58 
m for Vertex. $97.2 \%$ of the errors measured with $B L$ were less than or equivalent to the maximum measurement error made by Vertex $(1.85 \mathrm{~m})$.

\section{IV.2.2.2 Measurement uncertainty parameters for the study area.}

\section{IV.2.2.2.1 GUM Method}

The parameters used to propagate the errors of AGB estimates are shown in Table IV-8. In this table, were included two types of results: those which assumed homoscedasticity in the measurement errors, and those which assumed heteroscedasticity in the measurement errors, as described in Section III.2.4.1.2.3. The applied parameter was the standard deviation $(\hat{\sigma})$, expressed in centimeters for $D B H$ and meters for $T H$. Following the terminology established in Section III.2.6.3, in the first scenario, called "Mean", was used the mean value of all measurement errors. The second scenario was "NDn", with the dispersion parameter estimated by fitting the errors to a normal distribution (Tables VIII-4 to VIII-7, Appendix III).

In this table, the parameters of students with (Exp) and without (NExp) previous experience in forest mensuration were included, from Section III.2.2.2. At the end of the table are the models fitted by linear regression (scenario 4 ) to estimate $\hat{\sigma}$, which follows the heteroscedasticity of the errors (Figure VIII-1, Appendix III). The scenario 3 "NDnC" (not included in the table due to compatibility of size), fitting the errors to a normal distribution by class, the dispersion parameters are in Appendix III (Tables VIII-4 to VIII-7).

\begin{tabular}{|c|c|c|c|c|c|c|}
\hline \multirow{4}{*}{ Variable } & \multirow{4}{*}{ Device } & \multicolumn{5}{|c|}{ Scenarios of measurement error estimation $(\hat{\sigma})$} \\
\hline & & \multicolumn{4}{|c|}{ Homoscedastic errors } & \multirow{3}{*}{$\begin{array}{l}\text { Heteroscedastic errors } \\
\text { Linear Regression }^{1}\end{array}$} \\
\hline & & \multirow[t]{2}{*}{ Mean } & \multirow{2}{*}{$N D n$} & \multicolumn{2}{|c|}{ Students } & \\
\hline & & & & $\operatorname{Exp}$ & Nexp & \\
\hline$D B H$ & Caliper & 0.193 & 0.212 & - & - & $\left(\left((-2.012+0.018 * D B H)^{*} \lambda\right)+1\right)^{1 / \lambda}$ \\
\hline$(\mathrm{cm})$ & Tape & 0.162 & 0.209 & 1.178 & 1.183 & $\left(\left((-1.993+0.018 * D B H)^{*} \lambda\right)+1\right)^{1 / \lambda}$ \\
\hline$T H$ & Blume-Leiss & 0.882 & 1.229 & - & - & $\left(\left((-0.667+0.024 * T H)^{*} \lambda\right)+1\right)^{1 / \lambda}$ \\
\hline$(\mathrm{m})$ & Vertex & 0.576 & 0.646 & 4.01 & 4.06 & $\left(\left((-1.488+0.037 * T H)^{*} \lambda\right)+1\right)^{1 / \lambda}$ \\
\hline
\end{tabular}

Table IV-8. Uncertainty parameters for scenarios to estimate measurement error of $D B H$ and $T H$.

\section{IV.2.2.2.2 Monte-Carlo simulation Method}

The $M C M$ did not require the assumption of normality in error distribution. Therefore, the distributions of measurement errors were fit to the PDF that best described those errors (Figure IV$8 \mathrm{a}$ and $\mathrm{b}$ ). To be consistent with homoscedasticity in the measured errors, the PDFs by class were adjusted (Figure IV-8c to h), as established in Section III.2.4.1.2.2.

Shown in Figure IV-8 are the PDFs which best fit to the measurement errors by diameter class (black line), along with the normal distribution (gray line) for reference. The number of trees per 
class are also displayed next to each graph. The adjustment parameters, as well as the goodness-offit per PDF, are recorded in Tables VIII-4 and VIII-5 of Appendix III.

The logistic and cosine distributions were closely aligned to normal distribution. However, in the PDFs for Caliper [20-40) and Tape [40-63], the trapezoidal distribution had a better fit.

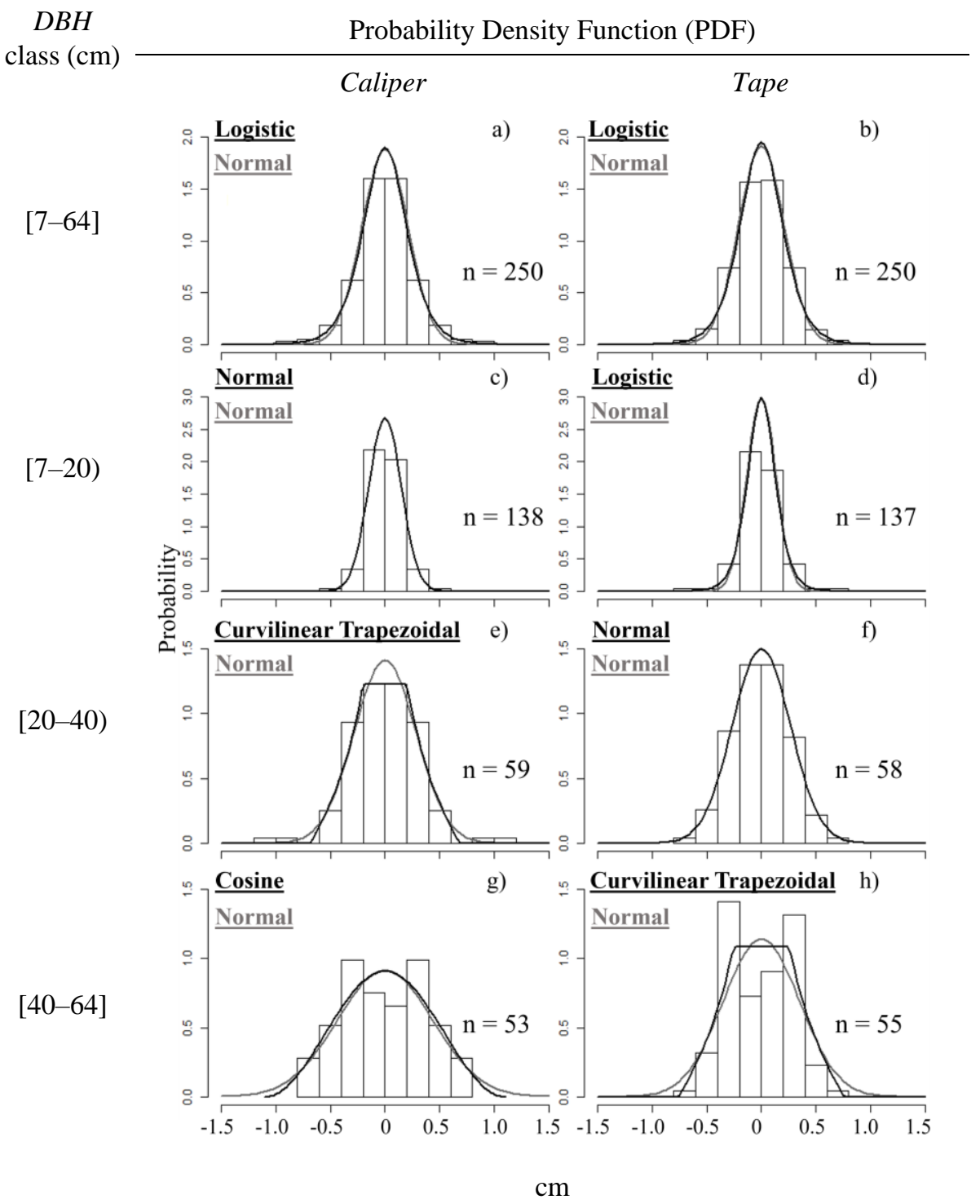

Figure IV-8. Probability Density Function (PDF) fitted with $D B H$ measurement error according to DBH class, for Caliper and Tape measurement. a) and b) are PDF fitted for the whole dataset of Caliper and Tape, respectively.

Distributions adjusted for $T H$ measurement errors are found in Figure IV-9. The parameters for the PDF's adjusted are in Tables VIII-6 and VIII-7 of Appendix III.

The $T H$ measurement errors showed larger differences between the best-fit distribution (black line) and the normal distribution (gray line) than measurement errors in $D B H$. The errors measured by Blume-Leiss were greater than those measured by Vertex. This observation was verified graphically 
by noting that the Blume-Leiss had more frequent large errors and that the parameters of the normal distributions were smaller for the errors measured by the Vertex.

TH

class (m)

$[5-15)$

$[15-25)$

[25-37]
Probability Density Function (PDF)

Blume-Leiss Vertex

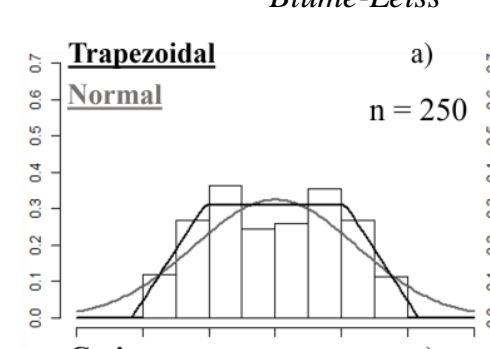

c)

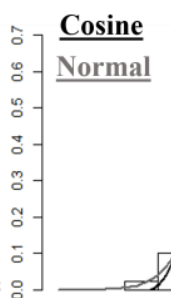

Von Mises

d)
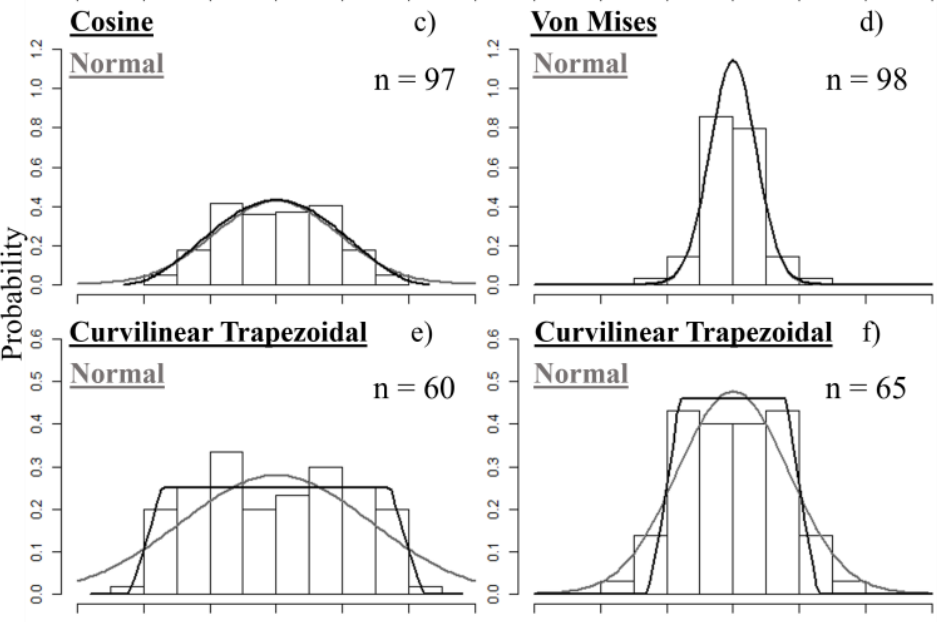

Normal

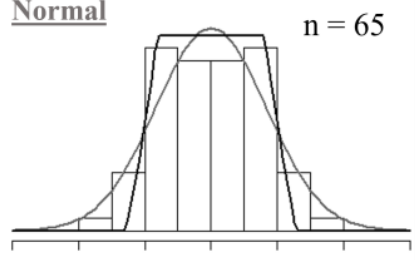

g) :

Normal b)

$\mathrm{n}=250$
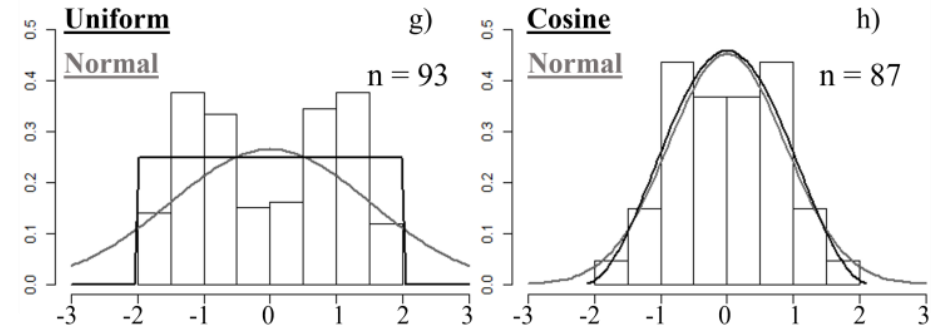

$\mathrm{m}$

Figure IV-9. Probability Density Function fitted with $T H$ measurement error according to $T H$ class, for Blume-Leiss and Vertex measurements. a) and b) are PDF fitted for the whole dataset of BlumeLeiss and Vertex $I V$, respectively.

\section{IV.2.3 Error propagation}

\section{IV.2.3.1 GUM Method of error propagation}

\section{IV.2.3.1.1 Measurement error propagation by tree}

In this Section, the AGB and the uncertainty of AGB estimation by four datasets were compared. The datasets identification is with the first letter of the measurement devices. In this way, the first two datasets were CB (Caliper+Blume Leiss) and TV (Tape+Vertex), and the AGB predictions were 
made with the two-variables model. The other two datasets were $C$ (Caliper) and $T$ (Tape) and the AGB was predicted with the one-variable model.

The error propagation required the error parameters (Section IV.2.2.2.1) from the two non-sampling sources and the correlation of the two variables measured in the field. The Pearson correlation coefficient $(\rho)$ was calculated by tree with the matrix made up of the five $D B H$ re-measurements and the five $T H$ re-measurements. The error propagation is in Table IV-9.

In the upper part of the table are the uncertainty values in the units of the measurement. In the lower part, the percentage values show the proportional contribution from every source to the propagated error. The non-sampling uncertainty information was the average data of the 250 measured trees.

Measurement uncertainty of an average tree ( $u$ Wtree in column 4$)$ was lower for $T V$ than for $C B$. TH had the largest contribution to measurement error, with values of $62 \%$ for Blume-Leiss and $56.3 \%$ for Vertex. The contribution of $\mathrm{DBH}$ to $u$ Wtree was between 22.7-30.2\%, and the correlation contributed to less than $15.3 \%$. In scenarios using only $D B H$ in the AGB model ( $C$ and $T), D B H$ uncertainty was larger than $D B H$ uncertainty in scenarios using $D B H$ and $T H$. However, the total AGB estimate at tree-level was less than the other estimates.

\begin{tabular}{|c|c|c|c|c|c|c|c|c|c|c|c|c|c|}
\hline \multirow{8}{*}{ 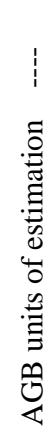 } & & \multicolumn{7}{|c|}{--------- Non-sampling uncertainty $(u)$--------- } & \multicolumn{5}{|c|}{$\begin{array}{l}\text { Uncertainty }(u) \text { in the } 11 \text { plots of } \\
\text { re-measurement }\end{array}$} \\
\hline & & 1 & 2 & 3 & \multicolumn{2}{|c|}{4} & 5 & 6 & 7 & 8 & 9 & 10 & 11 \\
\hline & & $u$ & $u$ & $u$ & \multicolumn{2}{|l|}{$\boldsymbol{u}$} & $u$ & $u$ & $u$ & & $u$ & & $u$ \\
\hline & & $D B H$ & $T H$ & $\rho_{D B H, T H^{*}}$ & \multicolumn{2}{|c|}{ Mes } & $A M$ & Tree & $N S$ & $S E$ & $W$ & $\boldsymbol{W}$ & $W_{E X P}$ \\
\hline & Uncertainty & $\mathrm{kg}$ & $\mathrm{kg}$ & $\mathrm{kg}$ & \multirow{2}{*}{\multicolumn{2}{|c|}{$\begin{array}{c}\text { kg } \\
\text { tree }^{-1}\end{array}$}} & $\mathrm{~kg}$ & kg & $\mathrm{Mg}$ & $\mathrm{Mg}$ & $\mathrm{Mg}$ & Mg & $\mathrm{Mg}$ \\
\hline & scenario & tree $^{-1}$ & $\operatorname{tree}^{-1}$ & $\operatorname{tree}^{-1}$ & & & tree $^{-1}$ & tree $^{-1}$ & $\mathrm{ha}^{-1}$ & $\mathrm{ha}^{-1}$ & $\mathrm{ha}^{-1}$ & ha $^{-1}$ & $\mathrm{ha}^{-1}$ \\
\hline & $C B$ & 8.70 & 14.38 & 7.16 & \multicolumn{2}{|r|}{18.26} & 1.30 & 18.31 & 0.87 & 13.71 & 13.74 & 236.28 & \pm 26.93 \\
\hline & $T V$ & 6.68 & 9.12 & 4.46 & \multicolumn{2}{|r|}{12.16} & 1.30 & 12.23 & 0.55 & 14.42 & 14.43 & 238.64 & \pm 28.28 \\
\hline 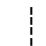 & $C$ & 9.01 & - & - & \multicolumn{2}{|r|}{9.01} & 1.35 & 9.11 & 0.50 & 14.80 & 14.81 & 223.40 & \pm 29.03 \\
\hline & $T$ & 6.99 & - & - & \multicolumn{2}{|r|}{6.99} & 1.35 & 7.12 & 0.32 & 15.03 & 15.04 & 228.64 & \pm 29.47 \\
\hline \multirow{9}{*}{$\begin{array}{l}50 \\
0 \\
0 \\
\mathbb{\Xi} \\
0 \\
0 \\
0 \\
0 \\
0\end{array}$} & & \multicolumn{7}{|c|}{ - } & \multirow{2}{*}{\multicolumn{5}{|c|}{$\begin{array}{l}\text { Uncertainty }(u) \text { in the } 11 \text { plots of } \\
\text { re-measurement }\end{array}$}} \\
\hline & & \multicolumn{7}{|c|}{--------- Measurement ---------- } & & & & & \\
\hline & & $u^{2}$ & $u^{2}$ & $u$ & $u$ & $u^{2}$ & $u^{2}$ & $u$ & $u^{2}$ & & $u$ & & \\
\hline & & $D B H$ & $T H$ & $\rho_{D B H, T H}$ & Mes & Mes & $A M$ & Tree & $N S$ & $S E^{2}$ & $W$ & $\boldsymbol{W}$ & $R S E$ \\
\hline & $\begin{array}{c}\text { Uncertainty } \\
\text { scenario }\end{array}$ & $\%$ & $\%$ & $\%$ & $\begin{array}{c}\text { kg } \\
\text { tree }^{-1}\end{array}$ & $\%$ & $\%$ & $\begin{array}{c}\text { kg } \\
\text { tree }^{-1}\end{array}$ & $\%$ & $\%$ & $\begin{array}{l}\mathrm{Mg} \\
\mathrm{ha}^{-1}\end{array}$ & $\begin{array}{l}\text { Mg } \\
\text { ha }^{-1}\end{array}$ & $\%$ \\
\hline & $C B$ & 22.7 & 62.0 & 15.3 & 18.26 & 99.5 & 0.5 & 18.31 & 0.40 & 99.60 & 13.74 & 236.28 & 5.82 \\
\hline & $T V$ & 30.2 & 56.3 & 13.5 & 12.16 & 98.9 & 1.1 & 12.23 & 0.15 & 99.85 & 14.43 & 238.64 & 6.05 \\
\hline & $C$ & 100 & - & - & 9.01 & 97.8 & 2.2 & 9.11 & 0.11 & 99.89 & 14.81 & 223.40 & 6.63 \\
\hline & $T$ & 100 & - & - & 6.99 & 96.4 & 3.6 & 7.12 & 0.05 & 99.95 & 15.04 & 228.64 & 6.58 \\
\hline
\end{tabular}

Table IV-9. Uncertainty of the AGB calculation using four scenarios to estimate the measurement uncertainty Caliper+Blume-Leiss (CB), Tape+Vertex (TV), Caliper (C) and Tape (T).

$u$ Wtree combined with the allometric model uncertainty $(u A M)$ resulted in the non-sampling uncertainty $(u N S)$. The Allometric models contributed $\leq 3.6 \%$ to the $u N S$. Therefore, the largest contribution to $u N S$ was $u W$ tree, with more than $96.4 \%$ of the contribution. 
The estimates per tree were aggregated at the plot-level and expanded to values per hectare (ha). This information is summarized in column 7 from Table IV-9. uNS combined with $S E$ resulted in the total uncertainty $(u W)$. The estimate of $u N S$ was $\leq 0.87 \mathrm{Mg} \mathrm{ha}^{-1}(0.4 \%)$ and from $S E$ it was $\geq 13.71 \mathrm{Mg} \mathrm{ha}^{-1}(99.6 \%)$.

The estimated mean of AGB (column 10) was higher in calculations using two variables in the estimate of AGB ( $C B$ and $T V$ ), than with one variable to estimate AGB ( $C$ and $T$ ).

The four AGB estimates were compared using the estimates per plot. Appendix V shows the result of the Analysis of Variance for repeated measurements and the paired T-Test applied to the four AGB estimates (Tables VIII-20 to VIII-22). The ANOVA Test indicated no significant difference for the $C B$ and $T V$ estimates (p-value $=0.2916$ ). However, the other pairs' comparisons showed a significant difference ( $\mathrm{p}$-value $\leq 0.0256)$.

The total error of the four AGB estimates was also compared (Tables VIII-23 to VIII-25, Appendix V). Significant differences were found in the analysis for total estimated errors per plot with scenarios $C B$ and $C$ (p-value $=0.00034)$ and for total estimated errors in scenarios $T V$ and $T$ (pvalue $=0.00051)$.

\section{IV.2.3.1.2 Measurement error propagation by tree (modeled)}

In this section, the comparison of the measured uncertainty with the scenarios to estimate measurement errors is shown (Section III.2.4.1.1). The GUM Method of error propagation was used to make the comparison, applying the scenarios to estimate the measurement error to the datasets Caliper+Blume-Leiss $(C B)$ and Tape+Vertex (TV).

The uncertainty parameters in Section IV.2.2.2.1, together with the correlations of the measured dataset $\left(\rho_{C B}=0.9238, \rho_{T V}=0.9330\right)$ were used to propagate the error in AGB estimation. The results of the propagations are in Table IV-10. This table includes the reference information estimated in the previous Section for the $C B$ and $T V$ datasets (bold rows).

The applied scenarios, in Table III-6 of Section III.2.6.3, were previously described. The scenario used the dataset $(C B$ or $T V$ ), together with the method to estimate the measurement errors: average uncertainty value (mean), normal distribution $(N D n)$, normal distribution per class $(N D n C)$, students with prior experience (Exp) and students without prior experience (Nexp).

The table shows the percentages that each source contributed to the estimated uncertainty at different levels. The estimated levels were as follows: measurement uncertainty (uMes in column 4), non-sampling uncertainty at tree-level (uTree in column 7), and total uncertainty ( $u W$ in column 10). Column 12 shows the relative standard error $(R S E)$, which gives an estimate of $u W$ in relation to the total AGB estimate $(W)$.

From the error propagation with the Caliper+Blume-Leiss $(C B)$ measurements, it was observed that $u T H$ had the highest contribution to the $u M e s$ (column 4). This error contributed, on average, to at least $41.1 \%$ of the error, and was greater than the contribution of $u D B H$ in all the scenarios (less than 28\%). The uMes of the CBNDn, CBNDnC, and CBmod scenarios overestimated the reference estimation $\left(C B=18.26 \mathrm{~kg}\right.$ tree $\left.^{-1}\right)$. The use of the $C B m e a n$ scenario led to an underestimation of the $u M e s$ and the $R S E$ of the reference value. 
The contribution of the AGB prediction model $(u A M)$ to $u N S$ was $\leq 0.7 \%$. Therefore, the largest contribution to $u N S$ was the $u M e s$, with over $99.3 \%$ of the estimate in column 7. Due to the low contribution of $u A M$, the $u$ Tree had a slight increase compared to the estimates in $u M e s$ (column 4).

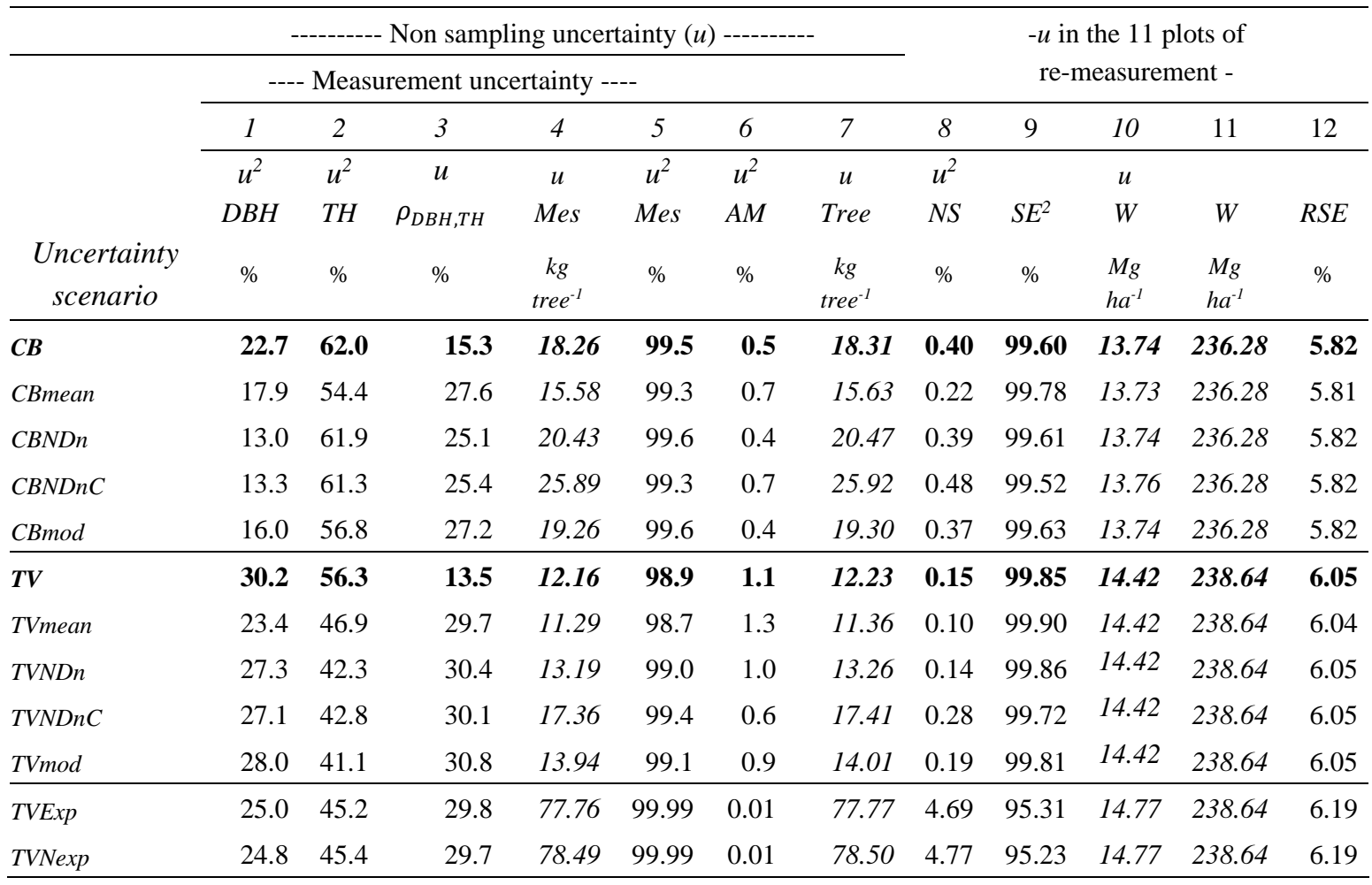

Table IV-10. Sources of uncertainty contribution in the AGB calculation for different scenarios of measurement error estimation.

Two scenarios had the same value of $u W$ as the reference value. These scenarios, CBNDn and $C B m o d$, had the $u N S$ contribution closest to the reference value $(0.40 \%)$. The estimation of the relative standard error $(R S E)$ was alike for all scenarios because the main source of error was always $S E$, accounting for more than $99.46 \%$ of the contribution.

To compare the scenarios using the $C B$ dataset, an ANOVA for repeated measurements was performed with the estimated mean uncertainty per plot. The results of the ANOVA are in Appendix V (Tables VIII-26 to VIII-28). The findings indicate that the CBmod (p-value=0.10039) and $C B N D n$ (p-value $=0.97838$ ) scenarios did not differ significantly from the reference estimate.

The propagations using the $T V$ dataset are at the bottom of Table IV-10. The TVmean, when compared to reference value $T V$, was the only scenario that underestimated $u$ Wtree (column 4). At this estimation level, the main contribution for all scenarios was $u T H(\geq 41.1 \%)$, followed by the correlation $(\leq 30.8 \%)$, and with the least contribution from the $u D B H(\leq 28.0 \%)$.

For the uTree (column 7), the contribution of allometric models $(u A M)$ was less than or equal to $1.3 \%$, while $u M e s$ contributed more than $98.7 \%$. Converting the $u N S$ to values per ha and combining it with the sampling error $(S E)$ resulted in a total uncertainty of AGB ( $u W$ in column 10). The greatest contribution of this uncertainty was the $S E$ at more than $99.72 \%$. The $R S E$ value 
followed the initial trend, where the TVmean scenario underestimated the result of the reference value $T V$.

The analysis, ANOVA for repeated measurements, of the mean estimated uncertainties per plot (Tables VIII-29 to VIII-31, Appendix V), determined that the TVmod (p-value=0.521) and TVNDn ( $\mathrm{p}$-value $=0.71675$ ) estimates did not differ significantly from the mean uncertainties estimated by the measured uncertainty $T V$.

The errors propagated with the parameters of measurement errors made by students, TVExp and TVNexp, showed similar values for the three levels of uncertainty. Comparing the errors propagated in TVExp and TVNexp with the measured uncertainty for measurements with Tape+Vertex (TV), the uMes and the uTree were six times larger. This deviation resulted in a larger contribution to the final measurement error of almost $5 \%$ of the $u W$. This contribution increased $R S E$ from $6.05 \%$ (reference scenario) to $6.19 \%$ for TVExp and TVNexp.

The mean uncertainty in AGB estimation per plot calculated with the students' measurement errors (TVExp and TVNexp) were compared, with the measured uncertainty TV by using an ANOVA for repeated measurements (Tables VIII-32 to 34, Appendix V). The difference between the mean uncertainty values was significant $\left(\mathrm{p}\right.$-value $\left.=2.321 \mathrm{e}^{-12}\right)$, and, from applying a paired T-Test $($ Holm correction), it was found that significant differences exist for the mean uncertainties in these three scenarios ( $\mathrm{p}$-value $\leq 9.2 \mathrm{e}^{-12}$ ).

\section{IV.2.3.2 Error propagation with Monte-Carlo simulation Method (MCM)}

With $M C M$, the errors in AGB estimation were propagated for the study area. The data from 47 measured plots $(\mathrm{DBH}, \mathrm{TH})$ was assumed as the best estimate per tree. Then, on these measurements were applied to the error measurement parameters of the NDn and $\bmod$ scenarios. These scenarios were selected since they were observed in the previous Sections to be the scenarios that best estimated the measurement errors for the $C B$ and $T V$ datasets. The student measurement error parameters (Exp and Nexp) were included in the propagation. Finally, two more scenarios: the bestfitted PDF $(B D)$ and the best-fitted PDF by class $(B D C)$ were added.

The error parameters were mentioned in the previous section, except for the methods in which errors were non-normal distributed $(B D$ and $B D C$ ). The error parameters of the remaining scenarios ( $C B B D, C B B D C$, TVBD, and TVBCD) are displayed in Tables VIII-4 to VIII-7 of Appendix III.

Table IV-11 shows the results of error propagation with $M C M$. This table includes the contribution in percentage of non-sampling errors $(u N S)$ and sampling error $(S E)$. Both contributions to the total uncertainty $(u W)$ in the AGB estimate $(W)$, as well as the relative standard error $(R S E)$.

For all analyzed scenarios, the greatest contribution came from $S E$ (>99.05\%). Using parameters from Caliper and Blume-Leiss (CB), the reference scenarios CBNDn and CBmod had the same contribution of $0.10 \%$ for $u N S$. The $C B B D$ scenarios underestimated $(0.07 \%)$ the contribution of errors due to $u N S$. In contrast, using error distributions according to the diameter class $(C B B D C)$, the contribution of $u N S$ was overestimated $(0.15 \%)$.

Error propagation using the Tape and Vertex (TV) parameters is reported in the lower part of Table IV-11. For this data, a higher contribution of the TVmod scenario $(0.05 \%)$ was observed compared 
to TVNDn $(0.03 \%)$. However, the TVBDC scenario had the same contribution of $u N S$ as the reference scenario TVNDn. Similarly, the TVBDC scenario contributed $0.05 \%$, as did TVmod.

The propagations using the errors estimated with the student measurement errors (Exp and Nexp) had a major contribution to the $u N S$. With contributions close to $1 \%$ of the $u N S$ to the $u W$; the estimated values resulted in an increase in $R S E$ from $5.00 \%$ to $5.04 \%$.

\begin{tabular}{|c|c|c|c|c|c|c|c|}
\hline Uncertainty & $N S$ & $S E$ & $\begin{array}{c}u^{2} \\
N S\end{array}$ & $S E^{2}$ & $\begin{array}{l}u \\
W\end{array}$ & $W$ & $R S E$ \\
\hline scenario & $M g h a^{-1}$ & $M g h a^{-1}$ & $\%$ & $\%$ & $M g h a^{-1}$ & $M g h a^{-1}$ & $\%$ \\
\hline CBNDn & 0.42 & 13.13 & 0.10 & 99.90 & 13.14 & 262.67 & 5.00 \\
\hline CBmod & 0.42 & 13.13 & 0.10 & 99.90 & 13.14 & 262.70 & 5.00 \\
\hline$C B B D$ & 0.35 & 13.13 & 0.07 & 99.93 & 13.14 & 262.69 & 5.00 \\
\hline$C B B D C$ & 0.50 & 13.14 & 0.15 & 99.85 & 13.15 & 262.67 & 5.00 \\
\hline TVNDn & 0.24 & 13.13 & $\mathbf{0 . 0 3}$ & 99.97 & 13.13 & 262.70 & 5.00 \\
\hline TVmod & 0.29 & 13.13 & 0.05 & 99.95 & 13.13 & 262.71 & 5.00 \\
\hline$T V B D$ & 0.23 & 13.13 & 0.03 & 99.97 & 13.13 & 262.71 & 5.00 \\
\hline$T V B D C$ & 0.30 & 13.13 & 0.05 & 99.95 & 13.13 & 262.71 & 5.00 \\
\hline TVExp & 1.27 & 13.18 & 0.93 & 99.07 & 13.24 & 262.67 & 5.04 \\
\hline TVNexp & 1.29 & 13.17 & 0.95 & 99.05 & 13.24 & 262.63 & 5.04 \\
\hline
\end{tabular}

$C B=$ Caliper + Blume-Leiss, $T V=$ Tape + Vertex,$N D n=$ normal distribution, mod=linear regression model, $B D=$ best fitted distribution, $B D C=$ best fitted distribution by class, Exp=students with experience, Nexp=students without experience.

Table IV-11. Error propagation in AGB estimation with $M C M$ different scenarios to calculate measurement errors.

The analyses to compare the mean uncertainty estimated at the plot level was performed. First, were compared the mean uncertainty from propagations using error estimates for Caliper+Blume-Leiss $(C B)$. The ANOVA of repeated measurements (Tables VIII-35 and VIII-36, Appendix V) was significant ( $\mathrm{p}$-value $\leq 2.2 \mathrm{e}^{-16}$ ). The paired T-Test found that the mean uncertainty in all applied scenarios were significantly different, with $\mathrm{p}$-value $\leq 3.2 \mathrm{e}^{-05}$.

Results of comparisons using Tape+Vertex (TV) mean uncertainty estimates were like the $C B$ results (Tables VIII-37 and 38, Appendix V). It was found a significant difference between the scenarios ( $\mathrm{p}$-value $\leq 1.603 \mathrm{e}^{-13}$ ), and the paired comparison determined that the mean uncertainty from the scenarios were significantly distinct ( $\mathrm{p}$-value $\leq 1.2 \mathrm{e}^{-08}$ ).

The mean uncertainty of propagation with error parameters by students (Exp,Nexp) with the reference measurements scenarios were compared (CBNDn, CBmod, TVNDn, and TVmod). The result of ANOVA (Table VIII-39 and VIII-40, Appendix V) revealed significant differences (pvalue $<2.2 \mathrm{e}^{-16}$ ). Post hoc analysis showed no significant difference between the mean uncertainty from the two scenarios with student information (TVExp and TVNexp), but the remainder of the pair analyses were statistically different ( $\mathrm{p}$-value $\leq 5.40 \mathrm{e}^{-05}$ ).

\section{IV.2.3.2.1 Contribution per source of uncertainty}

In the previous section, a major contribution of sampling error $(S E)$ to the total uncertainty $(u W)$ was found. Non-sampling uncertainty $(u N S)$ consisted of four sources of uncertainty, and it was split in the contribution of each source in this Section. When the contribution was separated, a 
contrast between the sources of $u N S$ was revealed among the different scenarios used to estimate measurement errors.

Out of the scenarios used in the previous Section, the NDn scenario was excluded because its estimates were not significantly different from the ones in mod scenario. Additionally, the mod scenario used the size of the tree in estimating measurement errors.

The error propagation results with $M C M$ separated by the source of uncertainty are displayed in Table VIII-41 of Appendix V. Using Equation 11, the contributions with units of $\mathrm{Mg} \mathrm{ha}^{-1}$ were obtained, and the Law of Error Propagation was used (Equation 15) to approximate the total $u N S$ for the scenario $B D C$.

(CB) Caliper + Blume-Leiss $0.50 \approx \sqrt{(0.501)^{2}+(0.202)^{2}+(0.453)^{2}+(0.008)^{2}+(0.018)^{2}}$

(TV) Tape + Vertex

$$
0.30 \approx \sqrt{(0.302)^{2}+(0.158)^{2}+(0.260)^{2}+(0.004)^{2}+(0.018)^{2}}
$$

The contributions by source of uncertainty showed that the two sources that contributed most to the total non-sampling uncertainty $(u N S)$ were $u D B H$ and $u T H$. Contributions from the other two sources, correlation $(<0.006 \%)$ and prediction model $(<0.0002 \%)$, were relatively small.

Figure IV-10 presents the contributions from $u D B H$ and $u T H$ to the $u N S$ estimates in Table VIII-41 of Appendix V. In the figure were included six scenarios, used in the previous section with the error propagation using $M C M$. The scenarios were constructed with two datasets ( $C B$ and $T V$ ) and tree methods to estimate measurement errors ( $\bmod , B D$ and $B D C)$.

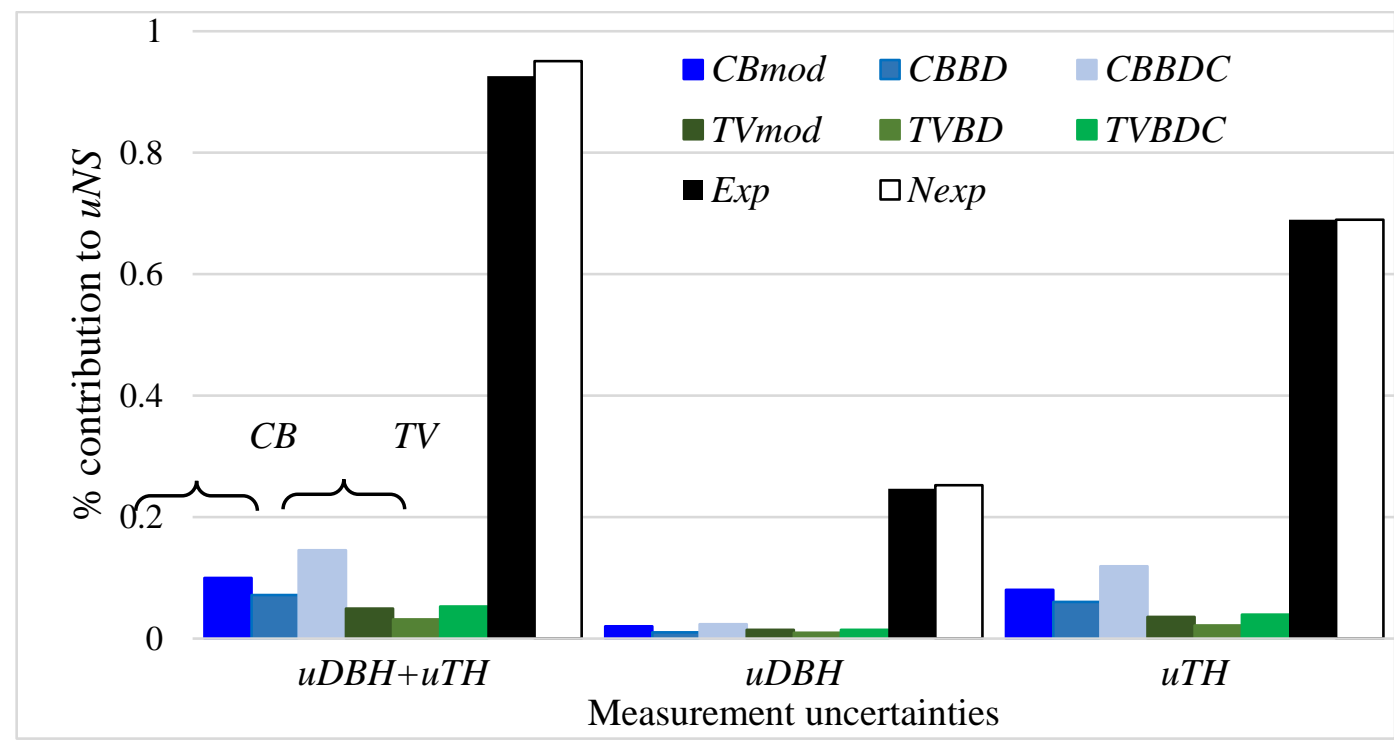

$u N S=$ total non-sampling uncertainty, $C B=$ Caliper + Blume-Leiss, $T V=$ Tape + Vertex , mod=linear regression model, $B D=$ best fitted distribution, $B D C=$ best fitted distribution by class.

Figure IV-10. Contribution of uncertainties from $D B H$ and $T H$ measurements to the total nonsampling uncertainty of AGB estimate.

The figure shows the overall contribution of the two uncertainties $(u D B H+u T H)$ to the total $N S$ uncertainty, for each scenario. The total $u N S$ had larger contributions from scenarios using 
Caliper+Blume-Leiss ( $C B \geq 0.072 \%$ ) compared to the contributions of scenarios with Tape+Vertex ( $T V \leq 0.053 \%$ ). The contribution to $u N S$ by student measurements (Exp and Nexp) was close to $1 \%$.

The $T H$ errors were the largest contributors to $u N S$. In the scenarios using $C B$ data, they represented more than $80 \%$ of the $u N S$, and they contributed to more than $68 \%$ for $T V$ scenarios. Applying errors from students' measurements (Exp and Nexp), TH error accounted for more than $72 \%$ of the uNS. On the other hand, measurement errors in $D B H$ were responsible for less than $28 \%$ of the errors in $u N S$.

\section{IV.2.3.2.2 Error contribution of AGB estimation by measurement device}

To analyze the differences in uncertainty estimation by a measurement device, the uncertainties were compared by pairs of devices (Tape vs. Caliper and Vertex vs. Blume-Leiss). This comparison with the mean uncertainty propagated at plot-level was performed for each of the scenarios used in the previous Section $(B D, B D C$, mod $)$. The boxplots in Figure IV-11 show the mean uncertainty estimates among measurement pairs, with $u D B H$ displayed on the left and $u T H$ on the right. The right margin describes the scenarios used to estimate measurement errors. The lines join the estimates of the same plot made by the two instruments being compared.

In the previous section was found that the uncertainties of $T H$ measurements were larger than those of $D B H$. For $u D B H$, Tape errors were smaller than Caliper errors for the three scenarios. However, the $B D$ and $B D C$ scenarios occasionally produced similar estimates for both devices. In Figure VIII2 of Appendix V, a complementary graph of the error estimates by plot and device was included.

For the $\mathrm{TH}$ measurement errors (graphs on the right side), the uncertainty estimates with BlumeLeiss measurements were greater than those observed in Vertex in all the estimates (see Figure VIII2, Appendix V).

To verify the graphical differences, a paired analysis for the mean $D B H$ uncertainties was performed. The Kolmogorov-Smirnov Test verified the normal distributions of the uncertainty (pvalue $_{K S} \geq 0.082$ ). Subsequently, a paired T-Test was applied (Holm's correction), and the results indicated that from the three scenarios, $D B H$ mean uncertainty estimates were statistically distinct ( $\mathrm{p}$-value $\left.\mathrm{t}_{\mathrm{t}-\mathrm{Holm}} \leq 2.2 \mathrm{e}^{-16}\right)$. The mean value of the differences was greatest in the $B D C$ scenario $(0.255$ $\left.\mathrm{Mg} \mathrm{ha}^{-1}\right)$, followed by $\bmod \left(0.188 \mathrm{Mg} \mathrm{ha}^{-1}\right)$, and then the $B D$ scenario $\left(0.028 \mathrm{Mg} \mathrm{ha}^{-1}\right)$.

The Kolmogorov-Smirnov's Test, when applied to the $T H$ measurements, resulted in a pvalue $_{\mathrm{Ks}} \geq 0.06$. The $T$-Tests also found statistically significant differences in the data pairs of uncertainties per plot ( $\mathrm{p}$-value $\mathrm{t}_{\text {-Holm }} \leq 2.2 \mathrm{e}^{-16}$ ). In ascending order, the mean value of the differences was $0.808 \mathrm{Mg} \mathrm{ha}^{-1}$ for scenario $\mathrm{mod}, 0.817 \mathrm{Mg} \mathrm{ha}^{-1}$ for $B D$ and $1.250 \mathrm{Mg} \mathrm{ha}^{-1}$ for $B D C$. 


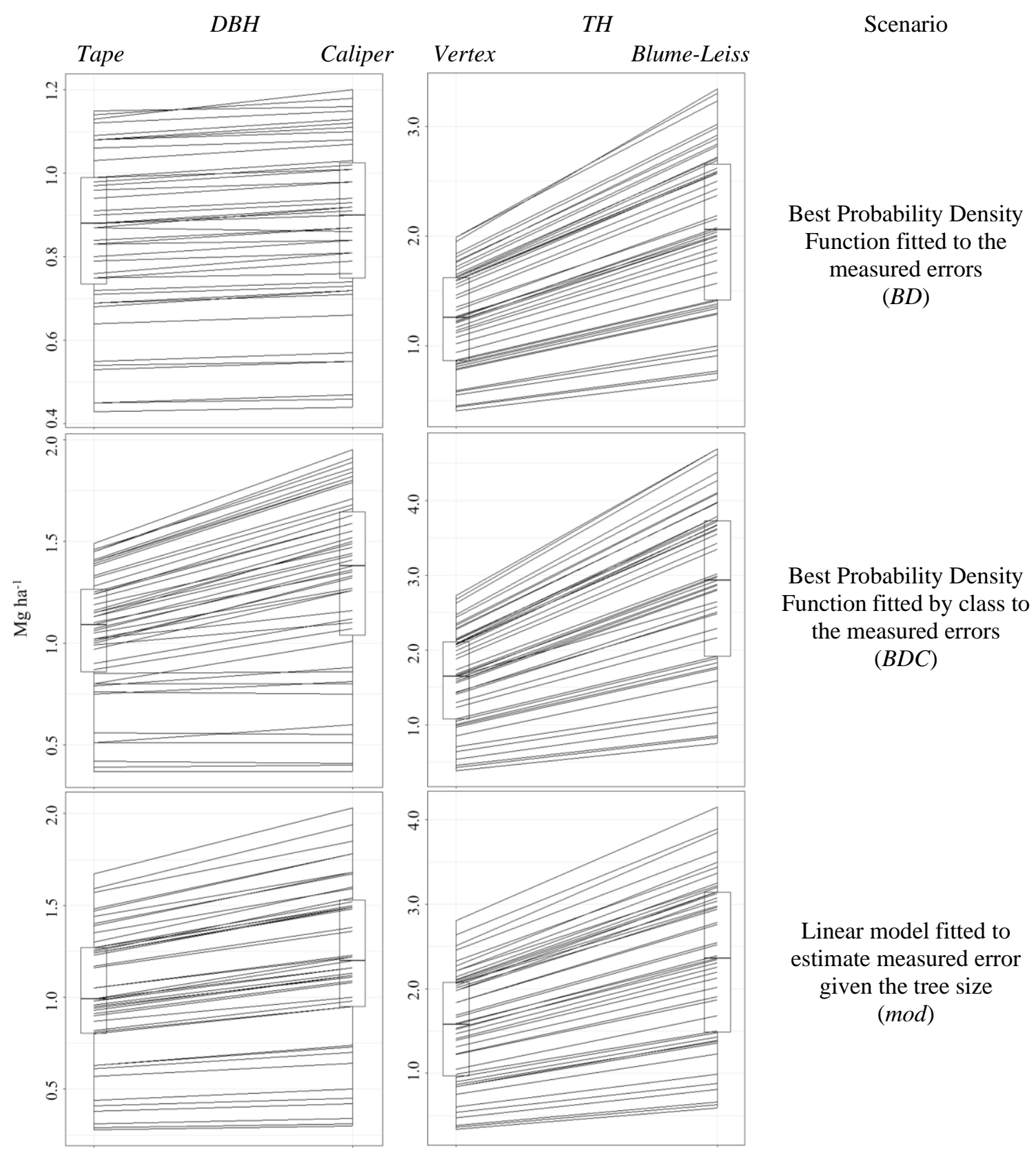

Figure IV-11. Comparison of uncertainty calculated per plot and measurement device, for three scenarios of measurement error in AGB estimation. 


\section{IV.3 Empirical study 3: Propagation of errors in the AGB estimation for the state of Durango, Mexico, with information from the National Forest Inventory}

\section{IV.3.1 General information on the Mexican National Forest Inventory dataset}

\section{IV.3.1.1 First measurement of the Mexican National Forest Inventory (2004-2009)}

The data used in this analysis derives from the Mexican National Forest Inventory (MNFI) from the years 2004 to 2009. The 2004-2009 MNFI records for the state of Durango consisted of 1826 clusters with 118,647 trees. Our study excluded 157 clusters ( 8320 records) because they were from a vegetation classification other than temperate forests. Errors detected in the records were "missing information" and "outliers". There were 73 missing information registers: 21 without $D B H, 46$ without $T H$, and 6 without a name registration. There were 492 outlier records, from which 141 had a $D B H$ less than $7.5 \mathrm{~cm}$ and 351 had a $T H$ less than $1.5 \mathrm{~m}$. These limits were established in the measurement protocol. The errors in this raw dataset were the $0.51 \%$ of the total trees.

Our final subset of data from the 2004-2009 MNFI in the temperate forest in Durango consisted of 1662 clusters with 109,762 trees. This dataset was used as reference information to estimate basal area $(G)$ and AGB $(W)$ from the estimators in Section III.3.5. The summary of these results is in Table IV-12, in which the standard error $(S E(\hat{R}))$ refers to the sampling error and $s(\hat{R})$ indicates the dispersion of the mean estimate per cluster.

\begin{tabular}{|c|c|c|c|c|c|c|c|}
\hline \multirow{2}{*}{ Vegetation } & \multirow{2}{*}{ Estimator } & \multicolumn{3}{|c|}{$\begin{array}{c}2004-2009 \\
\text { Clusters }=1662\end{array}$} & \multicolumn{3}{|c|}{$\begin{array}{c}2009-2014 \\
\text { Clusters }=1632\end{array}$} \\
\hline & & $\begin{array}{l}\text { Tree } \\
\text { No. }\end{array}$ & $\begin{array}{c}G \\
\mathrm{~m}^{2} \mathrm{ha}^{-1} \\
\end{array}$ & $\begin{array}{c}W \\
\mathrm{Mg} \mathrm{ha}^{-1}\end{array}$ & $\begin{array}{l}\text { Tree } \\
\text { No. }\end{array}$ & $\begin{array}{c}G \\
\mathrm{~m}^{2} \mathrm{ha}^{-1} \\
\end{array}$ & $\begin{array}{c}W \\
\mathrm{Mg} \mathrm{ha}^{-1} \\
\end{array}$ \\
\hline \multirow{3}{*}{ Temperate forest } & $\hat{R}$ & 445.16 & 12.98 & 64.31 & 451.76 & 13.17 & 64.77 \\
\hline & $s(\hat{R})$ & 279.16 & 8.56 & 77.80 & 278.36 & 8.66 & 77.70 \\
\hline & $S E(\widehat{R})$ & 7.10 & 0.22 & 1.93 & 7.13 & 0.22 & 1.94 \\
\hline
\end{tabular}

Table IV-12. Estimates of basal area $(G)$ and AGB $(W)$ calculated from the MNFI in the temperate forest of Durango, Mexico.

\section{IV.3.1.2 First re-measurement of MNFI (2009-2014)}

The information from the following MNFI was designated as "re-measurement" data. The 20092014 MNFI included 1822 clusters registered in Durango, with 118,641 measured trees. From the Durango subset, temperate forest in 1632 clusters were identified, thereby removing 8191 tree records from the other vegetation types. Additionally, 18 double records and 417 records without information were eliminated. Seven records were also eliminated with $D B H$ less than $7.5 \mathrm{~cm}$ and 25 trees with $T H$ less than $1.5 \mathrm{~m}$, per the established measurement protocol. The error in the dataset represented $0.40 \%$ of the trees measured. In the end, basal area and AGB were estimated for the Durango temperate forest with 109,983 records from the 2009-2014 dataset (Table IV-12).

The estimates of $G$ and $W$ increased from the 2004-2009 dataset to the 2009-2014 re-measurement dataset. The ratio estimate increased by seven trees $\mathrm{ha}^{-1}, 0.19 \mathrm{~m}^{2} \mathrm{ha}^{-1}$ for basal area, and $460 \mathrm{~kg} \mathrm{ha}^{-1}$ for AGB. In addition, the variation between plots was lower in the re-measurement for the number of trees and AGB but was higher for the basal area. Finally, the standard error showed no change for the basal area but the other two variables increased. 


\section{IV.3.2 AGB and basal area estimation by size classes}

The AGB $(W)$ and the basal area $(G)$ were estimated by $D B H$ and $T H$ class to determine the proportion of each class in the total estimate. The $W$ and $G$ estimates per class, for the 2004-2009 MNFI dataset (ALL), is in Figure IV-12. Since the genera Pinus spp (PINE) and Quercus spp (OAK) were dominant $(84.3 \%$ of the dataset), the estimate of both genera and the sum of the two genera were included $(P \& O) . D B H$ and $T H$ classes were outlined in Section III.1.5.1.1.1.

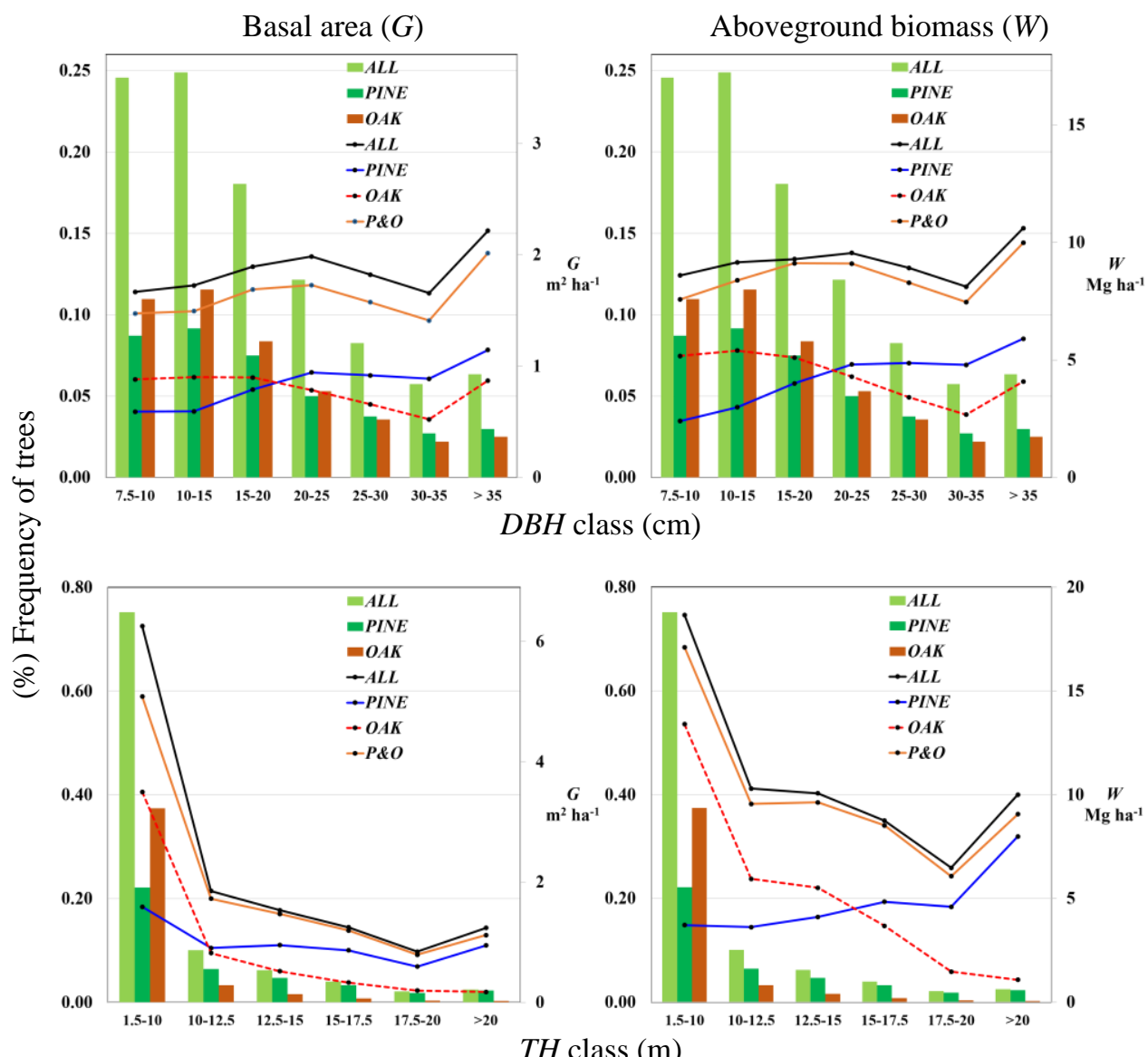

$A L L=$ estimation made with all the data of temperate forest, $P I N E=$ estimation made with genus Pinus spp $(\mathrm{n}=43531), O A K=$ estimation made with genus Quercus spp $(\mathrm{n}=48993), P \& O=$ estimation made with Pinus spp + Quercus spp $(\mathrm{n}=92524)$.

Figure IV-12. Basal area $(G)$ and AGB $(W)$ estimation for 2004-2009 MNFI in Durango, Mexico. Above, estimations made by $D B H$ class; below, estimations made by $T H$ class.

The upper part of Figure IV-12 shows the results of the estimation by $D B H$ class and in the lower part the estimates by $T H$ classes. The number of trees by class was represented with vertical bars according to the percentage of trees with respect to the total MNFI. the values of $W$ and $G$ were represented with connected points, to display the direction followed by the estimates. The information source for Figure IV-12 was Table VIII-42 of Appendix VI.

\section{IV.3.2.1 DBH classes}

In the diameter data, the frequency of trees per class was similar for the three datasets (ALL, PINE, and $O A K)$. The $D B H$ class with the highest frequency was $[10-15 \mathrm{~cm}$ ) for the three sets. The class that contained the mean for the three data sets was $[15-20 \mathrm{~cm}$ ), with means of $16.7 \mathrm{~cm}, 18.1 \mathrm{~cm}$, and 
$16.2 \mathrm{~cm}$, for $A L L, P I N E$, and $O A K$, respectively. In the four lower classes, $O A K$ had a higher frequency than PINE, and, conversely, in the three classes with larger $D B H$, the frequency of PINE was higher.

The class estimates from the dataset $A L L$ (the top line in the two graphs) had a similar contribution from the two variables. The estimates ranged from 1.66 to $2.22 \mathrm{~m}^{2} \mathrm{ha}^{-1}$ and from 8.12 to $9.55 \mathrm{Mg}$ $\mathrm{ha}^{-1}$ for $G$ and $W$. The graphs demonstrate that the frequency of trees in the small tree classes was higher. However, the four larger tree classes with $32.5 \%$ of the trees contributed to $59.2 \%$ of $G$ and $57.9 \%$ of $W$.

The PINE $(\mathrm{n}=43531)$ and $O A K(\mathrm{n}=48993)$ contributions were inversely proportional. As the PINE contribution increased, the $O A K$ contribution decreased. This trend was observed up to the class [30-35 cm), except for the last class in which both groups increased their contribution. The contribution of the $P \& O$ dataset (sum of PINE and $O A K$ ) is observed under and parallel to the $A L L$ contribution. $P \& O$ contributed to $87.7 \%$ of the total $G$ estimate and $93.2 \%$ of the total $W$ estimate.

\section{IV.3.2.2 TH classes}

The lower part of Figure IV-12 shows the estimated results of $G$ and $W$ by $T H$ class. The figure shows that the class with the smallest tree height (vertical bars) had the highest frequency, with most trees being of the genus Quercus spp $(O A K)$. The frequency generally decreased as $T H$ increased, except for the last class. The lowest class was the class containing the mean for the datasets of $A L L$ and $O A K$, with values of $7.7 \mathrm{~m}$ and $6.2 \mathrm{~m}$. The class of the mean for PINE was [1012.5), with $10.1 \mathrm{~m}$.

The contribution to $G$ was highest in the lowest $T H$ class. In this class, $O A K$ had the highest contribution $\left(3.49 \mathrm{~m}^{2} \mathrm{ha}^{-1}\right)$, while the rest of the classes had the largest contribution from PINE. $O A K$ 's contribution to $W$ decreased with increasing tree size, while PINE gradually increased its contribution from trees larger than 10 meters. As a result, $45.3 \%$ of PINE greater than 10 meters contributed to $87.1 \%$ of the estimated $W$ for this genus. In contrast, for the same $T H$ range, the $14.2 \%$ of $O A K$ contributed to only $56.9 \%$ of the $W$.

The estimation of $G$ and $W$ with the Pine \& Oak dataset was already included in the previous Section. However, it is worth noting that for the estimate of $G$, a smaller contribution was observed from the smallest $T H$ class, i.e. the shortest trees. In this class, $P \& O$ 's contribution was $81.1 \%$, and, in the remaining classes, it was greater than $90.5 \%$. The $P \& O$ contribution to the $W$ estimate was greater than $90 \%$ in all classes.

The 2009-2014 MNFI data, analyzed by classes of $D B H$ and $T H$, showed a similar behavior as described for the first MNFI period. Since the description of these results does not represent a relevant contribution, the graphs of the second MNFI period can be found in Appendix VI for reference (Figure VIII-3).

\section{IV.3.3 Error propagation of AGB}

The error in the estimate of AGB was propagated with $M C M$ from the individual sources of error to the total error. Field variable measurement error (uMes), AGB predictions with allometric models $(u A M)$, and plot design (uPlot) were the identified sources of error, besides $S E$. The random errors 
were incorporated into non-sampling sources of uncertainty (uMes and $u A M)$ through three scenarios.

In the first scenario $(B D)$, the assumption was that $u M e s$ came from a single probability distribution $(P D F)$ of each measured variable $(D B H$ and $T H)$. For the second scenario $(B D C)$, an increase of measurement error as the class of the measured variable increased (heteroscedasticity) was assumed. The third scenario (Exp) estimated the uMes with the parameters applied to the students' experience in mensuration (from the second empirical study). In this scenario, it was assumed that uMes by students were under similar conditions as MNFI.

Measurement uncertainty parameters for the $B D$ and $B D C$ scenarios developed in Empirical Study 1 are reported in Tables VIII-2 and VIII-3 of Appendix II. For the Exp scenario used in Empirical Study 2, Table IV-6 shows the measurement error parameters. Furthermore, the allometric models and their uncertainty parameters are in Table VIII-1 of Appendix I. Finally, the error parameter of the plot design was described in Section III.3.8.3.

The information for both analyzed datasets with the acronyms $M N F I_{0409}$ for the 2004-2009 MNFI

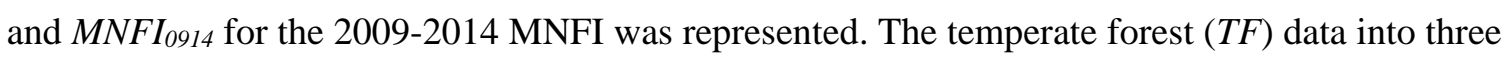
strata was divided, each defined by the dominant vegetation. Those strata were conifer forest $(S C F)$, mixed forest $(S M F)$, and oak forest $(S O F)$. Similarly, each stratum had two substrata to define the dominance of primary vegetation $(p)$ or secondary vegetation $(s)$.

It is important to make a note about the number of clusters in each strata and substrata since they were the primary sampling units in the MNFI. Thus, the tables of results for strata and substrata always reported the number of clusters. This number refers to how many clusters in the temperate forest have at least one site identified with that stratum and/or substratum. Because every cluster can have more than one stratum, when adding the clusters of the three strata and substrata, the result is not equal to the total number of temperate forest clusters.

\section{IV.3.3.1 Temperate forest of Durango, Mexico}

The error propagation, estimated for the two periods under study, is in Table IV-13. The result of the total estimate $(W)$, as well as the total uncertainty $(u W)$, is given in the measurement units. Uncertainty contributions (non-sampling error - $u N S$ - and sampling error -SE-) are given in percentage, as was the relative standard error (RSE). The result of $u W$ is reported to three significant figures to see the differences between the scenarios. The number of clusters used in the estimates was $\mathrm{n}=1662$ for $M N F I_{0409}$ and $\mathrm{n}=1632$ for $M N F I_{0914}$.

The uncertainty of the baseline result (bold line) was $1927 \mathrm{Mg} \mathrm{ha}^{-1}$ for $M N F I_{0409}$ and $1940 \mathrm{Mg} \mathrm{ha}^{-1}$ for $M N F I_{0914}$. This uncertainty was estimated only with the $S E$. Once was calculated the ratio $u W / W$, it was found that $R S E(3.00 \%)$ was equal in both MNFI periods. In a step before, non-sampling uncertainties $(u N S)$ were less than $1 \%$ of the total uncertainty $(u W)$ in both periods. In this case, the scenario with the highest contribution to $u W$ was TFExp (0.71\%), followed by TFBDC, and the lowest contribution was in TFBD. Notably, the SE contributed more than $99 \%$ to the $u W$ in AGB estimates.

With the $M C M$, the confidence interval for the estimated $u W$ with $95 \%$ reliability was calculated. Besides, the interval across the lower $(L L)$ and upper $(U L)$ limits of $R S E$ was included in the table. 
Finally, non-differences were observed between the estimated confidence limits for the three scenarios in both periods.

The difference in the estimates of the mean value $(W)$ met the criterion set out in Section III.1.5.3. This criterion established the difference between scenario $W$ (TFBD, TFBDF, TFExp) and the reference $W(T F)$ to be less than $1 \%$. In the temperate forest, this difference was less than $0.1 \%$.

\begin{tabular}{|c|c|c|c|c|c|c|c|c|c|c|c|c|c|c|}
\hline \multirow{3}{*}{$\begin{array}{c}\text { Uncertainty } \\
\text { source }\end{array}$} & \multicolumn{7}{|c|}{ MNFI 2004-2009 $\left(\mathrm{n}_{0409}=1662\right)$} & \multicolumn{7}{|c|}{ MNFI 2009-2014 (n0914=1632) } \\
\hline & $\begin{array}{c}u^{2} \\
N S\end{array}$ & $S E^{2}$ & $\begin{array}{l}u \\
W\end{array}$ & $W$ & $\begin{array}{c}R S E \\
L L\end{array}$ & $R S E$ & $\begin{array}{c}R S E \\
U L\end{array}$ & $\begin{array}{c}u^{2} \\
N S\end{array}$ & $S E^{2}$ & $\begin{array}{l}u \\
W\end{array}$ & $W$ & $\begin{array}{c}R S E \\
L L\end{array}$ & $R S E$ & $\begin{array}{c}R S E \\
U L\end{array}$ \\
\hline & $\%$ & $\%$ & $M g_{I} h a^{-}$ & $\operatorname{Mg}_{l} h a^{-}$ & $\%$ & $\%$ & $\%$ & $\%$ & $\%$ & $M g_{I} h a^{-}$ & $\operatorname{Mg}_{l} h a^{-}$ & $\%$ & $\%$ & $\%$ \\
\hline$T F$ & & 100.00 & 1.927 & 64.31 & & 3.00 & & & 100.00 & 1.940 & 64.77 & & 3.00 & \\
\hline$T F B D$ & 0.55 & 99.45 & 1.932 & 64.31 & 2.98 & 3.00 & 3.03 & 0.56 & 99.44 & 1.946 & 64.77 & 2.98 & 3.00 & 3.03 \\
\hline$T F B D C$ & 0.59 & 99.41 & 1.934 & 64.36 & 2.98 & 3.00 & 3.03 & 0.59 & 99.41 & 1.948 & 64.82 & 2.98 & 3.00 & 3.03 \\
\hline TFExp & 0.71 & 99.29 & 1.931 & 64.28 & 2.98 & 3.00 & 3.03 & 0.67 & 99.33 & 1.945 & 64.75 & 2.98 & 3.00 & 3.03 \\
\hline
\end{tabular}

Table IV-13. Error propagation in AGB estimation using three scenarios of non-sampling uncertainty in temperate forest of Durango, Mexico.

By converting the results of $u N S(0.59 \%)$ and $S E(99.41 \%)$ into $\mathrm{Mg} \mathrm{ha}^{-1}$ and substituting into Equation 11, the total uncertainty for the period 2004-2009 for the BDC scenario was estimated: $u W$ $\approx 1.934 \approx \sqrt{(1.929)^{2}+(0.15)^{2}}$

\section{IV.3.3.1.1 Strata in the temperate forest of Durango, Mexico}

The AGB estimates were calculated for the temperate forest strata and reported the results in Table IV-14. As with the complete temperate forest dataset, the AGB for each stratum was estimated (bold rows). This table includes in the first column, the number of clusters per strata for both periods of study. Here, it was observed that the mixed forest $(S M F)$ had the highest AGB per ha in the two periods $\left(\geq 73.123 \mathrm{Mg} \mathrm{ha}^{-1}\right)$. Furthermore, the highest total uncertainty due to sampling was estimated in the conifer forest ( $S C F$ ) of $M N F I_{0914}$, with $10.62 \%$ of $R S E$.

The results by scenario showed that $u N S$ for $S C F$ and $S M F$ were about $0.67 \%$ of the total uncertainty. A greater contribution was observed of $u N S$ in the oak forest (SOF), ranging from 1.95 to $2.51 \%$ of the $u W$. Moreover, the $u N S$ contributions increased the estimated uncertainties in SCF and $S M F$ from less than 0.01 to $0.02 \mathrm{Mg} \mathrm{ha}^{-1}$, while in $S O F$ it increased from 0.02 to $0.03 \mathrm{Mg} \mathrm{ha}^{-1}$. The $S E$ contributed to more than $99.3 \%$ of the total uncertainty of $S C F$ and $S M F$, while the contribution in SOF ranged from 97.49 to $98.05 \%$.

The $B D$ scenario was the most conservative in estimating $u N S$, followed by $B D C$. The Exp scenario had the highest contributions of $u N S$ out of all strata. Comparing the two periods, in $M N F I_{0914}$, the clusters in the strata decreased by $51 \%$ for $S C F, 7 \%$ for $S M F$, and $44 \%$ for $S O F$.

The errors propagated were compared by scenario by the paired T-Tests with Holm's correction. The assumption of normality was verified with the Kolmogorov-Smirnov Test with Lilliefor's correction, (p-value ${ }_{\mathrm{KS}} \geq 0.1216$ ). During the $M N F I_{0409}$ period, the uncertainties calculated by the $\operatorname{Exp}$ scenario were statistically different from those calculated for the $B D$ scenario ( $\mathrm{p}$-value $\mathrm{t}_{\mathrm{t}}=0.02658$ ). Other comparisons of this same period were not significantly different (p-value $\mathrm{t}_{\mathrm{tH}} \geq 0.0508$ ). No 
significant differences were found in the scenarios estimated at the level of strata for the $M N F I_{0914}$ data (p-value $\left.\mathrm{tH}_{\mathrm{H}} \geq 0.1068\right)$.

\begin{tabular}{|c|c|c|c|c|c|c|c|c|c|c|c|c|c|c|}
\hline \multirow{3}{*}{$\begin{array}{c}\text { Uncertainty } \\
\text { source }\end{array}$} & \multicolumn{7}{|c|}{ 2004-2009 } & \multicolumn{7}{|c|}{ 2009-2014 } \\
\hline & $\begin{array}{c}u^{2} \\
N S\end{array}$ & $S E^{2}$ & $\begin{array}{c}u \\
W\end{array}$ & $W$ & $\begin{array}{c}R S E \\
L L\end{array}$ & $R S E$ & $\begin{array}{c}R S E \\
U L\end{array}$ & $\begin{array}{c}u^{2} \\
N S\end{array}$ & $S E^{2}$ & $\begin{array}{l}u \\
W\end{array}$ & $W$ & $\begin{array}{c}R S E \\
L L\end{array}$ & $R S E$ & $\begin{array}{c}R S E \\
U L\end{array}$ \\
\hline & $\%$ & $\%$ & $\begin{array}{l}M g \\
h a^{-1}\end{array}$ & $\begin{array}{l}M g \\
h a^{-1}\end{array}$ & $\%$ & $\%$ & $\%$ & $\%$ & $\%$ & $\begin{array}{l}M g \\
h a^{-1}\end{array}$ & $\begin{array}{l}M g \\
h a^{-1}\end{array}$ & $\%$ & $\%$ & $\%$ \\
\hline $\begin{array}{l}\boldsymbol{S C F} \\
\mathrm{n}_{0409}=537 \\
\mathrm{n}_{0914}=264 \\
\boldsymbol{S M F}\end{array}$ & & 100.00 & 3.86 & 48.31 & & 8.00 & & & 100.00 & 5.49 & 51.82 & & 10.62 & \\
\hline $\begin{array}{l}\mathrm{n}_{0409}=1281 \\
\mathrm{n}_{0914}=1190\end{array}$ & & 100.00 & 2.79 & 77.48 & & 3.60 & & & 100.00 & 2.45 & 73.12 & & 3.36 & \\
\hline $\begin{array}{l}\mathrm{n}_{0409}=526 \\
\mathrm{n}_{0914}=295\end{array}$ & & 100.00 & 2.09 & 35.52 & & 5.96 & & & 100.00 & 2.40 & 36.84 & & 6.59 & \\
\hline$S C F B D$ & 0.46 & 99.54 & 3.87 & 48.33 & 7.90 & 8.00 & 8.10 & 0.33 & 99.67 & 5.51 & 51.84 & 10.48 & 10.62 & 10.78 \\
\hline$S M F B D$ & 0.48 & 99.52 & 2.79 & 77.49 & 3.58 & 3.60 & 3.63 & 0.56 & 99.44 & 2.46 & 73.13 & 3.33 & 3.36 & 3.38 \\
\hline$S O F B D$ & 2.26 & 97.74 & 2.12 & 35.50 & 5.84 & 5.96 & 6.08 & 1.95 & 98.05 & 2.43 & 36.82 & 6.43 & 6.59 & 6.75 \\
\hline$S C F B D C$ & 0.49 & 99.51 & 3.87 & 48.37 & 7.90 & 8.00 & 8.11 & 0.38 & 99.62 & 5.51 & 51.88 & 10.47 & 10.63 & 10.79 \\
\hline$S M F B D C$ & 0.51 & 99.49 & 2.80 & 77.55 & 3.57 & 3.60 & 3.64 & 0.56 & 99.44 & 2.46 & 73.18 & 3.33 & 3.36 & 3.39 \\
\hline$S O F B D C$ & 2.26 & 97.74 & 2.12 & 35.53 & 5.84 & 5.96 & 6.08 & 2.07 & 97.93 & 2.43 & 36.86 & 6.43 & 6.59 & 6.74 \\
\hline SCFExp & 0.59 & 99.41 & 3.88 & 48.42 & 7.88 & 8.00 & 8.13 & 0.47 & 99.53 & 5.52 & 51.93 & 10.44 & 10.62 & 10.81 \\
\hline SMFExp & 0.60 & 99.40 & 2.79 & 77.45 & 3.57 & 3.60 & 3.64 & 0.67 & 99.33 & 2.45 & 73.10 & 3.33 & 3.36 & 3.39 \\
\hline SOFExp & 2.51 & 97.49 & 2.11 & 35.58 & 5.83 & 5.96 & 6.09 & 2.22 & 97.78 & 2.42 & 36.72 & 6.43 & 6.59 & 6.75 \\
\hline
\end{tabular}

Table IV-14. Error propagation in AGB estimation, using three scenarios of non-sampling uncertainty in strata of the temperate forest of Durango, Mexico.

\section{IV.3.3.1.2 Substrata in the temperate forest of Durango, Mexico}

The reference values for the error propagation and AGB estimation by substratum are in Table IV15. In the reference values, it was observed that substrata of primary vegetation $(p)$ in the conifer forest $(S C F)$ and mixed forest $(S M F)$ strata, had higher $W$ estimations than the secondary vegetation substrate $(s)$. In contrast, the substratum of secondary vegetation in oak forest (SOFs) had a higher $W$ estimate than the primary vegetation $(S O F p$ ). The $S M F p$ substratum contained the highest AGB per unit area of $78.07 \mathrm{Mg} \mathrm{ha}^{-1}$ and $76.50 \mathrm{Mg} \mathrm{ha}^{-1}$ for the $M N F I_{0409}$ and $M N F I_{0914}$, respectively. The maximum uncertainty totaled to $9.62 \mathrm{Mg} \mathrm{ha}^{-1}$ in $S M F s$, and the minimum equaled $2.07 \mathrm{Mg} \mathrm{ha}^{-1}$ in $S O F p$. Proportional to the AGB estimate, the relative error (RSE) ranged between 3.6 and $23.5 \%$.

The error propagation, including the non-sampling uncertainty $(u N S)$ from the applied scenarios, is shown in Table IV-16. The total uncertainty $(u W)$ ranged from 2.09 to $9.68 \mathrm{Mg} \mathrm{ha}^{-1}$ in the $M N F I_{0409}$. Within the same stratum, a higher $u W$ was recorded in the substratum of secondary vegetation compared to the substratum with primary vegetation (secondary>primary). On the contrary, in $M N F I_{0914}$, greater $u W$ in the primary vegetation substratum (primary > secondary) was estimated. The uncertainty in this last period ranged from 3.15 to $8.66 \mathrm{Mg} \mathrm{ha}^{-1}$. The $u N S$ had a greater contribution to SOFp, with values from 2.83 to $3.61 \%$ of the $u W$. 


\begin{tabular}{|c|c|c|c|c|c|c|c|c|}
\hline & & & \multicolumn{3}{|c|}{ 2004-2009 } & \multicolumn{3}{|c|}{ 2009-2014 } \\
\hline & \multirow{2}{*}{\multicolumn{2}{|c|}{$\begin{array}{l}\text { Vegetation \& } \\
\text { cluster number }\end{array}$}} & $u W$ & $W$ & $R S E$ & $u W$ & $W$ & $R S E$ \\
\hline & & & $M g h a^{-1}$ & $M g h a^{-1}$ & $\%$ & $M g h a^{-1}$ & $M g h a^{-1}$ & $\%$ \\
\hline$S C F p$ & $\mathrm{n}_{0409}=500$ & $\mathrm{n}_{0914}=81$ & 4.17 & 50.12 & 8.3 & 8.64 & 59.57 & 14.5 \\
\hline SCFs & $\mathrm{n}_{0409}=60$ & $\mathrm{n}_{0914}=187$ & 7.21 & 30.81 & 23.5 & 6.84 & 47.77 & 14.3 \\
\hline$S M F p$ & $\mathrm{n}_{0409}=1261$ & $\mathrm{n}_{0914}=541$ & 2.85 & 78.08 & 3.7 & 3.73 & 76.51 & 4.9 \\
\hline SMFS & $\mathrm{n}_{0409}=60$ & $\mathrm{n}_{0914}=673$ & 9.62 & 58.83 & 16.4 & 3.26 & 70.19 & 4.7 \\
\hline SOFp & $\mathrm{n}_{0409}=476$ & $\mathrm{n}_{0914}=88$ & 2.07 & 35.44 & 5.9 & 3.43 & 35.80 & 9.7 \\
\hline SOFs & $\mathrm{n}_{0409}=86$ & $\mathrm{n}_{0914}=210$ & 5.78 & 36.01 & 16.2 & 3.14 & 37.36 & 8.5 \\
\hline
\end{tabular}

$S C F=$ conifer forest, $S M F=$ mixed forest, $S O F=$ oak forest, $p=$ primary vegetation, $s=$ secondary vegetation, $B D=$ best fitted distribution, $B D C=$ best fitted distribution by class, $\operatorname{Exp}=$ students.

Table IV-15. Reference values at the substratum level with the sampling error as the source of uncertainty in AGB estimation of the temperate forests in Durango, Mexico.

\begin{tabular}{|c|c|c|c|c|c|c|c|c|c|c|c|c|c|c|}
\hline \multirow{3}{*}{$\begin{array}{l}\text { Uncertainty } \\
\text { source }\end{array}$} & \multicolumn{7}{|c|}{ 2004-2009 } & \multicolumn{7}{|c|}{ 2009-2014 } \\
\hline & $\begin{array}{c}u^{2} \\
N S\end{array}$ & $S E^{2}$ & $\begin{array}{c}u \\
W\end{array}$ & $W$ & $\begin{array}{c}R S E \\
L L\end{array}$ & $R S E$ & $\begin{array}{c}R S E \\
U L\end{array}$ & $\begin{array}{c}u^{2} \\
N S\end{array}$ & $S E^{2}$ & $\begin{array}{l}u \\
W\end{array}$ & $W$ & $\begin{array}{c}R S E \\
L L\end{array}$ & $R S E$ & $\begin{array}{c}R S E \\
U L\end{array}$ \\
\hline & $\%$ & $\%$ & $M g h a^{-1}$ & $\begin{array}{l}M g \\
h a^{-1}\end{array}$ & $\%$ & $\%$ & $\%$ & $\%$ & $\%$ & $M g h a^{-1}$ & $\begin{array}{l}M g \\
h a^{-1}\end{array}$ & $\%$ & $\%$ & $\%$ \\
\hline$S C F p B D$ & 0.44 & 99.56 & 4.17 & 50.13 & 8.2 & 8.3 & 8.4 & 0.41 & 99.59 & 8.65 & 59.60 & 14.2 & 14.5 & 14.8 \\
\hline$S C F S B D$ & 1.14 & 98.86 & 7.24 & 30.80 & 22.5 & 23.5 & 24.5 & 0.33 & 99.67 & 6.85 & 47.78 & 14.0 & 14.3 & 14.6 \\
\hline$S M F p B D$ & 0.47 & 99.53 & 2.86 & 78.08 & 3.6 & 3.6 & 3.6 & 0.51 & 99.49 & 3.73 & 76.51 & 4.8 & 4.8 & 4.9 \\
\hline$S M F s B D$ & 0.96 & 99.04 & 9.66 & 58.81 & 15.8 & 16.4 & 16.9 & 0.58 & 99.42 & 3.26 & 70.19 & 4.6 & 4.6 & 4.7 \\
\hline$S O F p B D$ & 2.84 & 97.16 & 2.10 & 35.41 & 5.8 & 5.9 & 6.0 & 3.49 & 96.51 & 3.48 & 35.78 & 9.2 & 9.7 & 10.2 \\
\hline$S O F S B D$ & 1.24 & 98.76 & 5.81 & 36.00 & 15.6 & 16.1 & 16.7 & 1.57 & 98.43 & 3.16 & 37.34 & 8.2 & 8.4 & 8.6 \\
\hline$S C F p B D C$ & 0.48 & 99.52 & 4.18 & 50.17 & 8.2 & 8.3 & 8.4 & 0.46 & 99.54 & 8.66 & 59.62 & 14.2 & 14.5 & 14.8 \\
\hline$S C F s B D C$ & 1.17 & 9 & 7.25 & 30.84 & 22.5 & 23.5 & 24.5 & 0.36 & 64 & 6.86 & 47.82 & 14.0 & 14.3 & 14.6 \\
\hline$S M F p B D C$ & 0.50 & 99.50 & 2.86 & 78.14 & 3.6 & 3.6 & 3.7 & 0.53 & 99.47 & 3.74 & 76.57 & 4.8 & 4.8 & 4.9 \\
\hline$S M F s B D C$ & 0 & 99.02 & 9.68 & 58.87 & 15.8 & 16 & 17.0 & 0.60 & 99 & 3.27 & 70.24 & 4.6 & 4.6 & 4.7 \\
\hline$S O F p B D C$ & 2.83 & 97.17 & 2.10 & 35.44 & 5.8 & 5.9 & 6.0 & 3.48 & 96.52 & 3.49 & 35.81 & 9.2 & 9.7 & 10.2 \\
\hline$S O F s B D C$ & 1.19 & 98.81 & 5.82 & 36.02 & 15.5 & 16.1 & 16.7 & 1.59 & 98.41 & 3.16 & 37.38 & 8.2 & 8.4 & 8.6 \\
\hline SCFpExp & 0.56 & 99.44 & 4.18 & 50.22 & 8.2 & 8.3 & 8.4 & 0.59 & 99.41 & 8.66 & 59.68 & 14.1 & 14.5 & 14.8 \\
\hline SCFsExp & 1.33 & 98.67 & 7.26 & 30.86 & 22.5 & 23.5 & 24.6 & 0.45 & 99.55 & 6.87 & 47.87 & 14.0 & 14.3 & 14.6 \\
\hline SMFpExp & 0.60 & 99.40 & 2.85 & 78.03 & 3.6 & 3.6 & 3.7 & 0.65 & 99.35 & 3.73 & 76.47 & 4.8 & 4.8 & 4.9 \\
\hline SMFsExp & 1.19 & 98.81 & 9.67 & 58.81 & 15.8 & 16.4 & 17.0 & 0.67 & 99.33 & 3.26 & 70.17 & 4.6 & 4.6 & 4.7 \\
\hline SOFpExp & 3.15 & 96.85 & 2.09 & 35.27 & 5.8 & 5.9 & 6.0 & 3.61 & 96.39 & 3.47 & 35.65 & 9.2 & 9.7 & 10.2 \\
\hline SOFsExp & 0.62 & 99.38 & 5.81 & 35.97 & 15.5 & 16.1 & 16.8 & 1.76 & 98.24 & 3.15 & 37.26 & 8.2 & 8.4 & 8.6 \\
\hline
\end{tabular}

$S C F=$ conifer forest, $S M F=$ mixed forest, $S O F=$ oak forest, $p=$ primary vegetation, $s=$ secondary vegetation, $B D=$ best fitted distribution, $B D C=$ best fitted distribution by class, Exp=students.

Table IV-16. Error propagation in AGB estimation, using three scenarios ( $B D, B D C, \operatorname{Exp})$ of nonsampling uncertainty estimation in the study area.

For the three scenarios, the widest $R S E$ intervals ( $R S E L L$ and $R S E U L)$ were those with the smallest estimate of $W$. For $M N F I_{0409}$, this case was the substratum $S C F s$, and for $M N F I_{0914}$, it was the $S O F p$.

Substrata mean uncertainties were compared with a paired T-Test (Holm correction). During the $M N F I_{0409}$ period, $B D$ and $B D C$ scenarios were not significantly different $\left(\mathrm{p}\right.$-value $\left.\mathrm{t}_{\mathrm{tH}}=0.3009\right)$. The 
other pairs of comparisons, including the $\mathrm{MNFI}_{0914}$ comparisons, were significantly different, with a p-value ${ }_{t H} \leq 0.039$.

\section{IV.3.3.2 Contribution of the sources of uncertainty in AGB estimation}

The contribution $(\%)$ of the sources of uncertainty to the total uncertainty $(u W)$ of each scenario, is in Table IV-17. Here, were represented the sources of uncertainty $(u)$ with the letters $a$ for the uncertainty in $D B H, b$ for the uncertainty in $T H, c$ for the prediction from the allometric model, and $d$ for the uncertainty of plot design. The sum of contributions from these sources $(u N S)$ are shown in Table IV-13 in the reference values of each scenario.

\begin{tabular}{|c|c|c|c|c|c|c|c|c|c|c|c|c|c|c|}
\hline \multirow{3}{*}{$\begin{array}{l}\text { Uncertainty } \\
\text { source }\end{array}$} & \multicolumn{7}{|c|}{$2004-2009\left(\mathrm{n}_{0409}=1662\right)$} & \multicolumn{7}{|c|}{$2009-2014\left(\mathrm{n}_{0914}=1632\right)$} \\
\hline & $\begin{array}{c}u^{2} \\
N S\end{array}$ & $S E^{2}$ & $\begin{array}{l}u \\
W\end{array}$ & $\boldsymbol{W}$ & $\begin{array}{c}R S E \\
L L\end{array}$ & $R S E$ & $\begin{array}{c}R S E \\
U L \\
\end{array}$ & $\begin{array}{c}u^{2} \\
N S\end{array}$ & $S E^{2}$ & $\begin{array}{l}u \\
W\end{array}$ & $\boldsymbol{W}$ & $\begin{array}{c}R S E \\
L L \\
\end{array}$ & $R S E$ & $\begin{array}{c}R S E \\
U L \\
\end{array}$ \\
\hline & $\%$ & $\%$ & $\underset{1}{\mathrm{Mg} \mathrm{ha}^{-}}$ & $\operatorname{Mg~ha}_{1}^{-}$ & $\%$ & $\%$ & $\%$ & $\%$ & $\%$ & $\underset{1}{\operatorname{Mg} \mathrm{ha}^{-}}$ & $\underset{1}{\operatorname{Mg} \mathbf{h a}^{-}}$ & $\%$ & $\%$ & $\%$ \\
\hline$T F B D a$ & 0.00 & 100.00 & 1.927 & 64.34 & 2.99 & 3.00 & 3.00 & 0.00 & 100.00 & 1.940 & 64.80 & 2.99 & 3.00 & 3.00 \\
\hline$T F B D b$ & 0.01 & 99.99 & 1.926 & 64.28 & 2.99 & 3.00 & 3.00 & 0.01 & 99.99 & 1.940 & 64.74 & 2.99 & 3.00 & 3.00 \\
\hline$T F B D c$ & 0.52 & 99.48 & 1.932 & 64.31 & 2.99 & 3.00 & 3.02 & 0.53 & 99.57 & 1.945 & 64.77 & 2.98 & 3.00 & 3.02 \\
\hline$T F B D d$ & 0.02 & 99.98 & 1.927 & 64.31 & 2.99 & 3.00 & 3.00 & 0.02 & 99.98 & 1.940 & 64.77 & 2.99 & 3.00 & 3.00 \\
\hline$T F B D C a$ & 0.01 & 99.99 & 1.929 & 64.37 & 2.99 & 3.00 & 3.00 & 0.01 & 99.99 & 1.942 & 64.83 & 2.99 & 3.00 & 3.00 \\
\hline$T F B D C b$ & 0.03 & 99.97 & 1.927 & 64.30 & 2.99 & 3.00 & 3.00 & 0.03 & 99.97 & 1.940 & 64.76 & 2.99 & 3.00 & 3.00 \\
\hline$T F B D C c$ & 0.53 & 99.47 & 1.932 & 64.31 & 2.98 & 3.00 & 3.02 & 0.53 & 99.47 & 1.945 & 64.77 & 2.98 & 3.00 & 3.02 \\
\hline$T F B D C d$ & 0.02 & 99.98 & 1.927 & 64.31 & 2.99 & 3.00 & 3.00 & 0.02 & 99.98 & 1.940 & 64.77 & 2.99 & 3.00 & 3.00 \\
\hline TFExpa & 0.02 & 99.98 & 1.929 & 64.46 & 2.99 & 2.99 & 3.00 & 0.02 & 99.98 & 1.942 & 64.92 & 2.99 & 2.99 & 2.99 \\
\hline TFExpb & 0.13 & 99.87 & 1.924 & 64.13 & 2.99 & 3.00 & 3.01 & 0.13 & 99.87 & 1.938 & 64.60 & 2.99 & 3.00 & 3.01 \\
\hline TFExpc & 0.53 & 99.47 & 1.932 & 64.31 & 2.98 & 3.00 & 3.02 & 0.54 & 99.46 & 1.945 & 64.77 & 2.98 & 3.00 & 3.02 \\
\hline TFExpd & 0.02 & 99.98 & 1.927 & 64.31 & 2.99 & 3.00 & 3.00 & 0.01 & 99.99 & 1.945 & 64.77 & 2.99 & 3.00 & 3.00 \\
\hline
\end{tabular}

$T F=$ =stimation made with temperate forest data, $B D=$ best fitted distribution for measurement errors, $B D C=$ best fitted distribution by class, Exp=students, a=uDBH, $\mathrm{b}=u T H, \mathrm{c}=u A M, \mathrm{~d}=u$ Plot .

Table IV-17. Error propagation by uncertainty source in AGB estimation, using three scenarios of non-sampling uncertainty in the temperate forests of Durango, Mexico.

Two sources of uncertainty that did not vary between scenarios ( $u$ model and $u$ plot design) had similar contributions to $u W$. The $u$ about the model contributed to a range of 0.52 to $0.54 \%$, while the $u$ of plot design contributed 0.01 to $0.02 \%$.

Among the sources of measurement uncertainty, the lowest contributor was the $u D B H$, with contributions $<0.02 \%$ of the $u W$. Meanwhile, the $u T H$ contribution had a wider range, from 0.01 to $0.13 \%$ of the $u W$.

With the uncertainty estimated at the substratum level, the mean estimated uncertainties by source were compared separately for each scenario, and the paired T-Test was used to compare the mean uncertainty between every pair of sources. With the $M N F I_{0409}$ data, the paired T-Test in the $B D$ scenario indicated that the uncertainty of $u T H-u P l o t$ was not significantly different (pvalue $\left._{\mathrm{tt}}=0.1346\right)$. From the $B D C$ scenario, the sources $u D B H-u T H, u D B H-u P l o t$, and $u T H-u P l o t$ were not significantly different (p-value $\mathrm{t}_{\mathrm{t}} \geq 0.118$ ). In the $\operatorname{Exp}$ scenario, no significant difference was found in the $u D B H-u P l o t$ comparison (p-value $t_{t t} \geq 0.118$ ). 
In the second period of $M N F I_{0914}$, similar results were obtained in the pair comparisons of the $B D C$ and Exp scenarios. For the BDC scenario, a significant difference for the $u D B H-u T H, u D B H-u P l o t$, and $u T H-u P l o t$ comparisons was not found (p-value $\geq 0.1706$ ). For the Exp scenario, the comparison between $u D B H-u$ Plot was not significantly different (p-value $\left.{ }_{t t}=0.2642\right)$. Furthermore, in the $B D$ scenario, all comparisons were statistically different ( $\mathrm{p}$-value $\left.\mathrm{tt}_{\mathrm{t}} \leq 0.0093\right)$. 


\section{IV.4 Empirical study 4: Modelling and mapping AGB for the state of Durango}

\section{IV.4.1 Vegetation in the State of Durango}

The total forested area in the State of Durango (Figure II-5, Section II.2.3) was calculated as $8,509,347$ ha from Series IV and 8,589,123 ha from Series V. The area excluded from the analysis was the area outside of temperate forest, such as areas with predominant anthropogenic activities (i.e. agriculture, pastures, human settlements, etc.), water bodies, and areas with clouds or cloud shadows blocking temperate forest information.

Table IV-18 reports the area occupied by the main vegetation types and land-uses, with emphasis on the temperate forest strata (Figure II-6, Section II.2.3). Comparing the two time-series from 2007 to 2013 , it is noticed an increase in surface area for temperate forest and arid zone vegetation. In contrast, the area with tropical forest and anthropogenic activities decreased. Within the temperate forest, the stratum of the oak forest increased, while the strata of conifer forest and mixed forest decreased.

\begin{tabular}{lrrrr}
\hline \multirow{2}{*}{\multicolumn{1}{c}{ Vegetation and land-use }} & \multicolumn{2}{c}{ Series IV (2007-2008) } & \multicolumn{2}{c}{ Series V (2012-2013) } \\
\cline { 2 - 5 } & \multicolumn{1}{c}{ ha } & \multicolumn{1}{c}{$\%$} & \multicolumn{1}{c}{ ha } & \% \\
\hline Anthropogenic \& no vegetation & $\mathbf{3 8 2 0 1 3 3}$ & $\mathbf{3 0 . 9 8}$ & $\mathbf{3 7 4 0 3 5 7}$ & $\mathbf{3 0 . 3 4}$ \\
Rain forest & $\mathbf{6 0 7 1 8 0}$ & $\mathbf{4 . 9 2}$ & $\mathbf{6 0 5 6 0 7}$ & $\mathbf{4 . 9 1}$ \\
Xeric shrubland & $\mathbf{2 7 3 7 9 4 9}$ & $\mathbf{2 2 . 2 1}$ & $\mathbf{2 8 1 7 4 5 3}$ & $\mathbf{2 2 . 8 5}$ \\
Temperate forest & $\mathbf{5 1 6 4 2 1 8}$ & $\mathbf{4 1 . 8 9}$ & $\mathbf{5 1 6 6 0 6 3}$ & $\mathbf{4 1 . 9 0}$ \\
\multicolumn{1}{r}{ Stratum of conifer forest } & 2359391 & 19.14 & 2356893 & 19.12 \\
Stratum of mixed forest & 1907236 & 15.47 & 190311 & 15.43 \\
Stratum of oak forest & 897591 & 7.28 & 906054 & 7.35 \\
\hline Total & $\mathbf{1 2 3 2 9 4 8 0}$ & & $\mathbf{1 2 3 2 9 4 8 0}$ & \\
\hline
\end{tabular}

Table IV-18. Surface area by vegetation type and land-use for two time-series in the state of Durango, Mexico.

As described in Chapter III, the vegetation series IV and V were used to define the areas and vegetation types in the MNFI (CONAFOR, 2012c, 2017a).

\section{IV.4.2 Basal area and AGB estimation models}

The estimates of basal area $(G)$ and AGB $(W)$ at the cluster level, analyzed in the previous chapter, were the response variables. Therefore, it was this information that was used to adjust the $G$ and $W$ models with the predictor variables derived from Landsat images as response variables for the two periods of study.

\section{IV.4.2.1 Pre-selected predictor variables}

Prior to the adjustment of the models, the pre-selection of prediction variables $(P V)$ through the Pearson correlation coefficient was made. The correlation was calculated among the $P V$ and were removed the $P V$ with more than $\rho=|0.97|$, to avoid multicollinearity, preserving the $P V$ with higher correlation with the response variable (Dohoo et al., 1996). Table IV-19 shows the PVs selected, where is shown the Pearson coefficient and the significance p-value $\leq 0.001$ of the $38 P V$ for the 2007 dataset and $41 P V$ for the 2013 dataset. 
The correlation coefficients ranged from $|0.40|$ to $|0.79|$, out of which the ARVI, NDVI, SR, SRG, and WDRVI predictor variables had correlations greater than $|0.71|$. Out of the available spectral bands, the NIR band was not selected due to low a correlation with the response variables. The texture with the highest correlation was the weighted mean $(M N)$, followed by the Haralicks correlation $(H C)$. Two other textures, homogeneity $(H O)$ and variance $(V A)$ were selected in a smaller proportion to the previous ones.

\begin{tabular}{|c|c|c|c|c|c|c|c|c|c|}
\hline \multirow{2}{*}{$P V$} & \multicolumn{2}{|c|}{ MNFI (2004-2009) } & \multicolumn{2}{|c|}{ MNFI (2009-2014) } & \multirow{2}{*}{$P V$} & \multicolumn{2}{|c|}{ MNFI (2004-2009) } & \multicolumn{2}{|c|}{ MNFI (2009-2014) } \\
\hline & $G$ & $W$ & $G$ & $W$ & & $G$ & $W$ & $G$ & $W$ \\
\hline$\overline{C 3}$ & & & $-0.62 * * *$ & $-0.60 * * *$ & $R d 11 M N$ & -0.61 & -0.59 *** & $-0.60 * * *$ & $-0.58 * * *$ \\
\hline$B 3$ & $-0.71 * * *$ & $-0.64 * * *$ & $-0.63 * * *$ & $-0.62 * * *$ & ND3HC & & & $0.56 * * *$ & $0.58 * * *$ \\
\hline$G 3$ & $-0.70 * * *$ & $-0.63 * * *$ & $-0.61 * * *$ & $-0.60 * * *$ & $N D 3 M N$ & $0.64 * * *$ & $0.66 * * *$ & $0.66 * * *$ & $0.67 * * *$ \\
\hline$R d 3$ & $-0.70 * * *$ & $-0.63 * * *$ & $-0.65 * * *$ & $-0.63 * * *$ & ND7HC & & & $0.54 * * *$ & $0.56 * * *$ \\
\hline SWIR13 & $-0.66 * * *$ & $-0.59 * * *$ & $-0.63 * * *$ & $0.62 * * *$ & $N D 7 M N$ & $0.65 * * *$ & $0.67 * * *$ & $0.66 * * *$ & $0.67 * * *$ \\
\hline SWIR23 & $-0.71 * * *$ & $-0.64 * * *$ & $-0.67 * * *$ & $-0.66 * * *$ & $\mathrm{ND} 11 \mathrm{HC}$ & & & $0.56 * * *$ & $0.57 * * *$ \\
\hline$S R 3$ & $0.78 * * *$ & $0.74 * * *$ & $0.72 * * *$ & $0.76 * * *$ & $N D 11 M N$ & $0.66 * * *$ & $0.67 * * *$ & $0.66 * * *$ & $0.66 * * *$ \\
\hline SRG3 & $0.79 * * *$ & $0.74 * * *$ & $0.71 * * *$ & $0.75 * * *$ & $W D R 3 H C$ & & & $0.52 * * *$ & $0.58 * * *$ \\
\hline ND3 & $0.79 * * *$ & $0.73 * * *$ & $0.75 * * *$ & $0.76 * * *$ & $W D R 3 M N$ & $0.64 * * *$ & $0.67 * * *$ & $0.70 * * *$ & $0.73 * * *$ \\
\hline E3 & $0.73 * * *$ & $0.66 * * *$ & $0.69 * * *$ & $0.72 * * *$ & WDR7HO & & & $-0.50 * * *$ & $0.51 * * *$ \\
\hline$A R 3$ & $0.77 * * *$ & $0.72 * * *$ & $0.75 * * *$ & $0.76 * * *$ & $W D R 7 H C$ & $0.40 * * *$ & $0.45 * * *$ & $0.55 * * *$ & $0.61 * * *$ \\
\hline$S A 3$ & $0.74 * * *$ & $0.69 * * *$ & $0.70 * * *$ & $0.73 * * *$ & $W D R 7 M N$ & $0.67 * * *$ & $0.69 * * *$ & $0.72 * * *$ & $0.74 * * *$ \\
\hline$M S A 3$ & $0.71 * * *$ & $0.67 * * *$ & $0.69 * * *$ & $0.72 * * *$ & WDR11HO & & & $-0.53 * * *$ & $-0.54 * * *$ \\
\hline WDR3 & $0.79 * * *$ & $0.74 * * *$ & $0.74 * * *$ & $0.77 * * *$ & WDR11HC & & & $0.57 * * *$ & $0.63 * * *$ \\
\hline NDMI3 & $0.72 * * *$ & $0.67 * * *$ & $0.72 * * *$ & $0.75 * * *$ & WDR11MN & $0.66 * * *$ & $0.69 * * *$ & $0.71 * * *$ & $0.73 * * *$ \\
\hline$B 3 M N$ & $-0.53 * * *$ & $-0.52 * * *$ & & & WDR11VA & $0.66 * * *$ & $0.69 * * *$ & & \\
\hline$B 7 M N$ & $-0.54 * * *$ & $-0.53^{* * *}$ & $-0.58 * * *$ & $-0.57^{* * *}$ & $A R 3 H C$ & $0.48 * * *$ & $0.51 * * *$ & & \\
\hline$B 11 M N$ & $-0.54 * * *$ & $-0.53 * * *$ & $-0.58 * * *$ & $-0.56^{* * *}$ & $A R 3 M N$ & $0.67 * * *$ & $0.68 * * *$ & $0.71 * * *$ & $0.72 * * *$ \\
\hline$G 3 M N$ & $-0.59 * * *$ & $-0.58 * * *$ & $-0.55^{* * *}$ & $0.53^{* * *}$ & $A R 7 M N$ & $0.69 * * *$ & $0.70 * * *$ & $0.72 * * *$ & $0.73 * * *$ \\
\hline$G 7 M N$ & $-0.59 * * *$ & $-0.56^{* * *}$ & $-0.56^{* * *}$ & $-0.54 * * *$ & $A R 11 M N$ & $0.68 * * *$ & $0.69 * * *$ & $0.72 * * *$ & $0.72 * * *$ \\
\hline$G 11 M N$ & $-0.57^{* * *}$ & $-0.58^{* * *}$ & $-0.56^{* * *}$ & $0.54 * * *$ & $S R G 3 M N$ & $0.62 * * *$ & $0.65 * * *$ & & \\
\hline$R d 3 M N$ & $-0.59 * * *$ & $-0.58 * * *$ & $-0.59 * * *$ & $-0.57 * * *$ & $S R G 3 H C$ & & & $0.66 * * *$ & $0.69 * * *$ \\
\hline$R d 7 M N$ & $-0.61 * * *$ & $-0.59^{* * *}$ & $-0.60 * * *$ & $-0.58^{* * *}$ & $S R G 7 M N$ & $0.64 * * *$ & $0.67 * * *$ & & \\
\hline Rd11HC & $-0.62 * * *$ & $-0.43^{* * *}$ & $-0.60 * * *$ & $-0.41 * * *$ & SRG11MN & $0.63 * * *$ & $0.66 * * *$ & & \\
\hline
\end{tabular}

Table IV-19. Predictor variables $(P V)$ selected to estimate the response variables basal area $(G)$ and AGB $(W)$ in Durango. The description of $P V$ is in Figure III-18.

\section{IV.4.2.2 Estimation models adjusted with MNFI 2004-2009 field data}

The adjusted models for the basal area $(G)$ and AGB $(W)$ estimated from the 2004-2009 MNFI data and Landsat 5 (2007) imagery are given in Table IV-20. The elements contained in the table include vegetation type, response variable, estimator (est), standard error of the estimator ( $S E$ ), the significance of the response variable $(\mathrm{p}=\mathrm{p}>|\mathrm{t}|)$, and the coefficient of determination of model validation $\left(R^{2}\right)$. For each response variable, two models were fitted: the first with the predictor variable $N D V I$ and the second with the variables selected by stepwise regression $(\mathrm{SW})$. The coefficients $(\lambda)$ from the box-cox transformation of the predictor variables were recorded at the bottom of the table. The acronyms of the response variables can be easily followed with the information of Figure III-18 in Chapter III. The goodness-of-fit of the models is described in a further Section.

In the upper part of the table (bold rows) are the models adjusted with the information on the 1662 clusters of the temperate forest $(T F)$. The rest of the table shows the adjusted models for the strata conifer forest $(S C F)$, mixed forest $(S M F)$, and oak forest $(S O F)$, with 292, 1033, and 337 clusters, respectively. 
Using $T F$ models as a reference, it was observed that, when adjusting models for the response variable per stratum, the model of basal area $(G)$ for $S C F$ increased $R^{2}(>0.585)$. On the other hand, the $R^{2}$ decreased for $S M F$ and SOF. W models showed the same $R^{2}$ behavior at the stratum level.

\begin{tabular}{|c|c|c|c|c|c|c|c|c|c|c|}
\hline & \multicolumn{2}{|c|}{ NDVI model } & \multicolumn{3}{|c|}{ Stepwise model } & \multicolumn{2}{|c|}{ NDVI model } & \multicolumn{3}{|c|}{ Stepwise model } \\
\hline \multicolumn{6}{|c|}{ Basal area $(G)$ Temperate forest $(T F)$} & \multicolumn{5}{|c|}{ AGB $(W)$ Temperate forest $(T F)$} \\
\hline $\boldsymbol{R} \boldsymbol{V}$ & Int & $N D 3^{\lambda I}$ & Int & $N D 3^{\lambda I}$ & $R d 11 H C^{\lambda 2}$ & Int & $N D 3^{\lambda 3}$ & & & \\
\hline est & -32.34 & 64.87 & 5.49 & 49.15 & -15.73 & -142.74 & 325.14 & & & \\
\hline$S E$ & 0.98 & 1.42 & 4.34 & 2.19 & 1.79 & 4.21 & 6.85 & & & \\
\hline p-value & $<2 \mathbf{E}^{-16}$ & $<2 \mathbf{E}^{-16}$ & 0.21 & $<2 \mathbf{E}^{-16}$ & $<2 \mathbf{E}^{-16}$ & $<2 \mathbf{E}^{-16}$ & $<2 \mathbf{E}^{-16}$ & & & \\
\hline$R^{2}$ & $\mathbf{0 . 5 8 5}$ & & 0.585 & & & 0.601 & & & & \\
\hline \multicolumn{6}{|c|}{ Basal area $(G)$ Stratum Conifer forest $(S C F)$} & \multicolumn{5}{|c|}{ AGB $(W)$ Stratum Conifer forest $(S C F)$} \\
\hline$R V$ & Int & $N D 3^{\lambda 4}$ & Int & $S R 3^{\lambda 5}$ & & Int & $N D 3^{26}$ & Int & $S R 3^{\lambda 7}$ & \\
\hline est & -46.63 & 80.96 & 34.18 & -50.63 & & -215.2 & 398.20 & 192.71 & -291.09 & \\
\hline$S E$ & 2.63 & 3.68 & 1.07 & 2.26 & & 11.8 & 17.80 & 6.63 & 12.89 & \\
\hline$p$-value & $<2 \mathrm{E}^{-16}$ & $<2 \mathrm{E}^{-16}$ & $<2 \mathrm{E}^{-16}$ & $<2 \mathrm{E}^{-16}$ & & $<2 \mathrm{E}^{-16}$ & $<2 \mathrm{E}^{-16}$ & $<2 \mathrm{E}^{-16}$ & $<2 \mathrm{E}^{-16}$ & \\
\hline$R^{2}$ & 0.624 & & 0.636 & & & 0.646 & & 0.646 & & \\
\hline \multicolumn{6}{|c|}{ Basal area $(G)$ Stratum Mixed forest $(S M F)$} & \multicolumn{5}{|c|}{ AGB $(W)$ Stratum Mixed forest $(S M F)$} \\
\hline$R V$ & Int & $N D 3^{28}$ & Int & $N D 3^{\lambda 8}$ & $R d 11 H C^{29}$ & Int & $N D 3^{210}$ & Int & $N D 3^{2.10}$ & $R d 11 H C^{211}$ \\
\hline est & -32.38 & 65.69 & 20.89 & 45.06 & -22.73 & -129.88 & 333.34 & 479.90 & 243.00 & -466.60 \\
\hline$S E$ & 1.37 & 1.94 & 5.49 & 2.84 & 2.25 & 5.31 & 8.98 & 72.90 & 13.10 & 56.10 \\
\hline$p$-value & $<2 \mathrm{E}^{-16}$ & $<2 \mathrm{E}^{-16}$ & $1.5 \mathrm{E}^{-4}$ & $<2 \mathrm{E}^{-16}$ & $<2 \mathrm{E}^{-16}$ & $<2 \mathrm{E}^{-16}$ & $<2 \mathrm{E}^{-16}$ & $7.50 \mathrm{E}^{-11}$ & $<2 \mathrm{E}^{-16}$ & $2.90 \mathrm{E}^{-16}$ \\
\hline$R^{2}$ & 0.570 & & 0.580 & & & 0.600 & & 0.598 & & \\
\hline \multicolumn{6}{|c|}{ Basal area $(G)$ Stratum Oak forest $(S O F)$} & \multicolumn{5}{|c|}{ AGB $(W)$ Stratum Oak forest $(S O F)$} \\
\hline$R V$ & Int & $N D 3^{\lambda 12}$ & Int & $S R 3^{\lambda l 3}$ & & Int & $N D 3^{\lambda / 4}$ & Int & $S R 3^{\lambda 15}$ & \\
\hline est & 28.61 & -13.80 & 16.05 & -27.30 & & 214.94 & -131.55 & 92.41 & -176.86 & \\
\hline$S E$ & 1.94 & 1.27 & 0.743 & 2.331 & & 12.55 & 9.09 & 3.88 & 11.44 & \\
\hline$p$-value & $<2 \mathrm{E}^{-16}$ & $<2 \mathrm{E}^{-16}$ & $<2 \mathrm{E}^{-16}$ & $<2 \mathrm{E}^{-16}$ & & $<2 \mathrm{E}^{-16}$ & $<2 \mathrm{E}^{-16}$ & $7.50 \mathrm{E}^{-11}$ & $<2 \mathrm{E}^{-16}$ & \\
\hline$R^{2}$ & 0.282 & & 0.298 & & & 0.414 & & 0.422 & & \\
\hline
\end{tabular}

Table IV-20. Adjusted models for the basal area $(G)$ and AGB $(W)$, estimated from the 2004-2009 MNFI.

Predictor variables were also selected stepwise for the $W$ models and resulted in a higher calculated $R^{2}$. Predictions of $G$ and $W$ improved in $S C F$ and $S M F$ by adding Haralicks correlation in window $11 X 11$ for the red band $(R d 11 H C)$. The respective response variables of the SCF and SOF strata did not include texture information. These response variables for $S C F$ was the ratio Red band/NIR (SR3), while for SOF it was the ratio Green band/NIR (SRG3).

\section{IV.4.2.3 Estimation models adjusted with 2009-2014 MNFI field data}

As in the first MNFI period, basal area $(G)$ and AGB $(W)$ estimates with the 2009-2014 MNFI information were the response variables $(R V)$ to fit prediction models using Landsat 8 (2013) imagery. The result of the adjusted models is in Table IV-21, using the same configuration and nomenclature previously presented in Table IV-20.

The model was adjusted for 1635 plots in the temperate forest (TF). At strata level, 216, 1156, and 263 plots were used to adjust the models in the conifer forest $(S C F)$, mixed forest $(S M F)$, and oak forest $(S O F)$. Table IV-21 presents two models adjusted for each stratum, as well as for temperate forest. The first model was adjusted with the NDVI index, and the second model was the result of stepwise regression.

Higher $R^{2}$ was calculated in 2013 compared to 2007, apart from $W$ models in SOF. In comparison to the temperate forest $T F$ models, the strata model of $S C F$ increased the $R^{2}$, while the models of $S M F$ 
and $S O F$ decreased the $R^{2}$. The models fitted for both variables $G$ and $W$ are presented in Table IV21.

The predictor variables selected by the stepwise method for both the $G$ and $W$ models were the same but in a different order. To estimate $G$ in $T F$, the model included the ratio of Red band/NIR (SR3), along with the estimation of the weighted mean of the ARVI index for the $11 X 11$ window $(A R I I M N)$. The stepwise estimate of $W$ in $T F$ selected the NDVI variable (ND).

\begin{tabular}{|c|c|c|c|c|c|c|c|c|c|c|c|}
\hline & \multicolumn{2}{|c|}{ NDVI model } & \multicolumn{4}{|c|}{ Stepwise model } & NDVI & odel & \multicolumn{3}{|c|}{ Stepwise model } \\
\hline \multicolumn{7}{|c|}{ Basal area $(G)$ Temperate forest $(T F)$} & \multicolumn{5}{|c|}{ AGB $(W)$ Temperate forest $(S T F)$} \\
\hline $\begin{array}{c}R V \\
e s t \\
S E \\
p \text {-value } \\
R^{2} \\
\end{array}$ & $\begin{array}{l}\text { Int } \\
-32.91 \\
0.907 \\
<2 E^{-16} \\
0.629 \\
\end{array}$ & \begin{tabular}{r|}
$N D 3^{\lambda I}$ \\
66.06 \\
1.323 \\
$<2 \mathrm{E}^{-16}$
\end{tabular} & \begin{tabular}{r}
\multicolumn{1}{|l}{ Int } \\
$\mathbf{6 2 . 5 1}$ \\
5.78 \\
0.21 \\
0.637 \\
\end{tabular} & $\begin{array}{r}S R 3^{\lambda 2} \\
-33.08 \\
2.45 \\
<2 E^{-16}\end{array}$ & $\begin{array}{r}A R I M N^{23} \\
-101.49 \\
20.09 \\
<2 \mathrm{E}^{-16}\end{array}$ & & \begin{tabular}{r}
\multicolumn{1}{l}{ Int } \\
-128.70 \\
3.69 \\
$<2 E^{-16}$ \\
0.633 \\
\end{tabular} & \begin{tabular}{r|}
$N^{\prime} 3^{24}$ \\
315.70 \\
6.28 \\
$<2 E^{-16}$
\end{tabular} & & & \\
\hline \multicolumn{7}{|c|}{ Basal area $(G)$ Stratum Conifer forest $(S C F)$} & \multicolumn{5}{|c|}{ AGB $(W)$ Stratum Conifer forest $(S C F)$} \\
\hline $\begin{array}{c}R V \\
e s t \\
S E \\
p \text {-value } \\
R^{2} \\
\end{array}$ & $\begin{array}{l}\text { Int } \\
-66.08 \\
3.56 \\
<2 \mathrm{E}^{-16} \\
0.681 \\
\end{array}$ & \begin{tabular}{r|}
$N D 3^{\lambda .5}$ \\
100.73 \\
4.68 \\
$<2 \mathrm{E}^{-16}$
\end{tabular} & \begin{tabular}{r}
\multicolumn{1}{l}{ Int } \\
34.42 \\
1.15 \\
$<2 \mathrm{E}^{-16}$ \\
0.681 \\
\end{tabular} & $\begin{array}{r}S R 3^{\lambda 6} \\
-51.82 \\
2.39 \\
<2 \mathrm{E}^{-16}\end{array}$ & & & $\begin{array}{l}\text { Int } \\
-184.50 \\
11.60 \\
<2 \mathrm{E}^{-16} \\
0.660 \\
\end{array}$ & \begin{tabular}{r|}
$N D 3^{\lambda 7}$ \\
366.90 \\
18.60 \\
$<2 \mathrm{E}^{-16}$
\end{tabular} & $\begin{array}{r}\text { Int } \\
319.80 \\
55.00 \\
<2 \mathrm{E}^{-16} \\
0.674 \\
\end{array}$ & $\begin{array}{r}S R 3^{\lambda 8} \\
-343.30 \\
28.00 \\
<2 \mathrm{E}^{-16}\end{array}$ & $\begin{array}{r}N D 3 H C^{29} \\
-41.40 \\
18.30\end{array}$ \\
\hline \multicolumn{7}{|c|}{ Basal area $(G)$ Stratum Mixed forest $(S M F)$} & \multicolumn{5}{|c|}{ AGB $(W)$ Stratum Mixed forest $(S M F)$} \\
\hline $\begin{array}{c}R V \\
e s t \\
S E \\
p \text {-value } \\
R^{2} \\
\end{array}$ & $\begin{array}{l}\text { Int } \\
-27.98 \\
1.07 \\
<2 \mathrm{E}^{-16} \\
0.598 \\
\end{array}$ & \begin{tabular}{r|}
$N D 3^{\lambda 10}$ \\
61.79 \\
1.58 \\
$<2 \mathrm{E}^{-16}$
\end{tabular} & \begin{tabular}{r}
\multicolumn{1}{c}{ Int } \\
101.24 \\
8.58 \\
$<2 \mathrm{E}^{-16}$ \\
0.611 \\
\end{tabular} & $\begin{array}{r}S R 3^{\lambda 11} \\
-30.14 \\
3.04 \\
1.2 \mathrm{E}^{-11}\end{array}$ & $\begin{array}{r}W D R 11 H O^{\lambda 12} \\
10.00 \\
1.46 \\
1.3 \mathrm{E}^{-14}\end{array}$ & $\begin{array}{r}A R I 1 M N^{213} \\
-213.28 \\
27.28\end{array}$ & $\begin{array}{r}\text { Int } \\
-111.57 \\
4.31 \\
<2 \mathrm{E}^{-16} \\
0.619 \\
\end{array}$ & \begin{tabular}{r|}
$N D 3^{\lambda 14}$ \\
312.19 \\
7.60 \\
$<2 \mathrm{E}^{-16}$
\end{tabular} & $\begin{array}{l}\text { Int } \\
-467.20 \\
123.60 \\
1.6 \mathrm{E}^{-4} \\
0.618 \\
\end{array}$ & $\begin{array}{r}S R 3^{\lambda 15} \\
-198.80 \\
19.40 \\
<2 \mathrm{E}^{-16}\end{array}$ & $\begin{array}{r}A R 11 M N^{\lambda 16} \\
404.00 \\
71.10 \\
1.7 \mathrm{E}-8\end{array}$ \\
\hline \multicolumn{7}{|c|}{ Basal area $(G)$ Stratum Oak forest $(S O F)$} & \multicolumn{5}{|c|}{ AGB $(W)$ Stratum Oak forest $(S O F)$} \\
\hline $\begin{array}{c}R V \\
e s t \\
S E \\
p \text {-value } \\
R^{2}\end{array}$ & $\begin{array}{l}\text { Int } \\
62.85 \\
5.52 \\
<2 \mathrm{E}^{-16} \\
0.313 \\
\end{array}$ & \begin{tabular}{r|}
$N D 3^{217}$ \\
-46.45 \\
4.61 \\
$<2 \mathrm{E}^{-16}$
\end{tabular} & $\begin{array}{r}\text { Int } \\
22.63 \\
4.10 \\
8.8 \mathrm{E}^{-8} \\
0.356 \\
\end{array}$ & $\begin{array}{r}E 3^{\lambda 18} \\
-7.70 \\
2.94 \\
9.4 \mathrm{E}^{-3}\end{array}$ & $\begin{array}{r}{ }^{\prime} D R 7 O^{\lambda 19} \\
7.35 \\
1.81 \\
6.8 \mathrm{E}^{-5}\end{array}$ & $\begin{array}{r}W D R 7 M N^{\lambda 20} \\
-11.01 \\
1.69 \\
4.4 \mathrm{E}^{-10}\end{array}$ & $\begin{array}{l}\text { Int } \\
774.30 \\
65.30 \\
<2 \mathrm{E}^{-16} \\
0.372 \\
\end{array}$ & \begin{tabular}{r|}
$N D 3^{\lambda 21}$ \\
-687.50 \\
60.50 \\
$<2 \mathrm{E}^{-16}$
\end{tabular} & \begin{tabular}{l}
\multicolumn{1}{l}{ Int } \\
807.07 \\
77.56 \\
$<2 \mathrm{E}^{-16}$ \\
0.388
\end{tabular} & $\begin{array}{r}\text { SWIR2_3.22 } \\
-0.08 \\
0.02 \\
9.1 \mathrm{E}^{-5}\end{array}$ & $\begin{array}{r}M S A 3^{\lambda 23} \\
-670.01 \\
71.66 \\
<2 \mathrm{E}^{-16}\end{array}$ \\
\hline
\end{tabular}

Int=intercept, $\lambda=$ Box-Cox coefficient, $G=$ Basal area $\left(\mathrm{m}^{2} \mathrm{ha}^{-1}\right), W=$ Aboveground biomass $\left(\mathrm{Mg}^{-1}\right), \lambda 1=0.383838=0 . \widehat{38}, \lambda 2=-0 . \widehat{94}, \lambda 3=-0 . \widehat{42}, \lambda 4=0 . \widehat{54}$,

$\lambda 5=0 . \widehat{26}, \lambda 6=-1 . \widehat{03}, \lambda 7=0 . \widehat{46}, \lambda 8=-0 . \widehat{82}, \lambda 9=0 . \widehat{10}, \lambda 10=0 . \widehat{42}, \lambda 11=-0 . \widehat{86}, \lambda 12=1 . \widehat{67}, \lambda 13=-0 . \widehat{38}, \lambda 14=0 . \widehat{62}, \lambda 15=-0 . \widehat{66}, \lambda 16=0 . \widehat{18}, \lambda 17=-0 . \widehat{14}, \lambda 18=-0 . \widehat{30}, \lambda 19=2$, $\lambda 20=0 . \widehat{98}, \lambda 21=-0 . \widehat{06}, \lambda 22=0 . \widehat{74}, \lambda 23=-0 . \widehat{06}$

Table IV-21. Adjusted models for the basal area $(G)$ and AGB $(W)$, estimated with the 2009-2014 $M N F I$ data.

The mixed forest stratum $(S M F)$ contained the largest number of clusters. The corresponding stepwise analysis selected the same variables as $T F(S R 3$ and $A R I 1 M N)$, along with the variable $W D R 11 H O$. For the $W$ estimation, the model selected the $S R 3$ and ARIIMN variables.

The model adjusted to estimate $G$ in the conifer forest ( $S C F)$ included the variable $S R 3$, while the model to estimate $W$ added the $N D 3 H C$ variable to better fit the model.

In the oak forest (SOF), the models for $G$ and $W$ used entirely different variables. The model adjusted for $G$ selected E3, WDR7HO, and WDR7MN, and the model adjusted for $W$ included SWIR2 and MSAVI (MSA).

\section{IV.4.2.4 Goodness-of-fit of adjusted models}

For the estimated models, absolute bias and relative bias were calculated (Bias, Biasr) and reported the results in Table IV-22, together with RMSE, CV (RMSE/mean), and $R^{2}$. The top part of the table shows the information of the 2004-2009 MNFI, and the bottom part shows the information of the 2009-2014 MNFI.

The stepwise models had lower RMSE and $C V$ compared to NDVI models adjusted for the same vegetation type and for the same response variables $(G$ or $W)$. For example, the $T F$ model adjusted 
for $N D V I$ had an $R M S E=4.35 \mathrm{~m}^{2} \mathrm{ha}^{-1}$ and $C V=34.36 \%$, while the stepwise model reported an $R M S E=4.28 \mathrm{~m}^{2} \mathrm{ha}^{-1}$ and $C V=33.78 \%$. The Bias was negative for all estimates, indicating an underestimation of the fitted models. However, two optimized models had higher Bias (more negative) than was calculated in the NDVI models: $G$ estimation in $T F$ and $W$ estimation in $S M F$, both of which were derived from the 2004-2009 MNFI.

\begin{tabular}{|c|c|c|c|c|c|c|c|c|c|c|}
\hline \multirow{2}{*}{$\begin{array}{c}\text { Model } \\
\text { MNFI 2004-2009 }\end{array}$} & \multicolumn{5}{|c|}{ Basal area $(G)$} & \multicolumn{5}{|c|}{$\operatorname{AGB}(W)$} \\
\hline & $R^{2}$ & $\begin{array}{l}\text { RMSE } \\
\mathrm{m}^{2} \mathrm{ha}^{-1}\end{array}$ & $\begin{array}{l}C V \\
\%\end{array}$ & $\begin{array}{c}\text { Bias } \\
\mathrm{m}^{2} \mathrm{ha}^{-1}\end{array}$ & $\begin{array}{c}\text { Bias } r \\
\%\end{array}$ & $R^{2}$ & $\begin{array}{c}R M S E \\
\mathrm{Mg} \mathrm{ha}^{-1}\end{array}$ & $\begin{array}{l}C V \\
\%\end{array}$ & $\begin{array}{c}\text { Bias } \\
\mathrm{Mg} \mathrm{ha}^{-1}\end{array}$ & $\begin{array}{c}\text { Bias } r \\
\%\end{array}$ \\
\hline$T F(N D V I)$ & $\underline{0.585}$ & 4.35 & 34.36 & -0.28 & -2.25 & $\underline{0.601}$ & $\underline{24.50}$ & 40.48 & -3.37 & -5.57 \\
\hline$T \overline{F \text { (stepwise) }}$ & $\overline{0.585}$ & $\overline{4.28}$ & $\overline{33.78}$ & $\underline{-0.29}$ & $\underline{-2.32}$ & & & & & \\
\hline$\overline{S C F(N D V I)}$ & $\overline{0.624}$ & $\overline{4.38}$ & $\overline{38.08}$ & $\overline{-0.36}$ & $\overline{-3.09}$ & 0.646 & 24.37 & 47.21 & -3.91 & -7.58 \\
\hline$S C F$ (stepwise) & 0.636 & 4.33 & 37.62 & -0.28 & -2.42 & 0.646 & 24.21 & 46.90 & -3.59 & -6.95 \\
\hline$S M F(N D V I)$ & 0.570 & 4.35 & 30.11 & -0.41 & -2.83 & 0.600 & 25.50 & 36.04 & -3.53 & -4.99 \\
\hline SMF (stepwise) & 0.580 & 4.23 & 29.28 & -0.38 & -2.65 & 0.598 & 24.71 & 34.92 & -3.98 & -5.62 \\
\hline$S O F(N D V I)$ & 0.282 & 3.39 & 41.61 & -0.42 & -5.16 & 0.414 & 17.35 & 47.48 & -1.87 & -5.13 \\
\hline SOF (stepwise) & 0.298 & 3.33 & 40.73 & -0.39 & -4.83 & 0.422 & 17.05 & 46.67 & -1.41 & -3.87 \\
\hline \multicolumn{11}{|l|}{ MNFI 2009-2014 } \\
\hline$\underline{\mathrm{TF}}(N D V I)$ & 0.629 & $\underline{4.18}$ & 32.53 & -0.46 & -3.62 & $\underline{0.633}$ & 23.93 & 39.65 & -3.52 & -5.84 \\
\hline$\underline{T F \text { (stepwise) }}$ & $\overline{0.637}$ & $\underline{4.11}$ & $\overline{32.01}$ & $\underline{-0.39}$ & -3.02 & & & & & \\
\hline$S C F(N D V I)$ & $\overline{0.681}$ & $\overline{4.26}$ & 39.27 & -0.35 & -3.25 & 0.660 & 23.88 & 51.71 & -3.61 & -7.82 \\
\hline$S C F$ (stepwise) & 0.681 & 4.24 & 39.02 & -0.35 & -3.21 & 0.674 & 23.20 & 50.25 & -3.28 & -7.11 \\
\hline$S M F(N D V I)$ & 0.598 & 4.31 & 29.99 & -0.50 & -3.50 & 0.619 & 25.22 & 36.75 & -3.67 & -5.34 \\
\hline SMF (stepwise) & 0.611 & 4.19 & 29.23 & -0.43 & -3.00 & 0.618 & 25.18 & 36.69 & -3.26 & -4.78 \\
\hline$S O F(N D V I)$ & 0.313 & 3.26 & 41.85 & -0.38 & -4.91 & 0.372 & 16.76 & 47.87 & -2.27 & -6.49 \\
\hline SOF (stepwise) & 0.356 & 3.06 & 39.26 & -0.33 & -4.30 & 0.388 & 16.06 & 45.87 & -1.77 & -5.07 \\
\hline
\end{tabular}

Table IV-22. Bias estimate and goodness-of-fit of the adjusted models for the basal area $(G)$ and AGB $(W)$ estimated from MNFI data in Durango, Mexico.

\section{IV.4.2.4.1 Stepwise regression models by strata for MNFI 2004-2009}

The indicators of basal area $(G)$ and AGB $(W)$ in temperate forest $(T F)$ models, using information from the first MNFI measurement in Durango, are displayed in the underlined rows.

By separating the $T F$ area in the strata and fitting models for each stratum, some fitting parameters changed. In the $G$ estimation for $S C F$, the $R^{2}$ increased (0.636) and the Bias decreased $\left(-0.28 \mathrm{~m}^{2}\right.$ ha $\left.{ }^{1}\right)$. In the $S M F$ model, the RMSE and $C V$ indicators were lower $\left(4.23 \mathrm{~m}^{2} \mathrm{ha}^{-1}\right.$ and $\left.29.28 \%\right)$ than the estimates in $T F$. Conversely, in the SOF model, the prediction of the model decreased even though the RMSE was lower $\left(3.33 \mathrm{~m}^{2} \mathrm{ha}^{-1}\right)$ than the estimated in the model for $T F$.

With respect to the $W$ estimate in the SCF model, $R^{2}$ (0.646) increased and RMSE (24.21 $\left.\mathrm{Mg} \mathrm{ha}^{-1}\right)$ was reduced. In the $S M F$ model, $C V=34.92 \%$ was reduced. The adjusted model for $S O F$ had a lower $R^{2}(0.422)$, but nevertheless reduced the variance $\left(R M S E=17.05 \mathrm{Mg} \mathrm{ha}^{-1}\right)$ and the Bias $(-1.414 \mathrm{Mg}$ $\left.\mathrm{ha}^{-1}\right)$.

\section{IV.4.2.4.2 Stepwise regression models by strata for MNFI 2009-2014}

In the second period of MNFI, indicators of the $G$ model suggest a better prediction compared to the first MNFI model for $T F$ predictions. The differences consisted of increasing $R^{2}=0.637$ and decreasing both $R M S E=4.11 \mathrm{~m}^{2} \mathrm{ha}^{-1}$ and $C V=32.01 \%$.

The stratum model indicators improved when compared to the $T F$-adjusted model. For example, the $S C F$ model had an increase of $R^{2}(0.681)$ and a decrease of Bias $\left(-0.35 \mathrm{~m}^{2} \mathrm{ha}^{-1}\right)$. In the SMF model, 
$C V(29.23 \%)$ and relative Bias $(-3.00 \%)$ decreased. For the SOF model, RMSE $\left(3.06 \mathrm{~m}^{2} \mathrm{ha}^{-1}\right)$ and Bias $\left(-0.33 \mathrm{~m}^{2} \mathrm{ha}^{-1}\right)$ decreased.

The $W$ estimate for $T F$ had changes in the indicators compared to the first period. the observed increase in $R^{2}(0.633)$ and decrease in $C V(39.65 \%)$ and Bias $\left(-3.52 \mathrm{Mg} \mathrm{ha}^{-1}\right)$ suggested an improvement of the model.

As in $G$ models, $W$ models improved goodness-of-fit of the models adjusted in $T F$. The $S C F$ model had larger $R^{2}(0.674)$, smaller RMSE (23.20 $\left.\mathrm{Mg} \mathrm{ha}^{-1}\right)$, and smaller Bias $\left(\mathrm{Mg} \mathrm{ha}^{-1}\right)$. In the SMF model decreased $C V(36.69 \%)$ and decreased Bias $\left(-3.26 \mathrm{Mg} \mathrm{ha}^{-1}\right)$. Finally, the SOF model calculated a lower $R M S E=16.06 \mathrm{Mg} \mathrm{ha}^{-1}$ and a lower Bias $=-1.77 \mathrm{Mg} \mathrm{ha}^{-1}$.

\section{IV.4.3 Basal area and AGB in the estimation}

Using the stepwise regression models and applying the results to the Landsat imagery, the basal area $(G)$ and AGB $(W)$ were estimated in the temperate forest of Durango, Mexico. Then, the statistics (mean and standard deviation) for the variable estimates of the temperate forest $(T F)$ and per-vegetation stratum were calculated (Table IV-23). In this table, the total area where Landsat imagery information was properly acquired and applied to the models was included (non-temperate forests and clouds were excluded). In the bold character rows, the results of applying the stepwise model in $T F$ ( $\mathrm{n}=1662$ in 2007 and $\mathrm{n}=1635$ in 2013) are shown for both response variables. For the $W$ estimate, the calculation of total storage was included (Tg). Similarly, $G$ and $W$ statistics for each vegetation stratum were estimated $(S C F, S M F$, and $S O F)$. With the stratum estimates, a single layer of temperate forest merged was made (TFM).

\begin{tabular}{|c|c|c|c|c|c|c|c|c|}
\hline \multirow{2}{*}{ Time } & \multirow{2}{*}{ Area } & \multicolumn{3}{|c|}{ Basal area $(G)$} & \multicolumn{4}{|c|}{ Aboveground biomass $(W)$} \\
\hline & & $\hat{\mu}$ & $\hat{\sigma}$ & $R M S E$ & $\hat{\mu}$ & $\hat{\sigma}$ & $R M S E$ & storage \\
\hline 2007 & ha & \multicolumn{3}{|c|}{$\mathrm{m}^{2} \mathrm{ha}^{-1}$} & \multicolumn{3}{|c|}{$\mathrm{Mg} \mathrm{ha}^{-1}$} & $\mathrm{Tg}$ \\
\hline$T F(n=1662)$ & 52172.76 & 12.58 & 5.33 & 4.28 & 58.56 & 31.43 & 24.50 & 301.70 \\
\hline $\operatorname{TFM}(n=1662)$ & .76 & 12.16 & 5.53 & - - & 55.36 & 31.28 & -- & 285.25 \\
\hline$S C$ & 8 & 13.72 & 5.59 & 4.33 & 62.11 & 31.27 & 24.21 & 146.34 \\
\hline 033) & 5 & 12.32 & 5.41 & 23 & 56.57 & 32.73 & 24.71 & 107.86 \\
\hline$F(n=337$ & & 7.70 & 2.35 & 33 & 34.90 & 15.38 & 17.05 & 31.04 \\
\hline \multicolumn{9}{|l|}{2013} \\
\hline$T F(n=1635)$ & 5104018.71 & 12.42 & 5.61 & 4.11 & 57.03 & 32.51 & 23.93 & 291.09 \\
\hline$T F M(n=1635)$ & 5104018.71 & 11.99 & 5.83 & - - & 53.57 & 32.01 & - & 273.42 \\
\hline$\Gamma$ & & 14.03 & $6.1 \mathrm{c}$ & 24 & 61.64 & & 23.20 & 144.15 \\
\hline$F(n=$ & & 11.65 & 5.12 & 4.19 & 52.18 & 31.59 & 25.18 & 98.31 \\
\hline $\operatorname{SOF}(n=263)$ & 880943.85 & 7.33 & 2.68 & 3.06 & 35.10 & 14.88 & 16.06 & 30.92 \\
\hline
\end{tabular}

Table IV-23. Basal area $(G)$ and AGB $(W)$, estimate for temperate forest in Durango, Mexico.

Basal area models estimated values less than zero for $0.17 \%$ of the area in 2007 and $0.27 \%$ of the area in 2013. Moreover, from applying the AGB models, the area with values less than zero was $1.90 \%$ in 2007 and $1.78 \%$ in 2013 . Values less than zero were estimated because the range of predictor variables used $(P V)$ to fit the regression models has not covered all the range of $P V$ values in the study area, previously referred to as gaps (Section I.2.2.2.2). In this study, it was the abovementioned percentage of area for basal area and AGB. These values less than zero were replaced by 
$0.1\left(\mathrm{~m}^{2} \mathrm{ha}^{-1} / \mathrm{Mg} \mathrm{ha}^{-1}\right)$ with no modification to the estimates of $\hat{\mu}$ and $\hat{\sigma}$. Los valores menores a ceros se obtuvieron

\section{IV.4.3.1 Basal area $(G)$ estimates for Durango temperate forest}

In 2007, the $G$ estimate using the $T F$ model $\left(\hat{\mu}=12.58 \mathrm{~m}^{2} \mathrm{ha}^{-1}\right)$ was larger than the TFM estimate $\left(\hat{\mu}=12.16 \mathrm{~m}^{2} \mathrm{ha}^{-1}\right)$. In contrast, $\hat{\sigma}$ was larger in $\operatorname{TFM}\left(5.33 \mathrm{~m}^{2} \mathrm{ha}^{-1}\right)$. Estimates of $G$ for 2013 were like in 2007, with a larger $\hat{\mu}$ in $T F\left(12.42 \mathrm{~m}^{2} \mathrm{ha}^{-1}\right)$ than in $\operatorname{TFM}\left(11.99 \mathrm{~m}^{2} \mathrm{ha}^{-1}\right)$. The $\hat{\sigma}$ in 2013 was smaller in $T F$ than in TFM (5.61 and $\left.5.83 \mathrm{~m}^{2} \mathrm{ha}^{-1}\right)$.

The $\hat{\mu} G$ values across the different vegetation strata were largest for $S M F$ in 2007, with a value of $12.32 \mathrm{~m}^{2} \mathrm{ha}^{-1}$. However, in 2013, the SCF and SOF estimates were the largest (14.03 and $7.33 \mathrm{~m}^{2} \mathrm{ha}^{-}$ ${ }^{1}$ ). Furthermore, the $\hat{\sigma}$ was smaller in 2007 for SCF and SOF (5.59 and $2.35 \mathrm{~m}^{2} \mathrm{ha}^{-1}$ ) but was smaller for $S M F$ in $2013\left(5.12 \mathrm{~m}^{2} \mathrm{ha}^{-1}\right)$.

\section{IV.4.3.2 AGB (W) estimates for the Durango temperate forests}

The $2007 \hat{\mu} W$ estimates were larger in $T F$ compared to $T F M\left(58.56>55.36 \mathrm{Mg} \mathrm{ha}^{-1}\right)$. Therefore, storage was also larger for $T F$ at $301.70 \mathrm{Tg}\left(\mathrm{Tg}=10^{6} \mathrm{Mg}\right)$. The $\hat{\sigma}$ was smaller in $T F M$, with a value of $31.28 \mathrm{Mg} \mathrm{ha}^{-1}$. The 2013 trends were the same, in which $T F$ had a $\hat{\mu}$ of $57.03 \mathrm{Mg} \mathrm{ha}^{-1}, \hat{\sigma}$ of 32.51 $\mathrm{Mg} \mathrm{ha}^{-1}(C V=0.6)$, and storage of $291.09 \mathrm{Tg}$.

For the vegetation strata in 2007, the $\hat{\mu} W$ estimates were larger in the SCF and SMF (62.11 and $56.57 \mathrm{Mg} \mathrm{ha}^{-1}$ ), while the larger SOF estimate occurred in 2013 (35.10 Mg ha-1). The $\hat{\sigma}$ was smaller in 2007 for $S C F$, with $31.27 \mathrm{Mg} \mathrm{ha}^{-1}$. However, for $S O F$ and $S M F$, it was smaller in 2013 (14.88 and $\left.31.59 \mathrm{Mg} \mathrm{ha}^{-1}\right)$.

\section{IV.4.3.3 Basal area and AGB maps of Durango, Mexico}

Using the models per stratum described above, the basal area $(G)$ and AGB $(W)$ maps were produced for the State of Durango. The results, presented in Figure IV-13, show the $G$ estimated by the stepwise regression models using Landsat satellite imagery and the INEGI vegetation series. The values in the figure for the Landsat 5 imagery (2007) range from 0 to $35.14 \mathrm{Mg} \mathrm{ha}^{-1}$ and from 0 to $35.49 \mathrm{Mg} \mathrm{ha}^{-1}$ for the Landsat 8 imagery (2013). Surfaces with the presence of clouds were excluded in the Landsat 8 images (right), located in the far west of the state in two blank areas. This surface spanned 48,154 ha without information in 2013. However, the same land area was able to be analyzed in 2007 and averaged a $15.43 \mathrm{~m}^{2} \mathrm{ha}^{-1}$ basal area.

In $2007,88.6 \%$ of the evaluated area in the temperate forest $(T F)$ registered less than $20 \mathrm{~m}^{2} \mathrm{ha}^{-1}$ of $G$. The class with the largest area was (10 to 15$] \mathrm{m}^{2} \mathrm{ha}^{-1}$, comprising $30.5 \%$ of the $T F$. Moreover, with the information from $2013,87.1 \%$ of the surface recorded less than $20 \mathrm{~m}^{2} \mathrm{ha}^{-1}$. The largest surface class in this year was $\left(5\right.$ to $10 \mathrm{~cm}$ ] $\mathrm{m}^{2} \mathrm{ha}^{-1}$, covering $32.2 \%$ of the $T F$ in Durango (see Table VIII-43, Appendix VIII). 


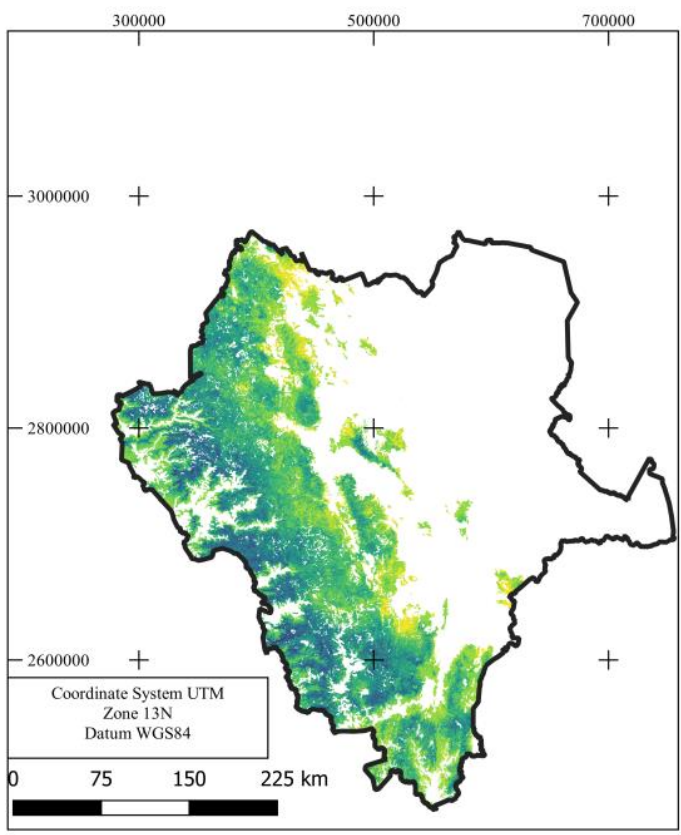

MNFI (2004-2009) and Landsat 5 (2007)

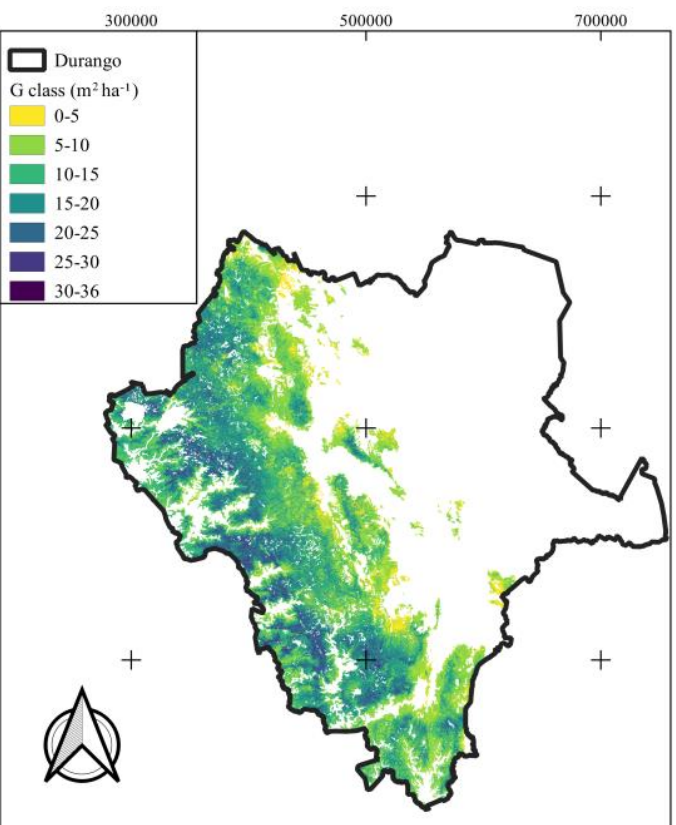

MNFI (2009-2014) and Landsat 8 (2013)

Figure IV-13. Basal area in the temperate forest of Durango, Mexico, with information from the MNFI and Landsat imagery.

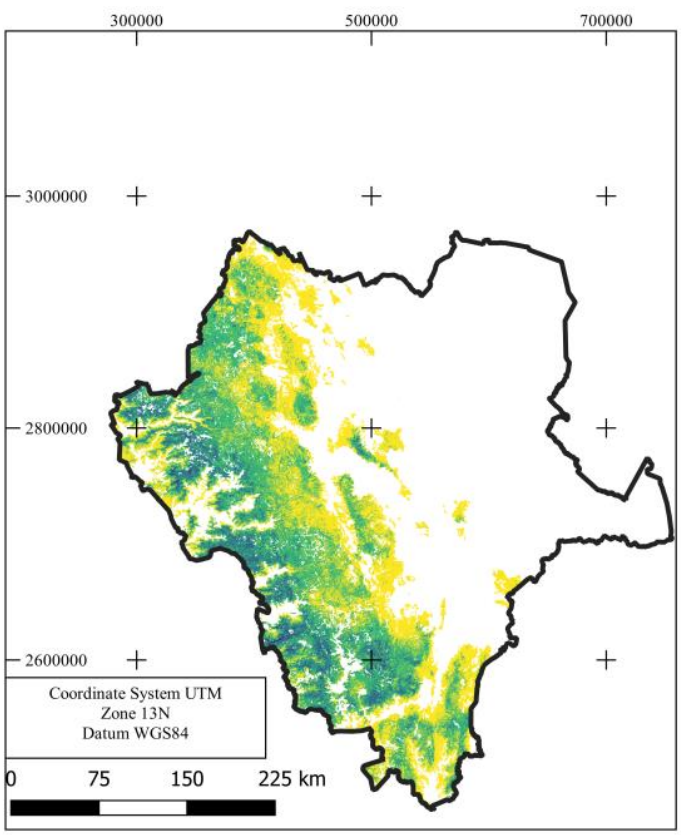

MNFI (2004-2009) and Landsat 5 (2007)

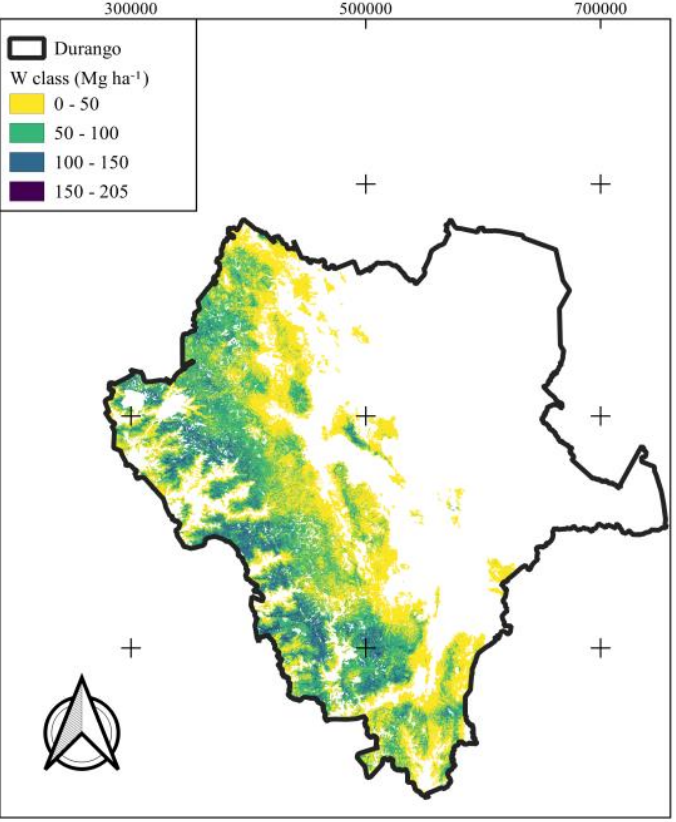

MNFI (2009-2014) and Landsat 8 (2013)

Figure IV-14. AGB in the temperate forest of Durango, Mexico, with information from the MNFI and Landsat imagery.

Figure IV-14 shows the distribution of the $W$ in the temperate forest of Durango. This map was produced by applying the stepwise regression models of $W$ to Landsat imagery. The $W$ range in 2007 was 0 to $199.48 \mathrm{Mg} \mathrm{ha}^{-1}$ and in 2013 was 0 to $201.79 \mathrm{Mg} \mathrm{ha}^{-1}$. According to the 2007 
estimates, $77.5 \%$ of $W$ was contained in classes less than $100 \mathrm{Mg} \mathrm{ha}^{-1}$, which represented $89.9 \%$ of the temperate forest area. Similarly, in 2013, the two classes less than $100 \mathrm{Mg} \mathrm{ha}^{-1}$ covered $89.6 \%$ of the area, with an estimate of $76.03 \%$ of the AGB in TF. Thus, classes $\geq 100 \mathrm{Mgha}^{-1}$ increased surface in 2013 (see Table VIII-44, Appendix VIII). For the area covered by clouds in 2013, a storage of $3.8 \mathrm{Tg}$ in 2007 was calculated.

\section{IV.4.3.4 Comparison of model-based and sampling-based estimations}

The estimates of linear regression models (Table IV-23) were compared with the estimates from the sampling-based method made in the MNFI (Section IV.3 of this study). In this case, the estimators $(\hat{\mu}, \hat{\sigma}, S E, R E)$ calculated for both methods are available in Table IV-24. In this table, the calculation of AGB storage ( $T g$ ) and the uncertainty in estimating AGB storage $(u T g)$ using the $\hat{\mu}$ and $S E$ estimators were included. Calculations are given for the total reported area of the temperate forest $(T F)$ and the per-strata vegetation in Durango to estimate total storage using both estimation methods. Estimates by stratum were summed up and reported as temperate forest merged (TFM).

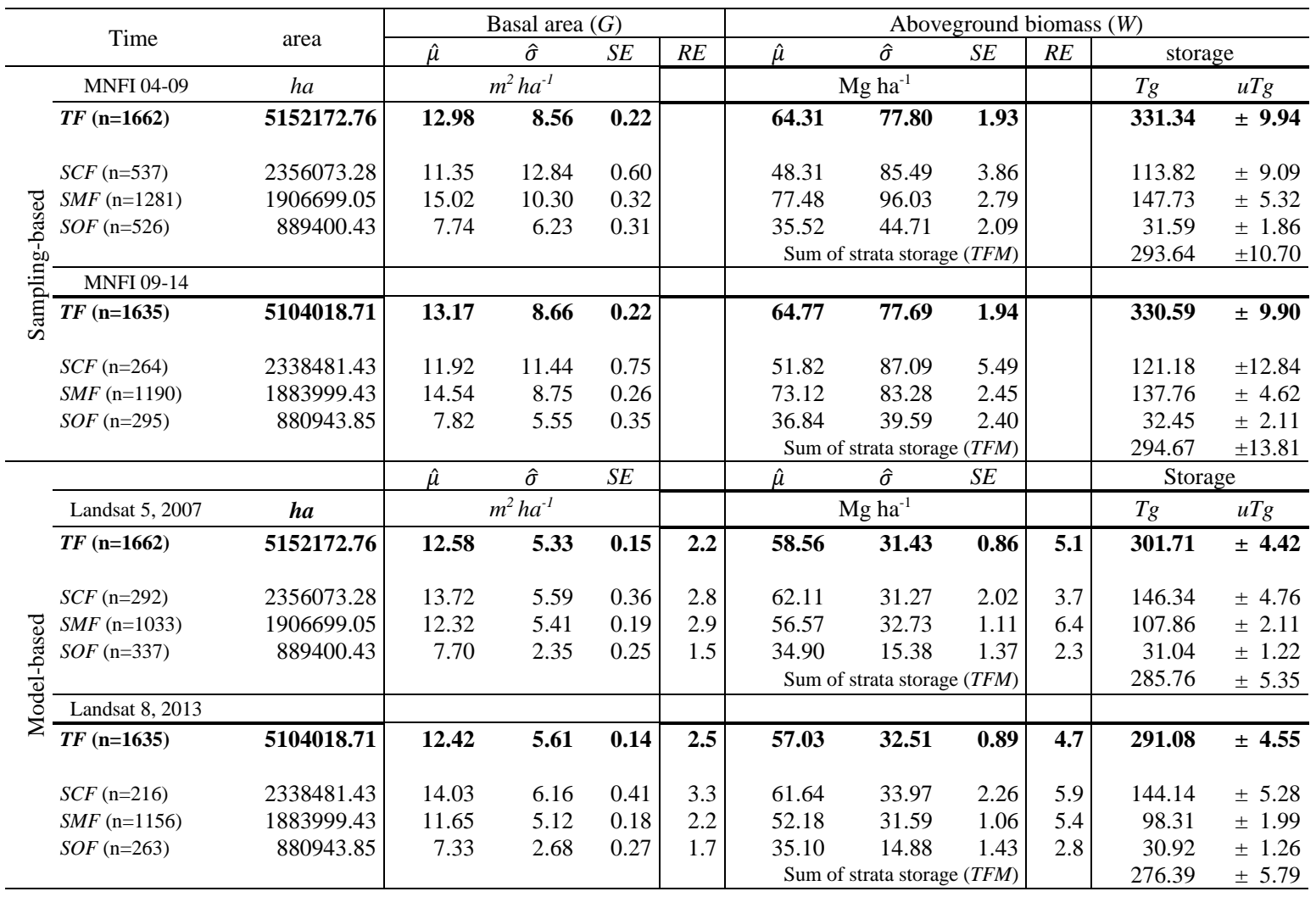

Table IV-24. Estimates of basal area $(G)$ and AGB $(W)$ using methods based on forest inventory sampling and regression models, applied in the temperate forests of Durango, Mexico.

The results from both methods in the temperate forest $(T F)$ showed that in the first study period the sampling-based value of $\hat{\mu}$ was larger than the model-based value (bold rows in Table IV-24). This observation was valid for the two variables and both study periods. It was also found that the sampling-based method produced larger estimates of $\hat{\mu}$ in the second period of the MNFI, while the opposite result was achieved with the model-based method. 
At the stratum level, was observed similar behavior as in $T F$ for most strata. Nevertheless, when the sampling-based method was applied in the mixed forest stratum $(S M F)$, the $\hat{\mu}$ value was smaller in the second period for both variables. On the other hand, the model-based method in $G$ estimation calculated an increase in the estimation in the second period for the conifer forest stratum ( $S C F$, $\left.14.03>13.72 \mathrm{~m}^{2} \mathrm{ha}^{-1}\right)$. This result was also observed in the oak forest stratum (SOF) for $W$ $\left(34.90<35.10 \mathrm{Mg} \mathrm{ha}^{-1}\right)$.

SOF estimates were similar for both methods and for both variables, with ranges of 7.33 to $7.82 \mathrm{~m}^{2}$ ha $^{-1}$ for $G$ and 34.90 to $36.84 \mathrm{Mg} \mathrm{ha}^{-1}$ for $W$. In the other two strata, the dominance alternated according to the estimation method. While $S C F$ had the largest $\hat{\mu}$ in the sampling-based method, $S M F$ had the largest estimate in model-based method.

$W$ storage behaved like the $\hat{\mu}$ estimator. Thus, the largest storage from the sampling-based method came from the $S M F$, with values of $147.73 \mathrm{Tg}$ for the first MNFI and $137.76 \mathrm{Tg}$ for the second MNFI. Meanwhile, the largest storage in the model-based method was in the $S C F$.

Comparing the $W$ stored in the TF (bold rows in Table IV-24) to the sum of $W$ stored by stratum, an overestimation of the $W$ in $T F$ was observed. The sampling-based estimate from all sampled clusters of the $T F$ was $13 \%$ larger than the sum of the estimates per vegetation stratum. Likewise, the overestimate of $W$ in the model-based method was $6 \%$.

In Figure IV-15, the storage values of AGB with information from the two estimation methods were plotted (sampling-based and model-based). This figure included the uncertainty confidence interval ( $C I_{95 \%}-95 \%$ of probability-), using $S E$ for the sampling-based and model-based method, respectively.

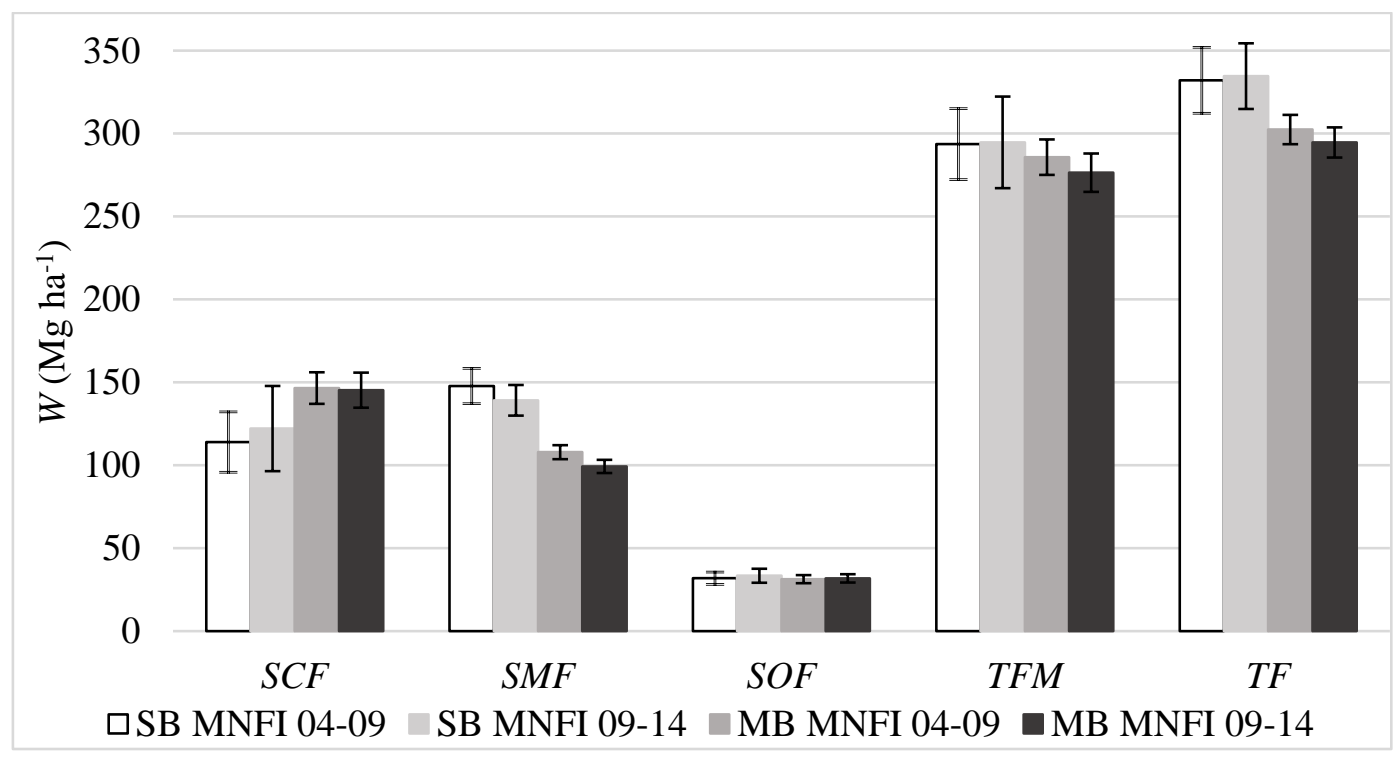

$S B=$ sampling-based method, $M B=$ model-based method

Figure IV-15. AGB storage and uncertainty in AGB storage using information from two methods of estimation in the temperate forests of Durango, Mexico.

Figure IV-15 shows that AGB's estimates in temperate forest $(T F)$ were higher than those observed for temperate forest merged (TFM). However, the confidence intervals of the two methods of AGB 
estimation showed overlap in the TFM estimates; this overlap was not observed in the results for $T F$. This figure shows also the AGB overestimate in the results of the model-based method for the conifer forest stratum $(S C F)$, as well as the underestimate of the same method for the mixed forest stratum $(S M F)$, compared to the sampling-based estimations. In the figure is shown the difference in $C I_{95 \%}$ by the model-based method ( \pm 2.44 to $\pm 11.57 \mathrm{Mg} \mathrm{ha}^{-1}$ ) compared to the $C_{95 \%}$ by the samplingbased method $\left( \pm 3.72\right.$ to $\left.\pm 27.61 \mathrm{Mg} \mathrm{ha}^{-1}\right)$. These differences in uncertainty estimation was used to calculate the relative efficiency $(R E)$ for the two periods of study. In $T F$ the $R E$ for 2004-2009 was 5.1 and decreased to 4.7 in the second period. This reduction of $R E$ was also observed in $S M F(6.4$ to 5.4). However, in $S C F$ and oak forest stratum ( $S O F)$ an increase in $R E$ was observed (3.7 to 5.9 in $S C F, 2.3$ to 2.8 in $S O F$ ). 


\section{Chapter V: Discussion}

\section{V.1 Measurement errors}

The first empirical study analyzed measurement errors using the discrepancy between two measurements for 4262 trees. In studies applying the same methodology, Berger et al. (2014) reported similar numbers of sampled trees, with 4411 trees, but generally smaller sample sizes have been used, such as 391 (Kitahara et al., 2010), 879 (Elzinga et al., 2005) and 778 trees (Melson et al. 2001). In the second empirical study, the number of trees was reduced, but the number of repetitions was increased, with five remeasurements in 250 trees of the $D B H$ and $T H$ variables. Similar studies to the second empirical study have been conducted by Luoma et al. (2017) with four measurements and McRoberts et al. (1994) with eight measurements per tree.

The $D B H$ in our first study made in conifer forest had a mean error $(\hat{\sigma})$ with Caliper of $0.36 \mathrm{~cm}$ or a relative standard deviation $(R S D)$ of $2.2 \%$. For our second study in broad-leaved forest, we estimated $\hat{\sigma}$ of $0.19 \mathrm{~cm}(R S D=0.9 \%)$ with Caliper and $0.16 \mathrm{~cm}(R S D=0.8 \%)$ with Tape. These results are consistent with studies that report $R S D$ of $1.6 \%$ with Tape (Elzinga et al., 2005), standard deviations of $0.51 \mathrm{~cm}$ (Melson et al. 2001) and $0.3 \mathrm{~cm}(R S D=1.5 \%)$ with multiple Caliper measurements (Luoma et al., 2017). Using Tape, Kitahara et al. (2010) estimated $\hat{\sigma}$ of 0.37-0.57 cm $(R S D$ of $1.3-2.9 \%)$ for conifer trees and $0.52-0.83 \mathrm{~cm}$ ( $R S D$ of $1.2-3.2 \%)$ for broad-leaved trees.

The second empirical study calculated smaller measurement errors. Each re-measurement was performed by the same individual to obtain the best estimate, i.e. without time pressure and following the field manual. Thus, it was possible to estimate smaller measurement errors than the ones estimated in other studies, like the study of Kitahara et al. (2010), in which measurement errors are estimated comparing the measurements of field crew with measurements made by forest experts. Or similar approaches like in our first empirical study, in which the errors are estimated from measurements made by different field crew members (Elzinga et al., 2005; Liu, Bitterlich, Cieszewski, \& Zasada, 2011; Luoma et al., 2017; McRoberts et al., 1994; Melson et al., 2001). In preparation for error propagation, $D B H$ measurement errors were adjusted to probability distribution functions (PDF) under two criteria. The first was to assume that measurement errors have a single PDF describing them, independently of the size of the tree. Our study found that the PDFs that best-fit for all errors were Johnson SU and Logistic. For both cases, normal distribution $(N D n)$ was also adjusted showing overlapping with the best-fitted PDF. The second criterion was to incorporate the heteroscedasticity of errors into the PDFs. Therefore, a PDF was fitted per $D B H$ class, assuming that within the classes the distribution of errors is the same. It was found that in $D B H$ classes less than $30 \mathrm{~cm}$, the $N D n$ overlapped with the best-fitted PDF. However, beyond 30 $\mathrm{cm} D B H$, the best-fitted PDFs showed differences with the NDn (see Figures IV-4 and IV-9). With respect to selected PDF, most studies assume $N D n$ by reporting the dispersion of errors in $D B H$ through standard deviation (Berger et al., 2014; Kitahara et al., 2010; Melson et al., 2001). Some authors provide support for the use of the NDn (Gertner \& Köhl, 1992; Kangas, 1998), and other studies include heteroscedasticity of errors by calculating standard deviations by $D B H$ class (Elzinga et al., 2005; Liu et al., 2011; Luoma et al., 2017). Logarithmic transformations were also found to be the most commonly used method for describing heteroscedastic errors (Chave et al., 
2004; Kangas, 1998). It should be noted that no work was found using other types of distribution than normal or log normal to describe measurement errors in $D B H$.

In estimating errors for the $T H$ variable, the first study had a mean error $(\hat{\sigma})$ of $0.61 \mathrm{~m}$ or $R S D=4.6 \%$ when measured with Vertex, while in the second study had values of $0.88 \mathrm{~m}(R S D=5.0 \%)$ for Blume-Leiss $(B L)$ and $0.58 \mathrm{~m}(R S D=3.0 \%)$ for Vertex. The results of our first two studies are similar to those reported by (Luoma et al., 2017) with $0.5 \mathrm{~m}$ (RSD of 2.9\%) for Vertex but smaller than the ones estimated by (Melson et al., 2001) of $1.52 \mathrm{~m}$. Also with Vertex measurements, Kitahara et al. (2010) estimated errors of 0.66 to $1.02 \mathrm{~m}$ ( $R S D$ of 3.1-4.5\%) for conifers and from 1.2 to $1.89 \mathrm{~m}$ (RSD of 7.1-9.8\%) for broad-leaved.

Like the errors in $\mathrm{DBH}$, the errors in $\mathrm{TH}$ measurement were adjusted to a normal distribution (NDn) and the PDF that best fit to the error frequencies. The PDFs that best fit the measurement errors in conifers (first study) were Johnson SU and in hardwoods (second study) Cosine and Trapezoidal, for Vertex and Blume-Leiss. The PDF's adjusted for Vertex measurements were closer to NDn than the measurements of Blume-Leiss. When adjusting PDFs by $T H$ classes, it was found that in conifers there was a greater frequency of errors close to zero, prevailing the Johnson $S U$ distribution (see Figure IV-5). In hardwoods, Vertex measurements also had a higher frequency of near-zero errors, and the NDn overlapped the best-fit PDFs in $T H$ less than $15 \mathrm{~m}$ and $T H$ beyond $25 \mathrm{~m}$. The PDF of measurements with Blume-Leiss in hardwoods overlapped with the NDn in the small tree classes. However, from 15 meters of $T H$, there was no high frequency in central values of errors, and the PDFs describing the errors were Trapezoidal Curvilinear and Uniform (see Figure IV-10). The studies reporting measurement error in $\mathrm{TH}$ assume normality in the error distribution (Kangas, 1998; Kitahara et al., 2010; Melson et al., 2001) and even estimate the errors according to the TH classes, as in our study (Luoma et al., 2017; Williams et al., 1994).

In the second empirical study of this thesis, were used measurement errors of students with mean error $(\hat{\sigma})$ of $1.18 \mathrm{~cm}$ for $D B H$ and $4.01 \mathrm{~m}$ for $T H$, using Tape and Vertex. The errors in $D B H$ were similar to those found by Kitahara et al. (2010), that calculated errors for three groups of inexperienced crew members with a range from 0.19 to $1.43 \mathrm{~cm}$ with hardwood species. In contrast, the $T H$ errors from 0.48 to $2.09 \mathrm{~m}$, were smaller to those obtained by the students in our study. We emphasize that the $T H$ range of the species measured by Kitahara et al. (2010) of 4.2 to $22.7 \mathrm{~m}$ with an average of $13.2 \mathrm{~m}$, was lower than our study with 7.3 to $36.3 \mathrm{~m}$ and an average of $20.2 \mathrm{~m}$.

Although measurement errors increase with increasing $T H$, compared to other studies (Luoma et al., 2017; Melson et al., 2001), our mean TH measurement error of students was overestimated.

\section{V.1.1 Measurement and uncertainty comparison among devices}

In the second empirical study, the mean $D B H$ values were larger from Tape than from Caliper $(24.24>23.97 \mathrm{~cm})$. However, the mean measurement errors $(\hat{\sigma})$ were larger with Caliper than with Tape $(0.19>0.16 \mathrm{~cm})$. Other temperate forest studies (Liu et al., 2011; Moran \& Williams, 2002; Weaver et al., 2015) confirm this trend. In relation to larger measurements from Tape, Brickell (1970) and Matérn (1956), explained that the perimeter measured with Tape is assumed from a circumference, being this figure the one that estimates larger surface given a perimeter. Matérn (1956) argued that measurements with Tape are more consistent than with Caliper since Caliper measurement represents one diameter of an infinite number that makes up a cross-section. This 
explains why repeated measurements with Caliper had larger measurement errors compared to Tape.

Mean $T H$ measurements were larger with Blume-Leiss $(B L)$ than with Vertex $(19.97>19.75 \mathrm{~m})$. Measurement errors $(\hat{\sigma})$ ranged from 0.03 to $2.22 \mathrm{~m}(R S D$ of $0.2-18.3 \%)$ and 0.04 to $1.48 \mathrm{~m}(0.4-$ $9.7 \%)$, respectively, with mean errors of $0.88 \mathrm{~m}(R S D=5.0 \%)$ and $0.58 \mathrm{~m}(R S D=3.0 \%)$. The errors in $B L$ were similar to those obtained by Williams et al. (1994) with clinometers; $0.46 \mathrm{~m}$ in trees up to $12.2 \mathrm{~m}$, and $2.22 \mathrm{~m}$ in larger trees. Hyppönen and Roiko-Jokela (1978) estimated also a similar range of 0.56-0.80 m with clinometers Sunnto. However, our errors were larger than West (2009), who found that the accuracy of optical-mechanical equipment (Blume-Leiss) is around $0.5 \mathrm{~m}$. Errors in Vertex measurements were larger than the estimated by Gaudin and Richard (2014) and Wing et al. (2004), calculating errors of 0.19 to $2.3 \%$ in experiments with 10 trees. Our results were also larger than the errors reported by Rondeux and Pauwels (1998), who compared Vertex and $B L$ measurements, estimating $R S D$ of $1-1.6 \%$ for Vertex and $2.3 \%$ for $B L$ and mean errors of $5 \mathrm{~cm}$ for Vertex and $34 \mathrm{~cm}$ for $B L$. Here we emphasize that studies reporting errors in Vertex measurements have been conducted under controlled conditions (e.g. five trees in an urban area), compared to our study where 250 trees were measured in the field.

In the experiments developed in this thesis, it was observed that the measurement errors were higher in $T H$. If we use the measurements $(D B H=\mathrm{cm}$ and $T H=\mathrm{m})$ into an allometric model, these measurements are coefficients with which the AGB is estimated. Comparing them without dimension, the measurement errors in $T H$ were less precise than those of $D B H$ (see Figures IV-4, IV-5, IV-9, and IV-10), similar to the results reported by Kitahara et al. (2010) and Luoma et al. (2017).

\section{V.2 Aboveground biomass \& basal area}

\section{V.2.1 Aboveground biomass (W) allometric models (AM) selection}

From the eight $A M$ used in the first empirical study, five models were selected by species and three by genus (see Table III.1). However, an AGB model for Abies duranguensis (Rojas-García et al., 2015a) has not been reported in Mexico, and due to the similarity in morphological characteristics an Abies religiosa model was used (Avendaño Hernandez et al., 2009). The impact of this bias is considered negligible because there was only one tree out of 4262 in the dataset.

For the third empirical study, Table VIII-1 (Appendix I) shows the list of 36 AMs used to estimate the AGB of the 346 species identified in the MNFI for the two periods studied. Five important features in the selection of $A M s$ are discussed below:

i. $\quad 19$ AMs estimated the AGB grouped by genus. The models used for Quercus spp and Pinus spp stand out, with 77 and 37 species.

ii. Desert communities and tropical dry forest were $A M$ that estimated AGB for 73 genera (20 and 53 genera, respectively). Although these $A M s$ were applied to genera of the same vegetation type, $60 \%$ and $30 \%$ of the trees using these models belong to genera that were not included in the studies where these models were fitted. 
iii. $18 \mathrm{AMs}$ used in this thesis were not fitted in Durango or the surrounding states, and six models were not fitted in Mexico.

iv. Only $16 A M$ were reported together with the $M S E$ of the fitted model but did not include the information used to fit the model.

v. 15 of the $A M s$ used were fitted with information from less than 30 sampled trees.

The first three features are related to the $A M s$ developed in the state of Durango. Pinus spp and Quercus spp trees make up $88 \%$ of MNFI trees, and the commercial importance of these genera account for 89.5 and 6.9\% of timber production in Durango (INEGI, 2016). The compilation of 346 models made by Rojas-García et al. (2015a), has 43 models developed in Durango, 33 of these models were made in Pinus spp (11 species) and seven in Quercus spp (three species). For this reason, tropical and desert species have more interest in neighboring states such as Sinaloa for tropical vegetation (Návar, 2009, 2010) and Sonora for desert vegetation (Búrquez et al., 2010). In our thesis work, the need for allometric models involving information from Pinus cembroides, Quercus eduardii, Q. magnoliifolia, $Q$. laeta and $Q$. grisea has been detected. Therefore, a clear adherence to procedures indicating the precision to obtain allometric models is suggested (Picard et al., 2012). These species are $18.6 \%$ of the trees in our study area and have not been reported models in Durango or neighboring states. Some of these species have also been detected as an area of opportunity in $A M$ research, in studies such as that conducted by Rojas-García et al. (2015b), listing 40 priority species for the development of $A M$ in Mexico.

The fourth feature is that $A M s$ have been reported mostly with the determination coefficient $\left(R^{2}\right)$ only and not with the goodness-of-fit. Rojas-García et al. (2015a) reported that out of 346 AMs compiled from studies conducted in Mexico, only 39 models reported the MSE of the fitted model. The models used in our thesis did not include the original data in their publications, with which the error of prediction of the mean and the error of prediction of the individual are estimated (Draper \& Smith, 1998). Since the original data were not available, the $M S E$ was used as the uncertainty parameter due to $A M$, which corresponds to an overestimation of the prediction of the mean or $S E$ (Yanai et al., 2010).

The fifth feature is associated with the information that was used to produce the $A M s$, i.e. the number of trees used to estimate the $A M$. Using less than 30 trees, according to Picard et al. (2012), assumes homogeneity of the species in a 10 ha sampling stand. In addition, Chave et al. (2004) found that increasing the sample size decreases the coefficient of variation in the estimated $A M$. This implies that models with smaller sample sizes (less than 20 trees) may have greater uncertainty in the estimation of the AGB (Roxburgh et al., 2015). However, AMs that used less than 20 trees for their fitting, were still used in our thesis, when no other publications were found for that genus or species (Rojas-García et al., 2015a). It should be noted that this decision is considered to have a negligent impact on our AGB estimate because the AMs fitted in Durango State had sample sizes from 30 to 423 trees and were applied in $96 \%$ of the dataset.

On the variables used in the $A M s, 23$ of the selected models were fit with $D B H$ data and 13 models added $T H$ in the model fitting. Vargas-Larreta et al. (2017) found that for $A M s$ of pines and oaks, $T H$ was a significant predictor variable, improving the prediction of adjusted $A M s$ in 12 of the 17 that were included in their study. This same conclusion has been found by other studies with pines 
and eucalyptus (Bartelink, 1996; Reed \& Tomé, 1998). In a sweet chestnut forest, MenéndezMiguélez et al. (2013) found an increase in the accuracy in AGB estimation including TH. The 13 models including $T H$ as a predictor variable in our study were used in AGB's estimate of $96.8 \%$ of the MNFI dataset.

An important consideration in the $A M s$ selection is the correct identification of the sampled trees in the field. Although our thesis did not include an experiment that quantified the success in the identification of trees, it is explained below how this topic was approached. In the first empirical study, the identification of the trees is considered correct, because the members of the field crew were selected for their experience in the study area as suggest the NFI reviewed by (Tomppo et al., 2010). For the second empirical study, conifer and broad-leaved AMs were assigned, which are distinct vegetation groups. In the third empirical study, the trees in the MNFI database are assumed to be correctly identified according to the field manual (CONAFOR, 2009b). However, a CONAFOR study, which aimed to verify the identification of MNFI species from 2013-2015, obtained results that contrast with the assumption of correct identification (Ricker et al., 2015). This study had 14035 samples, out of which $69.2 \%$ were identified in terms of species (9711 samples) and $30.8 \%$ (4324 samples) were identified in terms of the genus, family or unknown. A group of 47 biologists determined that $39.3 \%$ of the 9711 records were correctly identified, $28.1 \%$ were correct at the genus level, and 32.6\% were misidentified. From 4324 collections, 1856 were not identified with traditional methods and required the use of molecular methods. Therefore, based on this study, $60.6 \%$ of the collections were correctly identified at the genus level, while only $27.2 \%$ were correct at the species level. In personal communication with Dr. Martin Ricker, the researcher responsible for the project, $89.9 \%$ of the collections coincided at the genus level in the state of Durango. From this information, it was decided to use the $A M s$ at the genus level for the estimation of the AGB in the MNFI.

\section{V.2.2 AGB estimation}

In the Durango state, two empirical studies from this thesis estimated AGB. In the first study, the AGB in pine forest was $176.07 \mathrm{Mg} \mathrm{ha}^{-1}$ and ranged from 86.61 to $228.41 \mathrm{Mg} \mathrm{ha}^{-1}$. This estimate contrasts with the national estimate in Mexico of $63.43 \mathrm{Mg} \mathrm{ha}^{-1}$ for this forest type (CONAFOR, 2017a). The major difference was found in tree density and tree height. While in the first study the average values were 1137 trees $\mathrm{ha}^{-1}$ and $13.3 \mathrm{~m}$ of $\mathrm{TH}$, at the national level, 455 trees ha ${ }^{-1}$ and a 6.7 $\mathrm{m}$ of $\mathrm{TH}$ (CONAFOR, 2014a). On the other hand, the estimate of AGB was consistent with the study conducted by Vargas-Larreta et al. (2017), where they estimated $129.84 \mathrm{Mg} \mathrm{ha}^{-1}$ in a range of 11.06 to $469.42 \mathrm{Mg} \mathrm{ha}^{-1}$ for pine and mixed forests in Durango.

In the third empirical study, AGB in the temperate forest of Durango was estimated for two periods of the MNFI data. The mean AGB was 64.31 $\mathrm{Mg} \mathrm{ha}^{-1}$ for the period 2004-2009 and $64.77 \mathrm{Mg} \mathrm{ha}^{-1}$ for 2009-2014. This AGB value lies intermediate to the estimates made in Durango ranging between 48.86 and $130 \mathrm{Mg} \mathrm{ha}^{-1}$ from 2008 to 2012. However, those estimates were the result of different sampling designs and approaches, such as circular plots (Návar, 2009), permanent plots of 50 per 50 m (Martínez Barrón et al., 2016; Vargas-Larreta et al., 2017), or technical studies that calculate AGB from volume estimates (Profloresta, 2008). The National Forestry Commission of Mexico has not reported AGB in the last report of the state (CONAFOR, 2014a), and with MNFI data, the AGB reported to FAO was 54.08 and $54.11 \mathrm{Mg} \mathrm{ha}^{-1}$ in 2007 and 2011 for temperate forest 
(FAO, 2015). In our study, using MNFI data, the temperate forest registered larger AGB estimates in Durango State than at the National level in Mexico.

Inside the Durango temperate forest in the period 2004-2009, the mean estimate of AGB was 48.31 $\mathrm{Mg} \mathrm{ha}^{-1}$ in the conifer forest, $77.48 \mathrm{Mg} \mathrm{ha}^{-1}$ in the mixed forest, and $35.52 \mathrm{Mgha}^{-1}$ in the oak forest. In the second period (2009-2014), the mean values of AGB were 51.82, 73.12, and $36.84 \mathrm{Mg} \mathrm{ha}^{-1}$, in the same order. These estimates could only be compared with the MNFI 2009-2014 report because it was the first report including the AGB estimate. In this report, the AGB was $63.43 \mathrm{Mg}$ $\mathrm{ha}^{-1}$ in the conifer forest, $53.66 \mathrm{Mg} \mathrm{ha}^{-1}$ in the mixed forest and $34.25 \mathrm{Mg} \mathrm{ha}^{-1}$ in the oak forest. AGB in the oak forest was similar to that reported at the national level of Mexico. Conversely, our study estimated lower AGB in the conifer forest, but higher AGB in the mixed forest. This opposite behavior was caused since the mixed forest in Durango had more trees than the conifer forest (487>409), and higher mean $D B H(16.94>16.75 \mathrm{~cm})$.

In the second empirical study, conducted in Göttingen, the average AGB was $257.6 \mathrm{Mg} \mathrm{ha}^{-1}$ in the beech forest. High accumulation of AGB is common in this area and has previously been registered by Brumme \& Khanna (2009) with estimates of $431 \mathrm{Mg} \mathrm{ha}^{-1}$ in a range of 128 to $660 \mathrm{Mg} \mathrm{ha}^{-1}$ from a study conducted in the "Göttinger Wald".

In all three empirical studies, the categories of $D B H$ greater than the category of the mean $D B H$ had a greater contribution to AGB. In first and third studies in Durango, trees with more than $20 \mathrm{~cm}$ (26 and $32 \%$ of the dataset, respectively) contributed to more than $58 \%$ of the AGB. In Göttingen, trees greater than $40 \mathrm{~cm}$ in $\mathrm{DBH}$ (22\% of the dataset) contributed to $65 \%$ of the AGB. The categories greater than or equal to the category of the average $D B H$ had fewer trees but a greater contribution to the AGB.

\section{V.2.3 Basal area $(G)$ estimation}

The $G$ estimate in the first empirical study had the mean value of $29.17 \mathrm{~m}^{2} \mathrm{ha}^{-1}$ in pine forest, which is consistent with the result estimated in the same area of Durango (UMAFOR 1008, see Figure II2) by Molinier et al. (2016) estimating $23.44 \mathrm{~m}^{2} \mathrm{ha}^{-1}$, with a range of 8.21 to $54.83 \mathrm{~m}^{2} \mathrm{ha}^{-1}$. At the state level, the third empirical study had estimates of 12.98 and $13.17 \mathrm{~m}^{2} \mathrm{ha}^{-1}$ for the temperate forest, for the 2004-2009 and 2009-2014 periods. At the national level, the results were similar with 11 and $14 \mathrm{~m}^{2} \mathrm{ha}^{-1}$ for the same periods (CONAFOR, 2012c, 2017a); and also similar to the estimate for the state of Durango in 2013 with $10.77 \mathrm{~m}^{2} \mathrm{ha}^{-1}$ (CONAFOR, 2014a). At the state level of Durango, the third empirical study recorded $1.7 \%$ of $R S E$ for the two periods studied, being consistent with the estimate of MNFI 2009-2014 with 1.98\% of RSE, and with 2.2\% estimated for this state in 2013.

In the second empirical study, a $G$ value from 29 to $32 \mathrm{~m}^{2}$ ha ${ }^{-1}$ with a $R S E$ of $5.9 \%$ was estimated; similar results were obtained in the monitoring reported by Brumme \& Khanna (2009) from 32.3 to $39.5 \mathrm{~m}^{2} \mathrm{ha}^{-1}$ in beech forest near the study area.

The contribution to the $G$ estimates from bigger trees, $D B H$ larger than the category of the mean $D B H$, was large. In the first empirical study, trees larger than $20 \mathrm{~cm} D B H$ were $26 \%$ of the dataset and contributed $54 \%$ of the $G$ estimate. In the second empirical study, same $D B H$ trees size were the $32.5 \%$ of the dataset, contributing $59.2 \%$ of $G$. This contribution of bigger trees was also recorded in the MNFI 2009-2014, where trees larger than $20 \mathrm{~cm} \mathrm{DBH} \mathrm{(21 \%} \mathrm{of} \mathrm{the} \mathrm{dataset)} \mathrm{contributed} \mathrm{56 \%}$ 
of the $G$ estimation (CONAFOR, 2017a). As in the Durango estimates, the large trees of the second empirical study (DBH larger than $40 \mathrm{~cm}$ and $22 \%$ of the dataset) contributed $60 \%$ of the estimated $G$.

\section{V.3 Error propagation}

\section{V.3.1 Tree-level (measurement contributions)}

The contribution of measurement errors (uMes) in $D B H(u D B H)$ and $T H(u T H)$ were the first sources of uncertainty in the estimation of AGB at tree-level. With the GUM Method, the contribution of uncertainties $u D B H$ and $u T H$ were estimated for each tree (uMes) in the first two empirical studies (Equations $06 a$ and $06 b$ ). The major contribution in the measurement errors at the tree-level was the $u T H$, followed by $u D B H$ and finally the correlation of these two variables $\left(u \rho_{(D B H, T H)}\right)$.

The contribution of the $u T H$ was higher due to the following reasons: the relationship between the sensitivity coefficients $S C_{D B H} / S C_{T H}=1.65$ (Equation 05) indicated that in the allometric models used, for each unit of $u T H$, the $u D B H$ contributed $65 \%$ more. However, because the variables $(D B H, T H)$ are correlated, the contribution of the correlation was incorporated in the error propagation; thus, the contribution of the $u D B H$ decreased, and consequently the ratio $u T H / u D B H$ favored $u T H$, having contributions from 37 to $62 \%$ to the measurement errors. Chave et al. (2004) found also more contribution of the $u T H$ to the measurement error in tropical forest, estimating 48 to $78 \%$ form $u T H$; as well as Phalla et al. (2018) who estimated a contribution of $74 \%$ from $u T H$ to the measurement errors in evergreen forest.

The NDnC scenario (description in Section III.1.6.4) included the heteroscedasticity of measurement errors, and it was not significantly different from the other two scenarios used ( $N D n$, $R M S D)$. Because these scenarios underestimated ( $N D n)$ and overestimated (RMSD) the measurement errors calculated by $N D n C$; the $N D n C$ scenario, using tree size, describes best the errors from the discrepancies in $\mathrm{DBH}$ and $\mathrm{TH}$ measurements in the study area of El Salto, Durango.

The second empirical study was designed to compare the measured error from repeated measurements per tree, against an estimated parameter from the application of four scenarios of estimation. The scenarios that showed no significant difference with the measured errors were linear model $(\mathrm{mod})$ and normal distribution $(N D n)$. Applying the scenarios in the two datasets, Caliper+Blume-Leiss $(C B)$ and Tape+Vertex (TV), the largest contribution was from $u T H$, followed by the correlation $(D B H, T H)$, and the smallest was from $u D B H$. The largest contribution of $u T H$ was already mentioned for the first empirical study. The correlation of the second empirical study was greater than in the first empirical study $(\rho=0.92>\rho=0.78)$, and we assume that for this reason there is a greater contribution of the correlation in relation to the $u D B H$. Tiedeman \& Green (2013) found that including the correlation between observations affects the uncertainty of the source involved in the correlation. In general, the correlation between variables affects uncertainty estimation (Criscenti et al., 1996; Farrance \& Frenkel, 2014; Goliaš \& Palenčár, 2012; Mahmoud \& Hegazy, 2017). The results of our thesis show a range from 13 to $30 \%$ of the tree-level uncertainty comes from correlation. However, in the AGB estimation, the contribution of correlation uncertainty of the measurement variables has not been reported. Estimates have been reported with 
allometric models using only $D B H$ as a predictor variable (Qin et al., 2019), or assuming independency of the DBH and TH measurements (Berger et al., 2014; Holdaway et al., 2014; McRoberts \& Westfall, 2014; Phalla et al., 2018; Phillips et al., 2000; Van et al., 2011).

The AGB and their uncertainty, estimated with models fitted with the variables $D B H$ and $T H$, were compared with estimates from models fitted with $D B H$. Models using only $D B H$ underestimated the AGB, and it is significantly different from estimates by the models using $D B H$ and $T H$. This was a clear difference between the two models and was previously stated in Section V.2.1. The AGB estimation models using only the predictor variable $D B H$, had less uncertainty at tree-level, compared to the model that used $D B H$ and $T H$. This larger uncertainty when using allometric models with $T H$ has been reported by Phalla et al. (2018) and Chave et al. (2004). In these studies, they attribute more difficulty in measuring $T H$ as the reason for the increased uncertainty, and as we have seen in this thesis, this was observed in the devices used (Section V.1.1).

Because we used two devices per measured variable in the second empirical study, we compared the uncertainty contributions. The $C B$ has a larger contribution, compared to $T V$, to the measurement error at tree-level. This larger contribution is associated with the uncertainty parameters used in error propagation (Table IV-8), which show a greater magnitude of both error parameters of the $C B$ dataset, $u_{\text {Caliper }}>u_{\text {Tape }}$, and $u_{\text {Blume-Leiss }}>u_{\text {Vertex }}$.

\section{V.3.2 Tree-level (non-sampling error)}

In the GUM Method, the non-sampling error (uTree) was estimated (Equation 07) with the contributions of measurement errors (uMes) and prediction errors $(u A M)$. The first two empirical studies represent two scenarios not only geographically, but also in the contribution of $u A M$ to the uTree.

In the first study, the $u A M$ contributed to more than $97.8 \%$ to the $u N S$ estimated at tree-level; the uMes had a range of 8.79 to $13.18 \mathrm{~kg}$ and the $u A M$ was $87.42 \mathrm{~kg}$ for the $u A M$ of the average tree. Similar results were obtained by Phalla et al. (2018) with $u A M s$ contribution of 81 to $98 \%$, and more conservatively by Berger et al. (2014) with less than $83 \%$ of $u A M$ contribution. On the contrary, our second study showed a greater contribution of the uMes (more than 98.9\%) to the uTree. The uMes had a range of 12.16 to $18.26 \mathrm{~kg}$ and $u A M$ was less than $1.35 \mathrm{~kg}$ per tree.

Holdaway et al. (2014) had similar results where $u M e s$ contributed to more than 10 times the $u A M$, using the standard error of the mean as the source of uncertainty of the carbon estimation model.

The previous information allows us to understand the difference in contributions of the errors included in the $u T r e e$ for the first two empirical studies. This difference is related to the model selection or the source of prediction error used (RMSE or $S E$ ) as mentioned by Holdaway et al. (2014) (discussed in Section V.2.1); however, it has another implication in the aggregation process. In the first empirical study, having a $u A M>u M e s$ relation in the uncertainty at tree-level, $97.8 \%$ was due to $u A M$ and therefore the size of the tree is not relevant. When estimating the AGB for trees beyond $20 \mathrm{~cm} D B H$, although $59 \%$ of the AGB was calculated, the $26 \%$ of the $u N S$ is estimated and is the same percentage of trees of this $D B H$ size. In the second study, with an inverse relationship $u M e s>u A M$, the uncertainty at tree-level was $98.9 \%$ from $u M e s$. When verifying it with the dataset measured by Caliper+Blume-Leiss, it was found that trees of $D B H$ greater than $40 \mathrm{~cm}$, account $78 \%$ of the AGB and $65 \%$ of the $u N S$; even when it is the $22 \%$ of the trees in the Stand. 


\section{V.3.3 Plot-level (non-sampling error)}

Adding the non-sampling errors per tree (uTree) to estimate the non-sampling errors per plot (uNSplot), the proportion of errors decreased. Using the results of the first study, the relative errors (uTree/AGB) at tree-level were on average 56.7 to $57.4 \%$. In plot-level, the range of relative errors (uNSplot/AGB per plot) was from 1.32 to $6.09 \%$ (see Table VIII-8, Appendix IV). The reduction of percentage in the aggregation is because the sum of the errors is in quadrature. The sum of errors, random and independent, is smaller than the sum and can be described by the geometric form of the right-angled triangle (Taylor, 1997). In this triangle, the legs represent the $u$ Tree and the hypotenuse is the sum in quadrature of the two uTree; with a greater contribution from the larger uTree. The sum of two uTree (hypotenuse) is the leg of a next triangle adding the next uTree, and so on. The same result is obtained using Equation 08 applied to all the $u$ Tree. The above also means that trees with larger $u$ Tree have a greater contribution to the uNSplot.

The reduction of relative errors, from tree to plot, was also observed in the second empirical study with a smaller difference. For Caliper+Blume-Leiss measurements, the relative errors in tree-level were from 3.56 to $3.96 \%$ and in plot-level from 0.73 to $1.50 \%$ (see Table VIII-26, Appendix V). In Tape+Vertex the same trend was found (see table VIII-29, Appendix V). The lower contribution of measurement errors in Vertex, which had already been explained, maintained a lower contribution in errors at the plot-level.

\section{V.3.4 Stand-level GUM Method (non-sampling \& sampling errors)}

At the stand-level, unlike tree-level and plot-level, the uncertainty was also estimated with the Monte-Carlo simulation Method (MCM). The RSE of 7.88\% is high, according to the recommendation of $R S E<7 \%$ proposed by Velasco et al. (2002); however, the experiment met the purpose of estimating the parameters of measurement errors. With the results of the GUM Method, the $u N S$ for the stand was obtained by applying Equation 09 to the uNSplot. The first empirical study resulted in a mean AGB estimate of $176.07 \mathrm{Mg} \mathrm{ha}^{-1}$ and a total uncertainty $(u W)$ of $13.87 \mathrm{Mg}$ $\mathrm{ha}^{-1}$. The $u N S$ contributed to $1.26 \%$ of this total uncertainty when using the $N D n C$ scenario. In the second empirical study, the $C B$ dataset had a mean AGB estimate of $236.28 \mathrm{Mg} \mathrm{ha}^{-1}$ and $u W$ of $13.74 \mathrm{Mg} \mathrm{ha}^{-1}$. In the $T V$ dataset were estimated a mean AGB of $236.28 \mathrm{Mg} \mathrm{ha}^{-1}$ and $u W 14.43 \mathrm{Mg}$ ha $^{-1}$. The $u N S$ contributed to $0.40 \%$ of $u W$ in the $C B$ dataset and $0.15 \%$ in the $T V$ dataset. These results indicate that the $S E$ contribute to over $98.7 \%$ in the first two empirical studies when estimated with the GUM Method for error propagation.

Contributions by a source of uncertainty, in the GUM Method, only for the first empirical study were estimated. In the $N D n C$ scenario, the order of contribution was $S E>u A M>u T H$ $>u D B H>u \rho_{(D B H, T H)}$, with percentages of $98.74>1.213>0.02>0.014>0.013 \%$ of the total uncertainty. We observed that the major source of uncertainty for the estimation of AGB in El Salto, Durango was the SE. Similarly, the $u A M$ was more than $97 \%$ of the $u M e s$; and the $u T H$ had the greatest contribution in the $u M e s$, taking into account its relationship with the other measurement errors used at tree-level $\left(\hat{\sigma}_{T H}>\hat{\sigma}_{D B H}>u \rho_{(D B H, T H)}\right)$. 


\section{V.3.5 Stand-level MCM (non-sampling \& sampling errors)}

In each empirical study, we ran 10000 iterations. The resulting differences in the approximations of $M C M$ with the direct estimation were less than $0.1 \%$ when the IPCC recommendation is $1 \%$ (IPCC, 2006). This implies that the results obtained with the $M C M$ were acceptable. The estimated $R S E$ was 7.8, 6.0 and 3.0\%, for the first, second and third empirical studies. Even when the empirical studies were not conducted in the same study area, the RSE show the effect of the sample size (Cochran, 1977) of 10, 47 and over 1600, respectively. The RSE of the third study is comparable to the $R S E$ of $3.3 \%$ reported in the MNFI for temperate forest (CONAFOR, 2017a).

Using the $M C M$, the largest contribution to total uncertainty $(u W)$, for the three empirical studies was the $S E$ with values over $98.7 \%$. Phillips et al. (2000) reported the $S E$ as the most important contribution in volume estimates in South-eastern NFI USA, with over $89 \%$ of the $u W$. Similar results have been reported by Holdaway et al. (2014) and McRoberts \& Westfall (2016) in temperate forest carbon estimation, but do not report the contribution percentage. In contrast, Chave et al. (2004) in a rainforest found a contribution of about $50 \%$ of the $S E$ to the $u W$. This indicates that the number of trees sampled in our studies is large enough so that the major source of error is attributed to sampling (McRoberts \& Westfall, 2016; Phillips et al., 2000). According to McRoberts \& Westfall (2016), with a mean density of 23 trees in $400 \mathrm{~m}^{2}$ plot (575 trees ha ${ }^{-1}$ ), the uMes are negligible comparing to the $S E$. Our results confirm this last statement with a mean value of 20 trees in a $400 \mathrm{~m}^{2}$ plot.

When comparing the results of $u N S$ in the MCM and the GUM Method, in the first empirical study, a slight overestimation of the GUM Method was found. Using the same scenarios (NDn and NDnC) and comparing at plot-level the mean error estimate, resulting in an overestimation of $2 \%$ of the GUM Method (p<0.01, Tables VIII-14 and VIII-16 in Appendix IV). Assessments in instrumentation, and material quality controls, report a range of $8-21 \%$ of overestimate of GUM Method results (Mahmoud \& Hegazy, 2017; Sana Sediva et al., 2015; Sona Sediva \& Havlikova, 2013). However, when we applied the best-fit distribution with the MCM (scenarios $M C B D \&$ $M C B D C$ ) the $u W$ per plot was not significantly different from the GUM Method results (scenarios $N D n$ and $N D n C)(\mathrm{p} \geq 0.614$, Table VIII-16 in Appendix IV). Farrance \& Frenkel (2014) had similar results with no difference between these two methods, assuming independence in the variables used for the error propagation.

In the second empirical study, it was observed that the $u N S$ decreased in proportion more than the $S E$, for all the scenarios evaluated when the sample size increased from 11 to 47 plots. This is evident from Caliper+Blume-Leiss (CBmod scenario) results, in which the $u N S$ of the 11 plots equaled $0.4 \%$ of the $u W$, while in 47 plots was $0.1 \%$. This behavior is explained due to the $G U M$ Method used in the 11 plots, overestimate the $M C M$ results in $u W$ for the 47 plots, and according to Cochran (1977) \& Taylor (1997), the $S E$ and $u N S$ decrease when sample size increase.

The order of the contributions to the $u W$, of the scenarios used in the second study, were $B D<\bmod <B D C$. The scenario mod did not have significant differences in comparison with the measured errors, and mod includes the heteroscedasticity of the measurement errors related to the size of the tree. Then, the other two scenarios underestimated $(B D)$ and overestimated $(B D C)$ the measurement errors. 
In the second study, the contribution obtained with the use of errors made by students (Exp and Nexp) stands out since these were the only scenarios that increased the total RSE from 5.0 to 5.04\%. The Exp and Nexp scenarios represent a six-fold increase in $D B H$ measurement errors, and a 4.5fold increase in $T H$, compared to the estimated errors in repeated measurements. However, when these measurement errors are applied in the third empirical study, they are negligible with the large sample size (NFI scale) as stated by McRoberts \& Westfall (2016).

\section{V.3.5.1 MCM per source contribution}

The results of the $M C M$ in the first empirical study maintained the order of contribution from the sources of uncertainty, where $S E>u A M>u T H>u D B H$. The percentage of contributions per source was $98.746>1.205>0.029>0.028$ and is similar to the estimated with the GUM Method.

In the second empirical study, The sources of uncertainty had a contribution to $u W$ in the following order $S E>u T H>u D B H>u A M>u \rho_{(D B H, T H)}($ see Table VIII-41, Appendix V). The contribution for Caliper+Blume-Leiss measurements with $\bmod$ scenario was 99.9>0.08>0.02>0.0002>0.00002, while with Tape+Vertex measurements was 99.95>0.036>0.014>0.0002>0.00001.

In the third empirical study $(M N F I)$, the order of the contribution to the total uncertainty $(u W)$ was like the one obtained in the first study, but also included the uncertainty of the plot design (uPlot). The contribution to the $u W$ was related to the scenario of measurement error estimation. The scenarios $B D$ (best-fitted distribution) and Exp (experimented students), do not consider the size of the trees and, therefore, the contribution to the final error depends on the number of trees. In contrast, the $B D C$ scenario (best-fitted distribution by class) estimated the contributions according to the size of the tree, furthermore, this scenario did not differ significantly from the other scenarios. Thus, the $B D C$ scenario does not underestimate (as $B D$ ) or overestimate (as $\operatorname{Exp}$ ) the measurement errors in the estimation of AGB. In the $B D C$ scenario, the order of contribution to the $u W$ was $S E>u A M>u T H \geq u P l o t \geq u D B H$. The percentage of contributions per source in MNFI of Durango was $99.41>0.53>0.03 \geq 0.02 \geq 0.02$. The contributions from $u T H, u P l o t$ and $u D B H$ sources were small $(\geq 0.7 \%)$ and did not differ significantly from one another. The $u$ Plot is uncertainty related to the shape and size of the plot, in this study its contribution was equal for the given scenarios. The contributions of $u T H$ were larger than $u D B H$ as the results in the first study, with no significant difference.

Using information from FIA in the South-eastern USA, Phillips et al., (2000) estimated the contributions to total carbon estimation from four sources of uncertainty. The order of the contributions is like that observed in the first and third empirical studies of this thesis, being $S E>u A M>u T H>u D B H$. The reported contribution percentages were 98.7>1.2>0.1>0.0. On the other hand, Holdaway et al. (2014), report a different order of contribution being $S E>u M e s>u A M$ where they estimate a larger contribution from $u M e s$ compared to $u A M$. The percentage contributions were 98.9>1.0>0.09 and, within the measurement errors, the $u T H$ were larger than the $u D B H$. This last is like our results in the second empirical study.

Our thesis results include the contributions of measurement, prediction, plot design and sampling uncertainties in the AGB estimation with the MNFI data for the state of Durango, Mexico. The uncertainty estimates of this thesis were made under the IPCC guidelines established for Tier 2 (IPCC, 2006), which imply the reporting of transparent, coherent, compatible, exhaustive and 
precise estimates (Morfín Ríos et al., 2015). Since AGB estimation is a basic input for the estimation of GHG emissions (CONAFOR, 2014b; Gibbs et al., 2007), it is proposed that the approach used here could be considered in the development of MRV systems under REDD+ in Durango, Mexico. The MRV system in State-level (Durango strategy is under construction) aims to monitor, verify, and adapt national REDD+ strategies, incorporating practices best suited to regional characteristics (CONAFOR, 2017b). The State-level MRV system takes on greater importance since it is requested in reports of environmental management in Mexico, referring to climate change mitigation (SEMARNAT \& INECC, 2017), forest carbon dynamics (Red Mex-SMIC, 2015), and including the Law of Climate Change (Chamber of Deputies, 2018b).

\section{V.3.5.2 Uncertainty in strata and substrata of MNFI}

Estimating AGB by vegetation type is a recommended method to stratify the forest for the AGB uncertainty report, according to the IPCC (IPCC, 2006). Stratification is recommended to reduce variability in estimates; however, the results of our study show the opposite trend. While in the temperate forest the $R S E$ was $3.0 \%$, a range from 3.3 to $10.63 \%$ of $R S E$ was estimated in the strata and from 3.6 to $23.5 \%$ in substrata. The $R S E$ estimates for wood volume in Durango, with information of the MNFI (2009-2014), have an RSE range from 6.97 to $29.71 \%$ for temperate forest strata (CONAFOR, 2014a). In our study, the highest RSE (23.5\%) was estimated in the substratum of secondary vegetation in the conifer forest $(S C F S)$. This substratum has the smallest number of sampled plots $(\mathrm{n}=60)$. In contrast, the lowest $R S E(3.6 \%)$ was in the primary vegetation of mixed forest $(S M F p)$. This is the substratum with the largest number of sampled sites $(\mathrm{n} \geq 1261)$. The increase in measurement errors (scenarios) did not change the contribution of $S E$ and $u N S$ to the $u W$, since both estimates depend on the sample size (Cochran, 1977; Taylor, 1997). However, by stratifying, the contribution of the $S E$ was reduced gradually; while in the temperate forest the $S E$ was $\geq 99.29 \%$, at the stratum level it was $\geq 97.49 \%$ and at the substratum level $\geq 96.39 \%$. The scenarios were consistent in estimating uncertainties as in the temperate forest, with an underestimation of $u N S$ by the scenario $B D$ (best-fitted distribution) and an overestimation of Exp (experimented students), compared to $B D C$ (best-fitted distribution by class).

The number of clusters changed in the strata and substrata of the temperate forest and were not consistent in the periods studied. The classification of vegetation is fundamental information where the field crew applies the NFI field manual (CONAFOR, 2017a; Tomppo et al., 2010). Since classification is qualitative, as well as species identification, Morrison (2016) suggest that experience and training is required for the field crew. The difference is that classification can be only made in fieldwork and trees can be identified in the herbarium, if not possible in the fieldwork (CONAFOR, 2009b; Ricker et al., 2015). The professional profile or experience of the field crew in MNFI is not defined in the field manual. We suggest applying the findings of Tomppo et al. (2010) about the strategies used in the NFI field manuals of 37 countries to ensure the quality of MNFI information. Among these strategies are, the profile of the field crew (forest engineer or technician), training and training evaluation, cross-checking at fieldwork, a random check of the field crew, correction and validation of fieldwork (Tomppo et al., 2010). 


\section{V.4 AGB and basal area estimation with Landsat imagery information}

The basal area $(G)$ and AGB $(W)$, calculated with the MNFI data, were correlated with the information extracted from the Landsat imagery (predictor variables). The predictor variables $(P V)$ with the highest Pearson's correlation coefficient $(|\rho|)$ were $S R, S R G, N D V I, A R V I$, and WDRVI (see Table III-10 for acronym description). The coefficients range for $G$ were from 0.74 to 0.79 and for $W$ from 0.74 to 0.77 . Similar coefficients $(0.74 \leq \rho \leq 0.77)$ were estimated with MNFI data $(G$ and $W)$ by Pérez Miranda et al. (2018) in a mixed forest of Durango for NDVI and GNDVI (Green Normalized Difference Vegetation). Muñoz-Ruiz et al. (2014), also for NDVI and GNDVI, calculated correlations of less than 0.50 in $G$ estimations in a temperate forest of Hidalgo, Mexico.

\section{V.4.1 Models of basal area $(G)$ estimation}

The fitted model to estimate $G$ with predictor variables $(P V)$ from Landsat 5 of 2007, had an $R^{2}$ of 0.58 , an $R M S E$ of $4.33 \mathrm{~m}^{2} \mathrm{ha}^{-1}$, and a $C V$ of $33.8 \%$. With $P V$ from Landsat 8 of 2013 , the $G$ model had an $R^{2}$ of 0.64 , an $R M S E$ of $4.11 \mathrm{~m}^{2} \mathrm{ha}^{-1}$ and a $C V$ of $32.0 \%$. The results of this thesis are similar to those obtained by Muñoz-Ruiz et al. (2014) and Pérez Miranda et al. (2018) in a temperate forest, combining information from the MNFI, with SPOT and Landsat 7 imagery, respectively. In these studies, they estimate $R^{2}$ of 0.32 and 0.58 , and $R M S E$ of 4.70 and $3.85 \mathrm{~m}^{2} \mathrm{ha}^{-1}$. However, Günlü et al. (2014) estimate smaller $R M S E=1.74 \mathrm{~m}^{2} \mathrm{ha}^{-1}$, in a smaller study area $\left(180 \mathrm{~km}^{2}\right)$ of conifer forest in Turkey.

\section{V.4.2 Prediction models of AGB $(W)$}

Models for $W$ prediction, fitted with $P V$ from Landsat 5, had $R^{2}$ of $0.60, R M S E$ of $24.5 \mathrm{Mgha}^{-1}$ and $C V$ of $40.5 \%$. Using $P V$ from Landsat 8 were estimated $R^{2}$ of $0.63, R M S E$ of $23.9 \mathrm{Mgha}^{-1}$ and $C V$ of $39.6 \%$. In the temperate forest of Durango, $W$ estimation has been studied combining field sampling and remote sensing, under different characteristics. Pérez Miranda et al. (2018), estimated similar results with $R^{2}$ of 0.59 and $R M S E$ of $21.65 \mathrm{Mgha}^{-1}$, using MNFI and Landsat imagery in mixed Forest of Durango. Martínez Barrón et al. (2016), using data from permanent plots estimated $W$ combined with Landsat in the temperate forest of Durango, and obtained an $R^{2}$ of 62.41, but higher $R M S E$ of $54.74 \mathrm{Mgha}^{-1}$. With a lower $R^{2}$ of 0.46 and higher RMSE of $49.77 \mathrm{Mgha}^{-1}$, Vargas-Larreta et al. (2017) estimated $W$ using permanent plots and Landsat imagery in Durango. In contrast, López-Serrano et al. (2019) estimated an $R^{2}$ of 0.80 and lower $R M S E$ of $8.20 \mathrm{Mgha}^{-1}$, in a $W$ estimated a range from 1.72 to $101.71 \mathrm{Mgha}^{-1}$; smaller range compared with the estimated in our study, from 0.23 to $457.04 \mathrm{Mgha}^{-1}$. Two aspects can be distinguished which differentiate the studies carried out in Durango compared to this thesis work. The first is the sampling design in our thesis, covering the total temperate forest area (see Figure II-4); compared to sampling designs that partially cover the study area, in the other studies showed. The second aspect is in favor of permanent plots, designed for the analysis of growth, production and evolution of forest stands through site monitoring. These plots are evaluated every three to five years (Corral-Rivas et al., 2009). In addition, these plots have a larger area $\left(2500 \mathrm{~m}^{2}\right)$ of sampling compared to the MNFI plots $\left(1600 \mathrm{~m}^{2}\right)$, which decreases the uncertainty in the $W$ estimation according to Chave et al. (2004). 


\section{V.4.3 Basal area $(G)$ and AGB $(W)$ estimation in strata of temperate forest}

The models fitted at the strata-level showed differences in the Godness-of-fit and coefficient of determination $\left(R^{2}\right)$ compared with the models fitted for the temperate forest. In conifer forest, the $R^{2}$ and $C V$ increased in both estimated variables, $G$ and $W$. The $R^{2}$ had values of more than 0.64 , while $C V$ was more than $37.6 \%$ in $G$, and more $46.9 \%$ in $W$. In mixed forest, for both estimates, the $R^{2}$ decreased to less than 0.62 , and the $C V$ also decreased to less than $36.7 \%$. The models to estimate $G$ and $W$ in the oak forest had the lowest $R^{2}$ of less than 0.42 and the $C V$ increased to more than 39 until $46.7 \%$. Our results show that stratification reduces the $C V$ only in mixed forest and increases $R^{2}$ only in conifer forest. Other studies that applied stratification when combining remote sensing and field sampling found similar results than in our study, in $W$ estimation. Rodríguez-Veiga et al. (2016), using MNFI and MaxEnt algorithm, they fit a model to estimate $W$ at national-level with an $R^{2}$ of 0.31 , and found a decrease of $R^{2}$ at state-level with a range of 0.34 to 0.82 . Zhao et al. (2016) found that $R M S E$ and $C V$ decrease in the stratifications, except for the pine forest as in our study. In $180 \mathrm{~km}^{2}$ of a conifer forest of Turkey, Günlü et al. (2014) estimated $R^{2}$ of 0.61 and $C V$ of $10.19 \%$ for $W$ estimation. In a similar area of conifer forest in Turkey, Günlü \& Kadığulları (2018) estimated an $R^{2}$ of 41 and $C V$ of $8.9 \%$. Similar results for deciduous forest were obtained by Zhao et al. (2016) in estimates of AGB with $R^{2}$ of 0.43 and $R M S E$ of $24.8 \mathrm{Mg} \mathrm{ha}^{-1}$.

\section{V.4.4 Predictor variables from Landsat imagery}

The widest predictor variable ( $P V)$ used in $G$ and $W$ estimation is the NDVI (Gizachew et al., 2016; Pérez Miranda et al., 2018). However, there is evidence from previous studies that NDVI has limited ability to estimate $G$ and $W$ when high concentrations are estimated (Shi \& Liu, 2017). Through stepwise regression, we selected the $P V s$ which optimize (smaller RMSE) $G$ and $W$ estimates compared to those estimated with NDVI.

With the information of the first MNFI (2004-2009), we did not find optimization to the use of $N D V I$ to estimate $W$ in the temperate forest. However, in the conifer and oak forest strata, the estimate of $W$ was optimized with the simple ratio $(S R)$ vegetation index. For the mixed forest stratum, model optimization was done by adding a $P V$ with texture information (Rd11HC -

Haralicks correlation in window $11 X 11$ of the red band-) to the variable NDVI. In the second period of the MNFI (2009-2014), as in the first period, the optimal model to estimate $W$ in the temperate forest used the NDVI. For conifer forest and mixed forest, the $W$ estimate was optimized with the $S R$ by adding a $P V$ with texture (Haralicks correlation in $3 X 3$ of NDVI for conifer forest and weighted mean in 11X11 of ARVI for mixed forest). Finally, the AGB estimate for the oak forest was optimized with the SWIR2 band and the MSAVI index.

Applying GLCM-based texture and vegetation indexes different from NDVI, we optimized the $W$ (D. Lu, 2005)(D. Lu, 2005) estimated with NDVI, using different criteria. The models applied by Ou et al. (2019) selected the textures correlation, variance and dissimilarity applied to spectral bands in windows from $3 \mathrm{X} 3$ to $7 \mathrm{X} 7$ as the optimal $P V$ to estimate $W$ in conifer forest with Landsat. In the Amazon forest, Lu \& Batistella (2005) found that texture variance and dissimilarity in the NIR band optimizes $W$ estimates with Landsat 5. Sarker \& Nichol (2011) used textures contrast, skewness and second-moment angularity to improve estimates with ALOS AVNIR-2 imagery. In a boreal forest, Fuchs et al. (2009) reported that the AGB was optimized by using a 25X25 window in texture contrast for Quickbird and 5X5 for Aster, using the panchromatic layer. The improvement 
of the estimation with textures has been related to changes in forest structure ( $\mathrm{Lu}, 2005$; Sarker \& Nichol, 2011), as well as the complexity of crown structure (Ou et al., 2019); an important characteristic of the temperate forest of Durango (Aguirre et al., 2003; CONAFOR, 2014a; Márquez Linares et al., 2016; Návar \& González Elizondo, 2009).

In the models fitted without texture, the variables $S W I R 2, S R$, and MSAVI were used, since they have been shown to improve $W$ estimates. Cartus et al. (2014) found a high sensitivity form $S W I R$ bands to estimate $W$ at the national level in Mexico, and Molinier et al. (2016) optimized the estimate of $W$ using a model with SWIR2 and Green bands in Durango temperate forest. About $S R$, Jackson \& Huete (1991) found that it has a higher sensitivity for determining the amount and condition of vegetation with less influence of atmospheric conditions, compared to NDVI. Similarly, Qi et al. (1994) proposed the use of MSAVI to improve vegetation detection by decreasing the variations emitted by the soil. With the above, Zheng et al. (2004) estimated $W$ in a temperate forest using $M S A V I$ in their model together with $S R$ and tree age.

For the $G$ estimation with Landsat imagery, in the first period of the MNFI (2004-2009), the same $P V s$ were used as in the estimation of $W$. The only difference being that in the temperate forest there was optimization in the estimation of $G$, adding a texture $P V$ to the variable NDVI. In the second period of the MNFI (2009-2014), the estimation of $G$ was optimized in the temperate forest, and the strata mixed forest and oak forest, using texture information. In the case of the conifer forest stratum, the estimate was optimized using $S R$ instead of NDVI. The similarity in the adjusted models between $W$ and $G$ is assumed to be due to the close relationship in the variability of the estimates of $W$ and $G$ (correlation greater than 0.95). This similarity in the variation of $W$ and $G$ has also been reported in the tropical deciduous forest by Rao \& Rao (2015) with a correlation greater than 0.93 , and in temperate forest by Pérez Miranda et al. (2018), finding what they call "similar spatial variability".

The spatial distributions of variables $\mathrm{G}$ and $W$ are important in the planning and sustainable management of forest resources, to propose projects for the sustainable use of forest resources and/or environmental services such as carbon sequestration. Given the average estimate per vegetation type, together with analyses of land-use change, it is possible to detect which areas have shown the greatest loss in forest cover and to propose environmental policies for the optimization of forest resources, promoting the conservation of biodiversity and forest culture. Similarly, in areas with above-average estimates, intensive management can be proposed, applying sustainable strategies and thereby fixing carbon in wood for construction materials or furniture.

\section{V.4.5 Underestimation of the prediction}

The Bias was negative for all estimates, indicating an underestimation of the fitted models. Figure VIII-6 (Appendix VII) illustrates the $W$ underestimation of MNFI estimates by the predictor variables derived from Landsat. In the temperate forest (MNFI 2004-2009) and Landsat 5, $457 \mathrm{Mg}$ $\mathrm{ha}^{-1}$ was the maximum $W$ estimation observed, while the maximum $W$ estimated by the model was $154 \mathrm{Mg} \mathrm{ha}^{-1}$. In the same period at the strata-level, the maximum values of $W$ estimated were 152 , 164 and $75 \mathrm{Mg} \mathrm{ha}^{-1}$ for conifer, mixed and oak forests, respectively. Using Landsat 8 in the second period of MNFI, the maximum $W$ estimated were $165,156,169$ and $89 \mathrm{Mg} \mathrm{ha}^{-1}$, for temperate, conifer, mixed and oak forest. These results indicate a maximum limit of $W$ estimation from the predictor variables, and according to Ou et al. (2019), they represent the saturation in the $W$ 
estimation from Landsat imagery. Our results are similar to the saturation results obtained by Zhao et al. (2016), using Landsat 5 imagery they estimated 156, 159, 152 and $123 \mathrm{Mg} \mathrm{ha}^{-1}$, respectively to the same vegetation types. However, our results are above the saturation threshold of $70 \mathrm{Mg} \mathrm{ha}^{-1}$, proposed by Rodríguez-Veiga et al. (2017) for passive optical sensors. It was also observed that Landsat 8 increased the upper limit of $W$ estimation, compared to Landsat 5, Ou et al. (2019) observed this same in a temperate forest of China, reaching estimations greater than $180 \mathrm{Mg} \mathrm{ha}^{-1}$. The estimation of $G$ had similar behavior to $W$; the estimation shows saturation using the prediction variables of the Landsat imagery (Figure VIII-4, Appendix VII).

The underestimation ( $G$ and $W$ ) in this thesis, explains why by incorporating the spatial variability of Landsat images, the values obtained by the sample-based method decreased in a range of 9 and $13 \%$, for the first (2004-2009) and second (2009-2014) periods of the MNFI. In the estimation of $W$ at the national level, Rodríguez-Veiga et al. (2016) also obtained an underestimate of $W$ values by 2.4\% using MODIS and ALOS PALSAR imagery compared to the MNFI information. Muñoz-Ruiz et al. (2014), found underestimates also of $9 \%$ in Hidalgo (Mexico) for $G$ and wood volume. Fuchs et al. (2009), estimating $W$ in Siberian forest tundra with systematic sampling, underestimated $W$ values by $7 \%$ with Aster imagery; however, with Quickbird images it was overestimated by $1.5 \%$.

Figures VIII-5 and VIII-7 (Appendix VII) show the increase of the residuals with the size of the tree, despite the transformation made to the prediction variables to stabilize the variance. The heteroscedasticity is common in biological data such as $W$ estimations (Picard et al., 2012). It has been found in tundra forest using ASTER and Quickbird imagery (Fuchs et al., 2009), as well as with LIDAR and Landsat in a temperate forest (Lopez-Serrano et al., 2015; Ortiz-Reyes et al., 2015; Vargas-Larreta et al., 2017).

\section{V.4.6 Model-based comparison with sampling-based estimations}

The estimated variables ( $G$ and $W$ ) with model-based showed a higher precision due to a lower $S E$ estimated (Table IV-24). Therefore, a lower model-based $C_{95 \%}$ was estimated, as shown in Figure IV-15, while the sampling-based $C_{95 \%}$ was more conservative. Pérez Miranda et al. (2018) also estimated a lower $C I_{95 \%}$ in $G$ and $W$ using Landsat compared to MNFI in three municipalities of Durango. Similar results were obtained by Muñoz-Ruiz et al. (2014) in Hidalgo (Mexico) estimating a lower $I C_{95 \%}$ in $G$ and wood volume with SPOT compared with sampling-based estimation. Gizachew et al. (2016) also estimated lower $S E$ in $W$ estimates with Landsat compared to sampling-based estimation, in miombo woodlands in Tanzania.

In this thesis, sampling-based and model-based estimates were compared by calculating relative efficiency $(R E)$. This $R E$ was greater than 2.2 in the estimate of $G$ in temperate forest and greater than 4.7 in the estimate of $W$. In the temperate forest strata, the range was from 1.5 to 3.3 for $G$, while for $W$ it was from 2.3 to 6.4. The $R E$ represents the number of times the variance in the estimate ( $G$ and $W$ ) is reduced using Landsat imagery without additional costs since it is considered that the images were acquired without cost (González-Alonso et al., 1997). A $R E$ equal to 1.5 indicates the result of estimating $G$ with Landsat imagery in Durango is equivalent to increasing the sample size by 50\% (Næsset et al., 2016). Similar results were obtained by Hansen et al. (2015) in the rainforest, where they estimated $R E$ between 3.5 and 6.0 for 700 to $1900 \mathrm{~m}^{2}$ plots. In agriculture, higher values of $R E$ larger than 9.2 have been estimated because the agricultural areas show uniformity in shape and in the emission of NDVI (González-Alonso et al., 1997). 
Basal area $(G)$ and AGB $(W)$ maps were generated for the temperate forest in Durango, Mexico. These maps add the spatial variation, of Landsat satellite imagery (Sensor 5 -2007- and Sensor 8 2013-), to the sample-based estimates of the MNFI in Durango (2004-2009 and 2009-2014, respectively). These results are considered relevant because they allow us to know the spatial distribution, for the state of Durango, of the two estimated variables. The results make it possible to have precise and well-timed information (estimates) to monitor the dynamics and capture of carbon in this state, which is part of the MRV systems under REDD+ and is an express request in the estimation protocol of the Mexican Network of Carbon Intensive Monitoring Sites (Red MexSMIC, 2015). To optimize the results obtained in this thesis, in further steps we will include other remote sensors sources and thus reduce the saturation in the $W$ estimates. Different studies report optimizations of $W$ estimates using ALS (Hansen et al., 2015; Næsset et al., 2016), Radar (Sinha et al., 2015; Zhang et al., 2014; Zhao et al., 2016) and/or biophysical (Rodríguez-Veiga et al., 2016; Zhao et al., 2016) or bioclimatic characteristics (Lopez-Serrano et al., 2015). Similarly, we propose for a further step a different technique of regression models as machine learning (P. M. LópezSerrano et al., 2019; Rodríguez-Veiga et al., 2016; Vargas-Larreta et al., 2017) or mixed models (Nath et al., 2019; Wang et al., 2019) to improve the $W$ estimation in Durango temperate forest. 



\section{Chapter VI: Conclusions}

It was found that even with clear measurement protocols, instrument calibration, training, and nontime pressure measurements, random errors are present in the measurement of $\mathrm{DBH}$ and $\mathrm{TH}$ variables; and we can not avoid them (only less than $8 \%$ of re-measurements had no error). For this reason, the results concerning the comparison with reference or control data (commonly reported in scientific studies), the reference data should indicate the size $(\hat{\sigma})$ of the measurement errors.

With the technique of re-measurements, it was an efficient way to estimate the measurement errors. Re-measurements can describe the measurement errors using the mean value of the entire database or describe the errors by $D B H$ and $T H$ classes (heteroscedasticity). This is recommended to verify the size $(\hat{\sigma})$ of the errors in the forest estimates. The methods recommended describing the measurement errors of the measured variables were those including the heteroscedasticity of the measurement, i.e., the errors were estimated according to the size of $D B H$ and $T H$. In the temperate forest of Durango, Mexico, the best method using double measurement was the best distribution fitted by $D B H$ and TH class (BDC). In the temperate forest of Göttingen, Germany, the best method using five re-measurement was the linear model fitted with the tree variable size ( $\mathrm{mod}$ ).

It is important to emphasize the differences found in $T H$ measurements. With the data analyzed, we found that measurements made with Vertex had higher precision compared with the Blume-Leiss measurements. In this sense, it is recommended to use Vertex to improve the precision in $\mathrm{TH}$ measurement and the estimations made with this measurement (volume, AGB, dominance, etc.). However, the difference in measurement of $\mathrm{DBH}$, with Caliper and diametric Tape, are considered negligent due to no statistical difference was found.

The GUM Method of error propagation is explicit to generate knowledge, from the input of the measurement errors until the error estimation at the stand-level. Through the application of this method, error propagation was decomposed into sources and processes, and it is better understood how uncertainties are combined. The use of this method emphasizes the importance of the aggregation (during the sampling process) in the reduction of the non-sampling uncertainty. This reduction of uncertainty takes place when aggregating the AGB uncertainty from tree-level to plotlevel, and from plot-level to stand-level.

In measurement errors at tree-level, important contributions were found. The allometric models used in this thesis, which used $D B H$ and $T H$, have a sensitivity coefficients ratio $\left(S C_{D B H} / S C_{T H}\right)$ greater than one; this indicates that a greater contribution from the variable $D B H$ exists. However, it was also found that the ratio of the measurement errors in $D B H$ and $T H\left(\hat{\sigma}_{\mathrm{TH}} / \hat{\sigma}_{\mathrm{DBH}}\right)$ is decisive for further contribution in error propagation. If $\hat{\sigma}_{\mathrm{TH}} / \hat{\sigma}_{\mathrm{DBH}}$ is bigger than $S C_{D B H} / S C_{T H}$, then, the errors in $\mathrm{TH}$ measurement have a greater contribution than errors in $\mathrm{DBH}$ measurement at the tree-level. As is the case in the empirical studies made in this thesis.

Allometric models represent an area of opportunity in the estimation of AGB in Mexico. They are a challenge to conduct research using a greater number of trees in their fit, thus increasing their precision. A major challenge is to include in the publications of AGB allometric models, the Goodness-of-fit or even better, the access to the dataset used to construct the model.

Simultaneously, the training of the field crews of MNFI should be reinforced, with the purpose of achieving better identification of species and vegetation to apply the proper allometric model. 
At the plot-level, a relationship was found between the sources of the non-sampling uncertainty $(u N S)$, i.e. the uncertainty of the allometric models $(u A M)$ and the measurement errors (uMes). If the main contribution comes from $u A M$, then the $u N S$ estimate is in proportion to the number of trees. However, a relation $u M e s>u A M$ generates $u N S$ estimation proportional to the AGB estimate, which is related to the tree size.

The Monte-Carlo simulation Method $(M C M)$ prove to be an effective and practical way to estimate the propagation of errors in AGB estimation. It is easy to implement in personal computer equipment through the R packet. By applying this technique allows us to approximate the AGB estimate with acceptable ranges of probable error, and therefore reliable. The error propagation method used in this study is easy to implement in the NFI scale of work.

The results obtained in the error propagation by the GUM Method and by the MCM are equivalent. This occurs when measurement errors are estimated including the heteroscedasticity concerning to the size of the measured variable (size of the tree). Specifically, when employing normal distribution by classes in the GUM Method and best PDF by classes in MCM, the estimation by each source of uncertainty is compatible.

The largest contribution by these two methods of error propagation was $S E$, with over $98 \%$ of the total uncertainty in AGB estimation. Thus, the $S E$ is the largest source of uncertainty in the AGB estimation of Durango temperate forest. The second important source of error is the allometric model. This indicates that the errors made in $D B H$ and $T H$ measurements, as well as the uncertainty of the plot design, are negligible. However, the measurement errors should be part of the final report ( $M R V$ report) to reinforce the transparency and precision of the AGB estimation.

In the strata of temperate forest, AGB and basal area models fitted with texture information show the better prediction. Our study shows that regression models using texture improve the AGB estimation in conifer and mixed forest, in our study area. However, in the oak forest, no optimal results were obtained and need to be developed an appropriate method that can be applied to this forest type. Because the Landsat data is available on sub-annual timescales, the texture may be an important tool to optimize and update biomass maps with the partial's measurements of the annual MNFI surveys, or according to the phenology of the forest type. This will increase our understanding of the interaction of forest AGB and human activities (with low cost and proved efficiency), illustrating possible policy decisions in forest management.

We found an underestimation of the predicted values, related to the saturation of Landsat imagery in the AGB and basal area estimation. Although optimization of the estimates was achieved by including texture in the prediction model (the relative efficiency was positive), saturation in the estimates was not avoided. To improve the estimates, the use of RADAR imagery is proposed to include vegetation height as a required variable in the AGB estimation, due to its relationship to the tree volume.

This thesis provides the methodology to combine fieldwork and remote sensing data to improve the prediction of AGB and basal area in the Durango temperate forest. The methodology can be entirely replicated in Mexico because the materials used are public and are available (under request) for data processing. 
The models and maps developed show a high relationship between the AGB and the basal area, as previous studies in allometry are shown. Therefore, the basal area as an easy calculate variable, can be used as a proxy variable in the AGB estimation using remote sensing. Besides, the maps generated are a source of information for other disciplines to interact with, to develop more specific information like fuel accumulation, potential $\mathrm{CO}_{2}$ emissions in fire events, dynamics of carbon stock changes over time, among others. 



\section{Chapter VII: References}

Acosta Mireles, M., Carrillo Anzures, F., \& Gómez Villegas, R. (2011). Estimation of biomass and carbon in two mountain mesophilic forest species. Revista Mexicana de Ciencias Agricolas, 2(4), 529-543.

Acosta Mireles, M., Vargas Hernández, J., Velázquez Martínez, A., \& Etchevers Barra, J. D. (2002). Aboveground biomass estimation by means of allometric relationships in six hardwood species in Oaxaca, Mexico. Agrociencia, 36(06), 725-736.

Alberdi, I., Michalak, R., Fischer, C., Gasparini, P., Brändli, U. B., Tomter, S. M., ... Vidal, C. (2016). Towards harmonized assessment of European forest availability for wood supply in Europe. Forest Policy and Economics, 70(September), 20-29. https://doi.org/10.1016/j.forpol.2016.05.014

Alboabidallah, A., Martin, J., Lavender, S., \& Abbott, V. (2017). Using Landsat-8 and Sentinel-1 data for Above Ground Biomass assessment in the Tamar valley and Dartmoor. 2017 9th International Workshop on the Analysis of Multitemporal Remote Sensing Images (MultiTemp), 1-7.

Ascough, J. C., Maier, H. R., Ravalico, J. K., \& Strudley, M. W. (2008). Future research challenges for incorporation of uncertainty in environmental and ecological decision-making. Ecological Modelling, 219(3-4), 383-399. https://doi.org/10.1016/j.ecolmodel.2008.07.015

Attarchi, S., \& Gloaguen, R. (2014). Improving the estimation of above ground biomass using dual polarimetric PALSAR and ETM+ data in the Hyrcanian mountain forest (Iran). Remote Sensing, 6(5), 3693-3715. https://doi.org/10.3390/rs6053693

Avendaño Hernandez, D. M., Acosta Mireles, M., Carrillo Anzures, F., \& Etchevers Barra, J. D. (2009). Biomass and carbon estimation in an Abies religiosa forest. Revista Fitotecnia Mexicana, 32(3), 233238.

Ayala Gallego, G. (2015). Basic statistics. (S. Hocevar, Ed.) (2nd ed.). Sam Hocevar.

Azofeifa, C. E. (2005). Application of Monte Carlo Simulation in the calculation of risk using Excel. Tecnología En Marcha, 17(1), 97-109.

Bannari, A., Morin, D., Bonn, F., \& Huete, A. R. (1995). A review of vegetation indices. Remote Sensing Reviews, 13(1), 95-120. https://doi.org/10.1080/02757259509532298

Bartelink, H. H. (1996). Allometric relationships on biomass and needle area of Douglas-fir. Forest Ecology and Management, 86(1-3), 193-203. https://doi.org/10.1016/S0378-1127(96)03783-8

Basil, M., Papadopoulos, C., Sutherland, D., \& Yeung, H. (2001). Application of probabilistic uncertainty methods ( Monte- Carlo Simulation ) in flow measurement uncertainty. In Ø. Isaksen (Ed.), 19th International North Sea flow measurement workshop 2001 (pp. 1-21). Kristiansand, Norway: North sea flow measurement workshop.

Bechtold, W. A., \& Zarnoch, S. J. (1999). Field Methods And Data Processing Techniques Associated With Mapped Inventory Plots. In C. Aguirre-Bravo \& C. Rodriguez Franco (Eds.), The North American Science Symposium:Toward a Unified. Framework for Inventorying and Monitoring Forest Ecosystem Resources (pp. 421-424). Guadalajara, Jalisco, Mexico: USDA Forest Service.

Berger, A., Gschwantner, T., McRoberts, R. E., \& Schadauer, K. (2014). Effects of measurement errors on individual tree stem volume estimates for the Austrian national forest inventory. Forest Science, 60(1), $14-24$.

Bi, H. (2000). Trigonometric variable-form taper equations for Australian eucalypts. Forest Science, 46(3), 397-409.

Box, G. E. P., \& Cox, D. R. (1964). An Analysis of Transformations. Royal Statistical Society, 26(2), 211252.

Bramhe, V. S., Ghosh, S. K., \& Garg, P. K. (2018). Extraction of Built-Up Area By Combining Textural Features and Spectral Indices From Landsat-8 Multispectral Image. ISPRS - International Archives of the Photogrammetry, Remote Sensing and Spatial Information Sciences, XLII-5(November), 727-733. https://doi.org/10.5194/isprs-archives-xlii-5-727-2018

Brancalion, P. H. S., Campoe, O., Mendes, J. C. T., Noel, C., Moreira, G. G., van Melis, J., ... Guillemot, J. (2019). Intensive silviculture enhances biomass accumulation and tree diversity recovery in tropical forest restoration. Ecological Applications, 29(2), 1-12. https://doi.org/10.1002/eap.1847

Brickell, J. E. (1970). More on diameter tapes and calipers. Journal of Forestry, 68(3), 169-170.

Brown, S. (1997). Estimating biomass and biomass change of tropical forests: a primer. FAO Forestry Paper, 134(August), 55. Retrieved from http://www.fao.org/3/w4095e/w4095e00.htm

Brumme, R., \& Khanna, P. K. (2009). Functioning and management of european beech ecosystems. (M. M. Caldwell, G. Heldmaier, R. . Jackson, O. L. Lange, H. A. Mooney, E. D. Schulze, \& U. Sommer, Eds.), Ecological studies Vol. 208 (1st ed.). Heidelberg, Germany: Springer. 
BUN-CA. (2002). Biomass. Manuals on renewable energy. Strengthening Renewable Energy Capacity for Central America. San José, Costa Rica: Biomass Users Network (BUN-CA).

Búrquez, A., Martínez-Yrízar, A., Núñez, S., Quintero, T., \& Aparicio, A. (2010). Aboveground biomass in three Sonoran Desert communities: Variability within and among sites using replicated plot harvesting. Journal of Arid Environments, 74(10), 1240-1247.

Cairns, M. A., Olmsted, I., Granados, J., \& Argaez, J. (2003). Composition and aboveground tree biomass of a dry semi-evergreen forest on Mexico's Yucatan Peninsula. Forest Ecology and Management, 186(13), 125-132.

Canavan, S. J., \& Hann, D. W. (2014). Stochasticity and measurement error in forest models. Oregon, US. Retrieved from https://www.researchgate.net/publication/269699584_Stochasticity_and_Measurement_Error_in_Forest Models

Cano Santana, Z. (1994). Energy flow through sphenarium purpurascens (Orthoptera: acrididae) and net primary aerial productivity in a xerophytic community. National Autonomous University of Mexico (UNAM).

Cartus, O., Kellndorfer, J., Walker, W., Franco, C., Bishop, J., Santos, L., \& Fuentes, J. M. M. (2014). A national, detailed map of forest aboveground carbon stocks in Mexico. Remote Sensing, 6(6), 55595588. https://doi.org/10.3390/rs6065559

Castañuela Ramos, Y. E. (2013). Estimation of the Aerial Biomass and Carbon Capture in Yucca filifera (Chaubad) and Atriplex canescens (Pursh) Nutt. Using Allometric Equations, in Mazapíl, Zacatecas. Universidad Aiutónoma Agraria Antonio Narro.

Castro Quilantán, J. L., Bouchot, C., \& Sánchez Ochoa, J. C. (2010). Propagation of uncertainties, an evolutionary approach. In CENAM (Ed.), Simposio de Metrología 2010 (pp. 1-5). Queretaro, Qro., Mexico: Centro Nacional de Metrología (CENAM).

Chamber of Deputies. General Law of Sustainable Forestry Development (2018). Mexico.

Chamber of Deputies. General Law on climate change (2018). City of Mexico, Mexico, Mexico: Official Journal of the Federation (DOF).

Chamber of Deputies. Climate change: the main adaptation and mitigation actions in Mexico (2019). Mexico City, Mexico, Mexico.

Chave, J., Condit, R., Aguilar, S., Hernandez, A., Lao, S., \& Perez, R. (2004). Error propagation and scaling for tropical forest biomass estimates. Philosophical Transactions of the Royal Society of London. Series B, Biological Sciences, 359(1443), 409-420.

Chojnacky, D. C., Heath, L. S., \& Jenkins, J. C. (2014). Updated generalized biomass equations for North American tree species. Forestry, 87, 129-151. https://doi.org/10.1093/forestry/cpt053

CICC. (2013). National Climate Change Strategy. Vision 10-20-40. Mexico City, Mexico.

Cochran, W. G. (1977). Sampling Techniques. Technometrics (3rd.). New York, US: John Wiley and Sons.

CONABIO. (2017). Biodiversity in Durango. (A. Cruz Angón, E. Castaños Rochell, J. Valero Padilla, \& E. D. Melgarejo, Eds.). Mexico: National Commission for the Knowledge and Use of Biodiversity (CONABIO).

CONAFOR. (2009a). Anexo 1. Números del INFyS 2004-2009 (Annex 1. NFSI 2004-2009 numbers). National Forest and Soil Inventory 2004 - 2009. Zapopan, Jalisco, Mexico: National Forest Commission (CONAFOR).

CONAFOR. (2009b). Manual and procedures for field sampling. National Forest and Soil inventory 2004 2009. Zapopan, Jalisco, Mexico: National Forest Commission (CONAFOR).

CONAFOR. (2012a). Forests, climate change and REDD+ in Mexico. Basic Guide (First). (National Forest Commission (CONAFOR), Ed.). Zapopan, Jalisco, Mexico: National Forest Commission (CONAFOR).

CONAFOR. (2012b). Inventario Nacional Forestal y de Suelos, México 2004-2009. Zapopan, Jalisco, México.

CONAFOR. (2012c). National Forest and Soil Inventory of Mexico. Report 2004-2009. CONAFOR (Vol. 1). Zapopan, Jalisco, Mexico: National Forest Commission (CONAFOR). https://doi.org/10.1017/CBO9781107415324.004

CONAFOR. (2014a). Forest and soil inventory - Durango 2013. Tlalpan, Mexico.

CONAFOR. (2014b). Proposal of the reference level of the forest emissions of Mexico. Jalisco, Mexico.

CONAFOR. (2015). Practical guide on how to calculating state emission inventories of greenhouse gas. (National Forestry Commission (CONAFOR) \& Reinforcing REDD+ and South-South Cooperation, Eds.). Zapopan, Jalisco, Mexico: National Forestry Commission (CONAFOR), Reinforcing REDD+ and South-South Cooperationy Cooperación Sur-Sur. 
CONAFOR. (2017a). National Forest and Soil Inventory in Mexico Report 2009-2014. Memorias de la VII Reunión Nacional de Estadística. Zapopan, Jalisco, Mexico.

CONAFOR. (2017b). National strategy for REDD+ 2017-2030 (First). Zapopan, Jalisco, Mexico: National Forestry Commission (CONAFOR), Ministry of the Environment and Natural Resources (SEMARNAT).

Condit, R. (1998). Tropical forest census plots: Methods and Results from Barro Colorado Island, Panama and a Comparison with Other Plots. Georgetown, Texas, USA: Springer International Publishing. https://doi.org/10.1007/978-3-662-03664-8

Corral-Rivas, J. J., Márquez, P., Quiñones-Scott, R., Lujan-Soto, J. E., \& López-Sánchez, C. A. (2013). Installation of a thinning experiment in forests of UMAFOR 1008. In A. R. Ortíz Gámez \& J. Méndez González (Eds.), XI Mexican Congress on Forest Resources 2013 (pp. 433-442). Saltillo, Coahuila, Mexico: Mexican Forest Resources Society and Antonio Narro Autonomous Agrarian University.

Corral-Rivas, José Javier, Vargas Larreta, B., Wehenkel, C., Aguirre Calderón, O. A., Álvarez González, J. G., \& Rojo Alboreca, A. (2009). Guide for the establishment of forestry and soil research in forests of the state of Durango (p. 54). Durango, Mexico.

Criscenti, L. J., Laniak, G. F., \& Erikson, R. L. (1996). Propagation of uncertainty through geochemical code calculations. Geochimica et Cosmochimica Acta, 60(19), 3551-3568. https://doi.org/10.1016/00167037(96)00188-3

Diéguez Aranda, U., Castedo Dorado, F., Barrio Anta, M., Álvarez González, J. G., Rojo Alboreca, A., \& Ruiz González, A. D. (2005). Forest mensuration practices. (Fundación Conde del Valle de Salazar y Ediciones Mundi-Prensa, Ed.) (1st ed.). Lugo, España: Universidad de Santiago de Compostela.

Dohoo, I. R., Ducrot, C., Fourichon, C., Donald, A., \& Hurnik, D. (1997). An overview of techniques for dealing with large numbers of independent variables in pidemiologic studies. Preventive Veterinary Medicine, 29(3), 221-239. https://doi.org/https://doi.org/10.1016/S0167-5877(96)01074-4

Draper, N. R., \& Smith, H. (1998). Applied Regression Analysis. (V. Barnett, R. A. Bradley, N. A. C. Cressie, N. I. Fisher, lain M. Johnstone, J. B. Kadane, ... E. J. Stuart Hunter, Eds.) (3rd ed.). New York, Chchester, Weinheim, Brisbane, Singapore, Toronto: John Wiley \& Sons, Inc.

Ducey, M. J., Zarin, D. J., Vasconcelos, S. S., \& Araújo, M. M. (2009). Biomass equations for forest regrowth in the eastern Amazon using randomized branch sampling. Acta Amazonica, 39(2), 349-360.

Elzinga, C., Shearer, R. C., \& Elzinga, G. (2005). Observer variation in tree diameter measurements. Western Journal of Applied Forestry, 20(2), 134-137.

Fahrmeir, L., Kneib, T., Lang, S., \& Marx, B. (2013). Regression. Models, methods and application (1st ed.). Heidelberg, New York, Dordrecht, London: Springer.

FAO. (1981). Manual of forest inventory. Rome, Italy. Retrieved from http://www.fao.org/3/ap358e/ap358e00.pdf

FAO. (2006). Global Forest Resources Assessment 2005. Rome, Italy. https://doi.org/ISBN 92-5-105481-9

FAO. (2015). Global Forest Resources Assessment 2015. Rome, Italy. Retrieved from http://www.fao.org/forestry/fra2005/en/

Farrance, I., \& Frenkel, R. (2014). Uncertainty in measurement: A review of monte carlo simulation using microsoft excel for the calculation of uncertainties through functional relationships, including uncertainties in empirically derived constants. Clinical Biochemist Reviews, 35(1), 37-61. https://doi.org/http://dx.doi.org/10.1016/j.probengmech.2016.04.005

Fehrmann, L. (2006). Alternative approaches for biomass estimation on single tree level with $k$-nearest neighbor method. University of Göttingen, Göttingen, Germany.

Foroughbakhch, R., Alvarado-Vázquez, M. A., Hernández-Piñero, J. L., Rocha-Estrada, A., Guzmán-Lucio, M. A., \& Treviño-Garza, E. J. (2006). Establishment, growth and biomass production of 10 tree woody species introduced for reforestation and ecological restoration in northeastern Mexico. Forest Ecology and Management, 235(1-3), 194-201.

Fuchs, H., Magdon, P., Kleinn, C., \& Flessa, H. (2009). Estimating aboveground carbon in a catchment of the Siberian forest tundra: Combining satellite imagery and field inventory. Remote Sensing of Environment, 113(3), 518-531. https://doi.org/10.1016/j.rse.2008.07.017

García-Pérez, A. (2014). Data Interpretation. An introduction to applied statistic. (UNED, Ed.) (First). Madrid, Spain: National University of Distance Education - Universidad Nacional de Educación a Distancia (UNED)-.

Garzuglia, M. (2018). 1948-2018: Seventy years of FAO's Global Forest Resources Assessment. Historical overview and future prospects. (S. Russell \& D. Henderson-Howat, Eds.) (1st ed.). Rome, Italy: Forest and Agriculture Organization of the United Nations (FAO). 
Gaudin, S., \& Richard, J. B. (2014). Comparaison des dendromètres vertex III et trupulse 200b pour la mesure de la hauteur totale des arbres. Revue Forestiere Francaise, 66(2), 163-181. https://doi.org/10.4267/2042/54353

GAUG. (2017). Forstbotanischer Garten (Forest Botanical Garden). Retrieved August 22, 2017, from http://www.uni-goettingen.de/en/site-and-climate/10232.html

Gertner, G., \& Köhl, M. (1992). An assessment of some nonsampling errors in a national survey using an error budget. Forest Science, 38(3), 525-538.

GFOI. (2016). Integration of Remote-Sensing and Ground-Based Observations for Estimation of Emissions and Removals of Greenhouse Gases in Forests. Methods and Guidance from the Global Forest Observations Initiative. (GFOI, Ed.) (2nd ed.). Rome, Italy.

Gibbs, H. K., Brown, S., Niles, J. O., \& Foley, J. A. (2007). Monitoring and estimating tropical forest carbon stocks: Making REDD a reality. Environmental Research Letters, 2(4).

Gil, S., \& Rodríguez, E. (2001). Re-Creative Physics (1st ed.). Buenos Aires, Argentina: Prentice-Hall Hispananoamericana, S. A.

Gitelson, A. a. (2004). Wide Dynamic Range Vegetation Index for remote quantification of biophysical characteristics of vegetation. Journal of Plant Physiology, 161(2), 165-173. https://doi.org/10.1078/0176-1617-01176

Gizachew, B., Solberg, S., Næsset, E., Gobakken, T., Bollandsås, O. M., Breidenbach, J., ... Mauya, E. W. (2016). Mapping and estimating the total living biomass and carbon in low-biomass woodlands using Landsat 8 CDR data. Carbon Balance and Management, 11(1), 1-14.

Glenn, E. P., Huete, A. R., Nagler, P. L., \& Nelson, S. G. (2008). Relationship between remotely-sensed vegetation indices, canopy attributes and plant physiological processes: What vegetation indices can and cannot tell us about the landscape. Sensors, 8(4), 2136-2160. https://doi.org/10.3390/s8042136

Goliaš, M., \& Palenčár, R. (2012). Determination of uncertainties for correlated input quantities by the Monte Carlo method. Acta Polytechnica, 52(4), 57-61. https://doi.org/10.14311/1590

González-Alonso, F., Cuevas, J. M., Arbiol, R., \& Baulies, X. (1997). Remote sensing and agricultural statistics: crop area estimation in north-eastern Spain through diachronic Landsat TM and ground sample data. Remote Sensing, 18(2), 467-470.

González-Elizondo, M. S., González-Elizondo, M., Tena-Flores, J. A., Ruacho-González, L., \& LópezEnríquez, I. L. (2012). Vegetation of Sierra Madre Occidental, Mexico: A synthesis work. Acta Botanica Mexicana, 100, 351-403.

Gormanson, D. D., Pugh, S. A., Barnett, C. J., Miles, P. D., Morin, R. S., Sowers, P. A., \& Westfall, J. A. (2017). Statistics and Quality Assurance for the Northern Research Station Forest Inventory and Analysis Program, 2016. Newtown Square, PA, USA. https://doi.org/10.2737/NRS-GTR-166

GTOS, \& FAO. (2009). Biomass. Assessment of the status of the development of the standards for the rterrestrial essencial climate variables (Vol. 10). Rome, Italy.

Günlü, A., Ercanli, I., Başkent, E. Z., \& Çakır, G. (2014). Estimating aboveground biomass using Landsat TM imagery: A case study of Anatolian Crimean pine forests in Turkey. Annals of Forest Research, 57(2), 289-298. https://doi.org/10.15287/afr.2014.278

Günlü, Alkan, \& Kadıogulları, A. İ. (2018). Modeling forest stand attributes using landsat ETM+ and quickbird satellite images in western Turkey. Bosque, 39(1), 49-59. https://doi.org/10.4067/S071792002018000100049

Haglöf Sweden AB. (2007). Users Guide Vertex IV and Transponder T3. Långsele, Sweden: Haglöf Sweden AB.

Hansen, E. H., Gobakken, T., Solberg, S., Kangas, A., Ene, L., Mauya, E., \& Næsset, E. (2015). Relative efficiency of ALS and InSAR for biomass estimation in a Tanzanian rainforest. Remote Sensing, 7(8), 9865-9885. https://doi.org/10.3390/rs70809865

Haralick, R. M., Shanmugam, K., \& Dinstein, I. (1973). Textural Features for Image Classification. IEEE Transactions on Systems, Man, and Cybernetics, SMC-3(6), 610-621. https://doi.org/10.1109/tsmc.1973.4309314

Henebry, G., Viña, A., \& Gitelson, A. (2004). The wide dynamic range vegetation index and its potential utility for gap analysis. Papers in Natural Resources, 50-56. Retrieved from http://digitalcommons.unl.edu/natrespapers/262/

Hill, R. C., Griffiths, W. E., \& Lim, G. C. (2018). Principles of Econometrics (5th ed.). USA: Wiley John Wiles \& Sons, Inc.

Holdaway, R. J., McNeill, S. J., Mason, N. W. H., \& Carswell, F. E. (2014). Propagating uncertainty in plotbased estimates of forest carbon stock and carbon stock change. Ecosystems, 17(4), 627-640. 
https://doi.org/10.1007/s10021-014-9749-5

Houghton, R. A. (2005). Aboveground forest biomass and the global carbon balance. Global Change Biology, 11(6), 945-958. https://doi.org/10.1111/j.1365-2486.2005.00955.x

Hughes, I. G., \& Hase, T. P. A. (2010). Measurements and their uncertainties. A practical guide to modern error analysis (1st ed.). New York, USA: Oxford University Press.

Hyde, P., Dubayah, R., Walker, W., Blair, J. B., Hofton, M., \& Hunsaker, C. (2006). Mapping forest structure for wildlife habitat analysis using multi-sensor (LiDAR, SAR/InSAR, ETM+, Quickbird) synergy. Remote Sensing of Environment, 102(1-2), 63-73. https://doi.org/10.1016/j.rse.2006.01.021

Hyppönen, M., \& Roiko-Jokela., P. (1978). On the accuracy and effectivity of measuring sample trees. Folia Forestalia, 356, 1-25.

INEGI. (2014). Dictionary of Land Use and Vegetation Data. Scale 1: 250000. (INEGI, Ed.) (Third). Aguascalientes, Mexico: National Institute of Statistic and Geography (INEGI).

INEGI. (2016). Statistical and geographical yearbook of Durango 2016. (INEGI, Ed.) (1st ed.). Aguascalientes, Mexico: INEGI.

INEGI. (2017). Guide to the interpretation of cartography: land use and vegetation. (INEGI, Ed.), Scale 1:250,000: series VI. (First). Aguascalientes, Mexico: National Institute of Statistic and Geography (INEGI).

IPCC. (2003). Good practice guidance for land use, land use change and forestry. Institute for Global Environmental Strategies. Hayanama, Kanagawa, Japan: Intergovernmental Panel on Climate Change (IPCC).

IPCC. (2006). Volume 4: Agriculture, Forestry and Other Land Use. In S. Eggleston, L. Buendia, K. Miwa, T. Ngara, \& K. Tanabe (Eds.), 2006 IPCC Guidelines for National Greenhouse Gas Inventories (First, p. 673). Kanagawa, Japan: IPCC.

IPCC. (2013). Climate Change 2013: The Physical Science Basis. Contribution of Working Group I to the Fifth Assessment Report of the Intergovernmental Panel on Climate Change. (T. F. Stocker, D. Qin, G.K. Plattner, M. Tignor, S. K. Allen, J. Boschung, ... P. M. Midgley, Eds.) (First). Geneva, Switzerland: Cambridge University Press.

IPCC. (2014). Climate Change 2014: Synthesis Report. Contribution of Working Groups I, II and III to the Fifth Assessment Report of the Intergovernmental Panel on Climate Change. (Core Writting Team IPCC, R. K. Pachauri, \& L. Meyer, Eds.). Geneva, Switzerland: Intergovernmental Panel on Climate Change. https://doi.org/10.1017/CBO9781107415324

IPCC. (2015). Climate Change 2014. Mitigation of Climate Change. Summary for policymakers and Technical Summary. https://doi.org/10.1177/002248717302400108

Jackson, R., \& Huete, A. R. (1991). Interpreting vegetation indexes. Preventive Veterinary Medicine, 11, $185-200$.

James, G., Witten, D., Hastie, T., \& Tibshirani, R. (2013). An Introduction to Statistical Learning with applications in R. (G. Casella, S. Fienberg, \& I. Olkin, Eds.) (First). New York, Heidelberg, Dordrecht, London: Springer International Publishing. https://doi.org/10.1016/j.peva.2007.06.006

JCGM. (2010). Evaluation of measurement data: Guide to the expression of uncertainty in measurement. (I. and O. BIPM, IEC, IFCC, ILAC, ISO, IUPAC, Ed.) (2nd ed.). Joint Committee for Guides in Metrology (JCGM).

Kallner, A. (2001). Uncertainty in measurement, Introduction and examples from laboratory medicine. JIFCC , 13(1), 16-24. Retrieved from http://www.ifcc.org/ ejifcc/vol13no1/1301200103.htm

Kangas, A. S. (1998). Effect of errors-in-variables on coefficients of a growth model and on prediction of growth. Forest Ecology and Management, 102(2-3), 203-212. https://doi.org/10.1016/S03781127(97)00161-8

Kauffman, B. J., Donato, D., \& Adame, M. F. (2013). Protocol for the measurement, monitoring and reporting of mangrove structure, biomass and carbon stocks (No. 117). Documento de Trabajo 117. Bogor, Indonesia.

Kaufman, Y. J., \& Tanré, D. (1992). Atmospherically resistant vegetation index (ARVI) for EOS-MODIS. IEEE Transactions on Geoscience and Remote Sensing, 30(2), 261-270.

Kelsey, K. C., \& Neff, J. C. (2014). Estimates of aboveground biomass from texture analysis of landsat imagery. Remote Sensing, 6(7), 6407-6422. https://doi.org/10.3390/rs6076407

Kershaw Jr., J. A., Ducey, M. J., Beers, T. W., \& Husch, B. (2017). Forest mensuration. (L. John Wiley \& Sons, Ed.) (5th ed.). Chichester, West Sussex, UK: Wiley Blackwell.

Kitahara, F., Mizoue, N., \& Yoshida, S. (2010). Effects of training for inexperienced surveyors on data quality of tree diameter and height measurements. Silva Fennica, 44(4), 657-667. 
https://doi.org/10.14214/sf.133

Kleinn, C. (2017). The renaissance of National Forest Inventories (NFIs) in the context of the international conventions - a discussion paper on context, background and justification of NFIs. Pesquisa Florestal Brasileira, 37(91), 369. https://doi.org/10.4336/2017.pfb.37.91.1343

Kleinn, C., Bhandari, N., \& Fehrmann, L. (2015). Observations and measurements. In FAO (Ed.), Knowledge reference for national forest assessments (1st ed., pp. 41-52). Rome, Italy: Food and Agriculture Organization (FAO). Retrieved from http://www.fao.org/forestry/fma/73411/en/

Köhl, M., Magnussen, S. S., \& Marchetti, M. (2006). Sampling Methods, Remote Sensing and GIS Multiresource Forest Inventory, 392.

Langford, J. (2005). The Cross Validation Problem. In P. Auer \& R. Meir (Eds.), Learning Theory. COLT 2005. Lecture Notes in Computer Science, vol 3559 (pp. 687-688). Berlin, Heidelberg: Springer. https://doi.org/10.1007/11503415_47

Le Maire, G., François, C., \& Dufrêne, E. (2004). Towards universal broad leaf chlorophyll indices using PROSPECT simulated database and hyperspectral reflectance measurements. Remote Sensing of Environment, 89(1), 1-28. https://doi.org/10.1016/j.rse.2003.09.004

Lewis, S. L., Sonké, B., Sunderland, T., Begne, S. K., Lopez-Gonzalez, G., van der Heijden, G. M. F., ... Zemagho, L. (2013). Above-ground biomass and structure of 260 African tropical forests. Philosophical Transactions of the Royal Society B: Biological Sciences, 368(1625), 14. https://doi.org/10.1098/rstb.2012.0295

Liu, S., Bitterlich, W., Cieszewski, C. J., \& Zasada, M. J. (2011). Comparing the use of three dendrometers for measuring diameters at breast height. Southern Journal of Applied Forestry, 35(3), 136-141. https://doi.org/10.1093/sjaf/35.3.136

López-Serrano, P., López-Sánchez, C., Díaz-Varela, R., Corral-Rivas, J., Solís-Moreno, R., Vargas-Larreta, B., \& Álvarez-González, J. (2015). Estimating biomass of mixed and uneven-aged forests using spectral data and a hybrid model combining regression trees and linear models. IForest - Biogeosciences and Forestry, (October), e1-e9. https://doi.org/10.3832/ifor1504-008

López-Serrano, P. M., Cárdenas Domínguez, J. L., Corral-Rivas, J. J., Jiménez, E., López-Sánchez, C. A., \& Vega-Nieva, D. J. (2019). Modeling of Aboveground Biomass with Landsat 8 OLI and Machine Learning in Temperate Forests. Forests, 11(11), 1-18. https://doi.org/10.3390/f11010011

Lopez-Serrano, P. M., Corral-Rivas, J. J., \& López-Sánchez, C. A. (2015). Spatial mapping carbon stocks in temperate forests of the state of Durango, Mexico. In F. P. Pellat, J. W. González, \& R. T. Alamilla (Eds.), Current state of knowledge of the carbon cycle and its interactions in Mexico: synthesis 2015. National Synthesis Series (p. 702). Texcoco, Mexico: Mexican Carbon Program (PMC) in collaboration with the Center for Global Change and Sustainability in the Southeast, A.C. and the International Center for Linking and Teaching of the Universidad Juárez Autónoma de Tabasco.

Lu, D. (2005). Aboveground biomass estimation using Landsat TM data in the Brazilian Amazon.

International Journal of Remote Sensing, 26(12), 2509-2525.

https://doi.org/10.1080/01431160500142145

Lu, Dengsheng. (2006). The potential and challenge of remote sensing-based biomass estimation.

International Journal of Remote Sensing, 27(7), 1297-1328.

https://doi.org/10.1080/01431160500486732

Lu, Dengsheng, \& Batistella, M. (2005). Exploring TM image texture and its relationships with biomass estimation in Rondônia, Brazilian Amazon. Acta Amazonica, 35(2), 249-257. https://doi.org/10.1590/s0044-59672005000200015

Lund, H. G. (2009). National forest inventories and global resource assessments. In J. N. Owens \& H. G. Lund (Eds.), Forest and Forest Plants Volume 1, Encyclopedia of Life Support Systems (EOLSS) (First, pp. 29-65). Oxford, UK: EOLSS Publishers.

Luoma, V., Saarinen, N., Wulder, M. A., White, J. C., Vastaranta, M., Holopainen, M., \& Hyyppä, J. (2017). Assessing precision in conventional field measurements of individual tree attributes. Forests, 8(2), 116. https://doi.org/10.3390/f8020038

Ma, W., Domke, G. M., Woodall, C. W., \& D’Amato, A. W. (2019). Land use changes, disturbances, and their interactions on future forest aboveground biomass dynamics in the Northern US. Forests, 10(7), 121. https://doi.org/10.3390/f10070606

Magnussen, S., \& Reed, D. (2015). Modelling for estimation and monitoring. In FAO (Ed.), Knowledge reference for national forest assessments (First, pp. 111-136). Rome, Italy: Food and Agriculture Organization (FAO). Retrieved from http://www.fao.org/forestry/fma/73411/en/

Mahmoud, G. M., \& Hegazy, R. S. (2017). Comparison of GUM and Monte Carlo methods for the 
uncertainty estimation in hardness measurements. International Journal of Metrology and Quality Engineering, 8, 1-9. https://doi.org/10.1051/ijmqe/2017014

Marchau, V. A. W. J., Walker, W. E., Bloemen, P. J. T. M., \& Popper, W. S. (2019). Decision Making under Deep Uncertainty From Theory to Practice. (V. A. W. J. Marchau, W. E. Walker, P. J. T. M. Bloemen, \& W. S. Popper, Eds.) (First). Gelderland, Zuid-Holland,The Hague, The Netherlands; Santa Monica, CA, USA: Springer. https://doi.org/https://doi.org/10.1007/978-3-030-05252-2

Marquardt, D. W. (1970). Generalized inverses, ridge regression, biased linear estimation, and nonlinear estimation. American Statistical Association, American Society for Quality. Taylor \& Francis., 12(3), 591-612.

Marsett, R. C., Qi, J., Heilman, P., Biedenbender, S. H., Watson, M. C., Amer, S., ... Marsett, R. (2006). Remote Sensing for Grassland Management in the Arid Southwest. Rangeland Ecology \& Management, 59(5), 530-540. https://doi.org/10.2111/05-201R.1

Martinez-Yrizar, A., Sarukhan, J., Perez-Jimenez, A., Rincón, E., Maass, J. M., Solis-Magallanes, A., \& Cervantes, L. (1992). Above-ground phytomass of a tropical deciduous forest in the coast of Jalisco, México. Journal Tropical Ecology, 8(1), 87-96.

Martínez Barrón, R. A., Aguirre Calderón, O. A., Vargas Larreta, B., Jiménez Pérez, J., Treviño Garza, E. J., \& Yerena Yamallel, J. I. (2016). Modeling of biomass and aboveground carbon in forests of the state of Durango. Revista Mexicana de Ciencias Forestales, 7(35), 91-106.

Martínez, E. N. (2003). Calculating error bounds in accidentología using the Monte Carlo technique. Bariloche, Argentina.

Matérn, B. (1956). On the Geometry of the Cross-Section of a Stem. Stockholm.

McRoberts, R. E., Hahn, J. T., Hefty, G. J., \& Cleve, J. R. Van. (1994). Variation in forest inventory field measurements. Canadian Journal of Forest Research, 24(9), 1766-1770.

McRoberts, R. E., Næsset, E., \& Gobakken, T. (2015). Optimizing the k-Nearest neighbors technique for estimating forest aboveground biomass using airborne laser scanning data. Remote Sensing of Environment, 163(June), 13-22.

McRoberts, R. E., Tomppo, E. O., \& Czaplewski, R. L. (2015). Sampling designs for national forest assessments. In FAO (Ed.), Knowledge reference for national forest assessments (First, pp. 23-40). Rome, Italy: Food and Agriculture Organization (FAO). Retrieved from http://www.fao.org/forestry/fma/73411/en/

McRoberts, R. E., \& Westfall, J. A. (2014). Effects of uncertainty in model predictions of individual tree volume model on large area volume estimates. Forest Science, 60(1), 34-42.

McRoberts, R. E., \& Westfall, J. A. (2016). Propagating uncertainty through individual tree volume model predictions to large-area volume estimates. Annals of Forest Science, 73(3), 625-633.

Mehtätalo, L. (2013). Forest biometrics with examples in R. Kuopio, Finland: University of Eastern, Finland.

Melson, S., Azuma, D., \& Fried, J. S. (2001). A first look at measurement error on FIA plots using blind plots in the Pacific Northwest. In R. E. McRoberts, G. A. Reams, P. C. Van Deusen, \& J. W. Moser (Eds.), Thrid Annual Forest Inventory and Analysis Symposium (pp. 11-20). Saint Paul, Minnesota, US: U.S. Department of Agriculture, Forest Service, North Central Research Station.

Méndez González, J., Turlan Medina, O. A., Ríos Saucedo, J. C., \& Nájera Luna, J. A. (2012). Allometric equations to estimate aerial biomass of Prosopis laevigata (Humb. \& Bonpl. ex Willd.) M.C. Johnst. Rev. Mex. Cien. For. Vol., 3(13), 57-72.

Menéndez-Miguélez, M., Canga, E., Barrio-Anta, M., Majada, J., \& Álvarez-Álvarez, P. (2013). A three level system for estimating the biomass of Castanea sativa Mill. coppice stands in north-west Spain. Forest Ecology and Management, 291, 417-426. https://doi.org/10.1016/j.foreco.2012.11.040

Mexican Government. The National Development Plan of Mexico 2019-2024 (2019). Mexico City, Mexico, Mexico. Retrieved from https://lopezobrador.org.mx/wp-content/uploads/2019/05/PLAN-NACIONALDE-DESARROLLO-2019-2024.pdf

Molinier, M., López-Sánchez, C. A., Toivanen, T., Korpela, I., Corral-Rivas, J. J., Tergujeff, R., \& Häme, T. (2016). Relasphone-mobile and participative in situ forest biomass measurements supporting satellite image mapping. Remote Sensing, 8(869), 1-23. https://doi.org/10.3390/rs8100869

Morales M., E. H. (2005). Experimental design through the Analysis of Variance and Linear Regression Model. (A. Diaz M., Ed.) (First). Valdivia, Chile: Carouna.

Moran, L. A., \& Williams, R. A. (2002). Comparison of three dendrometers in measuring diameter at breast height. Northern Journal of Applied Forestry, 19(1), 28-33. https://doi.org/10.1093/njaf/19.1.28

Morfín Ríos, J. E., Michel Fuentes, J. M., González Murguía, R., Carrillo, O., Mayorga, R., Rangel, L., ... Guerrero Pacheco, G. (2015). Carbon reserves estimation in forest biomass in Mexico. Zapopan, 
Jalisco, Mexico.

Morrison, L. W. (2016). Observer error in vegetation surveys : a review. Plant Ecology, 9(4), 367-379. https://doi.org/10.1093/jpe/rtv077

Mostafa, S. A., \& Ahmad, I. A. (2018). Recent developments in systematic sampling: A review. Journal of Statistical Theory and Practice, 12(2), 290-310. https://doi.org/10.1080/15598608.2017.1353456

Muñoz-Ruiz, M. A., Valdez-Lazalde, J. R., de los Santos-Posadas, H. M., Ángeles-Pérez, G., \& MonterrosoRivas, A. I. (2014). Inventory and mapping of temperate forest in Hidalgo, Mexico through spot and field data. Agrociencia, 48(8), 847-862.

Næsset, E., Ørka, H. O., Solberg, S., Bollandsås, O. M., Hansen, E. H., Mauya, E., ... Gobakken, T. (2016). Mapping and estimating forest area and aboveground biomass in miombo woodlands in Tanzania using data from airborne laser scanning, TanDEM-X, RapidEye, and global forest maps: A comparison of estimated precision. Remote Sensing of Environment, 175, 282-300. https://doi.org/10.1016/j.rse.2016.01.006

Nagel, U., \& Wunderlich, H.-G. (1976). Geological block image of the surroundings of Göttingen. Lower Saxony Institute of Regional and Regional Development (2nd ed., Vol. 91). Göttingen, Germany.

Nath, A. J., Tiwari, B. K., Sileshi, G. W., Sahoo, U. K., Brahma, B., Deb, S., .. Gupta, A. (2019). Allometric models for estimation of forest biomass in North East India. Forests, 10(2). https://doi.org/10.3390/f10020103

Návar, J. (2009). Allometric equations for tree species and carbon stocks for forests of northwestern Mexico. Forest Ecology and Management, 257(2), 427-434.

Návar, J., Méndez, E., Nájera, A., Graciano, J., Dale, V., \& Parresol, B. (2004). Biomass equations for shrub species of Tamaulipan thornscrub of North-eastern Mexico. Journal of Arid Environments, 59(4), 657674.

Ni-Meister, W., Lee, S., Strahler, A. H., Woodcock, C. E., Schaaf, C., Yao, T., ... Blair, J. B. (2010). Assessing general relationships between aboveground biomass and vegetation structure parameters for improved carbon estimate from lidar remote sensing. Journal of Geophysical Research: Biogeosciences, 115(G2), n/a-n/a. https://doi.org/10.1029/2009jg000936

Ogilvie, J. F. (1984). A monte-carlo approach to error propagation. Computers and Chemistry, 8(3), $205-207$. https://doi.org/10.1016/0097-8485(84)80007-8

Ortiz-Reyes, A. D., Valdez-Lazalde, R. J., De Los Santos-Posadas, H. M., Ángeles-Pérez, G., Paz-Pellat, F., \& Martínez-Trinidad, T. (2015). Inventory and cartography of forest variables derived from LiDAR data: comparison of methods. Madera y Bosques, 21(3), 111-128.

Ou, G., Li, C., Lv, Y., Wei, A., Xiong, H., Xu, H., \& Wang, G. (2019). Improving aboveground biomass estimation of Pinus densata forests in Yunnan using Landsat 8 imagery by incorporating age dummy variable and method comparison. Remote Sensing, 11(7), 738. https://doi.org/10.3390/rs11070738

Pardé, J., \& Bouchon, J. (1988). Dendrometry (2nd\|). Nancy, France: National School of Rural Engineering, Water and Forestry.

PASCC. (2010). Advancing the Science of Climate Change. (Panel on advancing the science of climate change (PASCC) \& National Academy of Sciences (NAC), Eds.) (First). Washington, DC: National Academies Press.

Pérez-Cruzado, C., Fehrmann, L., Magdon, P., Cañellas, I., Sixto, H., \& Kleinn, C. (2015). On the site-level suitability of biomass models. Environmental Modelling and Software, 73, 14-26. https://doi.org/10.1016/j.envsoft.2015.07.019

Pérez-Hernández, M. M. (2012). Uncertainty estimation. GUM guide. E-Medida. Revista Española de Metrología, 1(3), 113-130.

Pérez Miranda, R., Romero Sánchez, M. E., González Hernández, A., Martínez Angel, L., \& Arriola Padilla, V. J. (2018). Estimation of forest attributes by remote sensing in mixed forests of Durango, Mexico. Áreas Naturales Protegidas Scripta, 4(2), 49-70. https://doi.org/10.18242/anpscripta.2018.04.04.02.0003

Peuhkurinen, J., Maltamo, M., \& Malinen, J. (2008). Estimating species-specific diameter distributions and saw log recoveries of boreal forests from airborne laser scanning data and aerial photographs: A distribution-based approach. Silva Fennica, 42(4), 625-641.

Phalla, T., Ota, T., Mizoue, N., Kajisa, T., Yoshida, S., Vuthy, M., \& Heng, S. (2018). The importance of tree height in estimating individual tree biomass while considering errors in measurements and allometric models. Agrivita, 40(1), 131-140. https://doi.org/10.17503/agrivita.v40i1.1730

Phillips, D. L., Brown, S. L., Schroeder, P. E., \& Birdsey, R. A. (2000). Toward error analysis of large-scale forest carbon budgets. Global Ecology and Biogeography, 9(4), 305-313. 
https://doi.org/10.1046/j.1365-2699.2000.00197.x

Phua, M.-H., Ling, Z.-Y., Wong, W., Korom, A., Ahmad, B., Besar, N. A., ... Takao, G. (2012). Estimating aboveground biomass of a tropical forest in Northern Borneo based on individual tree crowns from IKONOS 2 data. In 33rd Asian Conference on Remote Sensing (pp. 1-8).

Picard, N., Saint-André, L., \& Henry, M. (2012). Manual for building tree volume and biomass allometric equations: from field measurement to prediction. (FAO, Ed.), Cirad (First). Rome, Italy: CIRAD and Food and Agricultural Organization of the United Nations (FAO).

Pimple, U., Sitthi, A., Simonetti, D., Pungkul, S., Leadprathom, K., \& Chidthaisong, A. (2017). Topographic correction of Landsat TM-5 and Landsat OLI-8 imagery to improve the performance of forest classification in the mountainous terrain of Northeast Thailand. Sustainability (Switzerland), 9(2). https://doi.org/10.3390/su9020258

Press, W. H., Teukolsky, S. A., Vetterling, W. T., \& Flannery, B. P. (2007). Numerical recipes. The art of scientific computing. Journal of Experimental Psychology: General (3rd ed.). Cambridge, New York, Melbourne, Madrid, Cape Town, Singapore, São Paulo: Cambridge University Press.

Profloresta. (2008). Regional forest study in the forest management unit 1008. Durango, Mexico.

QGIS Development Team. (2019). QGIS Geographic Information System. Open Source Geospatial Foundation Project. Retrieved from http://www.qgis.org/

Qi, J., Chehbouni, A., Huete, A. R., Kerr, Y. H., \& Sorooshian, S. (1994). A Modified Soil Adjusted Vegetation Index. Remote Sensing of Environment, 48, 119-126.

Qin, L., Liu, Q., Zhang, M., \& Saeed, S. (2019). Effect of measurement errors on the estimation of tree biomass. Canadian Journal of Forest Research, 49, 1371-1378. https://doi.org/10.1139/cjfr-2019-0034

Quegan, S., Le Toan, T., Chave, J., Dall, J., Papathanassiou, K., Rocca, F., ... Williams, M. (2012). Report for Mission Selection: Biomass. Noordwijk, The Netherlands.

Quiñones-Pérez, C. Z., Silva-Flores, R., \& Wehenkel, C. (2012). Ecology of mexican fir Abies durangensis Martínez. Journal of Forestry Faculty, 12(3 (Special Iss.)), 180-184.

R Core Team. (2018). R: A language and environment for statistical computing. R Foundation for Statistical Computing, Vienna, Austria. https://doi.org/10.1016/j.jssas.2015.06.002

Rao, V. S., \& Rao, B. R. P. (2015). Carbon sequestration potential of tropical deciduous forests of Nallamalais, India. Asian J. Plant. Sci. Res., 5(3), 24-33.

Red Mex-SMIC. (2015). Protocol for forest carbon dynamics estimation in sites of intensive measurement : multi-scale approach. Mexico: Reinforcing REDD+ and South-South Cooperation.

Reed, D., \& Tomé, M. (1998). Total aboveground biomass and net dry matter accumulation by plant component in young Eucalyptus globulus in response to irrigation. Forest Ecology and Management, 103(1), 21-32. https://doi.org/10.1016/S0378-1127(97)00174-6

Rees, C. E. (1984). Error propagation calculations. Geochimica et Cosmochimica Acta, 48(11), $2309-2311$. https://doi.org/10.1016/0016-7037(84)90226-6

Riaño, D., Chuvieco, E., Salas, J., \& Aguado, I. (2003). Assessment of Different Topographic Corrections in Landsat-TM Data for Mapping Vegetation Types. IEEE Transactions on Geoscience and Remote Sensing, 41(5), 1056-1061.

Ricker, M., Calónico, J., Chávez, N., Gernandt, D. S., Gutiérrez, G., Martínez, E. M., ... Salazar, G. A. (2015). Taxonomic determination of herbarium specimens from the re-sampling of the national forest and soil inventory 2009-2013 (year 2013). Mexico City, Mexico.

Rocha, W. F. C., \& Nogueira, R. (2012). Monte Carlo simulation for the evaluation of measurement uncertainty of pharmaceutical certified reference materials. J. Braz. Chem. Soc., 23(3), 385-391.

Rodríguez-Laguna, R., Jiménez-Pérez, J., Meza-Rangel, J., Aguirre-Calderón, O., \& Razo-Zarate, R. (2008). Carbon contained in a tropical sub-deciduous forest in the Biosphere Reserve "El Cielo", Tamaulipas, Mexico. Revista Latinoamericana de Recursos Naturales, 4(2), 215-222.

Rodríguez-Veiga, P., Saatchi, S., Tansey, K., \& Balzter, H. (2016). Magnitude, spatial distribution and uncertainty of forest biomass stocks in Mexico. Remote Sensing of Environment, 183, 265-281. https://doi.org/10.1016/j.rse.2016.06.004

Rodríguez-Veiga, P., Wheeler, J., Louis, V., Tansey, K., \& Balzter, H. (2017). Quantifying Forest Biomass Carbon Stocks From Space. Current Forestry Reports, 1-18. https://doi.org/10.1007/s40725-017-00525

Rodríguez Laguna, R., Jiménez Pérez, J., Aguirre Calderón, O. A., \& Treviño Garza, E. J. (2006). Estimation of carbon stored in a cloud forest in Tamaulipas Mexico. Ciencia UANL, IX(April-June), 179-188.

Rojas-García, F., De Jong, B. H. J., Martínez-Zurimendí, P., \& Paz-Pellat, F. (2015). Database of 478 allometric equations to estimate biomass for Mexican trees and forests. Annals of Forest Science, 72(6), 
835-864.

Rojas-García, F., De Jong, B. H. J., \& Paz-Pellat, F. (2015). Areas of opportunity for the development of allometric models to estimate biomass in plant species with distribution in Mexico. In F. Paz-Pellat \& J. Wong-González (Eds.), Current state of knowledge of the carbon cycle and their interactions in Mexico: Synthesis 2014 (p. 642). Texcoco, Mexico: Programa Mexicano del Carbono en colaboración con el Centro de Investigación y Estudios Avanzados del Instituto Politécnico Nacional, Unidad Mérida y el Centro de Investigación y Asistencia en Tecnología y Diseño del Estado de Jalisco.

Rondeux, J., \& Pauwels, D. (1998). Le Forestor Vertex : une nouvelle génération de dendromètres. Revue Forestière Française, (1), 59-64. https://doi.org/10.4267/2042/5513

Roxburgh, S. H., Paul, K. I., Clifford, D., England, J. R., \& Raison, R. J. (2015). Guidelines for constructing allometric models for the prediction of woody biomass: How many individuals to harvest? Ecosphere, 6(3), 1-27. https://doi.org/10.1890/ES14-00251.1

Rueda Sánchez, A., Gallegos Rodríguez, A., González Eguiarte, D., Ruiz Corral, A., Benavides Solorio, J. de D., López Alcocer, E., \& Acosta Mireles, M. (2015). Aboveground biomass estimation in plantations of Cedrela odorata L. and Swietenia macrophylla King. Revista Mexicana de Ciencias Forestales, 5(25), 8-17.

Safari, A., \& Sohrabi, H. (2016). Ability of Landsat-8 OLI derived texture metrics in estimating aboveground carbon stocks of coppice oak forests. International Archives of the Photogrammetry, Remote Sensing and Spatial Information Sciences - ISPRS Archives, 41(June), 751-754. https://doi.org/10.5194/isprsarchives-XLI-B8-751-2016

Saket, M., Altrell, D., Vuorinen, P., Dalsgaard, S., \& Andersson, L. G. B. (2004). National Forest Inventory Field Manual Template.

Sarker, L. R., \& Nichol, J. E. (2011). Improved forest biomass estimates using ALOS AVNIR-2 texture indices. Remote Sensing of Environment, 115(4), 968-977. https://doi.org/10.1016/j.rse.2010.11.010

Schmid, W. A., \& Lazos Martínez, R. J. (2000). Guide to estimate the measurement uncertainty. National Center of Metrology, Mexico (CENAM). Queretaro, Qro., Mexico, Mexico.

Schreuder, H. T., \& Geissler, P. H. (1999). Plot Designs for Ecological Monitoring of Forest and Range. In C. Aguirre-Bravo \& C. Rodriguez Franco (Eds.), The North American Science Symposium:Toward a Unified. Framework for Inventorying and Monitoring Forest Ecosystem Resources (pp. 180-185). Guadalajara, Jalisco, Mexico: USDA Forest Service.

Scott, C. T. (1993). Optimal design of a plot cluster for monitoring. In K. Rennolls \& R. Gertner (Eds.), The optimal design of forest experiment and forest survey: Proceedings, IUFRO S.4.11 (pp. 233-242). London, UK: University of Greenwich.

Sediva, Sana, Uher, M., \& Havlikova, M. (2015). Application of the Monte Carlo Method to Estimate the Uncertainty of Air Flow Measurement. In I. Petráš, I. Podlubny, J. Kačur, \& J. Vásárhelyi (Eds.), 16th International Carpathian Control Conference (ICCC) (pp. 465-469). Szilvásvárad, Hungary: IEEE, ICCC.

Sediva, Sona, \& Havlikova, M. (2013). Comparison of GUM and Monte Carlo method for evaluation measurement uncertainty of indirect measurements. Proceedings of the 2013 14th International Carpathian Control Conference, ICCC 2013, (June), 325-329. https://doi.org/10.1109/CarpathianCC.2013.6560563

SEMARNAT. (2004). Strategies governing the National Forest and Soil Inventory. Guadalajara, Jalisco, Mexico.

SEMARNAT. (2014). Study of the Forest Supply Basin “Centro Occidente” of the State of Durango. Durango, Durango.

SEMARNAT, \& INECC. (2017). Methodological improvements for state inventories of GHG emissions and removals by land use, the evaluation of the utility and concordance for the national MRV system and its contribution in the commitments determined at national level. Zapopan, Jalisco, Mexico.

SEMARNAT, \& SHCP. (2009). The Economy of Climate Change in Mexico. Mexico City, Mexico.

Shi, L., \& Liu, S. (2017). Methods of estimating forest biomass: A review. In J. S. Tumuluru (Ed.), Biomass volume estimation and valorization for energy (1st ed., pp. 23-46). Intech open.

Sinha, S., Jeganathan, C., Sharma, L. K., \& Nathawat, M. S. (2015). A review of radar remote sensing for biomass estimation. International Journal of Environmental Science and Technology, 12(5), 17791792.

Solano, D., Vega, C., Eras, V. H., \& Cueva, K. (2014). Generation of models allometric to determine aerial biomass at the species level, by the destructive method of low intensity for the layer of forest dry Pluviestacional of Ecuador. Revista CEDAMAZ, 4(1), 32-44. 
Sousa, A. M. O., Gonçalves, A. C., \& Marques da Silva, J. R. (2017). Above-ground biomass estimation with high spatial resolution satellite images. In J. S. Tumuluru (Ed.), Biomass volume estimation and valorization for energy (1st ed., pp. 47-70). IntechOpen.

Spiess, A. N. (2015). Propagate: Propagation of Uncertainty. R package version 1.0-6.

Taylor, J. R. (1997). An Introduction to Error Analysis: The Study of Uncertainties in Physical Measurements. (A. McGuire, Ed.) (2nd ed., Vol. 9). Sausalito, California, US: University Science Books.

Teillet, P. M., Guindon, B., \& Goodenough, D. G. (1982). On the slope-aspect correction of multispectral scanner data. Canadian Journal of Remote Sensing, 8(2), 84-106. https://doi.org/10.1080/07038992.1982.10855028

Tiedeman, C. R., \& Green, C. T. (2013). Effect of correlated observation error on parameters, predictions, and uncertainty. Water Resources Research, 49(10), 6339-6355. https://doi.org/10.1002/wrcr.20499

Tomppo, E., Gschwantner, T., Lawrence, M., \& McRoberts, R. E. (2010). National Forest Inventories. Pathways for common reporting. (E. Tomppo, T. Gschwantner, M. Lawrence, \& R. E. McRoberts, Eds.) (First). Heidelberg Dordrecht London New York: Springer, Dordrecht. https://doi.org/10.1007/978-90-481-3233-1

United Nations. (2008). Non-sampling errors in haushold surveys. In United Nations (Ed.), Designing Household Survey Samples: Practical Guidelines Department of Economic and Social Affairs (1st ed., pp. 163-176). New York, US: United Nations Publication. Retrieved from http://www.ihsn.org/node/538

USGS. (2017). Product Guide: Landsat Surace Reflactance-Derived Spectral Indices. Montana, USA. https://doi.org/10.1016/0042-207X(74)93024-3

Van Breugel, M., Ransijn, J., Craven, D., Bongers, F., \& Hall, J. S. (2011). Estimating carbon stock in secondary forests: Decisions and uncertainties associated with allometric biomass models. Forest Ecology and Management, 262(8), 1648-1657. https://doi.org/10.1016/j.foreco.2011.07.018

Vargas-Larreta, B., López-Sánchez, C. A., Corral-Rivas, J. J., López-Martínez, J. O., Aguirre-Calderón, C. G., \& Álvarez-González, J. G. (2017). Allometric equations for estimating biomass and carbon stocks in the temperate forests of north-western Mexico. Forests, 8(269), 20.

Vázquez-Jiménez, R., Romero-Calcerrada, R., Ramos-Bernal, R., Arrogante-Funes, P., \& Novillo, C. (2017). Topographic Correction to Landsat Imagery through Slope Classification by Applying the SCS + C Method in Mountainous Forest Areas. ISPRS International Journal of Geo-Information, 6(9), 287. https://doi.org/10.3390/ijgi6090287

Velasco Bautista, E., Moreno Sánchez, F., \& Rodriguez P., R. (2002). Comparison of seven designs of secondary sampling units in forest inventories. Ciencia Forestal En México, 27(92), 29-51.

Wakeham, J. (2015). Uncertainty: History of the Concept. International Encyclopedia of the Social \& Behavioral Sciences: Second Edition (Second Edi). Elsevier. https://doi.org/10.1016/B978-0-08097086-8.03175-5

Walker, W. E., Harremoës, P., Rotmans, J., Van Der Sluijs, J. P., Van Asselt, M. B. A., Janssen, P., \& Von Krauss, M. P. K. (2003). Defining uncertainty. A Conceptual basis for uncertainty management in model-based decision support. Integrated Assessment, (4), 13. Retrieved from https://repository.tudelft.nl/islandora/object/uuid:fdc0105c-e601-402a-8f16ca97e9963592? collection=research

Wang, Y., Zhang, K., Tang, C., Cao, Q., Tian, Y., Zhu, Y., ... Liu, X. (2019). Estimation of rice growth parameters based on linear mixed-effect model using multispectral images from fixed-wing unmanned aerial vehicles. Remote Sensing, 11(11). https://doi.org/10.3390/rs11111371

Weaver, S. A., Ucar, Z., Bettinger, P., Merry, K., Faw, K., \& Cieszewski, C. J. (2015). Assessing the Accuracy of Tree Diameter Measurements Collected at a Distance. Croatian Journal of Forest Engineering, 36(1), 73-84.

West, P. W. (2009). Tree and Forest Measurement. Springer (Vol. 53). https://doi.org/10.1017/CBO9781107415324.004

Wiemann, M. C., \& Williamson, G. B. (2013). Biomass determination using wood specific gravity from increment cores. General Technical Report FPL-GTR-225. Wisconsin, US.

Williams, M. S., Bechtold, W. A., \& LaBau, V. J. (1994). Five Instruments for Measuring Tree Height: An Evaluation. Southern Journal of Applied Forestry, 18(2), 76-82. https://doi.org/10.1093/sjaf/18.2.76

Wilson, E. H., \& Sader, S. A. (2002). Detection of forest harvest type using multiple dates of Landsat TM imagery. Remote Sensing of Environment, 80(3), 385-396. https://doi.org/10.1016/S00344257(01)00318-2 
Wing, M. G., Kellog, L., \& Solmie, D. (2004). Comparing digital range finders for forestry applications. Journal of Forestry, 102(4), 16-20. https://doi.org/10.1093/jof/102.4.16

Wu, C., Shen, H., Wang, K., Shen, A., Deng, J., \& Gan, M. (2016). Landsat imagery-based above ground biomass estimation and change investigation related to human activities. Sustainability (Switzerland), 8(2). https://doi.org/10.3390/su8020159

Yanai, R. D., Battles, J. J., Richardson, A. D., Blodgett, C. a., Wood, D. M., \& Rastetter, E. B. (2010). Estimating uncertainty in ecosystem budget calculations. Ecosystems, 13(2), 239-248. https://doi.org/10.1007/s10021-010-9315-8

Young, N. E., Anderson, R. S., Chignell, S. M., Vorster, A. G., Lawrence, R., \& Evangelista, P. H. (2017). A survival guide to Landsat preprocessing. Ecology, 98(4), 920-932.

Zhang, G., Ganguly, S., Nemani, R. R., White, M. A., Milesi, C., Hashimoto, H., ... Myneni, R. B. (2014). Estimation of forest aboveground biomass in california using canopy height and leaf area index estimated from satellite data. Remote Sensing of Environment, 151, 44-56. https://doi.org/10.1016/j.rse.2014.01.025

Zhao, P., Lu, D., Wang, G., Wu, C., Huang, Y., \& Yu, S. (2016). Examining spectral reflectance saturation in Landsat imagery and corresponding solutions to improve forest aboveground biomass estimation. Remote Sensing, 8(6), 26. https://doi.org/10.3390/rs8060469

Zheng, D., Heath, L. S., \& Ducey, M. J. (2008). Spatial distribution of forest aboveground biomass estimated from remote sensing and forest inventory data in New England, USA. Journal of Applied Remote Sensing, 2(1), 17. https://doi.org/10.1117/1.2940686

Zheng, D., Rademacher, J., Chen, J., Crow, T., Bresee, M., Le Moine, J., \& Ryu, S. R. (2004). Estimating aboveground biomass using Landsat $7 \mathrm{ETM}+$ data across a managed landscape in northern Wisconsin, USA. Remote Sensing of Environment, 93(3), 402-411. https://doi.org/10.1016/j.rse.2004.08.008 


\section{Chapter VIII: Appendix}

\section{VIII.1 Appendix I}

\begin{tabular}{|c|c|c|c|c|c|c|c|c|c|c|c|c|c|c|c|c|c|c|c|c|c|c|}
\hline $\begin{array}{c}\text { Vegetation } \\
\text { Group / Genus / } \\
\text { Specie }\end{array}$ & $\begin{array}{c}\text { Tree } \\
\text { No. } \\
\text { MNFI } \\
04-09\end{array}$ & $\begin{array}{rr}\text { Tree } & \\
\text { No. } & \text { State } \\
\text { MNFI } & \\
09-14 & \\
\end{array}$ & $\mathrm{n}$ & $R^{2}$ & $\begin{array}{c}R M S E \\
(\mathrm{~kg})\end{array}$ & $\begin{array}{l}D B H \\
\text { range } \\
(\mathrm{cm})\end{array}$ & $\begin{array}{c}T H \\
\text { range } \\
(\mathrm{m})\end{array}$ & $\begin{array}{c}W \\
(\mathrm{~kg})\end{array}$ & $\alpha$ & $\begin{array}{c}L n \\
\alpha\end{array}$ & $\begin{array}{c}\beta I \\
L n \\
D B H \\
\end{array}$ & $\begin{array}{l}\beta 2 \\
L n \\
T H \\
\end{array}$ & $\begin{array}{c}\beta 3 \\
L n \\
G^{*} T H \\
\end{array}$ & $\begin{array}{l}\beta 4 \\
L n \\
G \\
\end{array}$ & $\begin{array}{c}\beta 5 \\
L n \\
D B H^{2} T H \\
\end{array}$ & $\begin{array}{c}\beta 6 \\
\mathrm{Ln} \\
\mathrm{CVol} \\
\end{array}$ & $\begin{array}{c}\beta 7 \\
D B H\end{array}$ & $\begin{array}{l}\beta 8 \\
T H\end{array}$ & $\begin{array}{c}\beta 9 \\
D B H^{2} T H\end{array}$ & $\begin{array}{l}\beta 10 \\
\text { log } \\
\text { TH } \\
\end{array}$ & $\begin{array}{cc}\beta 11 & \beta 12 \\
\log & \beta B H^{2} \\
D B H & \\
\end{array}$ & Source \\
\hline Abies $s p$ & 108 & $\begin{array}{l}40 \text { Tlaxcala, } \\
\text { MX }\end{array}$ & 26 & 0.99 & ND & $5.7-79$ & $6.8-45 \quad W_{t}=\alpha D B H^{\beta l}$ & Total & & $\exp ^{\wedge}(0.071)$ & 2.510 & & & & & & & & & & & $\begin{array}{l}\text { Avendaño } \\
\text { Hernandez et } \\
\text { al., 2009 }\end{array}$ \\
\hline Alnus spp & 1011 & 952 Oaxaca, & 52 & 0.88 & $\mathrm{ND}$ & $\begin{array}{r}3.9- \\
23.5 \\
\end{array}$ & $\mathrm{ND}^{W_{t}=\alpha D B H^{\beta l}}$ & Total & & $\exp ^{\wedge}(-2.14)$ & 2.23 & & & & & & & & & & & $\begin{array}{l}\text { Acosta } \\
\text { Mireles et al, }\end{array}$ \\
\hline Inga spp & 0 & $5^{\mathrm{Mx}}$ & 52 & 0.97 & ND & $3.3-25$ & ND $W_{t}=\alpha D B H^{\beta 1}$ & Total & & $\exp ^{\wedge}(-1.76)$ & 2.26 & & & & & & & & & & & 2002 \\
\hline Clethra sp & 0 & 5 MX & 15 & 0.95 & ND & $\begin{array}{l}5.3- \\
23.3\end{array}$ & $\mathrm{ND}{ }^{W_{t}=\alpha D B H^{\beta 1}}$ & Total & & $\exp ^{\wedge}(0.463)$ & 1.1817 & & & & & & & & & & & $\begin{array}{l}\text { Acosta- } \\
\text { Mireles et al., } \\
2011 \\
\end{array}$ \\
\hline Carya spp & 21 & 3 & 20 & 0.98 & ND & ND & ND $W_{t}=\alpha D B H^{\beta I}$ & Total & & $\exp ^{\wedge}(0.061)$ & 2.532 & & & & & & & & & & & Rodríguez \\
\hline Ficus sp & 0 & $\frac{4}{4}$ & 143 & 0.92 & ND & ND & ND $W_{t}=\alpha D B H^{\beta 1}$ & Total & & $\exp ^{\wedge}(0.027)$ & 2.864 & & & & & & & & & & & Laguna et al., \\
\hline Nectandra $s p$ & 3 & {$[\overline{0}$ Tamps, MX } & 20 & 0.95 & ND & $\mathrm{ND}$ & ND $W_{t}=\alpha D B H^{\beta l}$ & Total & & $\exp ^{\wedge}(0.004)$ & 3.357 & & & & & & & & & & & \\
\hline Piscidia $s p$ & 2 & 0 & 30 & 0.95 & ND & $\mathrm{ND}$ & ND $W_{t}=\alpha D B H^{\beta I}$ & Total & & $\exp ^{\wedge}(0.064)$ & 2.623 & & & & & & & & & & & Rodríguez- \\
\hline Psidium spp & 3 & 4 & 18 & 0.99 & ND & ND & ND $W_{t}=\alpha D B H^{\beta 1}$ & Total & & $\exp ^{\wedge}(0.247)$ & 2.25 & & & & & & & & & & & Laguna et al., \\
\hline Tilia spp & 346 & $\overline{462}$ & 10 & 0.99 & $\mathrm{ND}$ & $\mathrm{ND}$ & ND $W_{t}=\alpha D B H^{\beta l}$ & Total & & $\exp ^{\wedge}(0.048)$ & 2.582 & & & & & & & & & & & 2008 \\
\hline Cupressus spp & 361 & $\begin{array}{l}\text { Mexico } \\
\text { City, MX } \\
\end{array}$ & 18 & 0.93 & ND & $3-45$ & $\mathrm{ND}{ }^{W_{t}=\alpha D B H^{\beta I}}$ & Total & & $\exp ^{\wedge}(0.527)$ & 1.7712 & & & & & & & & & & & Rojas-García \\
\hline Prunus spp & 86 & $\begin{array}{l}109 \begin{array}{l}\text { Oaxaca, } \\
\text { MX }\end{array} \\
\end{array}$ & 11 & 0.95 & ND & ND & $\mathrm{ND}{ }^{W_{t}=\alpha D B H^{\beta I}}$ & Total & & $\exp ^{\wedge}(-2.76)$ & 2.37 & & & & & & & & & & & et al., 2015a \\
\hline Ostrya $s p$ & 0 & 2 US & 55 & 0.81 & 54.45 & $3-47$ & ND $W_{t}=\alpha D B H^{\beta 1}$ & Total & & $\exp ^{\wedge}(0.104)$ & 2.535 & & & & & & & & & & & Chojnacky et \\
\hline Picea $s p$ & 0 & 29 US & 289 & 0.81 & 107.19 & $3-72$ & ND $W_{t}=\alpha D B H^{\beta I}$ & Total & & $\exp ^{\wedge}(0.118)$ & 2.323 & & & & & & & & & & & al., 2014 \\
\hline Prosopis spp & 34 & $\begin{array}{l}\text { Dgo., Chih., } \\
83 \text { Coah., Zac., } \\
\text { MX }\end{array}$ & 30 & 0.98 & ND & $\begin{array}{r}5.2- \\
31.5\end{array}$ & $\begin{array}{l}2.6-W_{l}=\alpha D B H^{\beta I} \\
6.4\end{array}$ & Total & & $\exp ^{\wedge}(0.056)$ & 2.383 & & & & & & & & & & & $\begin{array}{l}\text { Méndez } \\
\text { González et } \\
\text { al. , 2012 } \\
\end{array}$ \\
\hline Pseudotsuga spp & 543 & $\begin{array}{l}560 \text { Chih.,Dgo., } \\
\text { MX }\end{array}$ & 81 & 0.94 & 83.71 & $\begin{array}{l}8.4- \\
49.8 \\
\end{array}$ & $\mathrm{ND}^{W_{t}=\alpha D B H^{\beta l}}$ & Total & & $\exp ^{\wedge}(0.135)$ & 2.303 & & & & & & & & & & & José Návar, \\
\hline $\begin{array}{l}\text { Tropical dry } \\
\text { forest }\end{array}$ & 562 & $\begin{array}{l}728 \text { Sinaloa, } \\
\text { MX }\end{array}$ & 39 & 0.85 & 52.41 & $\begin{array}{r}5.2- \\
32.6 \\
\end{array}$ & $\mathrm{ND}^{W_{l}=\alpha D B H^{\beta l}}$ & Total & & $\exp ^{\wedge}(0.37)$ & 1.96 & & & & & & & & & & & 2009 \\
\hline Tabebuia sp & 0 & $10 \mathrm{EC}$ & 94 & 0.91 & 1.03 & $\mathrm{ND}$ & ND $W_{i}=\alpha D B H^{\beta l} T H^{\beta 2}$ & Total & & $\exp ^{\wedge}(0.057)$ & 1.98 & 1.06 & & & & & & & & & & $\begin{array}{l}\text { Solano et al., } \\
2014\end{array}$ \\
\hline Abarema sp & 0 & 12 Pará, BR & 82 & 0.92 & 1.11 & $5-8.8$ & 6-8.2 $W_{t}=\alpha\left(G^{*} T H\right)^{\beta 3}$ & Total & & $\exp ^{\wedge}(387.8)$ & & & 0.892 & & & & & & & & & $\begin{array}{l}\text { Ducey et al., } \\
2009\end{array}$ \\
\hline
\end{tabular}

Table VIII-1. AGB allometric models $(A M)$ used in $M N F I$ in the state of Durango with the number of trees registered. $A M$ data: $\mathrm{n}=$ number of sampled trees to fit the $A M ; R^{2}=$ coefficient of determination; $R M S E=$ root-mean-square error; $W=\mathrm{AGB}$ in $\mathrm{kg}$ per tree; Variables used to fit the $A M$ : $\mathrm{DBH}, \mathrm{TH}$, basal area $(G)$, crown volume $(\mathrm{CVol})$; $\mathrm{ND}$ (= no data) is given when data were not reported; $\mathrm{BR}=\mathrm{Brazil}, \mathrm{EC}=\mathrm{Ecuador}$, $\mathrm{MX}=\mathrm{Mexico}$, US $=$ United States. 
Chapter VIII: Appendix

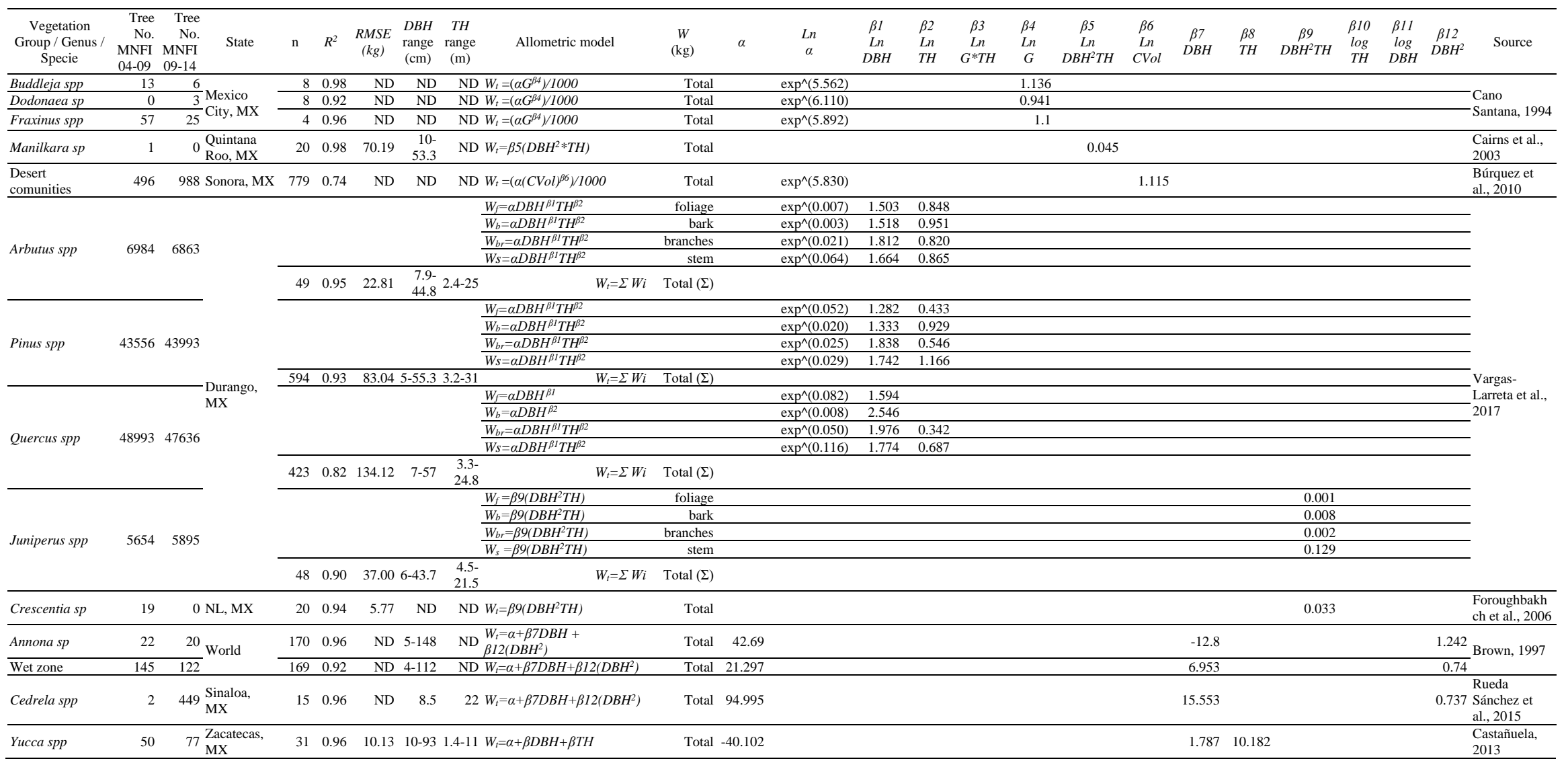

Table VIII-1. Continuation ..... 


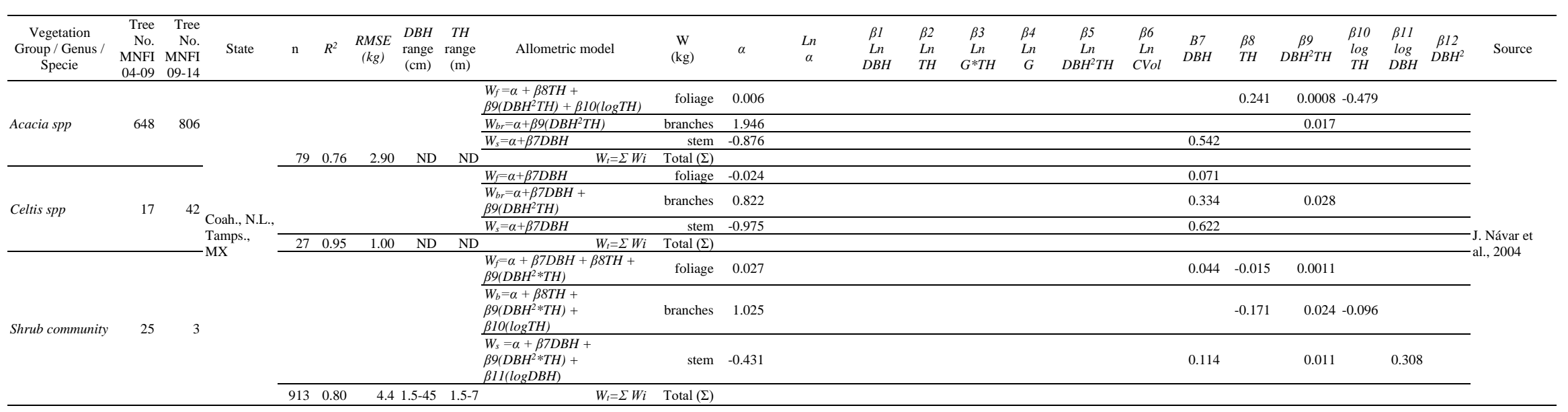

Table VIII-1. Continuation ..... 


\section{VIII.2 Appendix II}

\begin{tabular}{|c|c|c|c|}
\hline $\begin{array}{l}D B H \\
\text { class }\end{array}$ & $\begin{array}{c}\text { Normal } \\
\text { distribution } \\
\text { parameters } \\
(\widehat{\mu}, \widehat{\sigma})\end{array}$ & $\begin{array}{c}\text { Best-fitted distribution } \\
\text { parameters }\end{array}$ & Goodness-of-fit \\
\hline$[7.5-56]$ & $(0,0.368)$ & $\begin{array}{l}\text { Johnson SU: } \gamma=0, \delta=1.073 \text {, } \\
\xi=0, \lambda=0.371\end{array}$ & $\begin{array}{l}B I C=-575.099, R S S=4.38 \mathrm{e}^{-06}, \\
M S E=8.83 \mathrm{e}^{-05}\end{array}$ \\
\hline$[7.5-10)$ & $(0,0.240)$ & $\begin{array}{l}\text { Scaled } / \text { shifted t: } \hat{\mu}=0, \\
\mathrm{sd}=0.218, \mathrm{df}=2.472\end{array}$ & $\begin{array}{l}B I C=-97.756, R S S=2.871 \mathrm{e}^{-04}, \\
M S E=0.008\end{array}$ \\
\hline$[10-15)$ & $(0,0.330)$ & $\begin{array}{l}\text { Johnson SU: } \gamma=0, \delta=1.108, \\
\xi=0, \lambda=0.344\end{array}$ & $\begin{array}{l}B I C=-497.681, R S S=5.030 \mathrm{e}^{-05}, \\
M S E=0.0002\end{array}$ \\
\hline$[15-20)$ & $(0,0.373)$ & $\begin{array}{l}\text { Johnson SU: } \gamma=0, \delta=1.228 \text {, } \\
\xi=0, \lambda=0.436\end{array}$ & $\begin{array}{l}B I C=-449.72, R S S=4.215 \mathrm{e}^{-05}, \\
M S E=0.0013\end{array}$ \\
\hline$[20-25)$ & $(0,0.442)$ & $\begin{array}{l}\text { Johnson SU: } \gamma=0, \delta=1.327, \\
\xi=0, \lambda=0.567\end{array}$ & $\begin{array}{l}B I C=-393.741, R S S=6.009 \mathrm{e}^{-05}, \\
M S E=0.0006\end{array}$ \\
\hline$[25-30)$ & $(0,0.540)$ & $\begin{array}{l}\text { Johnson SU: } \gamma=0, \delta=-1.907, \\
\xi=0, \lambda=-1.013\end{array}$ & $\begin{array}{l}B I C=-290.398, R S S=3.470 \mathrm{e}^{-04}, \\
M S E=0.0011\end{array}$ \\
\hline$[30-35)$ & $(0,0.748)$ & Laplace: $\hat{\mu}=0, \hat{\sigma}=1.040$ & $\begin{array}{l}B I C=-116.481, R S S=2.149 \mathrm{e}^{-02}, \\
M S E=0.018\end{array}$ \\
\hline$[35-56]$ & $(0,1.197)$ & Laplace: $\hat{\mu}=0, \hat{\sigma}=1.197$ & $\begin{array}{l}B I C=-148.022, R S S=0.018, \\
M S E=0.0177\end{array}$ \\
\hline
\end{tabular}

Table VIII-2. Parameters and goodness-of-fit for the PDF fitted (normal distribution and best-fitted distribution) for $D B H$ measurement by $D B H$ class in El Salto, Durango, Mexico.

\begin{tabular}{|c|c|c|c|}
\hline $\begin{array}{c}T H \\
\text { class }\end{array}$ & $\begin{array}{c}\text { Normal } \\
\text { distribution } \\
\text { parameters } \\
(\widehat{\mu}, \widehat{\sigma})\end{array}$ & $\begin{array}{l}\text { Best-fitted distribution } \\
\text { parameters }\end{array}$ & Goodness-of-fit \\
\hline [3.6-28] & $(0,0.573)$ & $\begin{array}{l}\text { Johnson SU: } \gamma=0, \delta=0.830 \text {, } \\
\xi=0, \lambda=0.437\end{array}$ & $\begin{array}{l}B I C=-442.844, R S S=1.156 \mathrm{e}^{-05}, \\
M S E=0.00018\end{array}$ \\
\hline$[3.6-10)$ & $(0,0.351)$ & $\begin{array}{l}\text { Johnson SU: } \gamma=0, \delta=0.987, \\
\xi=0, \lambda=0.325\end{array}$ & $\begin{array}{l}B I C=-367.425, R S S=3.984 \mathrm{e}^{-05}, \\
M S E=0.0004\end{array}$ \\
\hline$[10-12.5)$ & $(0,0.472)$ & $\begin{array}{l}\text { Johnson SU: } \gamma=0, \delta=0.942, \\
\xi=0, \lambda=0.425\end{array}$ & $\begin{array}{l}B I C=-330.029, R S S=1.357 \mathrm{e}^{-04}, \\
M S E=0.00064\end{array}$ \\
\hline$[12.5-15)$ & $(0,0.605)$ & $\begin{array}{l}\text { Johnson SU: } \gamma=0, \delta=1.094, \\
\xi=0, \lambda=0.629\end{array}$ & $\begin{array}{l}B I C=-311.550, R S S=3.133 \mathrm{e}^{-05}, \\
M S E=0.0009\end{array}$ \\
\hline$[15-17.5)$ & $(0,0.796)$ & $\begin{array}{l}\text { Johnson SU: } \gamma=0, \delta=0.938, \\
\xi=0, \lambda=0.682\end{array}$ & $\begin{array}{l}B I C=-277.538, R S S=2.166 \mathrm{e}^{-06}, \\
M S E=0.0013\end{array}$ \\
\hline$[17.5-20)$ & $(0,0.982)$ & $\begin{array}{l}\text { Scaled/shifted t: } \hat{\mu}=0, \\
\hat{\sigma}=0.982, \mathrm{df}=2.472\end{array}$ & $\begin{array}{l}B I C=-218.041, R S S=0.0014, \\
M S E=0.0024\end{array}$ \\
\hline [20-28] & $(0,1.040)$ & Laplace: $\hat{\mu}=0, \hat{\sigma}=2.095$ & $\begin{array}{l}B I C=-39.495, R S S=0.0025, \\
M S E=0.040\end{array}$ \\
\hline
\end{tabular}

Table VIII-3. Parameters and goodness-of-fit for the PDF fitted (normal distribution and best-fitted distribution) for $T H$ measurement by $T H$ class in El Salto, Durango, Mexico. 
VIII.3 Appendix III

\begin{tabular}{|c|c|c|c|}
\hline $\begin{array}{l}D B H \\
\text { class }\end{array}$ & $\begin{array}{c}\text { Normal } \\
\text { distribution } \\
\text { parameters } \\
(\widehat{\mu}, \widehat{\sigma})\end{array}$ & $\begin{array}{c}\text { Best-fitted distribution } \\
\text { parameters }\end{array}$ & Goodness-of-fit \\
\hline [7-64] & $(0,0.212)$ & Logistic: $\hat{\mu}=0, \mathrm{~s}=0.132$ & $\begin{array}{l}B I C=70.854, R S S=0.009, \\
M S E=0.1024\end{array}$ \\
\hline$[7-20)$ & $(0,0.150)$ & Normal: $\hat{\mu}=0, \hat{\sigma}=0.149$ & $\begin{array}{l}B I C=433.360, \quad R S S=0.131, \\
M S E=1.216\end{array}$ \\
\hline$[20-40)$ & $(0,0.283)$ & $\begin{array}{l}\text { Curvilinear Trapezoidal: } \\
\mathrm{a}=-0.441, \mathrm{~b}=0.441, \mathrm{~d}=0.242\end{array}$ & $\begin{array}{l}B I C=175.396, R S S=0.179 \\
M S E=0.273\end{array}$ \\
\hline$[40-64)$ & $(0,0.436)$ & Cosine: $\mathrm{mu}=0, \hat{\sigma}=1.102$ & $\begin{array}{l}B I C=137.652, R S S=1.5497, \\
M S E=0.278\end{array}$ \\
\hline
\end{tabular}

Table VIII-4. Parameters and goodness-of-fit for the PDF fitted (normal distribution and best-fitted distribution) for $D B H$ measurement made with Caliper by $D B H$ class in Göttingen, Germany.

\begin{tabular}{|c|c|c|c|}
\hline $\begin{array}{l}D B H \\
\text { class }\end{array}$ & $\begin{array}{c}\text { Normal } \\
\text { distribution } \\
\text { parameters } \\
(\widehat{\mu}, \widehat{\sigma})\end{array}$ & $\begin{array}{c}\text { Best-fitted Dn } \\
\text { parameters }\end{array}$ & Goodness-of-fit \\
\hline [7-64] & $(0,0.209)$ & $\begin{array}{l}\text { Logistic: } \text { Location }=0, \\
\text { scale }=0.128\end{array}$ & $\begin{array}{l}B I C=365.781, R S S=0.021, \\
M S E=0.910\end{array}$ \\
\hline$[7-20)$ & $(0,0.133)$ & $\begin{array}{l}\text { Logistic: location }=0, \\
\text { scale }=0.084\end{array}$ & $\begin{array}{l}B I C=415.526, R S S=0.520, \\
M S E=1.568\end{array}$ \\
\hline$[20-40)$ & $(0,0.266)$ & $\begin{array}{l}\text { Normal: } \hat{\mu}=0 \text {, } \\
\hat{\sigma}=0.266\end{array}$ & $\begin{array}{l}B I C=367.917, R S S=0.581, \\
M S E=0.967\end{array}$ \\
\hline$[40-64)$ & $(0,0.350)$ & $\begin{array}{l}\text { Curvilinear Trapezoidal: } \\
\mathrm{a}=-0.506, \mathrm{~b}=0.506, \mathrm{~d}=0.251\end{array}$ & $\begin{array}{l}B I C=370.031, R S S=0.466, \\
M S E=1.038\end{array}$ \\
\hline
\end{tabular}

Table VIII-5. Parameters and goodness-of-fit for the PDF fitted (normal distribution and best-fitted distribution) for $D B H$ measurement made with Tape by $D B H$ class in Göttingen, Germany.

\begin{tabular}{ccll}
\hline $\begin{array}{c}\boldsymbol{T H} \\
\text { class }\end{array}$ & $\begin{array}{c}\text { Normal } \\
\text { distribution } \\
\text { parameters }\end{array}$ & \multicolumn{1}{c}{$\begin{array}{c}\text { Best-fitted distribution } \\
\text { parameters }\end{array}$} & \multicolumn{1}{c}{ Goodness-of-fit } \\
& $(\widehat{\boldsymbol{\mu}}, \widehat{\boldsymbol{\sigma}})$ & & \\
\hline$[5-37]$ & $(0,1.229)$ & Trapezoidal: $\mathrm{a}=-2.162$, & $B I C=-128.960, R S S=0.0016$, \\
& & $\mathrm{b}=-1.062, \mathrm{c}=1.062, \mathrm{~d}=2.162$ & $M S E=0.011$ \\
{$[5-15)$} & $(0,0.933)$ & Cosine: $\mathrm{mu}=0, \hat{\sigma}=2.318$ & $B I C=-36.263, R S S=3.033 \mathrm{e}^{-03}$, \\
& & & $M S E=0.0301$ \\
{$[15-25)$} & $(0,1.427)$ & Curvilinear Trapezoidal: & $B I C=-43.896, R S S=0.028$, \\
& & $\mathrm{a}=-1.971, \mathrm{~b}=1.971, \mathrm{~d}=0.246$ & $M S E=0.029$ \\
{$[25-37)$} & $(0,1.505)$ & Uniform: $\min =-2.426$, & $B I C=-13.726, R S S=1.156 \mathrm{e}^{-02}$, \\
& & max $=2.426$ & $M S E=0.0418$ \\
\hline
\end{tabular}

Table VIII-6. Parameters and goodness-of-fit for the PDF fitted (normal distribution and best-fitted distribution) for $T H$ measurement made with clinometer by $T H$ class in Göttingen, Germany. 


\begin{tabular}{|c|c|c|c|}
\hline $\begin{array}{c}\mathrm{TH} \\
\text { class }\end{array}$ & $\begin{array}{c}\text { Normal } \\
\text { distribution } \\
\text { parameters } \\
(\widehat{\mu}, \widehat{\sigma})\end{array}$ & $\begin{array}{c}\text { Best-fitted distribution } \\
\text { parameters }\end{array}$ & Goodness-of-fit \\
\hline$[5-37]$ & $(0,0.646)$ & Cosine: $m u=0, \hat{\sigma}=1.641$ & $\begin{array}{l}B I C=-31.495, R S S=1.209 \mathrm{e}^{-04}, \\
M S E=0.033\end{array}$ \\
\hline$[5-15)$ & $(0,0.348)$ & Von Mises: $\mathrm{mu}=0, \mathrm{kappa}=8.519$ & $\begin{array}{l}B I C=207.933, R S S=0.063 \\
M S E=0.279\end{array}$ \\
\hline$[15-25)$ & $(0,0.835)$ & $\begin{array}{l}\text { Curvilinear Trapezoidal: } \\
\mathrm{a}=-1.048, \mathrm{~b}=1.048, \mathrm{~d}=0.236\end{array}$ & $B I C=3.176, R S S=0.024, M S E=0.0479$ \\
\hline$[25-37)$ & $(0,0.882)$ & Cosine: $\mathrm{mu}=0, \hat{\sigma}=2.180$ & $\begin{array}{l}B I C=4.276, R S S=8.643 \mathrm{e}^{-03}, \\
M S E=0.052\end{array}$ \\
\hline
\end{tabular}

Table VIII-7. Parameters and goodness-of-fit for the PDF fitted (normal distribution and best-fitted distribution) for $T H$ measurement made with Vertex by $T H$ class in Göttingen, Germany.
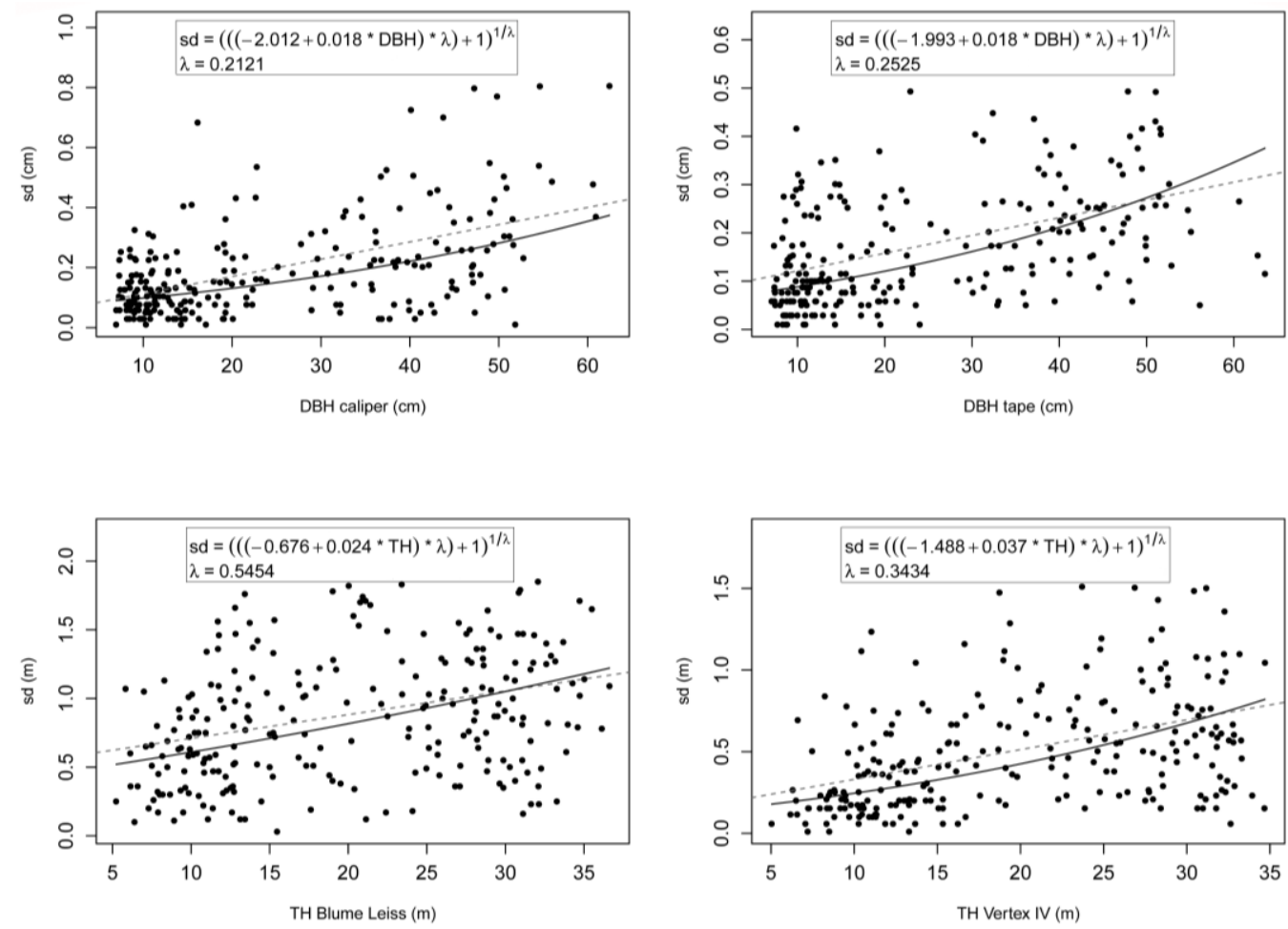

Figure VIII-1. Model fitted to estimate the measurement uncertainty, according to the tree size for Caliper (DBH), Tape (DBH), Blume-Leiss (TH) and Vertex IV (TH), with information of 250 trees in the study area of Göttingen. 
VIII.4 Appendix IV

\begin{tabular}{|c|c|c|c|c|c|c|c|c|c|c|c|}
\hline Scenario & Plot & 1 & 2 & 3 & 4 & 5 & 6 & 7 & 8 & 9 & 10 \\
\hline$N D n$ & $\mathrm{Mg} \mathrm{ha}^{-1}$ & 146.0 & 164.9 & 139.0 & 210.4 & 228.4 & 215.1 & 181.3 & 210.9 & 86.6 & 178.1 \\
\hline$N D n$ & $\mathrm{uMg} \mathrm{ha}{ }^{-1}$ & 3.74 & 5.05 & 6.10 & 6.40 & 3.00 & 4.10 & 3.93 & 5.26 & 5.26 & 4.73 \\
\hline$N D n C$ & $\mathrm{Mg} \mathrm{ha}^{-1}$ & 138.4 & 154.8 & 126.8 & 197.6 & 221.8 & 206.6 & 173.2 & 200.3 & 76.1 & 168.6 \\
\hline$N D n C$ & $\mathrm{uMg} \mathrm{ha}^{-1}$ & 3.79 & 5.07 & 6.10 & 6.41 & 3.31 & 4.26 & 4.04 & 5.29 & 5.26 & 4.77 \\
\hline$R M S D$ & $\mathrm{Mg} \mathrm{ha}^{-1}$ & 146.0 & 164.9 & 139.0 & 210.4 & 228.4 & 215.1 & 181.3 & 210.9 & 86.6 & 178.1 \\
\hline$R M S D$ & $\mathrm{uMg} \mathrm{ha} a^{-1}$ & 3.79 & 5.09 & 6.12 & 6.43 & 3.13 & 4.17 & 3.99 & 5.30 & 5.27 & 4.77 \\
\hline
\end{tabular}

Table VIII-8. AGB and uncertainty of the AGB calculation by plot using three scenarios for the measurement uncertainty estimation (NDn, $N D n C, R M S D)$ in El Salto, Durango, Mexico.

\begin{tabular}{|c|c|c|c|c|c|c|c|}
\hline & Df & Sum Sq & Error SS & den Df & F value & $\operatorname{Pr}(>\mathbf{F})$ & \\
\hline Intercept & 1 & 690.53 & 29.0125 & 9 & 214.209 & $1.396 \mathrm{e}-07$ & $* * *$ \\
\hline Scenario & 2 & 0.03 & 0.0449 & 18 & 5.548 & 0.01327 & $*$ \\
\hline \multicolumn{8}{|c|}{ Mauchly Tests for Sphericity } \\
\hline Scenario & $\begin{array}{r}\text { Test statistic } \\
0.028044 \\
\end{array}$ & $\begin{array}{l}\mathrm{p} \text {-value } \\
6.1853 \mathrm{e}-07\end{array}$ & & & & & \\
\hline \multicolumn{8}{|c|}{ Corrections for Departure from Sphericity } \\
\hline & GG eps & $\operatorname{Pr}(>\mathrm{F}[\mathrm{GG}])$ & & & \multirow{2}{*}{\multicolumn{3}{|c|}{ Greenhouse-Geisser }} \\
\hline Scenario & $\begin{array}{l}0.50711 \\
\text { H F eps }\end{array}$ & $\begin{array}{r}0.0422 \\
\operatorname{Pr}(>\mathrm{F}[\mathrm{HF}])\end{array}$ & $*$ & & & & \\
\hline Scenario & 0.5097947 & 0.04193635 & $*$ & & Huynh-F & & \\
\hline
\end{tabular}

Table VIII-9. Repeated-measures ANOVA, with correction for sphericity, applied to three scenarios (NDn, NDnC, RMSD) of measurement uncertainty estimation in AGB calculation in El Salto, Durango, Mexico.

\begin{tabular}{l|ccc}
\hline Scenario & Group (Holm correction) & NDn & NDnC \\
\hline$N D n$ & $\mathrm{~A}$ & - & - \\
$N D n C$ & $\mathrm{AB}$ & 0.0850 & - \\
$R M S D$ & $\mathrm{~B}$ & 0.0037 & 0.2766 \\
\hline
\end{tabular}

Table VIII-10. Pairwise comparison using paired T- Test, with p-value Holm's correction, for three scenarios (NDn, NDnC, RMSD) of AGB uncertainty estimation in El Salto, Durango, Mexico. 


\begin{tabular}{ll|llllllllll}
\hline Scenario & Plot & $\mathbf{1}$ & $\mathbf{2}$ & $\mathbf{3}$ & $\mathbf{4}$ & $\mathbf{5}$ & $\mathbf{6}$ & $\mathbf{7}$ & $\mathbf{8}$ & $\mathbf{9}$ & $\mathbf{1 0}$ \\
\hline$N D n C$ & $\mathrm{uMg} \mathrm{ha}^{-1}$ & 3.79 & 5.07 & 6.10 & 6.41 & 3.31 & 4.26 & 4.04 & 5.29 & 5.26 & 4.77 \\
$N D n C a$ & $\mathrm{uMg} \mathrm{ha}^{-1}$ & 0.41 & 0.39 & 0.29 & 0.40 & 0.88 & 0.72 & 0.55 & 0.46 & 0.20 & 0.44 \\
$N D n C b$ & $\mathrm{uMg} \mathrm{ha}^{-1}$ & 0.54 & 0.42 & 0.28 & 0.47 & 1.04 & 0.90 & 0.77 & 0.59 & 0.17 & 0.54 \\
$N D n C a b$ & $\mathrm{uMg} \mathrm{ha}^{-1}$ & 0.41 & 0.35 & 0.25 & 0.37 & 0.84 & 0.70 & 0.57 & 0.45 & 0.16 & 0.43 \\
$N D n C c$ & $\mathrm{uMg} \mathrm{ha}^{-1}$ & 3.70 & 5.03 & 6.08 & 6.37 & 2.90 & 4.04 & 3.88 & 5.22 & 5.25 & 4.70 \\
\hline \multicolumn{2}{l}{$N D n C=$ all sources, $N D n C a=D B H, N D n C b=T H, N D n C a b=$ correlation, $N D n C c=$ allometric model }
\end{tabular}

Table VIII-11. Uncertainty of the AGB calculation by plot using the estimation with all sources of measurement error $(\mathrm{NDnC})$ and by source ( $N D n C a, N D n C b, N D n C a b, N D n C c)$ in El Salto, Durango, Mexico.

\begin{tabular}{|c|c|c|c|c|c|c|c|}
\hline & Df & Sum Sq & Error SS & den Df & F value & $\operatorname{Pr}(>F)$ & \\
\hline Intercept & 1 & 8.294 & 1.0135 & 9 & 73.655 & $1.257 \mathrm{e}-05$ & **** \\
\hline Scenario & 4 & 87.528 & 5.1194 & 36 & 153.877 & $<2.2 \mathrm{e}-16$ & **** \\
\hline \multicolumn{8}{|c|}{ Mauchly Tests for Sphericity } \\
\hline & Test statistic & p-value & & & & & \\
\hline Scenario & $3.2246 \mathrm{e}-08$ & $2.1461 \mathrm{e}-22$ & & & & & \\
\hline \multicolumn{8}{|c|}{ Corrections for Departure from Sphericity } \\
\hline \multirow{3}{*}{ Scenario } & GG eps & $\operatorname{Pr}(>\mathrm{F}[\mathrm{GG}])$ & & & \multirow{3}{*}{\multicolumn{3}{|c|}{ Greenhouse-Geisser }} \\
\hline & 0.2532 & $4.989 \mathrm{e}-07$ & $* * *$ & & & & \\
\hline & H F eps & $\operatorname{Pr}(>\mathrm{F}[\mathrm{HF}])$ & & & & & \\
\hline Scenario & 0.2544114 & $4.712382 \mathrm{e}-07$ & $* * *$ & & Huynh-F & & \\
\hline
\end{tabular}

Table VIII-12. Repeated-measures ANOVA, with correction for sphericity, applied to five scenarios ( $N D n C, N D n C a, N D n C b, N D n C a b, N D n C c)$ of measurement uncertainty estimation in AGB calculation in El Salto, Durango, Mexico.

\begin{tabular}{c|ccccc}
\hline Scenario & Group (Holm correction) & NDnC & NDnCa & NDnCab & NDnCb \\
\hline$N D n C$ & $\mathrm{~A}$ & - & - & - & - \\
\hline$N D n C a$ & $\mathrm{~B}$ & $2.00 \mathrm{e}-06$ & - & - & - \\
\hline$N D n C b$ & $\mathrm{C}$ & $2.40 \mathrm{e}-06$ & 0.03509 & - & - \\
\hline$N D n C a b$ & $\mathrm{D}$ & $5.60 \mathrm{e}-06$ & 0.02048 & 0.00015 & - \\
\hline$N D n C c$ & $\mathrm{E}$ & 0.03509 & $3.70 \mathrm{e}-06$ & $4.10 \mathrm{e}-06$ & $9.80 \mathrm{e}-06$ \\
\hline
\end{tabular}

Table VIII-13. Pairwise comparison using paired T-Test, with p-value Holm's correction, for five scenarios ( $N D n C, N D n C a, N D n C b, N D n C a b, N D n C c)$ of AGB uncertainty estimation in El Salto, Durango, Mexico. 


\begin{tabular}{ll|llllllllll}
\hline Scenario & Plot & $\mathbf{1}$ & $\mathbf{2}$ & $\mathbf{3}$ & $\mathbf{4}$ & $\mathbf{5}$ & $\mathbf{6}$ & $\mathbf{7}$ & $\mathbf{8}$ & $\mathbf{9}$ & $\mathbf{1 0}$ \\
\hline$M C N D n$ & $\mathrm{uMg} \mathrm{ha}^{-1}$ & 3.69 & 4.94 & 5.91 & 6.28 & 2.89 & 4.00 & 3.85 & 5.19 & 5.15 & 4.59 \\
$M C N D n C$ & $\mathrm{uMg} \mathrm{ha}^{-1}$ & 3.67 & 5.02 & 6.01 & 6.26 & 3.25 & 4.11 & 3.86 & 5.25 & 5.12 & 4.63 \\
$M C B D$ & $\mathrm{uMg} \mathrm{ha}^{-1}$ & 3.71 & 4.99 & 5.95 & 6.42 & 3.08 & 4.00 & 3.95 & 5.20 & 5.17 & 4.60 \\
$M C B D C$ & $\mathrm{uMg} \mathrm{ha}^{-1}$ & 3.73 & 5.05 & 5.98 & 6.26 & 3.46 & 4.37 & 3.95 & 5.24 & 5.18 & 4.73 \\
$N D n$ & $\mathrm{uMg} \mathrm{ha}^{-1}$ & 3.74 & 5.05 & 6.10 & 6.40 & 3.00 & 4.10 & 3.93 & 5.26 & 5.26 & 4.73 \\
$N D n C$ & $\mathrm{uMg} \mathrm{ha}^{-1}$ & 3.79 & 5.07 & 6.10 & 6.41 & 3.31 & 4.26 & 4.04 & 5.29 & 5.26 & 4.77 \\
$R M S D$ & $\mathrm{uMg} \mathrm{ha}^{-1}$ & 3.79 & 5.09 & 6.12 & 6.43 & 3.13 & 4.17 & 3.99 & 5.30 & 5.27 & 4.77 \\
\hline \multicolumn{7}{l}{$\begin{array}{l}\text { For Monte-Carlo simulation Method: } M C N D n=\text { normal distribution, } M C N D n C=\text { normal distribution by class, } M C B D n=\text { best adjusted } \\
\text { distribution, } M C B D C=\text { best adjusted distribution by class. For } G U M\end{array}$} \\
class, $R M S D=$ Method: $N D n=$ normal distribution, $N D n C=$ normal distribution by
\end{tabular}

Table VIII-14. Uncertainty of the AGB calculation by plot using seven scenarios for the measurement uncertainty estimation ( $M C N D n, M C N D n C, M C B D, M C B D C, N D n, N D n C, R M S D)$ in El Salto, Durango, Mexico.

\begin{tabular}{|c|c|c|c|c|c|c|c|}
\hline & Df & Sum Sq & Error SS & den Df & F value & $\operatorname{Pr}(>\mathbf{F})$ & \\
\hline Intercept & 1 & 1580.52 & 65.186 & 9 & 218.2145 & $1.288 \mathrm{e}-07$ & $* * *$ \\
\hline Scenario & 6 & 0.25 & 0.296 & 54 & 7.5071 & $7.057 \mathrm{e}-06$ & $* * *$ \\
\hline \multicolumn{8}{|c|}{ Mauchly Tests for Sphericity } \\
\hline & Test statistic & p-value & & & & & \\
\hline Scenario & $1.0916 \mathrm{e}-05$ & $4.3153 \mathrm{e}-08$ & & & & & \\
\hline \multicolumn{8}{|c|}{ Corrections for Departure from Sphericity } \\
\hline \multirow{3}{*}{ Scenario } & GG eps & $\operatorname{Pr}(>\mathrm{F}[\mathrm{GG}])$ & & & \multirow{3}{*}{\multicolumn{3}{|c|}{ Greenhouse-Geisser }} \\
\hline & 0.23674 & 0.0112 & * & & & & \\
\hline & H F eps & $\operatorname{Pr}(>\mathrm{F}[\mathrm{HF}])$ & & & & & \\
\hline Scenario & 0.2683571 & 0.008144593 & $* *$ & & Huynh-Fe & & \\
\hline
\end{tabular}

Table VIII-15. Repeated-measures ANOVA with correction for sphericity applied to seven scenarios ( $M C N D n, M C N D n C, M C B D, M C B D C, N D n, N D n C, R M S D$ ) of measurement uncertainty estimation in AGB calculation in El Salto, Durango.

\begin{tabular}{lcrrrrrr}
\hline Scenario & $\begin{array}{l}\text { Group } \\
\text { (Holm } \\
\text { correction) }\end{array}$ & MCBD & MCBDC & MCNDn & MCNDnC & NDn & NDnC \\
\hline$M C B D$ & $\mathrm{AB}$ & & & & & & \\
$M C B D C$ & $\mathrm{ABCD}$ & 1 & - & - & - & - & - \\
$M C N D n$ & $\mathrm{~A}$ & 0.39802 & 0.68129 & & - & - & - \\
$M C N D n C$ & $\mathrm{ABD}$ & 1 & 0.61451 & 1 & - & - & - \\
$N D n$ & $\mathrm{BC}$ & 1 & 1 & 0.00022 & 1 & - & - \\
$N D n C$ & $\mathrm{CD}$ & 0.01899 & 1 & 0.00528 & 0.00099 & 0.89258 & - \\
$R M S D$ & $\mathrm{D}$ & 0.00811 & 1 & $2.90 \mathrm{e}-05$ & 0.18097 & 0.02605 & 1 \\
\hline
\end{tabular}

Table VIII-16. Pairwise comparison using paired T-Test, with p-value Holm's correction, for seven scenarios (MCNDn, $M C N D n C, M C B D, M C B D C, N D n, N D n C, R M S D)$ of AGB uncertainty estimation in El Salto, Durango Mexico. 


\begin{tabular}{ll|llllllllll}
\hline Scenario & Plot & $\mathbf{1}$ & $\mathbf{2}$ & $\mathbf{3}$ & $\mathbf{4}$ & $\mathbf{5}$ & $\mathbf{6}$ & $\mathbf{7}$ & $\mathbf{8}$ & $\mathbf{9}$ & $\mathbf{1 0}$ \\
\hline$M C C$ & $\mathrm{uMg} \mathrm{ha}^{-1}$ & 3.73 & 5.05 & 5.98 & 6.26 & 3.46 & 4.37 & 3.95 & 5.24 & 5.18 & 4.73 \\
$M C C a$ & $\mathrm{uMg} \mathrm{ha}^{-1}$ & 0.54 & 0.52 & 0.39 & 0.53 & 1.39 & 1.09 & 0.78 & 0.61 & 0.26 & 0.60 \\
$M C C b$ & $\mathrm{uMg} \mathrm{ha}^{-1}$ & 0.57 & 0.51 & 0.38 & 0.52 & 1.34 & 1.12 & 0.76 & 0.63 & 0.26 & 0.59 \\
$M C C C$ & $\mathrm{uMg} \mathrm{ha}^{-1}$ & 3.68 & 4.92 & 6.01 & 6.15 & 2.88 & 3.97 & 3.76 & 5.11 & 5.16 & 4.60 \\
\multicolumn{7}{l}{$M C C=$ all sources, $M C C a=D B H, M C C b=T H, M C C C=$ prediction model }
\end{tabular}

Table VIII-17. AGB uncertainty estimation by plot with Monte-Carlo simulation Method using non-sampling uncertainty estimation sources $(M C C, M C C a, M C C b, M C C c)$ in El Salto, Durango, Mexico.

\begin{tabular}{|c|c|c|c|c|c|c|c|}
\hline & Df & Sum Sq & Error SS & den Df & F value & $\operatorname{Pr}(>\mathbf{F})$ & \\
\hline Intercept & 1 & 289.34 & 5.5755 & 9 & 467.05 & $4.58 \mathrm{e}-09$ & $* * *$ \\
\hline Scenario & 3 & 163.36 & 14.1684 & 27 & 103.77 & $6.17 \mathrm{e}-15$ & $* * *$ \\
\hline \multicolumn{8}{|c|}{ Mauchly Tests for Sphericity } \\
\hline Scenario & $\begin{array}{r}\text { Test statistic } \\
1.7331 \mathrm{e}-05 \\
\end{array}$ & $\begin{array}{l}\mathrm{p} \text {-value } \\
\quad 2.3872 \mathrm{e}-16\end{array}$ & & & & & \\
\hline \multicolumn{8}{|c|}{ Corrections for Departure from Sphericity } \\
\hline & GG eps & $\operatorname{Pr}(>\mathrm{F}[\mathrm{GG}])$ & & & \multirow{2}{*}{\multicolumn{3}{|c|}{ Greenhouse-Geisser }} \\
\hline Scenario & $\begin{array}{l}0.33618 \\
\text { H F eps }\end{array}$ & $\begin{array}{c}2.813 \mathrm{e}-06 \\
\operatorname{Pr}(>\mathrm{F}[\mathrm{HF}])\end{array}$ & $* * *$ & & & & \\
\hline Scenario & 0.3372499 & $2.722362 \mathrm{e}-06$ & $* * *$ & & Huynh-F & & \\
\hline
\end{tabular}

Table VIII-18. Repeated-measures ANOVA with correction for sphericity applied to four scenarios $(M C C, M C C a, M C C b, M C C c$ ) of AGB uncertainty estimation in El Salto, Durango.

\begin{tabular}{c|cccc}
\hline Scenario & Group (Holm correction) & $\boldsymbol{M C C}$ & $\boldsymbol{M C C} \boldsymbol{a}$ & $\boldsymbol{M C C b}$ \\
\hline$M C C$ & $\mathrm{~A}$ & - & - & - \\
\hline$M C C a$ & $\mathrm{~B}$ & $8.9 \mathrm{e}-06$ & - & - \\
\hline$M C C b$ & $\mathrm{~B}$ & $8.9 \mathrm{e}-06$ & 0.708 & - \\
\hline$M C C \mathrm{C}$ & $\mathrm{C}$ & 0.034 & $2.2 \mathrm{e}-05$ & $2.2 \mathrm{e}-05$ \\
\hline
\end{tabular}

Table VIII-19. Pairwise comparison using paired T-Test, with p-value Holm's correction, for four scenarios ( $M C C, M C C a, M C C b, M C C c$ ) of AGB uncertainty estimation in El Salto, Durango Mexico. 


\section{VIII.5 Appendix V}

\begin{tabular}{cc|ccccccccccc}
\hline Scenario & Plot & $\mathbf{1}$ & $\mathbf{2}$ & $\mathbf{3}$ & $\mathbf{4}$ & $\mathbf{5}$ & $\mathbf{6}$ & $\mathbf{7}$ & $\mathbf{8}$ & $\mathbf{9}$ & $\mathbf{1 0}$ & $\mathbf{1 1}$ \\
\hline \multirow{2}{*}{$C B$} & $\mathrm{Mg} \mathrm{ha}^{-1}$ & 234.69 & 225.00 & 314.25 & 213.68 & 238.98 & 206.33 & 233.74 & 250.95 & 159.27 & 207.77 & 314.40 \\
$C$ & $\mathrm{Mg} \mathrm{ha}^{-1}$ & 224.11 & 209.17 & 313.39 & 197.77 & 214.43 & 202.53 & 205.08 & 242.94 & 144.64 & 196.40 & 306.98 \\
$T V$ & $\mathrm{Mg} \mathrm{ha}^{-1}$ & 238.94 & 236.77 & 325.09 & 218.01 & 230.89 & 210.65 & 234.78 & 252.99 & 154.77 & 206.96 & 315.19 \\
$T$ & $\mathrm{Mg} \mathrm{ha}^{-1}$ & 231.77 & 213.93 & 315.47 & 202.20 & 218.89 & 206.16 & 211.06 & 254.55 & 146.92 & 199.91 & 314.15 \\
\hline \multicolumn{2}{l}{ C=Caliper, B=Blume-Leiss, T=Tape, V=Vertex }
\end{tabular}

Table VIII-20. AGB calculation by Plot using three scenarios for the measurement uncertainty estimation $(C B, C, T V, T)$ in Göttingen, Germany.

\begin{tabular}{|c|c|c|c|c|c|c|c|}
\hline & Df & Sum Sq & Error SS & den Df & F value & $\operatorname{Pr}(>\mathbf{F})$ & \\
\hline Intercept & 1 & 1528.14 & 2.3268 & 10 & 6567.517 & $2.000 \mathrm{e}-15$ & $* * *$ \\
\hline Scenario & 3 & 0.05 & 0.0231 & 30 & 20.532 & $1.995 \mathrm{e}-07$ & $* * *$ \\
\hline \multicolumn{8}{|c|}{ Mauchly Tests for Sphericity } \\
\hline Scenario & $\begin{array}{r}\text { Test statistic } \\
0.12534 \\
\end{array}$ & $\begin{array}{l}\mathrm{p} \text {-value } \\
0.0029995\end{array}$ & & & & & \\
\hline \multicolumn{8}{|c|}{ Corrections for Departure from Sphericity } \\
\hline & GG eps & $\operatorname{Pr}(>\mathrm{F}[\mathrm{GG}])$ & & & \multirow{2}{*}{\multicolumn{3}{|c|}{ Greenhouse-Geisser }} \\
\hline Scenario & $\begin{array}{l}0.50987 \\
\text { H F eps }\end{array}$ & $\begin{aligned} & 0.000108 \\
& \operatorname{Pr}(>F[H F])\end{aligned}$ & $* * *$ & & & & \\
\hline Scenario & 0.5834365 & $4.159047 \mathrm{e}-05$ & $* * *$ & & Huynh-Fe & & \\
\hline
\end{tabular}

Table VIII-21. Repeated-measures ANOVA with correction for sphericity applied to four scenarios $(C B, C, T V, T)$ of AGB estimation in Göttingen, Germany.

\begin{tabular}{c|cccc}
\hline Scenario & Group (Holm correction) & $\boldsymbol{C}$ & $\boldsymbol{C B}$ & $\boldsymbol{T}$ \\
\hline$C$ & $\mathrm{~A}$ & - & - & - \\
$C B$ & $\mathrm{~B}$ & 0.0023 & - & - \\
$T$ & $\mathrm{C}$ & 0.0002 & 0.0256 & - \\
$T V$ & $\mathrm{~B}$ & 0.0004 & 0.2916 & 0.0061 \\
\hline
\end{tabular}

Table VIII-22. Pairwise comparison using paired T-Test, with p-value Holm's correction, for three scenarios $(C B, C, T V, T)$ of AGB estimation in Göttingen, Germany. 


\begin{tabular}{cc|ccccccccccc}
\hline Scenario & Plot & $\mathbf{1}$ & $\mathbf{2}$ & $\mathbf{3}$ & $\mathbf{4}$ & $\mathbf{5}$ & $\mathbf{6}$ & $\mathbf{7}$ & $\mathbf{8}$ & $\mathbf{9}$ & $\mathbf{1 0}$ & $\mathbf{1 1}$ \\
\hline$C B$ & $\mathrm{Mg} \mathrm{ha}^{-1}$ & 1.83 & 3.03 & 4.67 & 2.36 & 3.78 & 2.57 & 2.13 & 3.62 & 1.88 & 3.08 & 3.47 \\
$C$ & $\mathrm{Mg} \mathrm{ha}^{-1}$ & 1.62 & 1.97 & 2.54 & 1.77 & 2.30 & 1.80 & 1.50 & 3.49 & 1.06 & 2.20 & 2.75 \\
$T V$ & $\mathrm{Mg} \mathrm{ha}^{-1}$ & 1.02 & 1.67 & 3.04 & 1.89 & 1.94 & 1.69 & 2.00 & 2.34 & 1.50 & 1.45 & 2.70 \\
$T$ & $\mathrm{Mg} \mathrm{ha}^{-1}$ & 0.93 & 1.46 & 1.66 & 0.83 & 1.43 & 1.34 & 1.12 & 1.09 & 0.91 & 1.22 & 1.30 \\
\multicolumn{2}{l}{$C=$ Caliper, $B=$ Blume-Leiss, $T=$ Tape, $V=$ Vertex }
\end{tabular}

Table VIII-23. Uncertainty of the AGB calculation by plot using four scenarios for the measurement uncertainty estimation $(C B, C, T V, T)$ in Göttingen, Germany.

\begin{tabular}{|c|c|c|c|c|c|c|c|}
\hline & Df & Sum Sq & Error SS & den Df & F value & $\operatorname{Pr}(>\mathbf{F})$ & \\
\hline Intercept & 1 & 14.9457 & 2.01731 & 10 & 74.087 & $6.168 \mathrm{e}-06$ & $* * *$ \\
\hline Scenario & 3 & 3.4482 & 0.70439 & 30 & 48.953 & $1.137 \mathrm{e}-11$ & $* * *$ \\
\hline \multicolumn{8}{|c|}{ Mauchly Tests for Sphericity } \\
\hline Scenario & $\begin{array}{r}\text { Test statistic } \\
0.38221\end{array}$ & $\begin{array}{l}\mathrm{p} \text {-value } \\
0.13829\end{array}$ & & & & & \\
\hline \multicolumn{8}{|c|}{ Corrections for Departure from Sphericity } \\
\hline & GG eps & $\operatorname{Pr}(>\mathrm{F}[\mathrm{GG}])$ & & & \multirow{2}{*}{\multicolumn{3}{|c|}{ Greenhouse-Geisser }} \\
\hline Scenario & $\begin{array}{l}0.75414 \\
\text { H F eps }\end{array}$ & $\begin{array}{r}2.77 \mathrm{e}-09 \\
\operatorname{Pr}(>\mathrm{F}[\mathrm{HF}])\end{array}$ & $* * *$ & & & & \\
\hline Scenario & 0.9859425 & $1.55613 \mathrm{e}-11$ & $* * *$ & & Huynh- & ldt & \\
\hline
\end{tabular}

Table VIII-24. Repeated-measures ANOVA with correction for sphericity applied to four scenarios $(C B, C, T V, T)$ of AGB uncertainty estimation in Göttingen, Germany.

\begin{tabular}{c|cccc}
\hline Scenario & Group (Holm correction) & $\boldsymbol{C}$ & $\boldsymbol{C B}$ & $\boldsymbol{T}$ \\
\hline$C$ & $\mathrm{~A}$ & - & - & - \\
$C B$ & $\mathrm{~B}$ & 0.00034 & - & - \\
$T$ & $\mathrm{C}$ & 0.00034 & $1.1 \mathrm{e}-07$ & - \\
$T V$ & $\mathrm{~A}$ & 0.41637 & 0.00034 & 0.00051 \\
\hline
\end{tabular}

Table VIII-25. Pairwise comparison using paired T-Test, with p-value Holm's correction, for four scenarios $(C B, C, T V, T)$ AGB uncertainty estimation in Göttingen, Germany. 


\begin{tabular}{|c|c|c|c|c|c|c|c|c|c|c|c|c|}
\hline Scenario & Plot & 1 & 2 & 3 & 4 & 5 & 6 & 7 & 8 & 9 & 10 & 11 \\
\hline AGB & $\mathrm{Mg} \mathrm{ha}^{-1}$ & 234.69 & 225.00 & 314.25 & 213.68 & 238.98 & 206.33 & 233.74 & 250.95 & 159.27 & 207.77 & 314.40 \\
\hline$C B$ & uMgha $^{-1}$ & 1.83 & 3.03 & 4.67 & 2.36 & 3.78 & 2.57 & 2.13 & 3.62 & 1.88 & 3.08 & 3.47 \\
\hline CBmean & uMgha $^{-1}$ & 1.63 & 2.21 & 2.86 & 1.93 & 2.33 & 2.09 & 2.14 & 2.66 & 1.52 & 2.37 & 2.62 \\
\hline CBNDn & uMgha $^{-1}$ & 2.13 & 2.94 & 3.83 & 2.56 & 3.10 & 2.78 & 2.84 & 3.59 & 2.00 & 3.20 & 3.49 \\
\hline$C B N D n C$ & uMgha $^{-1}$ & 2.55 & 3.52 & 4.57 & 3.07 & 3.78 & 3.28 & 3.38 & 4.33 & 2.22 & 3.87 & 4.13 \\
\hline CBmod & uMgha $^{-1}$ & 1.85 & 2.62 & 3.39 & 2.29 & 2.89 & 2.32 & 2.58 & 3.35 & 1.59 & 3.10 & 3.00 \\
\hline
\end{tabular}

Table VIII-26. AGB and uncertainty of the AGB calculation by plot using five scenarios for measurement uncertainty estimation $(C B, C B m e a n, C B N D n, C B N D n C, C B m o d)$ in Göttingen, Germany.

\begin{tabular}{|c|c|c|c|c|c|c|c|}
\hline & Df & Sum Sq & Error SS & den Df & F value & $\operatorname{Pr}(>\mathbf{F})$ & \\
\hline Intercept & 1 & 569.60 & 23.4236 & 10 & 243.172 & $2.406 \mathrm{e}-08$ & $* * *$ \\
\hline Scenario & 3 & 12.44 & 2.2049 & 40 & 56.433 & $<2.2 \mathrm{e}-16$ & $* * *$ \\
\hline \multicolumn{8}{|c|}{ Mauchly Tests for Sphericity } \\
\hline & Test statistic & p-value & & & & & \\
\hline Scenario & 0.000412 & $7.7684 \mathrm{e}-08$ & & & & & \\
\hline \multicolumn{8}{|c|}{ Corrections for Departure from Sphericity } \\
\hline & GG eps & $\operatorname{Pr}(>\mathrm{F}[\mathrm{GG}])$ & & & & & \\
\hline Scenario & $\begin{array}{l}0.31273 \\
\text { H F eps }\end{array}$ & $\begin{array}{r}2.045 \mathrm{e}-07 \\
\operatorname{Pr}(>\mathrm{F}[\mathrm{HF}])\end{array}$ & $* * *$ & & \multicolumn{3}{|c|}{ Greenhouse-Geisser } \\
\hline Scenario & 0.3603441 & $2.9679 \mathrm{e}-08$ & $* * *$ & & \multicolumn{3}{|c|}{ Huynh-Feldt } \\
\hline
\end{tabular}

Table VIII-27. Repeated-measures ANOVA with correction for sphericity applied to five scenarios $(C B, C B m e a n, C B N D n, C B N D n C, C B m o d)$ of AGB uncertainty estimation in Göttingen, Germany.

\begin{tabular}{lccccc}
\hline \multicolumn{1}{c}{ Scenario } & Group (Holm correction) & $\boldsymbol{C B}$ & CBmean & CBmod & CBNDn \\
\hline$C B$ & AC & & & & \\
CBmean & B & 0.00546 & - & - & - \\
CBmod & A & 0.10039 & 0.00029 & - & - \\
$C B N D n$ & C & 0.97838 & $3.9 \mathrm{e}-07$ & $4.5 \mathrm{e}-05$ & - \\
$C B N D n C$ & D & 0.00322 & $7.6 \mathrm{e}-07$ & $1.5 \mathrm{e}-07$ & $2.3 \mathrm{e}-06$ \\
\hline
\end{tabular}

Table VIII-28. Pairwise comparison using paired T-Test, with p-value Holm's correction, for five scenarios ( $C B, C B m e a n, C B N D n, C B N D n C, C B m o d)$ of AGB uncertainty estimation in El Salto, Durango Mexico. 


\begin{tabular}{|c|c|c|c|c|c|c|c|c|c|c|c|c|}
\hline Scenario & Plot & 1 & 2 & 3 & 4 & 5 & 6 & 7 & 8 & 9 & 10 & 11 \\
\hline AGB & $\mathrm{Mg} \mathrm{ha}^{-1}$ & 238.94 & 236.77 & 325.09 & 218.01 & 230.89 & 210.65 & 234.78 & 252.99 & 154.77 & 206.96 & 315.19 \\
\hline$T V$ & uMgha $^{-1}$ & 1.02 & 1.67 & 3.04 & 1.89 & 1.94 & 1.69 & 2.00 & 2.34 & 1.50 & 1.45 & 2.70 \\
\hline TVmean & uMgha $^{-1}$ & 1.20 & 1.57 & 2.00 & 1.38 & 1.66 & 1.48 & 1.54 & 1.91 & 1.09 & 1.65 & 1.87 \\
\hline TVNDn & uMgha $^{-1}$ & 1.94 & 2.54 & 3.23 & 2.24 & 2.68 & 2.38 & 2.50 & 3.09 & 1.76 & 2.66 & 3.02 \\
\hline TVNDnC & $\mathrm{uMgha}^{-1}$ & 1.96 & 2.62 & 3.30 & 2.28 & 2.77 & 2.36 & 2.55 & 3.05 & 1.63 & 2.69 & 3.03 \\
\hline TVmod & uMgha $^{-1}$ & 1.54 & 2.17 & 2.75 & 1.87 & 2.27 & 1.84 & 2.10 & 2.63 & 1.23 & 2.38 & 2.37 \\
\hline
\end{tabular}

Table VIII-29. Uncertainty of the AGB calculation by plot using six scenarios for the measurement uncertainty estimation (TV, TVmean, TVNDn, TVNDnC, TVmod) in Göttingen, Germany.

\begin{tabular}{|c|c|c|c|c|c|c|c|}
\hline & Df & Sum Sq & Error SS & den Df & F value & $\operatorname{Pr}(>\mathbf{F})$ & \\
\hline Intercept & 1 & 289.047 & 10.4404 & 10 & 276.855 & $1.286 \mathrm{e}-08$ & $* * *$ \\
\hline Scenario & 3 & 8.755 & 1.4197 & 40 & 61.669 & $<2.2 \mathrm{e}-16$ & $* * *$ \\
\hline \multicolumn{8}{|c|}{ Mauchly Tests for Sphericity } \\
\hline Scenario & $\begin{array}{r}\text { Test statistic } \\
3.8811 \mathrm{e}-06 \\
\end{array}$ & $\begin{array}{l}\mathrm{p} \text {-value } \\
1.4135 \mathrm{e}-14\end{array}$ & & & & & \\
\hline \multicolumn{8}{|c|}{ Corrections for Departure from Sphericity } \\
\hline & GG eps & $\operatorname{Pr}(>\mathrm{F}[\mathrm{GG}])$ & & & \multirow{2}{*}{\multicolumn{3}{|c|}{ Greenhouse-Geisser }} \\
\hline Scenario & $\begin{array}{l}0.33879 \\
\text { H F eps }\end{array}$ & $\begin{array}{r}3.73 \mathrm{e}-08 \\
\operatorname{Pr}(>\mathrm{F}[\mathrm{HF}])\end{array}$ & $* * *$ & & & & \\
\hline Scenario & 0.4005151 & $2.7366 \mathrm{e}-09$ & $* * *$ & & Huynh-F & & \\
\hline
\end{tabular}

Table VIII-30. Repeated-measures ANOVA with correction for sphericity applied to five scenarios (TV, TVmean, TVNDn, TVNDnC, TVmod) of AGB uncertainty estimation in Göttingen, Germany.

\begin{tabular}{lccccc}
\hline \multicolumn{1}{c}{ Scenario } & $\begin{array}{c}\text { Group (Holm } \\
\text { correction) }\end{array}$ & $\boldsymbol{T V}$ & TVmean & TVmod & TVNDn \\
\hline$T V$ & AC & - & - & - & - \\
TVmean & B & 0.04442 & - & - & - \\
$T V$ mod & $\mathrm{A}$ & 0.52100 & $2.50 \mathrm{e}-05$ & - & - \\
$T V N D n$ & $\mathrm{C}$ & 0.71675 & $1.80 \mathrm{e}-08$ & 0.00081 & - \\
$T V N D n C$ & $\mathrm{D}$ & 0.00061 & $2.70 \mathrm{e}-07$ & $1.60 \mathrm{e}-07$ & $5.30 \mathrm{e}-07$ \\
\hline
\end{tabular}

Table VIII-31. Pairwise comparison using paired T-Test, with p-value Holm's correction, for seven scenarios (TV, TVmean, TVNDn, TVNDnC, TVmod) of AGB uncertainty estimation in Göttingen, Germany. 


\begin{tabular}{|c|c|c|c|c|c|c|c|c|c|c|c|c|}
\hline Scenario & Plot & 1 & 2 & 3 & 4 & 5 & 6 & 7 & 8 & 9 & 10 & 11 \\
\hline TVExp & $\mathrm{uMg} \mathrm{ha}^{-1}$ & 8.22 & 10.82 & 13.74 & 9.52 & 11.41 & 10.14 & 10.62 & 13.17 & 7.49 & 11.36 & 12.82 \\
\hline TVNexp & $\mathrm{uMg} \mathrm{ha}^{-1}$ & 8.30 & 10.93 & 13.88 & 9.61 & 11.52 & 10.23 & 10.72 & 13.31 & 7.56 & 11.47 & 12.95 \\
\hline$T V$ & $\mathrm{uMg} \mathrm{ha} \mathrm{h}^{-1}$ & 1.02 & 1.67 & 3.04 & 1.89 & 1.94 & 1.69 & 2.00 & 2.34 & 1.50 & 1.45 & 2.70 \\
\hline
\end{tabular}

Table VIII-32. Uncertainty of the AGB calculation by plot using three scenarios for the measurement uncertainty estimation (TV, TVExp, TVNexp) in Göttingen, Germany.

\begin{tabular}{|c|c|c|c|c|c|c|c|}
\hline & Df & Sum Sq & Error SS & den Df & F value & $\operatorname{Pr}(>\mathbf{F})$ & \\
\hline Intercept & 1 & 208.232 & 3.7645 & 10 & 553.14 & $4.378 \mathrm{e}-10$ & **** \\
\hline Scenario & $\underline{3}$ & 54.793 & 0.3438 & 20 & 1593.69 & $<2.2 \mathrm{e}-16$ & **** \\
\hline \multicolumn{8}{|c|}{ Mauchly Tests for Sphericity } \\
\hline Scenario & $\begin{array}{r}\text { Test statistic } \\
0.00017479 \\
\end{array}$ & $\begin{array}{l}\mathrm{p} \text {-value } \\
1.2341 \mathrm{e}-17\end{array}$ & & & & & \\
\hline \multicolumn{8}{|c|}{ Corrections for Departure from Sphericity } \\
\hline & GG eps & $\operatorname{Pr}(>\mathrm{F}[\mathrm{GG}])$ & & & \multirow{2}{*}{\multicolumn{3}{|c|}{ Greenhouse-Geisser }} \\
\hline Scenario & $\begin{array}{l}0.50004 \\
\text { H F eps }\end{array}$ & $\begin{array}{c}2.321 \mathrm{e}-12 \\
\operatorname{Pr}(>\mathrm{F}[\mathrm{HF}])\end{array}$ & $* * *$ & & & & \\
\hline Scenario & 0.5000583 & $2.319561 \mathrm{e}-12$ & $* * *$ & & Huynh-F & & \\
\hline
\end{tabular}

Table VIII-33. Repeated-measures ANOVA with correction for sphericity applied to three scenarios (TV, TVExp, TVNexp) of AGB uncertainty estimation in Göttingen, Germany.

\begin{tabular}{lccc}
\hline Scenario & Group (Holm correction) & TVExp & TVNesp \\
\hline TVExp & A & - & - \\
TVNexp & B & $9.2 \mathrm{e}-12$ & - \\
$T V$ & C & $6.8 \mathrm{e}-12$ & $6.8 \mathrm{e}-12$ \\
\hline
\end{tabular}

Table VIII-34. Pairwise comparison using paired T-Test, with p-value Holm's correction, for three scenarios (TV, TVExp, TVNexp) of AGB uncertainty estimation in Göttingen, Germany. 


\begin{tabular}{|c|c|c|c|c|c|c|c|}
\hline & Df & Sum Sq & Error SS & den Df & F value & $\operatorname{Pr}(>\mathbf{F})$ & \\
\hline Intercept & 1 & 491.65 & 154.465 & 46 & 146.41 & $6.772 \mathrm{e}-16$ & **** \\
\hline Scenario & 3 & 16.70 & 4.425 & 138 & 173.60 & $<2.2 \mathrm{e}-16$ & **** \\
\hline \multicolumn{8}{|c|}{ Mauchly Tests for Sphericity } \\
\hline & Test statistic & p-value & & & & & \\
\hline Scenario & 0.023595 & $2.884 \mathrm{e}-34$ & & & & & \\
\hline \multicolumn{8}{|c|}{ Corrections for Departure from Sphericity } \\
\hline \multirow{3}{*}{ Scenario } & GG eps & $\operatorname{Pr}(>\mathrm{F}[\mathrm{GG}])$ & & & \multirow{3}{*}{\multicolumn{3}{|c|}{ Greenhouse-Geisser }} \\
\hline & 0.39652 & $2.2 \mathrm{e}-16$ & $* * *$ & & & & \\
\hline & H F eps & $\operatorname{Pr}(>\mathrm{F}[\mathrm{HF}])$ & & & & & \\
\hline Scenario & 0.4010133 & $3.207957 \mathrm{e}-20$ & $* * *$ & & Huynh-F & & \\
\hline
\end{tabular}

Table VIII-35. Repeated-measures ANOVA with correction for sphericity applied to three scenarios ( $C B B D, C B B D C, C B m o d, C B N D n)$ of AGB uncertainty estimation in Göttingen, Germany.

\begin{tabular}{lcccc}
\hline Scenario & Group (Holm correction) & $\boldsymbol{C B B D}$ & $\boldsymbol{C B B D C}$ & $\boldsymbol{C B m o d}$ \\
\hline$C B B D$ & $\mathrm{~A}$ & - & - & - \\
\hline$C B B D C$ & $\mathrm{~B}$ & $<2 \mathrm{e}-16$ & - & - \\
$C B m o d$ & $\mathrm{C}$ & $4.7 \mathrm{e}-10$ & $<2 \mathrm{e}-16$ & - \\
$C B N D n$ & $\mathrm{D}$ & $<2 \mathrm{e}-16$ & $7.5 \mathrm{e}-14$ & $3.2 \mathrm{e}-05$ \\
\hline
\end{tabular}

Table VIII-36. Pairwise comparison using paired T-Test, with p-value Holm's correction, for four scenarios $(C B B D, C B B D C, C B m o d, C B N D n)$ of AGB uncertainty estimation in Göttingen, Germany.

\begin{tabular}{|c|c|c|c|c|c|c|c|}
\hline & Df & Sum Sq & Error SS & den Df & F value & $\operatorname{Pr}(>\mathbf{F})$ & \\
\hline Intercept & 1 & 96.174 & 63.693 & 46 & 69.458 & $9.559 \mathrm{e}-11$ & **** \\
\hline Scenario & 3 & 5.262 & 2.746 & 138 & 88.130 & $<2.2 \mathrm{e}-16$ & $* * *$ \\
\hline \multicolumn{8}{|c|}{ Mauchly Tests for Sphericity } \\
\hline & Test statistic & p-value & & & & & \\
\hline Scenario & 0.0062212 & $5.8209 \mathrm{e}-47$ & & & & & \\
\hline \multicolumn{8}{|c|}{ Corrections for Departure from Sphericity } \\
\hline \multirow{3}{*}{ Scenario } & GG eps & $\operatorname{Pr}(>\mathrm{F}[\mathrm{GG}])$ & & & \multirow{3}{*}{\multicolumn{3}{|c|}{ Greenhouse-Geisser }} \\
\hline & 0.37587 & $1.603 \mathrm{e}-13$ & $* * *$ & & & & \\
\hline & H F eps & $\operatorname{Pr}(>\mathrm{F}[\mathrm{HF}])$ & & & & & \\
\hline Scenario & 0.37884 & $1.309963 \mathrm{e}-13$ & $* * *$ & & Huynh-F & & \\
\hline
\end{tabular}

Table VIII-37. Repeated-measures ANOVA with correction for sphericity applied to four scenarios (TVBPDF, TVBDC, TVmod, TVNDn) of AGB uncertainty estimation in Göttingen, Germany.

\begin{tabular}{lcccc}
\hline Scenario & Group (Holm correction) & TVBPDF & TVBDC & TVmod \\
\hline$T V B P D F$ & A & - & - & - \\
$T V B D C$ & B & $6.3 \mathrm{e}-16$ & - & - \\
$T V m o d$ & C & $1.2 \mathrm{e}-08$ & $4.1 \mathrm{e}-08$ & - \\
$T V N D n$ & D & $7.0 \mathrm{e}-12$ & $6.3 \mathrm{e}-16$ & $4.1 \mathrm{e}-08$ \\
\hline
\end{tabular}

Table VIII-38. Pairwise comparison using paired T-Test, with p-value Holm's correction, for four scenarios (TVBPDF, TVBDC, TVmod, TVNDn) of AGB uncertainty estimation in Göttingen, Germany. 


\begin{tabular}{|c|c|c|c|c|c|c|c|}
\hline & Df & Sum Sq & Error SS & den Df & F value & $\operatorname{Pr}(>\mathbf{F})$ & \\
\hline Intercept & 1 & 621.88 & 82.216 & 46 & 347.94 & $<2.2 \mathrm{e}-16$ & $* * *$ \\
\hline Scenario & 5 & 283.31 & 2.006 & 230 & 6497.83 & $<2.2 \mathrm{e}-16$ & $* * *$ \\
\hline \multicolumn{8}{|c|}{ Mauchly Tests for Sphericity } \\
\hline Scenario & $\begin{array}{r}\text { Test statistic } \\
6.4315 \mathrm{e}-05 \\
\end{array}$ & $\begin{array}{l}\text { p-value } \\
1.5186 \mathrm{e}-81 \\
\end{array}$ & & & & & \\
\hline \multicolumn{8}{|c|}{ Corrections for Departure from Sphericity } \\
\hline & GG eps & $\operatorname{Pr}(>\mathrm{F}[\mathrm{GG}])$ & & & \multirow{2}{*}{\multicolumn{3}{|c|}{ Greenhouse-Geisser }} \\
\hline Scenario & $\begin{array}{l}0.26836 \\
\text { H F eps }\end{array}$ & $\begin{array}{c}\quad<2.2 \mathrm{e}-16 \\
\operatorname{Pr}(>\mathrm{F}[\mathrm{HF}])\end{array}$ & $* * *$ & & & & \\
\hline Scenario & 0.2734762 & $4.897372 \mathrm{e}-69$ & $* * *$ & & Huynh-F & & \\
\hline
\end{tabular}

Table VIII-39. Repeated-measures ANOVA with correction for sphericity applied to six scenarios (Exp, Nexp, CBmod, CBNDn, TVmod, TVNDn) of AGB uncertainty estimation in Göttingen, Germany.

\begin{tabular}{lcccccc}
\hline Scenario & Group (Holm correction) & CBmod & CBNDn & Exp & NExp & TVmod \\
\hline CBmod & A & - & - & - & - & - \\
CBNDn & B & $5.40 \mathrm{e}-05$ & - & - & - & - \\
Exp & C & $<2 \mathrm{e}-16$ & $<2 \mathrm{e}-16$ & - & - & - \\
Nexp & C & $<2 \mathrm{e}-16$ & $<2 \mathrm{e}-16$ & 0.99 & - & - \\
TVmod & $\mathrm{D}$ & $<2 \mathrm{e}-16$ & $<2 \mathrm{e}-16$ & $<2 \mathrm{e}-16$ & $<2 \mathrm{e}-16$ & - \\
TVNDn & $\mathrm{E}$ & $<2 \mathrm{e}-16$ & $<2 \mathrm{e}-16$ & $<2 \mathrm{e}-16$ & $<2 \mathrm{e}-16$ & $7.20 \mathrm{e}-06$ \\
\hline
\end{tabular}

Table VIII-40. Pairwise comparison using paired T-Test, with p-value Holm's correction, for six scenarios (Exp, Nexp, CBmod, CBNDn, TVmod, TVNDn) of AGB uncertainty estimation in Göttingen, Germany. 


\begin{tabular}{|c|c|c|c|c|c|c|c|}
\hline Uncertainty (u) & $u^{2}$ & $S E^{2}$ & $u$ & $W$ & RSE LL & RSE & RSE UL \\
\hline source & $\%$ & $\%$ & Mg ha' & Mg ha' & $\%$ & $\%$ & $\%$ \\
\hline$C B B D$ & 0.072 & 99.928 & 13.14 & 262.69 & 4.97 & 5.00 & 5.03 \\
\hline$C B B D$ a & 0.010 & 99.990 & 13.13 & 262.74 & 4.99 & 5.00 & 5.01 \\
\hline$C B B D \mathrm{~b}$ & 0.060 & 99.940 & 13.14 & 262.69 & 4.98 & 5.00 & 5.03 \\
\hline$C B B D \mathrm{ab}$ & 0.00002 & 100.00 & 13.13 & 262.71 & 5.00 & 5.00 & 5.00 \\
\hline$C B B D \mathrm{c}$ & 0.0002 & 100.00 & 13.13 & 262.71 & 5.00 & 5.00 & 5.00 \\
\hline CBmod & 0.100 & 99.900 & 13.14 & 262.70 & 4.97 & 5.00 & 5.03 \\
\hline CBmod a & 0.020 & 99.980 & 13.13 & 262.72 & 4.99 & 5.00 & 5.00 \\
\hline CBmod b & 0.080 & 99.920 & 13.14 & 262.68 & 4.99 & 5.00 & 5.01 \\
\hline CBmod ab & 0.00002 & 100.00 & 13.13 & 262.71 & 5.00 & 5.00 & 5.00 \\
\hline CBmod c & 0.0002 & 100.00 & 13.13 & 262.71 & 5.00 & 5.00 & 5.00 \\
\hline$C B B D C$ & 0.145 & 99.855 & 13.15 & 262.67 & 4.97 & 5.00 & 5.04 \\
\hline$C B B D C$ a & 0.024 & 99.976 & 13.13 & 262.73 & 4.98 & 5.00 & 5.01 \\
\hline$C B B D C \mathrm{~b}$ & 0.119 & 99.881 & 13.14 & 262.66 & 4.97 & 5.00 & 5.04 \\
\hline$C B B D C \mathrm{ab}$ & 0.00004 & 100.00 & 13.13 & 262.71 & 5.00 & 5.00 & 5.00 \\
\hline$C B B D C \mathrm{c}$ & 0.0002 & 100.00 & 13.13 & 262.71 & 5.00 & 5.00 & 5.00 \\
\hline$T V B D$ & 0.032 & 99.968 & 13.13 & 262.71 & 4.98 & 5.00 & 5.02 \\
\hline$T V B D$ a & 0.009 & 99.991 & 13.13 & 262.74 & 4.99 & 5.00 & 5.00 \\
\hline$T V B D \mathrm{~b}$ & 0.022 & 99.978 & 13.13 & 262.71 & 5.00 & 5.00 & 5.00 \\
\hline$T V B D \mathrm{ab}$ & 0.00001 & 100.00 & 13.13 & 262.71 & 5.00 & 5.00 & 5.00 \\
\hline$T V B D \mathrm{c}$ & 0.0002 & 100.00 & 13.13 & 262.71 & 5.00 & 5.00 & 5.00 \\
\hline TVmod & 0.049 & 99.951 & 13.13 & 262.71 & 4.98 & 5.00 & 5.02 \\
\hline TVmod a & 0.014 & 99.986 & 13.13 & 262.72 & 4.99 & 5.00 & 5.01 \\
\hline$T V \bmod \mathrm{b}$ & 0.036 & 99.964 & 13.13 & 262.70 & 4.98 & 5.00 & 5.02 \\
\hline TVmod ab & 0.00001 & 100.00 & 13.13 & 262.71 & 5.00 & 5.00 & 5.00 \\
\hline TVmod c & 0.0002 & 100.00 & 13.13 & 262.71 & 5.00 & 5.00 & 5.00 \\
\hline$T V B D C$ & 0.053 & 99.947 & 13.13 & 262.71 & 4.98 & 5.00 & 5.02 \\
\hline$T V B D C$ a & 0.014 & 99.986 & 13.13 & 262.74 & 4.99 & 5.00 & 5.01 \\
\hline$T V B D C \mathrm{~b}$ & 0.039 & 99.961 & 13.13 & 262.71 & 4.98 & 5.00 & 5.02 \\
\hline$T V B D C \mathrm{ab}$ & 0.00001 & 100.00 & 13.13 & 262.71 & 5.00 & 5.00 & 5.00 \\
\hline$T V B D C \mathrm{c}$ & 0.0002 & 100.00 & 13.13 & 262.71 & 5.00 & 5.00 & 5.00 \\
\hline $\operatorname{Exp}$ & 0.926 & 99.074 & 13.24 & 262.67 & 4.95 & 5.04 & 5.14 \\
\hline Exp a & 0.247 & 99.753 & 13.15 & 263.08 & 4.95 & 5.00 & 5.05 \\
\hline Exp b & 0.690 & 99.310 & 13.22 & 262.32 & 4.96 & 5.04 & 5.12 \\
\hline $\operatorname{Exp} a b$ & 0.006 & 99.994 & 13.13 & 262.71 & 4.99 & 5.00 & 5.01 \\
\hline $\operatorname{Exp} \mathrm{c}$ & 0.0002 & 100.00 & 13.13 & 262.71 & 5.00 & 5.00 & 5.00 \\
\hline Nexp & 0.951 & 99.049 & 13.24 & 262.63 & 4.95 & 5.04 & 5.14 \\
\hline$N \exp$ a & 0.254 & 99.746 & 13.15 & 263.07 & 4.95 & 5.00 & 5.05 \\
\hline$N \exp b$ & 0.694 & 99.306 & 13.21 & 262.32 & 4.95 & 5.04 & 5.12 \\
\hline$N \exp a b$ & 0.006 & 99.994 & 13.13 & 262.71 & 4.99 & 5.00 & 5.01 \\
\hline$N \exp \mathrm{c}$ & 0.0002 & 100.00 & 13.13 & 262.71 & 5.00 & 5.00 & 5.00 \\
\hline
\end{tabular}

$W=$ AGB estimation, $R S E=$ relative standard error, $L L=$ lower limit of confidence interval, $U L=$ upper limit of confidence interval. Datasets from which measurement errors were calculated: $C B=C$ aliper+Blume-Leiss dataset, $T V=T a p e+V e r t e x$ dataset, Exp=students with experience, $N$ exp=students without experience. Scenario used to obtain measurement error parameters: $\bmod =$ linear regression model, $B D=$ best-fitted distribution, $B D C=$ best-fitted distribution by class. Source of uncertainty: $\mathrm{a}=D B H, \mathrm{~b}=T H, \mathrm{ab}=\mathrm{correlation}, \mathrm{c}=$ prediction model.

Table VIII-41. Result of Monte-Carlo simulation Method on the contribution by uncertainty source to the total uncertainty in AGB estimation in Göttingen, Germany. 

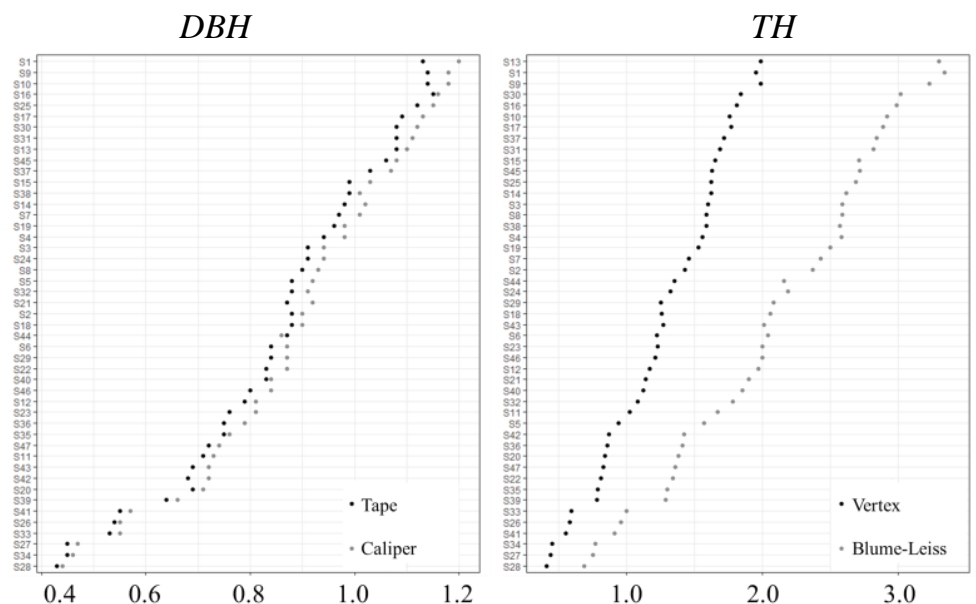

\author{
Scenario
Best Probability Density Function fitted to the measurement errors $(B P D F)$
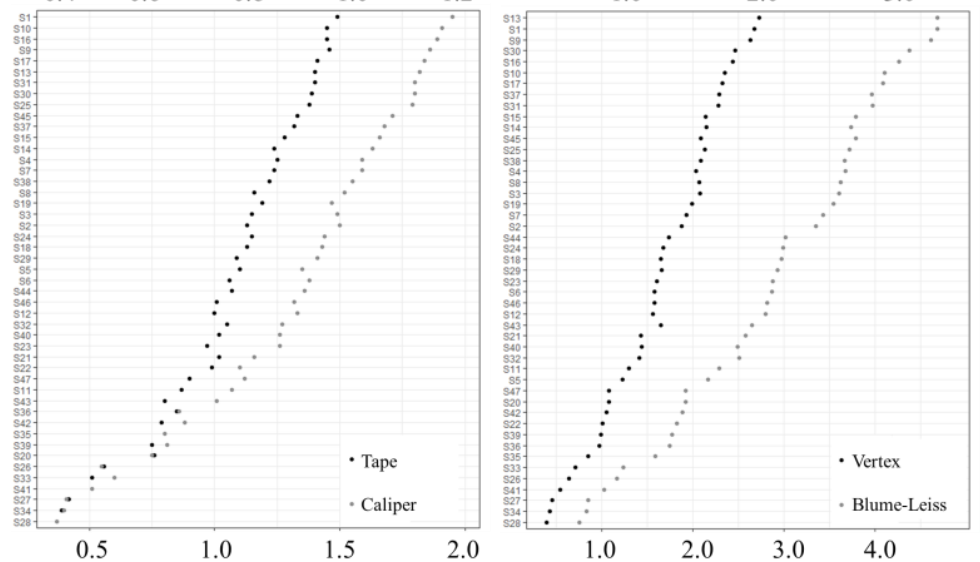

Best Probability Density Function fitted by class to the measurement errors

$(B D C)$

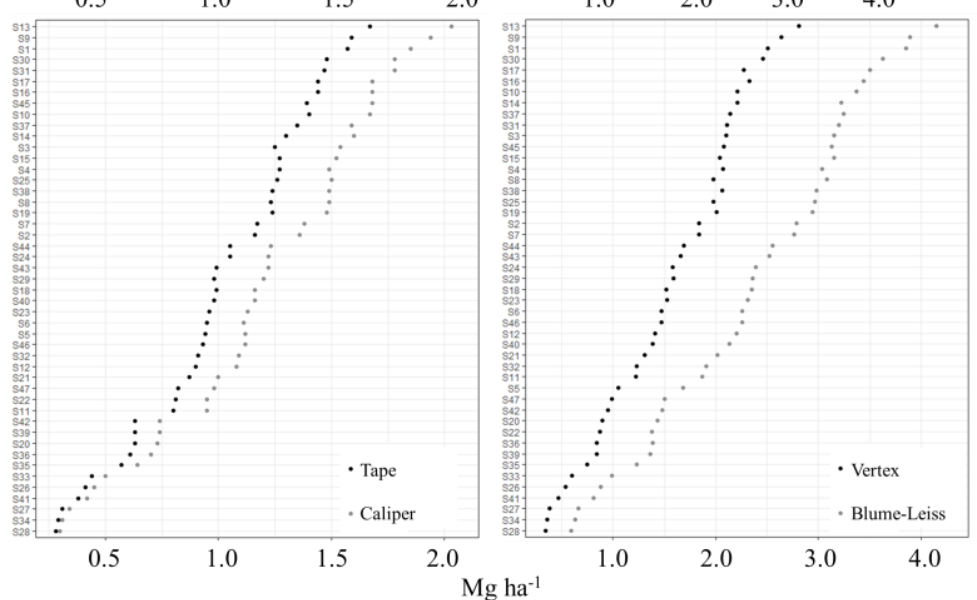

Linear model fitted to estimate the measurement uncertainty according to the tree size (mod)

Measurement error: $C=$ Caliper, $T=$ Tape, $B L=$ Blume-Leiss, V=Vertex. Scenario used to obtain measurement error parameters: $m o d=l i n e a r$ regression model, $B P D F=$ best-ftted distribution, $B D C=$ best-fitted distribution by class.

Figure VIII-2. Comparison of uncertainty estimation (paired plot) per plot and per measuring device for each scenario used to estimate measurement error parameters. 


\section{VIII.6 Appendix VI}

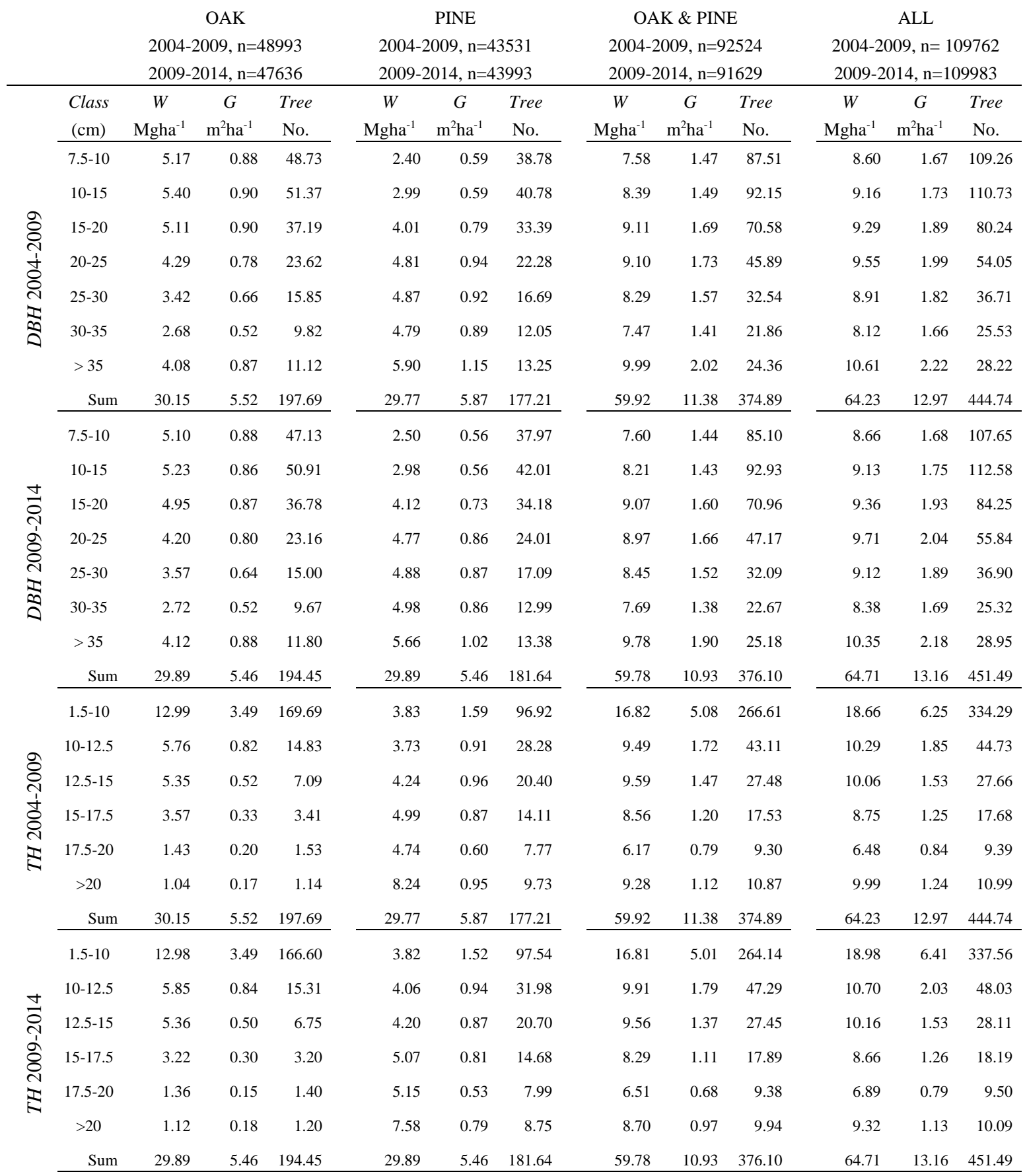

ALL=estimation made with all the data of temperate forest, PINE=estimation made with genus Pinus spp, OAK=estimation made with genus Quercus spp, $\mathrm{P} \& \mathrm{O}=$ estimation made with Pinus spp + Quercus spp.

Table VIII-42. Basal area $(G)$ and AGB $(W)$ estimation made by classes of $D B H$ and $T H$, in four datasets of Durango temperate forest. Estimation made with data of two periods of MNFI 20042009 and 2009-2014. 


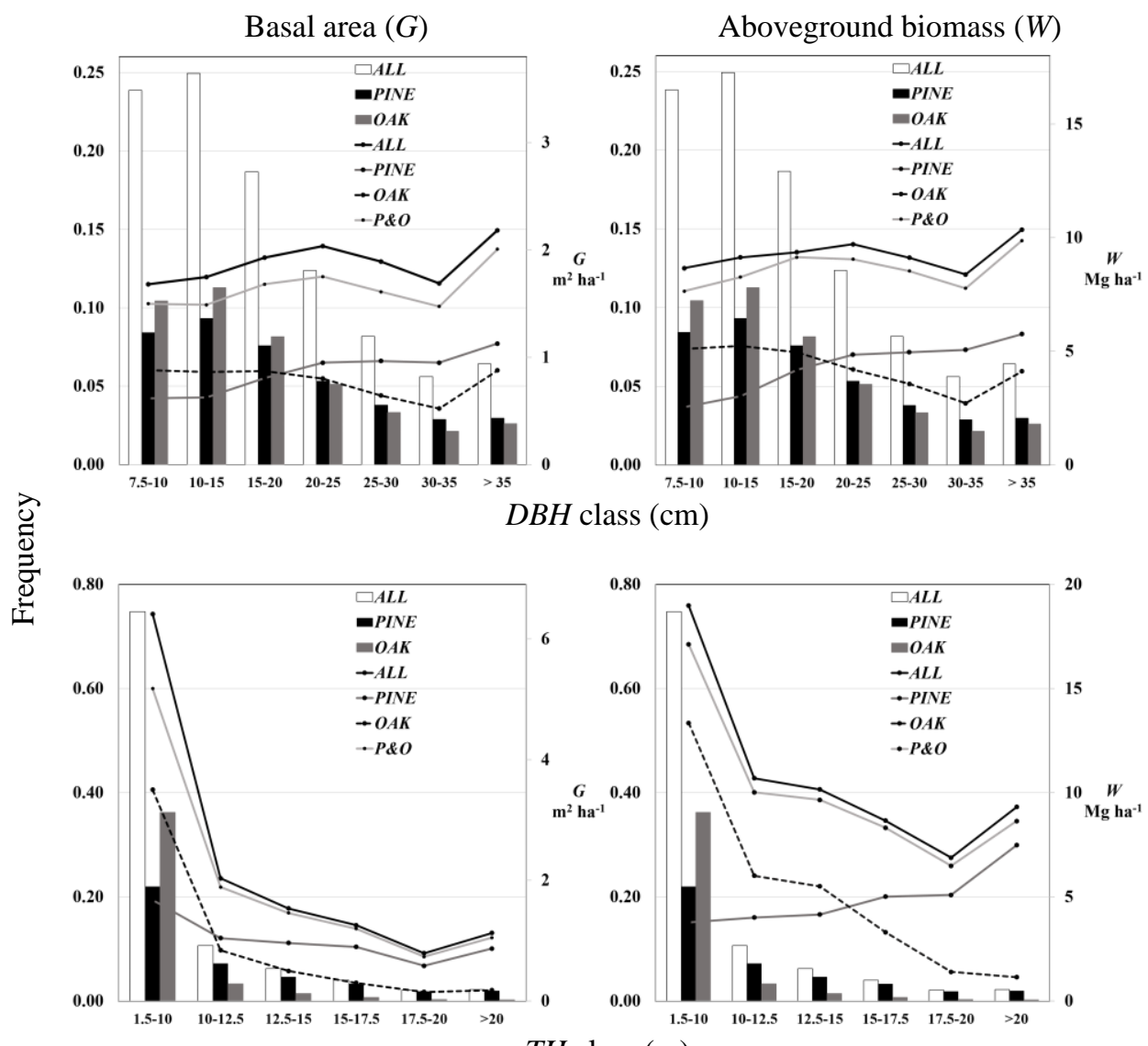

TH class (m)

ALL=estimation made with all the data of temperate forest, PINE=estimation made with genus Pinus spp ( $\mathrm{n}=43993)$, OAK=estimation made with genus Quercus spp ( $\mathrm{n}=47636), \mathrm{P} \& \mathrm{O}=$ estimation made with Pinus spp + Quercus spp $(\mathrm{n}=91629)$.

Figure VIII-3. Basal area $(G)$ and AGB $(W)$ estimation for MNFI 2009-2014 in Durango, Mexico. Above, estimations made by $D B H$ class; below, estimations made by $T H$ class. 


\section{VIII.7 Appendix VII}

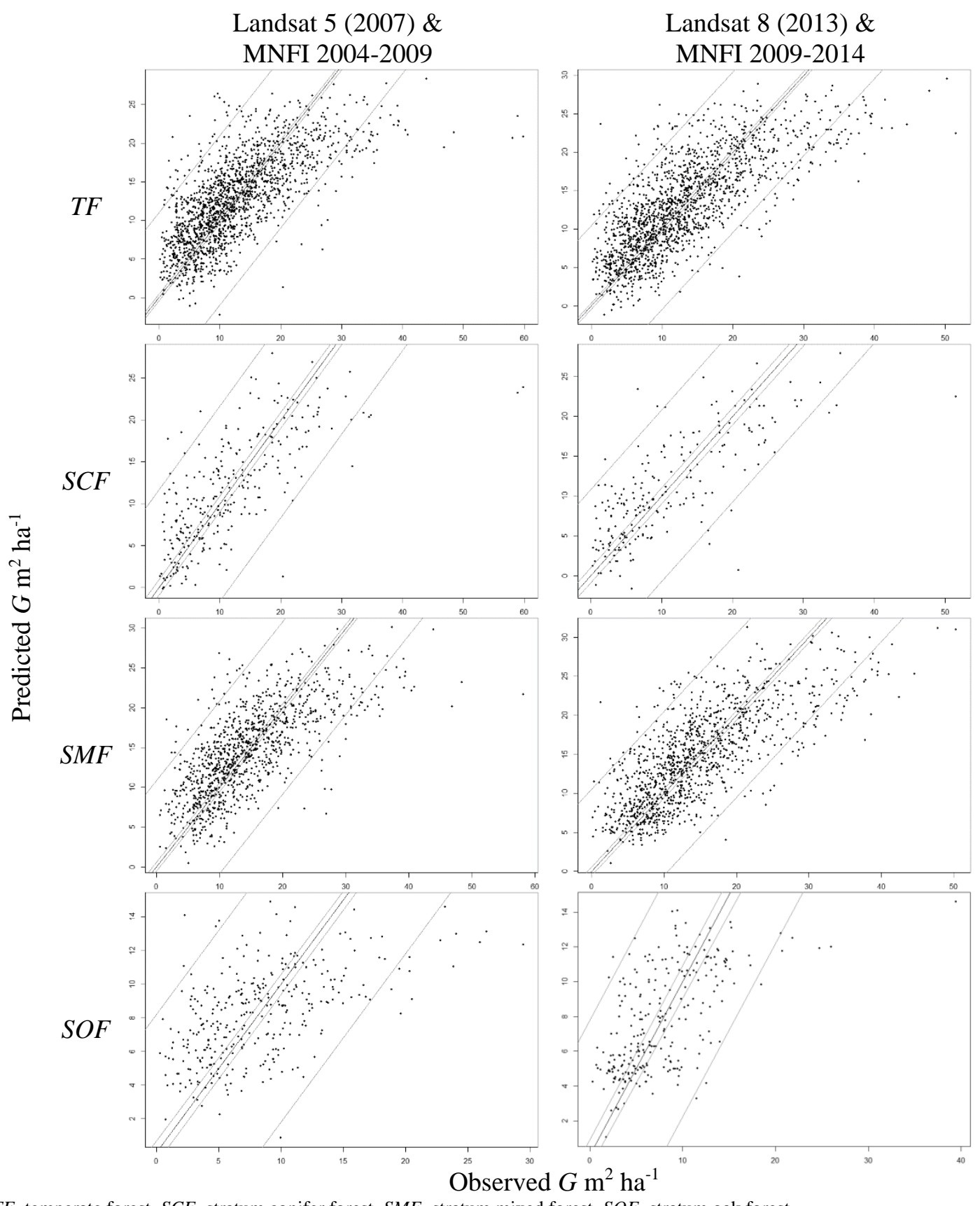

$T F=$ temperate forest, $S C F=$ stratum conifer forest, $S M F=$ stratum mixed forest, $S O F=$ stratum oak forest

Figure VIII-4. Predicted vs. observed in AGB estimate $(G)$ when applying Landsat-adjusted models and MNFI information in Durango, Mexico. 


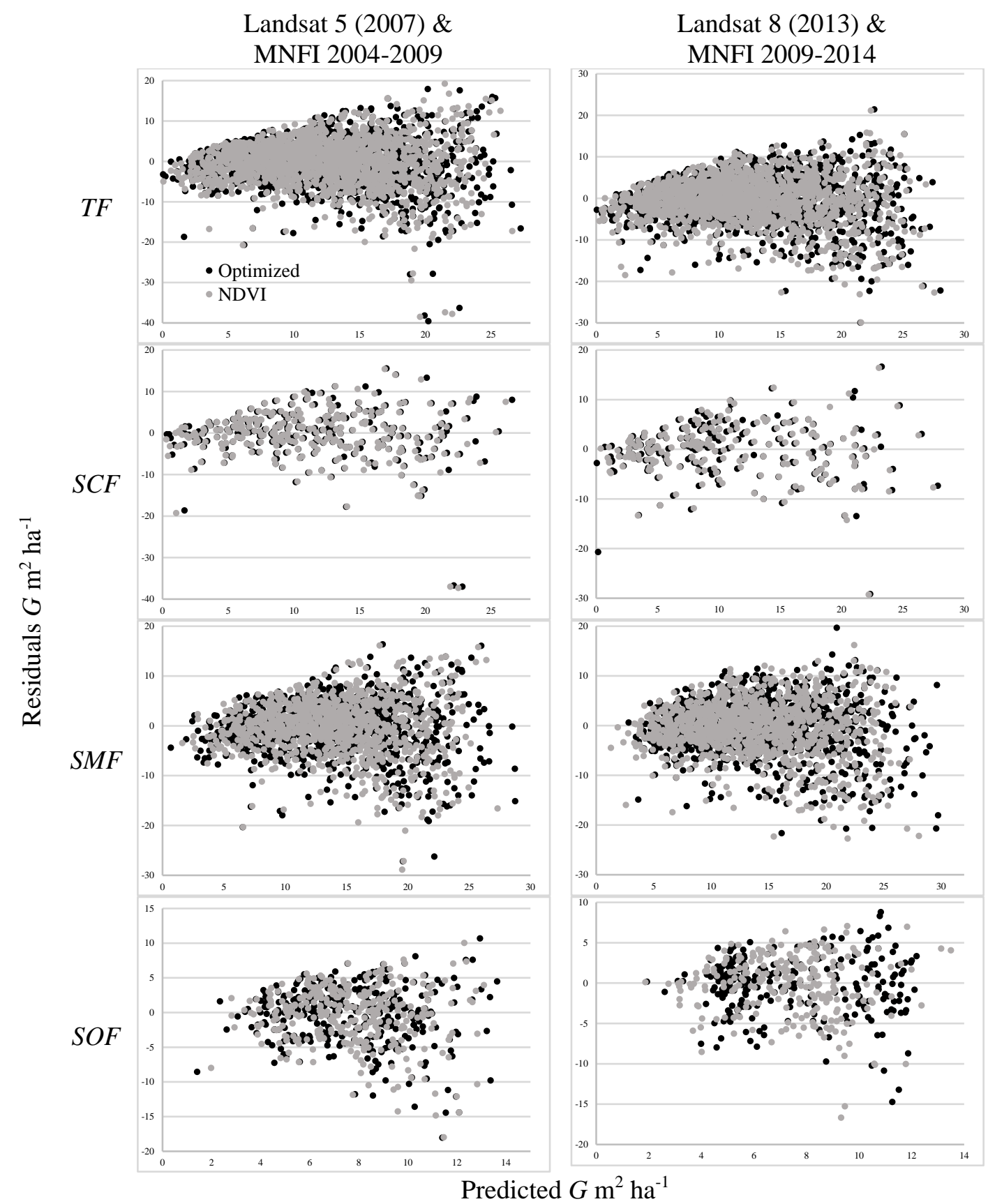

$T F=$ temperate forest, $S C F=$ stratum conifer forest, $S M F=$ stratum mixed forest, $S O F=$ stratum oak forest

Figure VIII-5. Residual vs. predicted graphs in basal area estimation $(G)$ applying linear regression models with Landsat and MNFI data. 


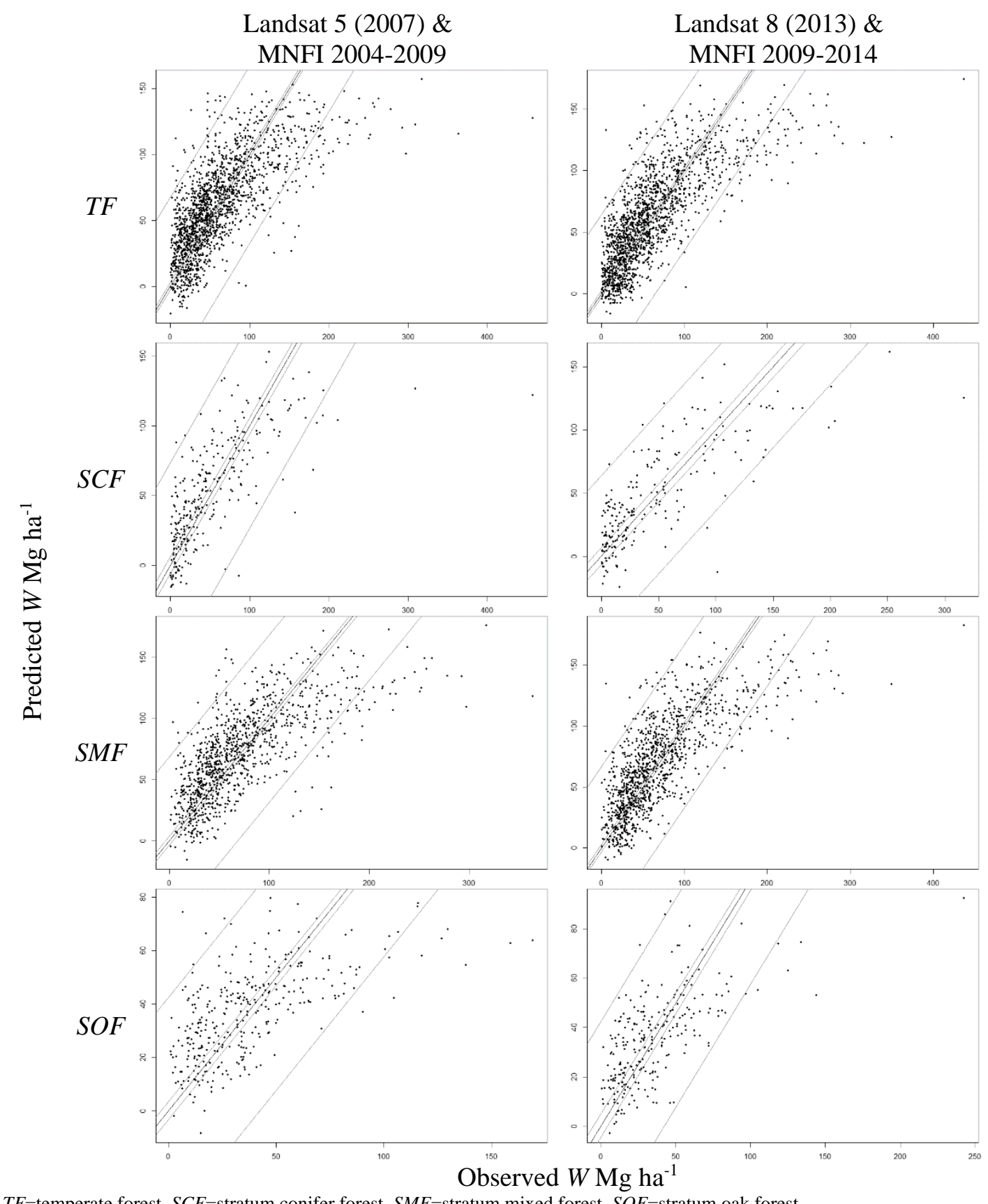

$T F=$ temperate forest, $S C F=$ =stratum conifer forest, $S M F=$ stratum mixed forest, $S O F=$ stratum oak forest

Figure VIII-6. Predicted vs. observed in AGB estimate $(W)$ when applying Landsat-adjusted models and MNFI information in Durango, Mexico. 


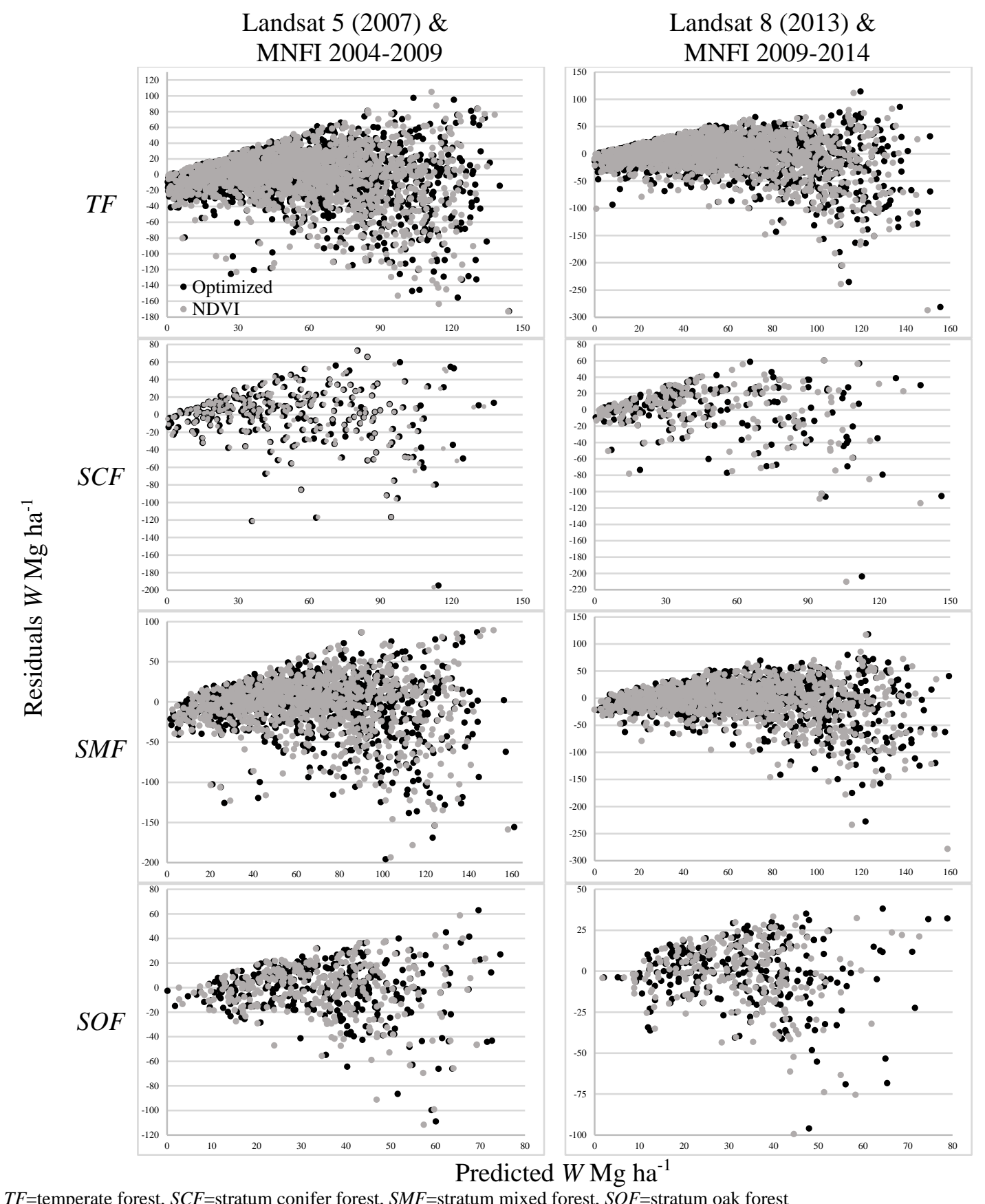

Figure VIII-7. Residual vs. predicted graphs in AGB estimation $(W)$ applying linear regression models with Landsat and MNFI data. 


\section{VIII.8 Appendix VIII}

\begin{tabular}{|c|c|c|c|}
\hline & $\begin{array}{c}G \text { class } \\
\left(\mathrm{m}^{2} \mathrm{ha}^{-1}\right)\end{array}$ & $\begin{array}{l}\text { Area } \\
\text { (ha) }\end{array}$ & $\begin{array}{c}\% \\
\text { surface }\end{array}$ \\
\hline \multirow{8}{*}{$\begin{array}{l}\text { Landsat } 5 \text { imagery } \\
\text { (2007) \& MNFI } \\
2004-2009\end{array}$} & {$[0-5)$} & 338047.11 & 6.56 \\
\hline & {$[5-10)$} & 1532279.61 & 29.74 \\
\hline & {$[10-15)$} & 1569250.44 & 30.46 \\
\hline & {$[15-20)$} & 1124598.24 & 21.83 \\
\hline & {$[20-25)$} & 517285.35 & 10.04 \\
\hline & {$[25-30)$} & 69564.78 & 1.35 \\
\hline & [30-35] & 1147.23 & 0.02 \\
\hline & Total & 5152172.76 & 100.00 \\
\hline \multirow{8}{*}{$\begin{array}{c}\text { Landsat } 8 \text { imagery } \\
\text { (2013) \& MNFI } \\
\text { 2009-2014 }\end{array}$} & {$[0-5)$} & 340221.96 & 6.67 \\
\hline & {$[5-10)$} & 1647123.66 & 32.27 \\
\hline & {$[10-15)$} & 1467709.29 & 28.76 \\
\hline & {$[15-20)$} & 992527.56 & 19.45 \\
\hline & {$[20-25)$} & 549831.24 & 10.77 \\
\hline & {$[25-30)$} & 105864.84 & 2.07 \\
\hline & [30-35] & 740.16 & 0.01 \\
\hline & Total & 5104018.71 & 100.00 \\
\hline
\end{tabular}

Table VIII-43. Temperate forest surface by basal area $(G)$ class in Durango, Mexico.

\begin{tabular}{|c|c|c|c|c|c|}
\hline & $\begin{array}{l}W \text { class } \\
\text { Mg ha }^{-1}\end{array}$ & $\begin{array}{c}\text { Area } \\
\text { (ha) }\end{array}$ & $\begin{array}{c}\% \\
\text { surface }\end{array}$ & $\begin{array}{c}W \\
(\mathrm{Tg})\end{array}$ & $\begin{array}{c}\% \\
\text { AGB } \\
\end{array}$ \\
\hline \multirow{5}{*}{$\begin{array}{c}\text { Landsat } 5 \text { imagery } \\
\text { (2007) \& MNFI } \\
\text { 2004-2009 }\end{array}$} & {$[0-50)$} & 2443025.70 & 47.42 & 59.95 & 21.01 \\
\hline & {$[50-100)$} & 2190043.44 & 42.51 & 161.23 & 56.52 \\
\hline & {$[100-150)$} & 511730.46 & 9.93 & 62.79 & 22.01 \\
\hline & [150 - 205] & 7373.16 & 0.14 & 1.27 & 0.44 \\
\hline & Total & 5152172.76 & 100.00 & 285.25 & 100.00 \\
\hline \multirow{5}{*}{$\begin{array}{c}\text { Landsat } 8 \text { imagery } \\
\text { (2013) \& MNFI } \\
\text { 2009-2014 }\end{array}$} & {$[0-50)$} & 2650856.04 & 51.93 & 65.39 & 23.92 \\
\hline & {$[50-100)$} & 1925092.89 & 37.72 & 142.50 & 52.12 \\
\hline & {$[100-150)$} & 520412.94 & 10.20 & 64.21 & 23.48 \\
\hline & [150 - 205] & 7656.84 & 0.15 & 1.32 & 0.48 \\
\hline & Total & 5104018.71 & 100.00 & 273.42 & 100.00 \\
\hline
\end{tabular}

Table VIII-44. Temperate forest surface by AGB $(W)$ class and amount of $W$ stored by class in Durango, Mexico. 